VRIJE

UNIVERSITEIT

BRUSSEL

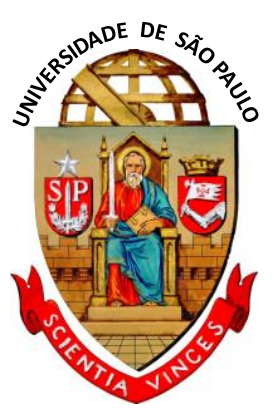

\title{
MODELLING AND ANALYSIS OF HYPERBOLIC METAMATERIALS FOR CONTROLLING THE SPONTANEOUS EMISSION RATE AND EFFICIENCY OF QUANTUM EMITTERS
}

Doctoral thesis submitted in fulfilment of the requirements for the academic degree of Doctor in Engineering and Doctor in Science by

\section{ACHILES FONTANA DA MOTA}

February 2019

VUB Promotors:

Prof. Dr. Ir. Heidi Ottevaere

Prof. Dr. Ir. Wendy Meulebroeck
USP Promotor:

Prof. Dr. Ben-Hur V. Borges

Vrije Universiteit Brussel (VUB)

Department of Applied Physics and Photonics

Brussels Photonics Team, B-PHOT

University of São Paulo (USP)

São Carlos School of Engineering

Department of Electrical and Computer Engineering 


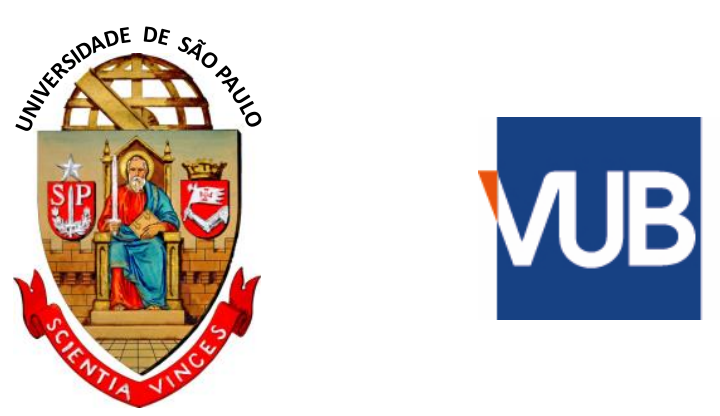

VRIJE

UNIVERSITEIT

BRUSSEL

\section{MODELO E ANÁLISES DE METAMATERIAIS HIPERBÓLICOS PARA O CONTROLE DA TAXA DE EMISSÃO ESPONTÂNEA E EFICIÊNCIA DE EMISSORES QUÂNTICOS}

Tese apresentada em cumprimento dos requisitos para o grau acadêmico de Doutor em Ciências, Programa de Engenharia Elétrica, Telecomunicações, por

\section{ACHILES FONTANA DA MOTA}

Fevereiro 2019

USP Promotor:

Prof. Dr. Ben-Hur V. Borges
VUB Promotors:

Prof. Dr. Ir. Heidi Ottevaere

Prof. Dr. Ir. Wendy Meulebroeck

Universidade de São Paulo (USP)

Escola de Engenharia de São Carlos

Departamento de Engenharia Elétrica e de Computação

Vrije Universiteit Brussel (VUB)

Department of Applied Physics and Photonics

Brussels Photonics Team, B-PHOT

Trata-se da versão corrigida da tese. A vesão original se encontra disponível na EESC/USP que aloja o Programa de Pós-Graduação de Engenharia Elétrica. 
AUTORIZO A REPRODUÇÃO TOTAL OU PARCIAL DESTE TRABALHO, POR QUALQUER MEIO CONVENCIONAL OU ELETRÔNICO, PARA FINS DE ESTUDO E PESQUISA, DESDE QUE CITADA A FONTE.

Ficha catalográfica elaborada pela Biblioteca Prof. Dr. Sérgio Rodrigues Fontes da EESC/USP com os dados inseridos pelo(a) autor(a).

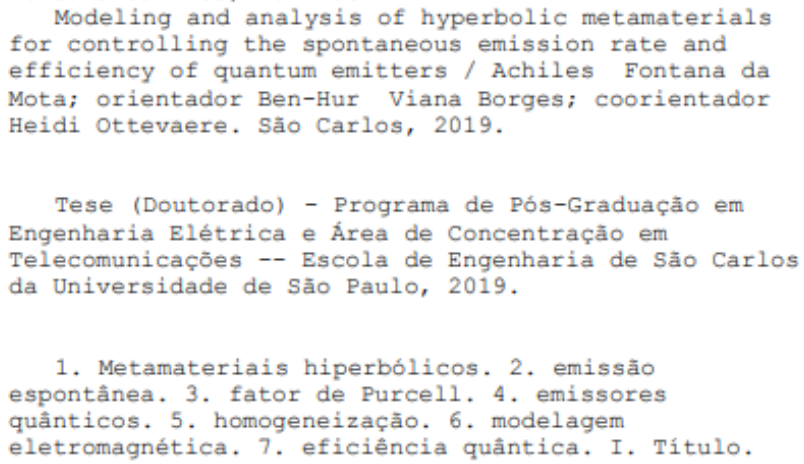

Eduardo Graziosi Silva - CRB - 8/8907 


\section{Members of the Jury}

Prof. Dr. Ben-Hur Viana Borges, promotor Department of Electrical and Computer Engineering

University of São Paulo, Brazil

Prof. Dr. Ir. Heidi Ottevaere, promotor Department of Applied Physics and Photonics

Vrije Universiteit Brussel, Belgium

Prof. Dr. Ir. Wendy Meulebroeck, promotor Department of Applied Physics and Photonics

Vrije Universiteit Brussel, Belgium

Prof. Dr. Ir. Peter Schelkens, chair

Department of Electronics and Informatics

Vrije Universiteit Brussel, Belgium

Prof. Dr. Roger Vounckx, vice-chair Department of Electronics and Informatics

Vrije Universiteit Brussel, Belgium

Prof. Dr. Ir. Hugo Thienpont, secretary Department of Applied Physics and Photonics

Vrije Universiteit Brussel, Belgium

Prof. Dr. Philippe Wilhelm Courteille

São Carlos Institute of Physics University of São Paulo, Brazil

Prof. Dr. Thiago Pedro Mayer Alegre "Gleb Wataghin" Institute of Physics University of Campinas, Brazil

Prof. Dr. Sci. Andrei Lavrinenko

Department of Photonics Engineering Technical University of Denmark, Denmark

Prof. Dr. Gustavo Silva Wiederhecker

"Gleb Wataghin" Institute of Physics

University of Campinas, Brazil 



\section{Errata}

MOTA, A. F. Modeling and analysis of hyperbolic metamaterials for controlling the spontaneous emission rate and efficiency of quantum emitters. Thesis (Ph. D.), São Carlos School of Engineering, University of São Paulo, São Carlos, 2019.

Text changes:

\begin{tabular}{|c|c|c|c|}
\hline Page & Line & Where it read & Now it reads \\
\hline 12 & 8 & $\begin{array}{l}\text {..In this dissertation, } \\
\text { QEs are considered } \\
\text { infinitesimal dipoles } \\
\text { governed by the classical } \\
\text { electromagnetic } \\
\text { equations [109]. The... }\end{array}$ & $\begin{array}{l}\text {... In this dissertation, we assume that the QEs are } \\
\text { under weak coupling regime and low excitation } \\
\text { power to avoid saturation [109]. Under these } \\
\text { assumptions, the QEs can be modelled as infinitesimal } \\
\text { dipoles governed by classical electromagnetic } \\
\text { equations. The... }\end{array}$ \\
\hline 13 & 17 & $\begin{array}{l}\text {...mechanisms to reduce } \\
\tau \text { o. One... }\end{array}$ & $\begin{array}{l}\text {...mechanisms to reduce } \tau 0 \text {. Moreover, enhancing the } \\
\text { modulation speed by decreasing the QEs' lifetime is } \\
\text { crucial for light emitting diode (LED) and laser } \\
\text { technologies. One... }\end{array}$ \\
\hline 13 & 22 & $\begin{array}{l}\text {...decaying to } \mathrm{S}_{0}\left(\tau_{s}\right) \text {. } \\
\text { Consequently, the QE } \\
\text { lifetime in the proposed } \\
\text { situation }\left(\tau_{s}\right) \text { is reduced } \\
\left(\tau_{s}<\tau 0\right) \text { while the } \\
\text { spontaneous emission } \\
\left(\Gamma_{s}\right) \text { rate is enhanced } \\
\left(\Gamma_{s}>\Gamma_{0}\right) \ldots\end{array}$ & $\begin{array}{l}\text {... decaying to } S_{0}\left(\tau_{s}\right) \text {. The Fermi's golden rule can be } \\
\text { written as [38],[110], } \\
\qquad \Gamma_{s}=\frac{1}{\tau_{s}}=\frac{2 \pi}{h}\left|\left\langle S_{1}\left|H_{\text {int }}(k, \sigma)\right| S_{0}\right\rangle\right|^{2} \rho_{s}\left(E_{f}\right) \text {, } \\
\text { where } \rho_{s}\left(E_{f}\right) \text { is the density of states (DES) at the } \\
\text { energy } E_{f},\left\langle S_{0}\left|H_{i n t}(k, \sigma)\right| S_{1}\right\rangle \text { is the matrix element of } \\
\text { the transition from the initial state } \mid S_{0}>\text { to the final } \\
\text { state }\left|S_{1}\right\rangle \text { with wavenumber } k \text { and polarization } \sigma \text {. } \\
\text { Since } \rho_{s}\left(E_{f}\right) \text { can be written as a summation of all } \\
\text { possible decay modes [38], i.e., } \\
\qquad \rho_{s}\left(E_{f}\right)=\sum_{i=1, \ldots N} \delta\left(c k_{i}-\omega\right) \text {, } \\
\text { enhancing the number of possible decay modes } \\
\text { increases the possibility of the electron to decay, thus } \\
\text { enhancing } \Gamma_{s} \text {. Moreover, the higher the number of } \\
\text { decay modes, the shorter the QE's lifetime }\left(\tau_{s}<\tau\right) \text {.... }\end{array}$ \\
\hline 17 & 20 & $\begin{array}{l}\text {...high quantum } \\
\text { efficiency using... }\end{array}$ & $\begin{array}{l}\text {...high external quantum efficiency (for the sake of } \\
\text { simplicity, from hereon the term "external" is omitted } \\
\text { when related to the quantum efficiency) using... }\end{array}$ \\
\hline 26 & 8 & $\begin{array}{l}\ldots \text { sake of simplicity. } \\
\text { Since... }\end{array}$ & $\begin{array}{l}\text {... sake of simplicity. Moreover, note that we use bold } \\
\text { and italic non-bold notation to represent vectors and } \\
\text { their magnitude, respectively (e.g. vector A has } \\
\text { magnitude } A \text { ). Since... }\end{array}$ \\
\hline 48 & $\begin{array}{l}\text { Fig. } 3.1 \\
\text { caption }\end{array}$ & $\begin{array}{l}\text { Absorption (red) and } \\
\text { emission (blue) of the } \\
\text { Cy7 }\end{array}$ & $\begin{array}{l}\text { Absorption (red) and emission (blue) of Cy7. Data } \\
\text { obtained from the Cy7 datasheet. }\end{array}$ \\
\hline
\end{tabular}




\begin{tabular}{|c|c|c|c|}
\hline 60 & 7 & ... maintaining a high $P_{\text {eff. }}$ & $\begin{array}{l}\text {... maintaining a high } P_{\text {eff. }} \\
\text { Thinking in corroborating the approach proposed in } \\
\text { chapter } 3 \text { of this thesis, the PhD candidate spent the } \\
\text { year of } 2017 \text { at the VUB under the supervision of Profs. } \\
\text { Heidi Ottevaere and Wendy Meulebroeck. During this } \\
\text { period, we have fully characterized the emission } \\
\text { spectrum and the lifetime of Cy7. Moreover, we also } \\
\text { have characterized the process to mix the PMMA layer } \\
\text { with dichloromethane to control the thickness of the } \\
\text { PMMA layer. Later, we have hired a foundry to } \\
\text { fabricate the HMM proposed on Figure } 3.2 \text {, but during } \\
\text { the characterization of the structure we found out that } \\
\text { the fabrication process had not been performed as } \\
\text { expected. Consequently, we could not perform the } \\
\text { Cy7 lifetime measurements on the top of the HMM. } \\
\text { This characterization and lifetime measurements are } \\
\text { described in Appendix B. }\end{array}$ \\
\hline 96 & 11 & $\begin{array}{l}\ldots \text { the desired } \Gamma_{\text {eff. }} \text { The } \\
\text { optimization... }\end{array}$ & $\begin{array}{l}\text {...the desired } \Gamma_{\text {eff. Differently from previous }} \\
\text { approaches based on single QE at a fixed position } \\
{[111] \text {, the proposed optimization procedure takes the }} \\
\text { behavior of all QEs embedded in the system into } \\
\text { account. The optimization... }\end{array}$ \\
\hline 96 & 29 & ... to FDTD simulations. & $\begin{array}{l}\text {... to FDTD simulations. All the MATLAB codes used } \\
\text { to solve the scenarios proposed in this chapter are } \\
\text { available in [i]. }\end{array}$ \\
\hline 97 & $\begin{array}{l}\text { Fig. } 5.1 \\
\text { caption }\end{array}$ & $\begin{array}{l}\text {.., creating two } \\
\text { stationary fields }(\mathbf{A} \ldots\end{array}$ & $\ldots$, creating two electric fields (A ... \\
\hline 98 & 5 & 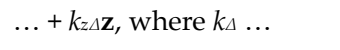 & 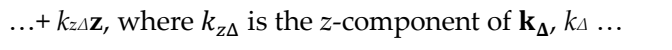 \\
\hline 98 & 11 & $\begin{array}{l}\ldots \bar{\Delta}=S \text { or } \mathrm{C}, \\
\text { respectively. } \\
\text { Furthermore, } \ldots\end{array}$ & $\begin{array}{l}\ldots \bar{\Delta}=S \text { or } \mathrm{C} \text {, respectively, } a \text { and } b \text { are the distances } \\
\text { from source } \mathrm{L} \text { to the layer } w-1 \text { and } w+1 \text {, respectively } \\
\left(a+b=h \text {, where } h \text { is } w^{\text {th }} \text { layer thickness). Furthermore, } \ldots\right.\end{array}$ \\
\hline 98 & 13 & 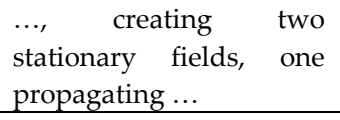 & $\ldots$, creating two electric fields, one propagating ... \\
\hline 101 & 9 & Finally, ... & $\begin{array}{l}\text { where } \theta \text { and } \phi \text { are the elevation and azimuthal angles, } \\
\theta \text { and } \varphi \text { are unitary vectors in spherical coordinates } \\
\text { along } \theta \text { and } \varphi \text { directions, respectively. Finally, ... }\end{array}$ \\
\hline 102 & 10 & The stationary fields... & The electric fields... \\
\hline 104 & 25 & ...wth layer. & $\begin{array}{l}\text { wth layer. This scenario is used to demonstrate the } \\
\text { optimization procedure. }\end{array}$ \\
\hline 105 & 3 & ...FDTD simulation. & ...FDTD simulation, as described in section 3.1 . \\
\hline 105 & 8 & $\begin{array}{l}\ldots \text { over } V_{L} \text { (defined as the } \\
\text { region }\left[-\Lambda_{x} / 2,-\Lambda_{y} / 2,-\right. \\
\text { a }] \leq[x, y, z]<\left[-\Lambda_{x} / 2,-\Lambda_{y} / 2,\right. \\
b]) . \text { is reduced to a single } \\
\text { integral over } V_{L} \text { (defined } \\
\text { as the region }\left[-\Lambda_{x} / 2,-\right. \\
\left.\Lambda_{y} / 2,-a\right] \leq[x, y, z]<\left[\Lambda_{x} / 2,\right. \\
\left.\left.\Lambda_{y} / 2, b\right]\right) . \text { Furthermore }, \ldots\end{array}$ & $\begin{array}{l}\ldots \text { over } V_{L} \text { (defined as the region }[|x|,|y|, \mid z+(a- \\
\left.b) / 2 \mid] \leq\left[\Lambda_{x} / 2, \Lambda_{y} / 2,(a+b) / 2\right]\right) \text {. Furthermore, } \ldots\end{array}$ \\
\hline
\end{tabular}




\begin{tabular}{|c|c|c|c|}
\hline 105 & $\begin{array}{l}\text { after } \\
\text { eq. } 5.36\end{array}$ & --- & $\begin{array}{l}\text { Typically, the polymers embedded with QEs are spin- } \\
\text { coated on top of the structure [2,26-29]. Consequently, } \\
\text { the QEs become uniformly distributed and oriented } \\
\text { inside the structure, as adopted in this scenario. This } \\
\text { assumption is also used in the optimization procedure } \\
\text { described in section } 3.2 \text {. }\end{array}$ \\
\hline 106 & 7 & --- & $\begin{array}{l}\text { This scenario can be used to calculate both the decay } \\
\text { curve and the external quantum efficiency when a } \\
\text { polymer embedded with QEs is spin-coated in more } \\
\text { than one layer of the nano-patterned structure. }\end{array}$ \\
\hline 108 & 10 & $\begin{array}{l}\text {...both the 3D-SAM and } \\
\text { Lumerical FDTD [87]. }\end{array}$ & $\begin{array}{l}\text {...both the 3D-SAM (using the special case shown in } \\
\text { section 5.2.2.2) and Lumerical FDTD [87]. }\end{array}$ \\
\hline 108 & 12 & ... FDTD (symbols). & $\begin{array}{l}\text { FDTD (symbols) when both sources (QEL and QEc) are } \\
\text { present. }\end{array}$ \\
\hline 108 & 19 & $\begin{array}{l}\text {...very small and } \\
\text { independent of } b_{c}, \ldots\end{array}$ & $\ldots$ very small and slightly dependent on $b_{c}, \ldots$ \\
\hline 108 & 26 & $\begin{array}{l}\ldots \text { as function of } b_{c} \text { with } \\
\text { (solid line) and } \\
\text { without... }\end{array}$ & $\begin{array}{l}\text {... as function of } b_{c} \text { with both sources present, QEL and } \\
\mathrm{QEC} \text {, (solid line) and without... }\end{array}$ \\
\hline 108 & $\begin{array}{l}\text { Fig. } 5.3 \\
\text { caption }\end{array}$ & $\begin{array}{l}\text { (a) } \Gamma_{L} / \Gamma_{0} \text { and } \Gamma_{\mathrm{C}} / \Gamma_{0} \text { using } \\
\text { 3D-SAM (red and blue } \\
\text { solid lines) and FDTD } \\
\text { (symbols). This figure } \\
\text { also shows } \Gamma_{\mathrm{C}} / \Gamma_{0} \text { obtained } \\
\text { with 3D-SAM when QEL } \\
\text { is not present (dashed } \\
\text { lines). (b) Qc/Wo with } \\
\text { (solid line) and without } \\
\text { (dashed line) QEL. }\end{array}$ & $\begin{array}{l}\text { (a) } \Gamma_{\mathrm{L}} / \Gamma_{0} \text { and } \Gamma_{\mathrm{C}} / \Gamma_{0} \text { calculated using 3D-SAM (red and } \\
\text { blue solid lines, respectively) and FDTD (circles and } \\
\text { squares, respectively) when both QEL and } \mathrm{QEC}_{\mathrm{C}} \text { are } \\
\text { present in the system. This figure also shows } \Gamma_{\mathrm{C}} / \Gamma_{0} \\
\text { obtained with 3D-SAM when QEL is not present } \\
\text { (dashed lines). (b) } Q_{\mathrm{C}} / Q_{0} \text { calculated using 3D-SAM } \\
\text { (blue solid lines) and FDTD (circles and squares, } \\
\text { respectively) when both QEL and QEc are present in } \\
\text { the system. This figure also shows } Q_{\mathrm{C}} / \mathrm{Q}_{0} \text { obtained } \\
\text { with 3D-SAM when QEL is not present (dashed lines). }\end{array}$ \\
\hline 109 & 12 & $\begin{array}{lr}\text {...are } & \text { inversely } \\
\text { proportional). } & \text { The } \\
\text { wavelength... } & \end{array}$ & $\begin{array}{l}\text {...are inversely proportional). Furthermore, since the } \\
\text { R6G's sources are assumed uniformly distributed, } \\
\text { both } P \text { and } \eta_{\text {eff }} \text { are calculated following the steps } \\
\text { described in section } 5.2 .2 .2 \text {. The wavelength... }\end{array}$ \\
\hline 109 & 26 & $\begin{array}{l}\text {...cover layer. The } \mathrm{Ag} \\
\text { and } \mathrm{SiO}_{2} \ldots\end{array}$ & $\begin{array}{l}\text {...cover layer Furthermore, we have tested alternative } \\
\text { metallic and dielectric materials }(\mathrm{Au}, \mathrm{TiO} 2, \mathrm{Si}) \text {, but } \\
\text { only } \mathrm{Ag} \text { and } \mathrm{SiO}_{2} \text { have produces the desired } \\
\text { performance. The } \mathrm{Ag} \text { and } \mathrm{SiO}_{2} \ldots\end{array}$ \\
\hline 110 & $\begin{array}{l}\text { Fig. } 5.4 \\
\text { caption }\end{array}$ & $\ldots$ of the HMM. The (b)... & $\begin{array}{l}\ldots \text { of the HMM. The substrate and air cover layers } \\
\text { (above the PMMA) are assumed semi-infinite. The } \\
\text { (b)... }\end{array}$ \\
\hline 111 & 12 & $\begin{array}{l}\begin{array}{l}\text { Note that a } 2 \mathrm{D} \text { dipole } \\
\text { behaves }\end{array} \\
\text { source } \\
\text { equivalently to a dipole } \\
\text { line in 3D, so it is denoted } \\
\text { here as 2D-dipole. After } \\
\text { these assumptions, (5.11) } \\
\text { is rewritten as follows, }\end{array}$ & $\begin{array}{l}\text { Note that a 2D dipole source can be arbitrarily } \\
\text { oriented in the } x z \text { plane (as shown in Fig. } 5(\text { a)) and still } \\
\text { behave equivalently to a dipole line along the } y \text {-axis in } \\
\text { 3D. Therefore, we denote it here as 2D-dipole. After } \\
\text { these assumptions, (5.11) is rewritten as follows }\end{array}$ \\
\hline 111 & 14 & $\begin{array}{l}\text { In this procedure, the } 2 \mathrm{D}- \\
\text { dipole embedded in } \ldots \\
\Gamma_{x} / \Gamma_{0} \text { is higher in this case } \\
{[55] .}\end{array}$ & $\begin{array}{l}\text { Although our formalism can be applied to an } \\
\text { arbitrarily oriented 2D-dipole in the xz-plane, in the } \\
\text { optimization procedure we assume the 2D-dipole } \\
\text { oriented parallel to the HMM surface, i.e., along the } x \text { - } \\
\text { axis }(\mathbf{p}=p \mathbf{x}) \text {. This assumption is justified because } 2 / 3\end{array}$ \\
\hline
\end{tabular}


of the QEs are oriented parallel to the HMM surface $(x$ and $y$ axes) in the 3D case [37]. Moreover, we also assume the 2D-dipole at a fixed position since the QEs' position affects the amplitude and phase of the evanescent fields that interact with the NPHM surface, but not with the modes responsible for outcoupling the high- $k$ waves. These modes are affected by the nanopatterns geometrical parameters. Consequently, optimizing $\Gamma_{x} / \Gamma_{0}$ and $\eta_{x}$ at one position is enough to guarantee that both parameters are optimized for any position inside the host layer. In this sense, we assume the 2D-dipole embedded in a $40 \mathrm{~nm}$ thick PMMA host centered at the NPHM ridge at $a=10$ $n m$ from the NPHM surface because $\Gamma_{x} / \Gamma_{0}$ is higher in this case [55]

Fig. 5.5 caption (a) 2D-NHMM consisting of 9 alternating $\mathrm{Ag} / \mathrm{SiO}_{2}$ layers with period $\Lambda_{x}$ and fill factor $f_{2 D}$ on top of a $\mathrm{SiO}_{2}$ substrate. The PMMA host layer with thickness $h$ is on top of the NHMM. (b) and (c) are the $\Gamma_{x} / \Gamma_{0}$ and $\eta_{x}$ maps, respectively, as function of $\Lambda x$ and $f_{2 D}$ of a 2Ddipole placed at the center of the ridge at a distance $a=10 \mathrm{~nm}$ from the NPHM surface. The dashed black lines in (b) and (c) represent the LSPP, while the white lines represent the $f_{3 D}$ of a 3D structure with $\rho_{g}=$ $50 \mathrm{~nm}$ (a) 2D-NHMM consisting of 9 alternating $\mathrm{Ag} / \mathrm{SiO}_{2}$ layers with period $\Lambda_{x}$ and fill factor $f_{2 D}$ on top of a $\mathrm{SiO}_{2}$ substrate. The arbitrarily oriented $\mathrm{QE}$ is embedded in the PMMA host layer with thickness $h$ on top of the NHMM. (b) and (c) are the $\Gamma_{x} / \Gamma_{0}$ and $\eta_{x}$ maps, respectively, as function of $\Lambda_{x}$ and $f_{2 D}$ of a 2Ddipole placed at the center of the ridge at a distance $a=10 \mathrm{~nm}$ from the NPHM surface. Notice that the QE is assumed oriented along the $x$-axis in the calculation of $\Gamma_{x} / \Gamma_{0}$ and $\eta$. Moreover, the dashed black lines in (b) and (c) represent the LSPP, while the white lines represent $f_{3 D}$ of a 3D structure with $\rho_{8}=50 \mathrm{~nm}$. 


\section{Figure changes:}

Figure 1.7 has been replaced by:

(a)
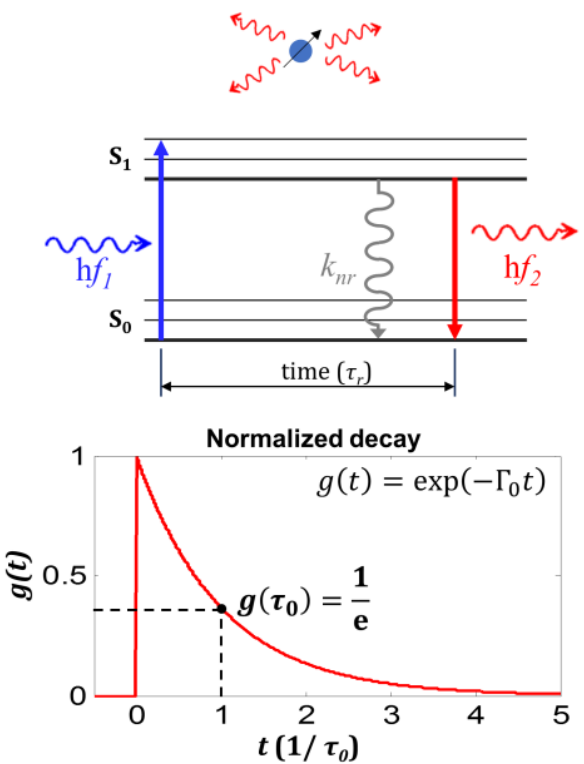

(b)
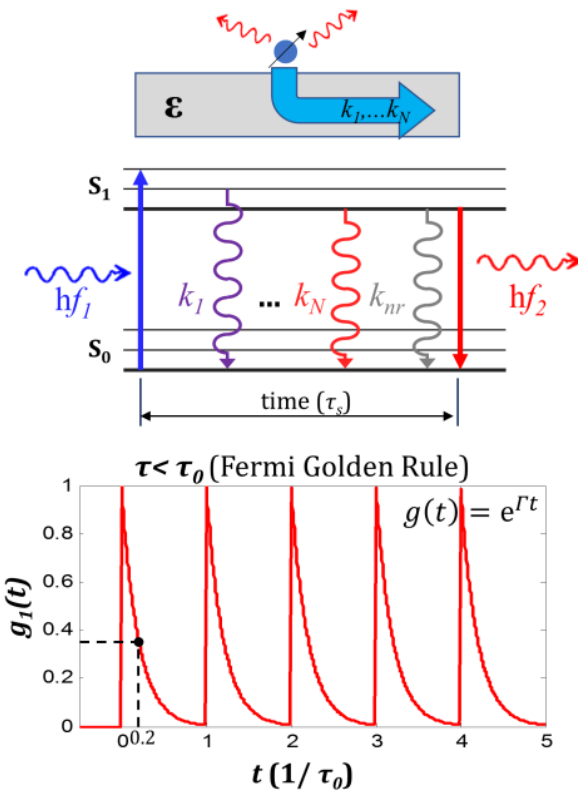

Figure 1.1. Quantum emitters (top) in (a) free space and (b) close to a structure supporting a large number of modes, and their respective energy level diagrams (middle, showing excitation and decay routes) and normalized decay curves (bottom). In (a), a photon with energy $E_{1}=h f_{1}$ excites an electron of the highest occupied molecular orbital ( $\left.\mathrm{S}_{0}\right)$ to the lowest unoccupied molecular orbital $\left(\mathrm{S}_{1}\right) .\left(k_{1}, \ldots, k_{N}, k_{n r}, h f_{2}\right)$ and $\left(k_{n r}, h f_{2}\right)$ represent the decay routes for a QE in scenario (a) and (b), respectively. $\left(\Gamma_{0}, \tau_{0}\right)$ and $(\Gamma, \tau)$ are the spontaneous emission rate and the QE lifetime in scenario (a) and (b), respectively. 
Figure 5.4 has been replaced by:

(a)

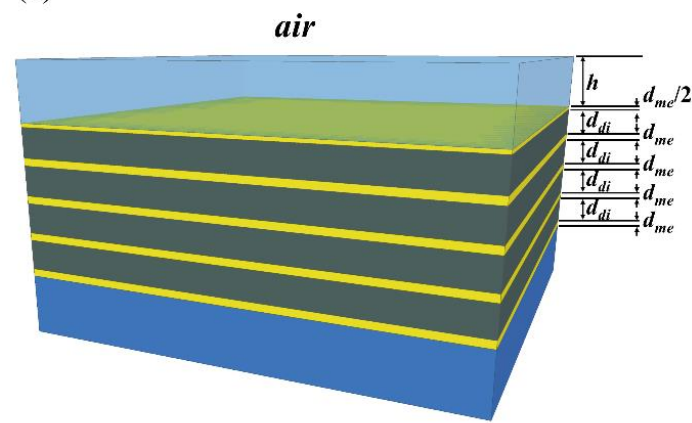

Silver $(\mathrm{Ag})$

Silicon Dioxide $\left(\mathrm{SiO}_{2}\right)$

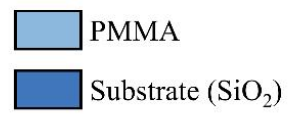

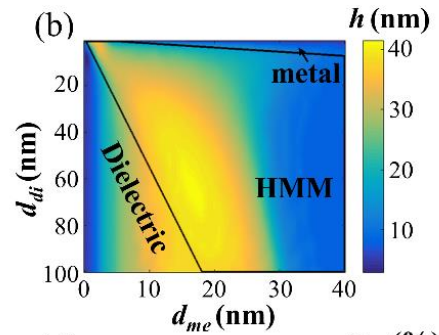

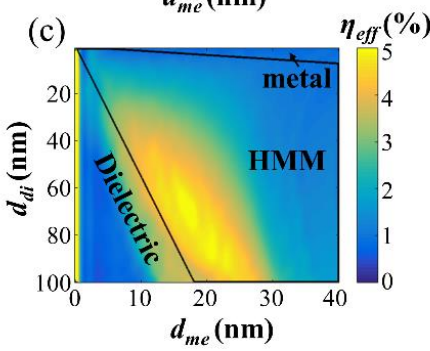

Figure 1.2. (a) $\mathrm{HMM}$ consisting of 9 alternating $\mathrm{Ag} / \mathrm{SiO}_{2}$ layers on top of a $\mathrm{SiO}_{2}$ substrate. The PMMA cover layer with thickness $h$ is positioned on top of the HMM. The substrate and air cover layers (above the PMMA) are assumed semi-infinite. The (b) and (c) maps show the values of $h$ and $\eta_{e f f}$, respectively, as function of the metal $\left(d_{m e}\right)$ and dielectric $\left(d_{d i}\right)$ thicknesses required to achieve $P=10$. The black lines in (b) and (c) separate the regions where the stack behaves as a metal, a HMM and a dielectric, according to [101]. 


\section{FOLHA DE JULGAMENTO}

Candidato: Engenheiro ACHILES FONTANA DA MOTA.

Título da tese: "Modelo e análise de metamateriais hiperbólicos para o controle da emissão espontânea e eficiência de emissores quânticos"

Data da defesa: 11/02/2019

Comissão Julgadora:

Resultado:

Prof. Associado Ben Hur Viana Borges

APROVADO

(Orientador)

(Escola de Engenharia de São Carlos/EESC)

Profa. Dra. Heide Ottevaere

APROVADO

(Vrije Universiteit Brussel)

Profa. Dra. Wendy Meulebroeck

APROVADO

(Vrije Universiteit Brussel)

Prof. Associado Philippe Wilhelm Courteille

APRO VNDO (Instituto de Física de São Carlos/IFSC)

Prof. Dr. Thiago Pedro Mayer Alegre

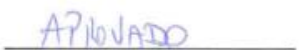

(Universidade Estadual de Campinas/UNICAMP)

Prof. Dr. Gustavo Silva Wiederhecker

(Universidade Estadual de Campinas/UNICAMP)

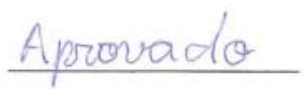

Coordenador do Programa de Pós-Graduação em Engenharia Elétrica:

Prof. Titular Luis Fernando Costa Alberto

Presidente da Comissão de Pós-Graduação:

Prof. Titular Luis Fernando Costa Alberto 



\section{Acknowledgements}

Firstly, I would like to thank my fiancé Renata for all her love, encouragement, and total support during my doctoral journey. I also would like to thank my parents Ailton and Isa Mara as well as my sister Isabely and my brother Thiago. Their patient and constant support were crucial for me during my PhD.

I would like to thank my advisor Ben-Hur Viana Borges, who has been contributing to my academic development since my graduation. I am grateful for all discussions, guidance, confidence, helpfulness and advices through this long and challenging path.

I would like to thank my advisors at VUB, Heidi Ottevaere and Wendy Meulebroeck, for receiving, guiding, teaching and giving me advices during the year I spent in Brussels, Belgium.

I would like to thank the Professors Fernando Lisboa Teixeira, John Weiner and Emiliano Martins Rezende for all their contributions to my work.

I would like to express my gratitude to my great friends:

my friends at USP: Anderson, Augusto, Arturo, Daniel Marchesi, Daniel Mazulquim, Heinz, Leone, Pedro, Thiago, Vinicius, Matheus, Rodrigo, Rafael, Talita, Larissa, and Leone.

my colleagues at VUB: Adnieszka, Benjamin, Fei, Koen, Lien, Marcin, Majorie, Mulham, Qing, Raymundo, Robert, Sid, Sophie, Tigran, and Urszula.

Finally, I would like to thank the funding agencies:

- $\quad$ Coordenação de Aperfeiçoamento de Pessoal de Nível Superior - Brasil (CAPES) - Finance code 001; PDSE (process number: 881.133554/2016-1);

- $\quad$ Erasmus Bemundus (process number: BM16DM1006).

- The computational support of the Núcleo de Apoio a Óptica e Fotônica (NAPOFUSP) and the computing time provided on the DaVinci cluster supported by the Center for Research Computing (Rice University) and Superintendência de Tecnologia da Informacão da Universidade de São Paulo. 



\section{Abstract}

MOTA, A. F. Modeling and analysis of hyperbolic metamaterials for controlling the spontaneous emission rate and efficiency of quantum emitters. Thesis (Ph. D.), São Carlos School of Engineering, University of São Paulo, São Carlos, 2019.

In the past few years, intensive research efforts have been devoted to studying new approaches to controlling the photon emission of quantum emitters (QEs), especially for telecommunication applications. These approaches rely on tailoring the QE's radiation, usually assessed via well-known figures-of-merit such as lifetime $(\tau)$ and quantum efficiency $(\eta)$. Controlling the QE's photon emission is important because the faster its photons are emitted, the greater is the number of times it returns to the excited state per second. Therefore, it is crucial to create additional decay channels to reduce $\tau$, which necessarily requires increasing the Purcell factor $(P)$. One of the most promising approaches to increase $P$ involves a new class of metamaterials, known as hyperbolic metamaterials (HMM). This class of materials exhibits pronounced anisotropy, with the parallel and perpendicular permittivity tensor elements (with respect to the anisotropy axis) presenting opposite signs, resulting in an open hyperboloidal isofrequency surface (IS). This unusual IS shape leads to the most outstanding feature of HMMs, namely, the existence of photonic modes with wavenumber $(k)$ much larger than those in free-space $\left(k_{0}\right)$, known as high- $k$ modes. By engineering these modes, it is possible to manipulate the HMM photonic density of states (PDoS), thus controlling the QE's radiation parameters.

The simplest approach to designing HMM is by means of a planar stack of alternating thin metal and dielectric layers. However, the finite thickness of these layers induces spatial dispersion, making the extraction of effective parameters (homogenization) of these media a challenging task. In this context, we propose in this thesis a new constitutive parameter retrieval approach that takes spatial dispersion into account for all electromagnetic parameters of the medium. We demonstrate that the real part of the dispersion curve flattens out (correspondingly with a large imaginary part) because of the absence of propagating modes inside the metamaterial. This flat region is strongly dependent on the layer thicknesses and is a direct manifestation of spatial dispersion. Moreover, we demonstrate that the QE's lifetime calculation is overestimated if this effect is not taken into account in the homogenization procedure, which is detrimental for telecommunication applications.

Moreover, we demonstrate how to enhance $\mathrm{P}$ by a factor greater than 100 with the use of HMMs. However, most of the QE dissipated power couples into the HMM as high- $k$ modes (which do not propagate in free-space). Therefore, the energy is thermally dissipated inside the HMM with a consequent reduction of $\eta$. Some authors have resorted lano-patterned HMMs (NPHM) to convert the high- $k$ modes into free-space modes o) aiming at increasing $\eta$. However, much of the NPHMs designs still rely on 
computationally costly three dimensional (3D) numerical simulations. Thus, we also propose in this thesis a new semi-analytical method to model, both in two- and threedimensions (2D and 3D, respectively), the radiation emission of QEs interacting with nano-patterned structures. The low computational cost of this method makes it attractive for mapping $P$ and $\eta$ as function of the QE and NPHM relative position. This mapping is a helpful tool to understand the decay behavior of the whole system since QEs are arbitrarily distributed and oriented inside the NPHM. The analytically calculated decay curve allows the system's effective quantum efficiency $\left(\eta_{\text {eff }}\right)$ and Purcell factor $\left(P_{\text {eff }}\right)$ to be directly obtained assuming multiple arbitrarily distributed electromagnetic sources.

In this sense, we propose here a new procedure to optimize the NPHM geometrical parameters to maximize $\eta_{\text {eff }}$ while achieving the desired $P_{\text {eff }}$. We apply the proposed model to an NPHM composed of nine $\mathrm{Ag} / \mathrm{SiO}_{2}$ layers, with the polymer host layer embedded with Rhodamine 6G, to maximize $\eta_{\text {eff }}$ for a specified tenfold increase of $P_{\text {eff. }}$. This procedure allowed $\eta_{\text {eff }}$ to be increased by $69 \%$ and $170 \%$ for one- and two-dimensional nanopatterning, respectively. Moreover, the time required to build the $P$ and $\eta$ maps (used in the calculation of the decay behavior) is reduced by approximately $96 \%$ when compared to those numerically calculated via FDTD. This procedure paves the way to the realization of new high-speed and efficient light sources for telecommunication applications.

Key-words: Hyperbolic Metamaterials, spontaneous emission, Purcell factor, quantum emitters, homogenization, electromagnetic modelling, quantum efficiency. 


\section{Resumo}

MOTA, A. F. Modelo e análises de metamateriais hiperbólicos para o controle da taxa de emissão espontânea e eficiência de emissores quânticos. Tese (Doutorado), Escola de Engenharia de São Carlos, Universidade de São Paulo, São Carlos, 2019.

Nos últimos anos, intensivo esforço tem sido devotado para o estudo de novas método para o controla da missão de fótons de emissores quânticos (EQs), especialmente para aplicações em telecomunicações. Estes métodos dependem da adaptação da radiação dos EQs, geralmente avaliadas por meio das bem conhecidas figuras de mérito, como o tempo de meia vida $(\tau)$ e a eficiência quântica $(\eta)$. O controle da emissão de fótons é importante pois quanto mais rápido os fótons são emitidos, maior é o número de vezes que o $\mathrm{EQ}$ retorna ao seu estado excitado por segundo. Portanto, é crucial criar canais de decaimento adicionais para reduzir $\tau$, o que necessariamente requer o aumento do fator de Purcell $(P)$. Uma das abordagens mais promissoras para aumentar $P$ envolve uma nova classe de metamateriais, conhecida como metamateriais hiperbólicos (MHs). Esta classe de materiais apresenta pronunciada anisotropia, onde os elementos paralelo e perpendicular do tensor de permissividade (em relação ao eixo de anisotropia) apresentam sinais opostos, resultando em uma superfície de isofrequência (SI) hiperboloidal aberta (IS). Essa forma incomum de SI leva à característica mais marcante dos MHs, a existência de modos fotônicos com número de onda $(k)$ muito maior do que aqueles no espaço livre $\left(k_{0}\right)$, conhecidos como modos alto- $k$. Ao manipular esses modos, é possível manipular a densidade de estados fotônicos (DES) dos MHs, controlando assim os parâmetros de radiação do $\mathrm{QE}$.

A abordagem mais simples para a criação de MHs é por meio de uma pilha plana de camadas metálicas e dielétricas alternadas. Entretanto, a espessura finita dessas camadas induz a dispersão espacial, tornando a extração de parâmetros efetivos (homogeneização) destes meios uma tarefa desafiadora. Neste contexto, propomos nesta tese uma nova abordagem de recuperação de parâmetros constitutivos a dispersão espacial de todos os parâmetros eletromagnéticos do meio é levada em consideração. Nós demonstramos que a parte real da curva de dispersão se aplaina (correspondentemente com uma grande parte imaginária) devido à ausência de modos propagantes dentro do metamaterial. Esta região plana é fortemente dependente das espessuras das camadas e é uma manifestação direta da dispersão espacial Além disso, nós mostramos que se a dispersão espacial não for corretamente considerada no processo de homogeneização, o tempo de meia vida do EQ pode ser superestimado, o que é prejudicial para aplicações de telecomunicações.

Além disso, demonstramos como melhorar $P$ por um fator maior que 100 com o uso de MHs. a maior parte da potência dissipada pelos EQs são acopladas nos MHs como modos de alto-k (que não se propagam no espaço livre). Portanto, a energia é dissipada termicamente no interior do $\mathrm{MH}$, resultando em uma redução de $\eta$. Alguns autores 
recorreram a MHs nano-estruturados (MHNE) para converter os modos alto- $k$ em modos de espaço livre $\left(k \leq k_{0}\right)$ visando o aumento de $\eta$. No entanto, muitos dos projetos do NPHM ainda dependem de simulações numéricas tridimensionais (3D) computacionalmente dispendiosas. Assim, também propomos nesta tese um novo método semi-analítico para modelar, tanto em duas como em três dimensões (2D e 3D, respectivamente), a emissão de radiação de EQs interagindo com estruturas nano-estruturadas. $\mathrm{O}$ baixo custo computacional deste método faz com que seja atrativo para o mapeamento de $P$ e $\eta$ em função da posição relativa do EQ e do MHNE. Esse mapeamento é uma ferramenta útil para entender o comportamento de decaimento de todo o sistema, já que os EQs são arbitrariamente distribuídos e orientados dentro do MHNE. A curva de decaimento calculada analiticamente permite que a eficiência quântica efetiva do sistema $\left(\eta_{\text {eff }}\right)$ e o fator de Purcell $\left(P_{e f f}\right)$ sejam obtidos diretamente, assumindo múltiplas fontes eletromagnéticas arbitrariamente distribuídas.

Neste sentido, propomos aqui um novo procedimento para otimizar os parâmetros geométricos do MHNE visando a maximização de $\eta_{\text {eff }}$ enquanto $P_{\text {eff }}$ é aumentado à um valor desejado. Aplicamos o modelo proposto a um MHNE composto por nove camadas de $\mathrm{Ag} / \mathrm{SiO} 2$, com a camada de polímero embutida com Rodamina 6G, visando maximizar $\eta_{\text {eff }}$ para um aumento de dez vezes de $P_{\text {eff. }}$. Este procedimento permitiu que o $\eta_{\text {eff }}$ fosse incrementado em $69 \%$ e $170 \%$ para nano-estruturas uni e bidimensionais, respectivamente. Além disso, o tempo necessário para construir os mapas $P$ e $\eta$ (utilizados no cálculo da curva de decaimento) é reduzido em aproximadamente $96 \%$ quando comparado com os calculados numericamente via FDTD. Este procedimento abre caminho para o desenvolvimento de novas fontes de luz de alta velocidade e eficiência para aplicações de telecomunicações.

Palavras-chave: Metamateriais hiperbólicos, emissão espontânea, fator de Purcell, emissores quânticos, homogeneização, modelagem eletromagnética, eficiência quântica. 


\section{Table of Contents}

ACKNOWLEDGEMENTS

Abstract iii

RESUMO ..v

TABLE OF CONTENTS ...................................................................................................... vii

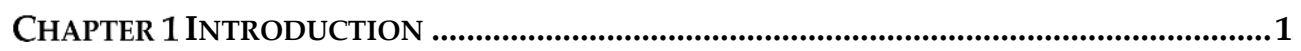

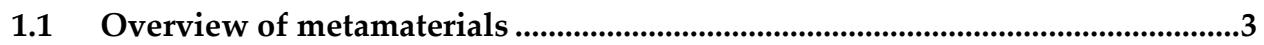

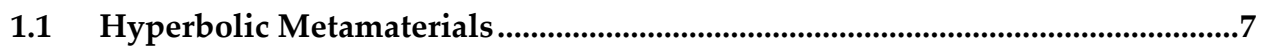

1.1.1 Homogenization of metamaterials ……….....................................................

1.1.2 Manipulating radiation parameters of QE....................................................11

1.1.3 Grating-assisted radiation emission of quantum emitters .............................14

1.1.4 General approach for calculating the radiation emission of QEs inside bounded hyperbolic metamaterials ...............................................................17

1.2 Objectives of this thesis ..............................................................................................17

1.3 Organization of the Thesis ........................................................................................18

CHAPTER 2 HOMOGENIZATION OF UNIAXIAL ANISOTROPIC METAMATERIALS ........... 21

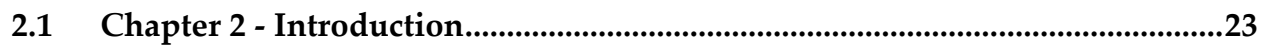

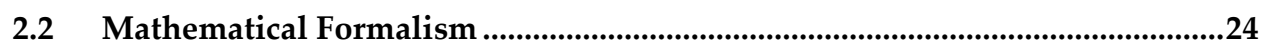

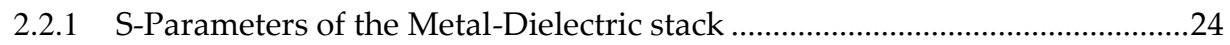

2.2.2 S-Parameters of the Anisotropic Medium .......................................................26

2.2.3 Extraction of the Constitutive Parameters from the S-Parameters of the Slab

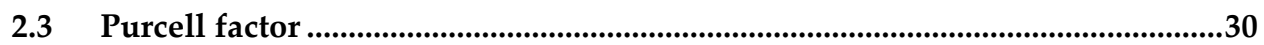

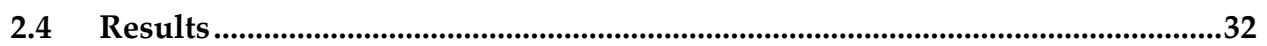

2.4.1 Influence of the Dielectric Thickness ..................................................................32

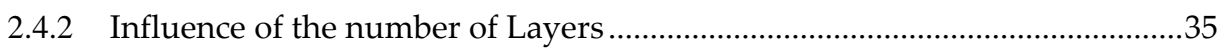

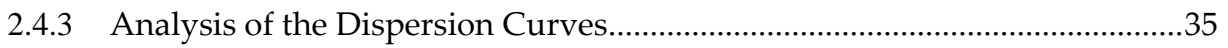

2.5 Calculation of the Purcell Factor .............................................................................41

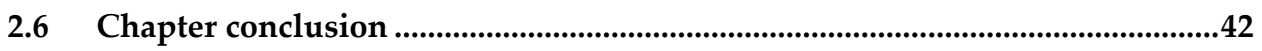

ChaPTER 3 ENHANCING THE PURCELl factor USING HMM ......................................45 


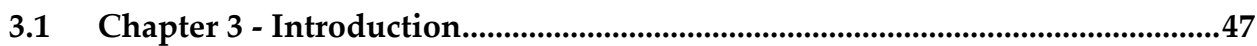

3.2 Analysis of the Hyperbolic Metamaterial ..........................................................48

3.3 Single Cy7 molecule embedded in a PMMA layer................................................52

3.4 Homogenous distribution of $\mathrm{Cy} 7$ inside the PMMA layer ................................54

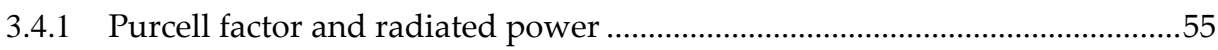

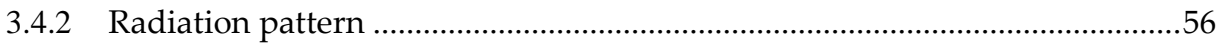

3.4.3 Decay curve ..............................................................................................5

3.5 Influence of the PMMA thickness in system's Purcell factor and quantum efficiency ..................................................................................................................................58

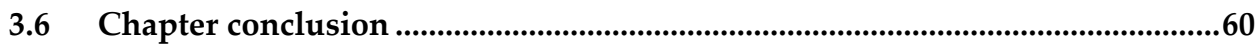

CHAPTER 4 DESIGN AND ANALYSIS OF GRATING-ASSISTED RADIATION EMISSION

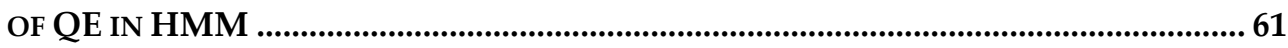

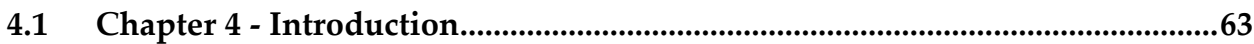

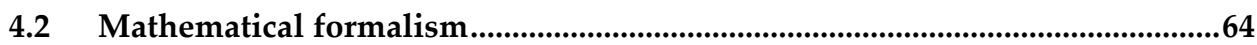

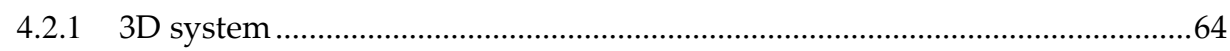

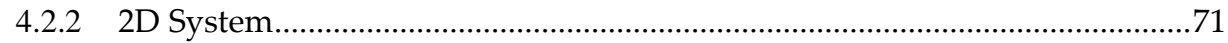

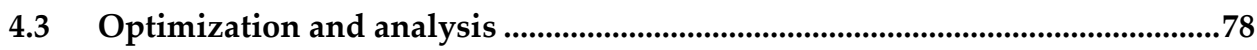

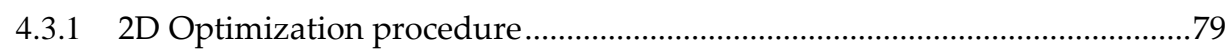

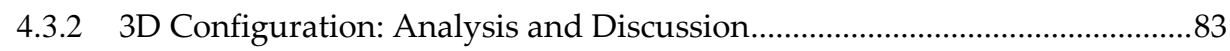

4.4 Comparison between HMM with and without metallic gratings.....................87

4.5 Computation improvement of our method ..............................................................89

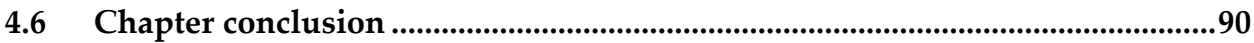

CHAPTER 5 SEMI-ANAlytical MODEling OF QES EMBEDDED IN NANO-

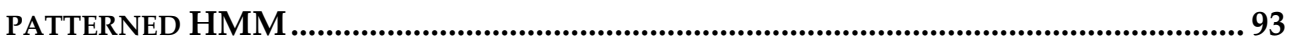

5.1 Chapter 5 - Introduction..............................................................................................95

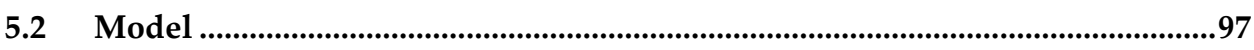

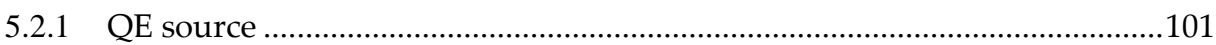

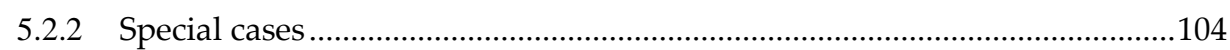

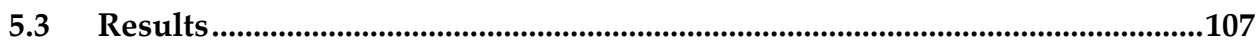

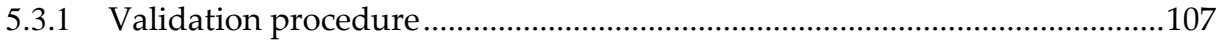

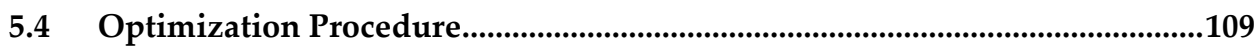

5.4.1 Step 1: 3D-SAM optimization of a non-patterned HMM.............................109

5.4.2 Step 2: NPHM period and fill-factor optimization with the 2D-SAM ........111

5.4.3 Step 3: NPHM fine tuning with the 3D-SAM ...............................................112

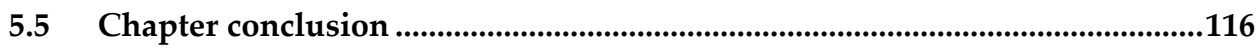


6.1 Main achievements ....................................................................................................121

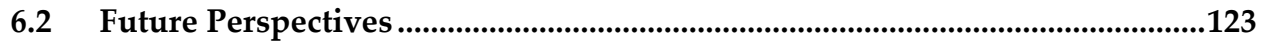

6.2.1 Nano-patterned hyperbolic metamaterials for telecommunication

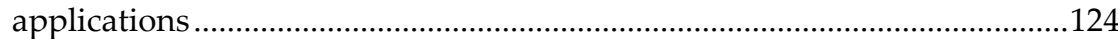

6.2.2 Nano-patterned hyperbolic metamaterials for sensing applications ..........125

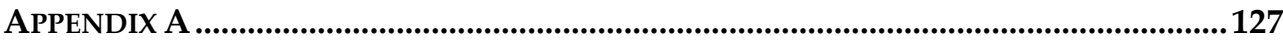

A.1. Homogenization Procedure based on a Drude-Lorentz Dispersive Model for

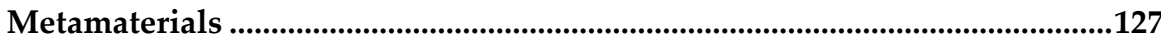

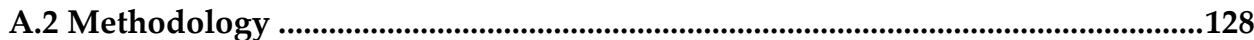

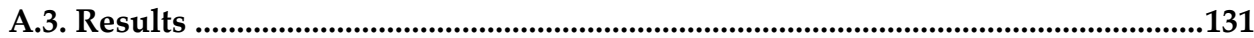

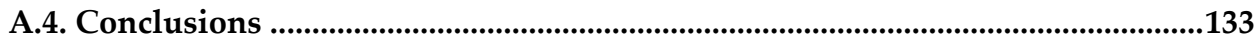

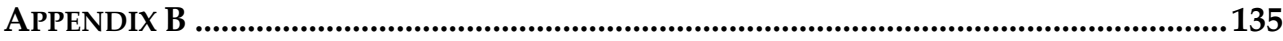

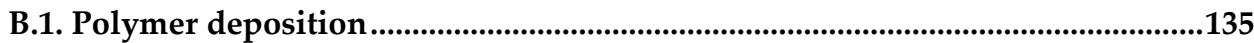

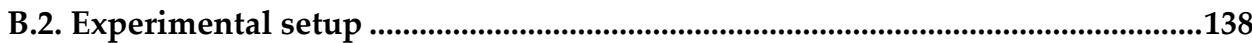

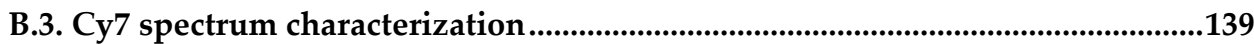

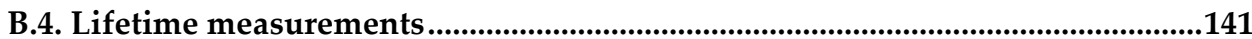

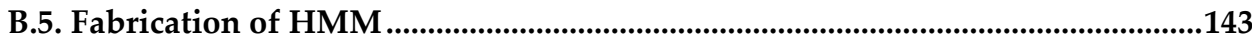

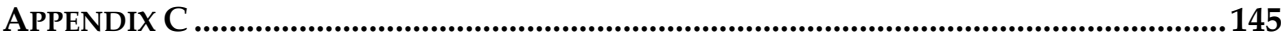

C.1. Lumerical 2D-FDTD Simulation........................................................................145

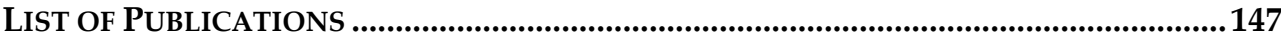

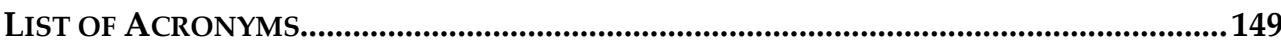

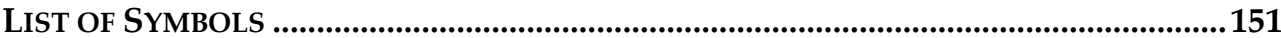

Latin - Roman letters ..............................................................................................................151

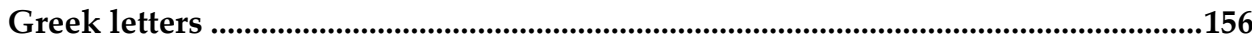

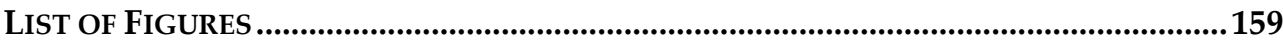

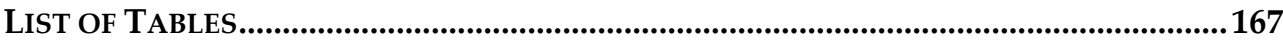

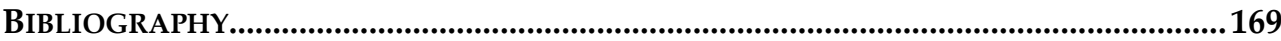

ANNEX 1 - PHYSICAL REVIEW COPYRIGHTS PERMISSION ...........................................180 
ANNEX 2 - ACS PHOTONICS COPYRIGHTS PERMISSION............................................. 183

ANNEX 3 - MOTL COPYRIGHTS PERMISSION ......................................................... 185

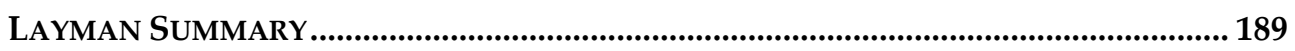




\section{Chapter 1 \\ Introduction}




\section{Chapter 1 - Summary}

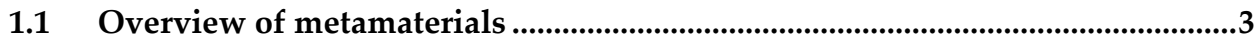

1.1 Hyperbolic Metamaterials ............................................................................................

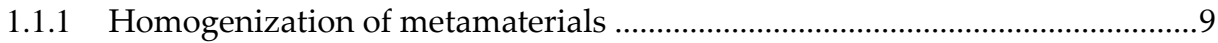

1.1.1.1 Homogenization of hyperbolic metamaterials..........................................10

1.1.2 Manipulating radiation parameters of QE.....................................................11

1.1.3 Grating-assisted radiation emission of quantum emitters ..............................14

1.1.4 General approach for calculating the radiation emission of QEs inside bounded hyperbolic metamaterials ................................................................17

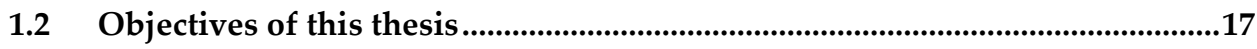

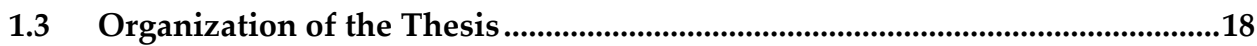




\subsection{Overview of metamaterials}

Artificially structured materials (or metamaterials) with pre-designed electromagnetic properties [1]-[5] have become a new paradigm in the design of electromagnetic structures at all frequency ranges, as predicted by Pendry et al. [6]. The easy manipulation of the electromagnetic properties of these media has rendered them ideal both for scientific and technological applications [1], [6]-[13]. As described by Smith et al. [12], the permittivity $(\varepsilon)$ and permeability $(\mu)$ of these media do not need to arise from the response of elementary cells, as in ordinary materials. In metamaterials, these cells are created via a collection of macroscopic objects whose dimension and spacing are much smaller than the wavelength. Consequently, effective (or homogeneous) $\varepsilon$ ( $\left.\varepsilon_{\text {eff }}\right)$ and $\mu$ ( $\left.\mu_{\text {eff }}\right)$ arise from the scattering properties of these objects [12] (or inclusions), causing metamaterials to exhibit properties that are difficult or even impossible to find in nature. The authors in [12] also provide an interesting comparison between the homogeneous behavior of metamaterials and atomic structures as illustrated in Figure 1.1. In this example it is assumed light is propagating through an array of elementary cells of glass (a) and in an array of macroscopic atoms in a metamaterial (b). Since the wavelength of light is hundreds of times larger than the cell size, the influence of an individual cell on the light wave is neglectable. In fact, the wave is influenced by a large ensemble of elementary cells that are averaged, resulting in the macroscopic electromagnetic parameters, $\varepsilon$ and $\mu$ [14]. This analysis can be extended to metamaterials as well because, from an electromagnetics point of view, any tridimensional array of artificial structures (or macroscopic atoms) with dimensions much smaller than the wavelength (typically $<\lambda / 10$ ) can be seen as a homogeneous material, as shown in Figure 1.1 (b). Therefore, just by changing the geometrical parameters of the "macroscopic atom", it is possible to manipulate the effective parameters $\varepsilon_{\text {eff }}$ and $\mu_{\text {eff. }}$.

The idea of manipulating electromagnetic parameters of a medium dates back to the end of the $19^{\text {th }}$ century, when J. C. Bose used this concept to rotate the polarization state of microwave plane waves using twisted fibers [15]. This concept was further explored by K. Lindman, who, in 1920, embedded randomly oriented metallic twisted wires (helices) with dimensions much smaller than the wavelength $\left(\lambda_{0}\right)$ in a dielectric host medium to

(a)

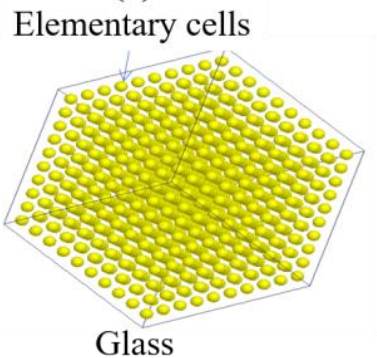

(b)

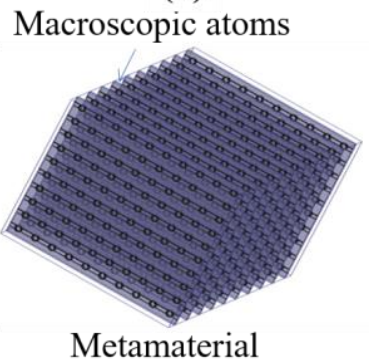

Figure 1.1. (a) Array of elementary cells in a glass (a) and (b) array of macroscopic atoms of a metamaterial (Based on [13]). 
create an isotropic artificial chiral medium [16]. Bose and Lindman were pioneers exploiting the artificial media concept [1].

Nevertheless, the first artificial dielectric material without chiral properties was proposed by W. E. Kock in 1948 [17] who designed microwave lenses using periodic and subwavelength spheres, disks and strips to control the lenses' material refractive index $(n=$ $\sqrt{\varepsilon \mu}$ ). The velocity of the electromagnetic wave propagating through the lenses was easily controlled by the size and periodicity of the sub-wavelength elements. This control allowed the design of microwave lenses with performance similar to conventional lenses using artificial dielectric metamaterials [17]. Kock's concept was later explored by J. Brown in 1952 through an array of small metallic wires to produce a medium with refractive index smaller than one [18]. In 1962, W. Rotman used the wire media to manipulate the plasma frequency in order to achieve negative $\varepsilon_{\text {eff }}[19]$. Later, G. Thompson exploited this idea to manipulate the medium's magnetic response $\left(\mu_{e f f}\right)$ using ferrite wires [20]. The ferrite magnetic response allowed $\mu_{\text {eff }}$ to be controlled (including negative values of $\mu_{e f f}$ ) manipulating the wires periodicities and dimensions [20]. Nevertheless, the ferrite wire medium proved to be highly lossy, which hindered its application for practical purposes.

A major breakthrough in metamaterials research occurred in 1967, when V. Veselago theoretically studied the behavior of electromagnetic waves inside a medium with simultaneously negative $\varepsilon$ and $\mu$ [21]. Veselago showed that if both $\varepsilon$ and $\mu$ are negative, the refractive index $(n)$ is also negative. Consequently, the wavevector $(\mathbf{k})$ and the Poynting (S) vector are in opposite direction, as opposed to conventional materials. Furthermore, the negative $n$ of such medium results in reverse Doppler and Cherenkov effects, as well as a negative refraction, which has been used by Veselago to postulate the visionary flat lens [21].

Although revolutionary, the concept of a medium with negative $n$ as proposed by Veselago could not be experimentally implemented at that time because all known media with negative $\mu$ were highly lossy [20]. Many researches tried to create such media, but a major advance on the subject only occurred 29 years after Veselago's publication. In 1996, inspired by the Veselago's flat lens, J. B. Pendry et al. used periodic thin metallic wires to bring the medium's plasma frequency $\left(f_{p}\right)$ down into the GHz range [22]. Pendry showed that $f_{p}$ could be tuned by changing the wire dimension and periodicity, resulting in a wide-range of permittivity values, from negative $\left(f<f_{p}\right)$ to positive values $\left(f>f_{p}\right)$ [22]. The solution for negative $\mu$ was proposed three years later also by Pendry's group [23], where the manipulation of $\mu_{\text {eff }}$ was achieved with two concentric metallic split rings with dimensions much smaller than the free space wavelength $\lambda_{0}$. These structures, also known as split-ring resonators (SRR), can be periodically arranged (as shown in Figure 1.1 (b)) and designed to behave as magnetic dipoles with resonance frequency $\left(f_{r}\right)$ at the microwave regime. At $f_{r}, \mu_{e f f}$ changes its sign from positive to negative with low losses (solving the attenuation problem of [20]). Therefore, $\mu_{\text {eff }}$ could be tuned from negative to positive values just by changing the SRR geometrical parameters [23].

One year after Pendry's proposal on how to design a medium with negative $\varepsilon$ or $\mu, \mathrm{D}$. R. Smith et al. combined the two approaches to create a medium with $\varepsilon$ and $\mu$ simultaneously 
negative, and consequently negative index of refraction [24]. In 2001, R. A. Shelby et al. experimentally confirmed Smith's theoretical predictions producing the first metamaterial with negative index of refraction [25]. Although their goal was to present a metamaterial with negative $n$, Shelby's results also demonstrated that with Pendry's approach it would be possible, in principle, to artificially create materials with any desired electromagnetic properties, even properties not found in nature, such as negative $n$.

The impressive degree-of-freedom allowed by the manipulation of the medium's effective response ( $\varepsilon_{\text {eff }}$ and $\mu_{\text {eff }}$ ) continues to attract a great deal of attention to the metamaterials field, particularly in applications such as antenna designs [26]-[28], invisibility cloaks [10], [11], [29], [30], lenses [9], biosensors [7], [31]-[33], imaging [34][36], control of radiation emission [37]-[40], just to cite a few. The increased performance of these applications is due to the ability of individually manipulating the metamaterials parameters $\varepsilon$ and $\mu$, from positive to negative values. The wide range of possible material properties allow metamaterials to be organized in five main categories, as seen in Figure 1.2. The details of each metamaterial category are given bellow:

- Double Positive Material or DPS $(\varepsilon>0, \mu>0)$ : a medium where both $\varepsilon$ and $\mu$ are greater than zero, corresponding to the first quadrant in Figure 1.2. Most of the natural materials are classified as DPS (e.g., dielectric). However, metamaterials allow tailoring $\varepsilon$ and $\mu$ to any desired value, including those not found in natural materials.

- Epsilon Negative Material or ENG $(\varepsilon<0, \mu>0)$ : a medium with $\varepsilon$ smaller than zero and $\mu$ greater than zero. Most metals present negative $\varepsilon$ (e.g., silver, gold and aluminum) at frequencies bellow $f_{p}$, which are normally at the optical regime. Metamaterials allow the plasma frequency to be tuned so that $\varepsilon$ can be controlled even in the low-frequency regime, such as microwave and infrared [22]. EPS metamaterials are obtained, for instance, by means of wire media [22], as shown in the second quadrant of Figure 1.2.

- $\quad \mathrm{Mu}$ Negative Material or MNG $(\varepsilon>0, \mu<0)$ : a medium with $\mu$ smaller than zero and $\varepsilon$ greater than zero. MNG are found in some gyrotropic and naturally occurring magnetic materials. Metamaterials allow $\mu$ to be varied via tuning of the magnetic dipole resonance of its constituents, such as SRRs [23] as shown in the fourth quadrant in Figure 1.2.

- Double Negative Material DNG $(\varepsilon<0, \mu<0)$ : a medium with both $\varepsilon$ and $\mu$ smaller than zero. Until this date, materials with negative $\varepsilon$ and $\mu$ have not been found in nature. DNG metamaterials, resulting in negative $n$, are applied in many applications, such as perfect lenses [9], pulse dispersion control [41], [42], and negative refraction (inversion of Snell's law) [43]. For instance, this kind of metamaterial can be realized by combing wire media $(\varepsilon<0)$ with SRRs $(\mu<0)$, as shown in the third quadrant in Figure 1.2. 


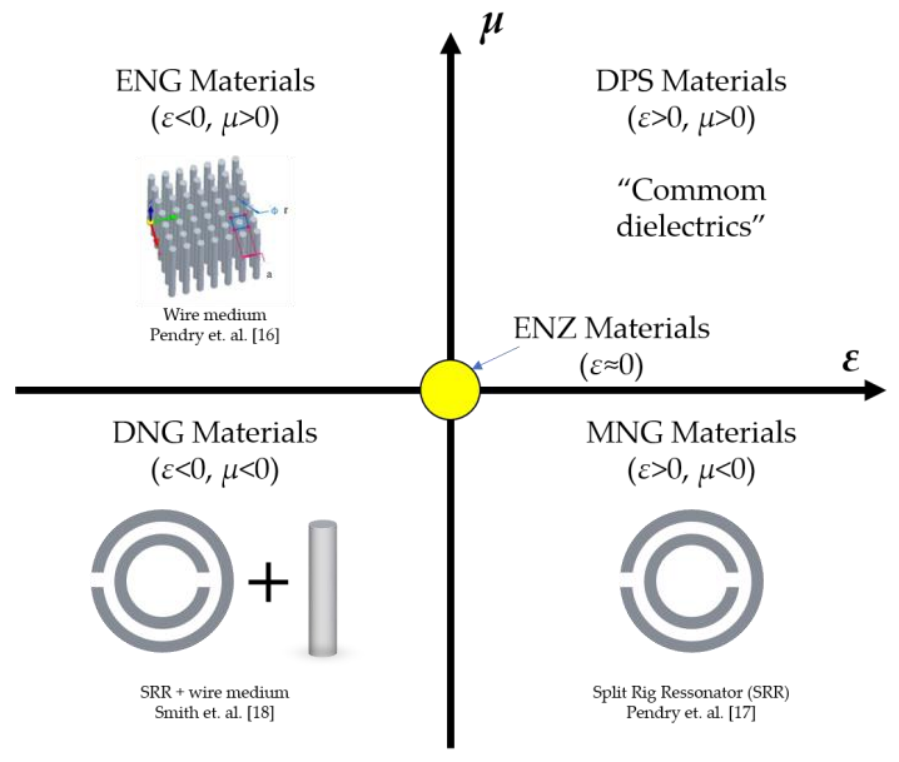

Figure 1.2. Metamaterials classification (Adapted from [1]).

- Epsilon near zero or ENZ ( $\varepsilon \approx 0)$ : a medium with $\varepsilon$ close to zero. This class of metamaterials is very interesting because if $\varepsilon$ is close to zero, $n$ also gets close to zero. Consequently, light propagates with almost no phase advance [44][46]. This feature is being used in the literature for enhancing the spontaneous emission of quantum emitters [40], [46], supercoupling [47], antenna designs [48], among others.

The metamaterials classification shown in Figure 1.2 was proposed by R. W. Ziolkowski and N. Engheta in 2006 [1] and is only applied to isotropic metamaterials. Nonetheless, new types of metamaterials with anisotropic properties have been proposed since then. One special class of such metamaterials is the so called hyperbolic metamaterials (HMM) [49], which is the main subject of this thesis. This class of metamaterials has attracted a great deal of attention over the past years, particularly at optical frequencies [37] for its ability to produce negative refraction [38], [50], hyperlenses [51]-[53], biosensors [32], broadband absorbers [54] and, more importantly, to increase the photonic density of states of quantum emitters [37], [40], [55]-[58]. HMMs are extremely anisotropic, having opposite signs for the parallel and perpendicular components of their permittivity tensor. Although the literature is mostly focused on electric HMMs, it has been recently proposed a magnetic HMM, a material in which the permeability tensor has elements with opposite signs [59]. In this thesis we focus only on the physical properties ruling the behavior of electric HMMs and their applications. Therefore, the next session treats the most important aspects of these media and how this thesis has contributed to increase the knowledge in this research area. 


\subsection{Hyperbolic Metamaterials}

As mentioned in the previous section, HMMs are extremely anisotropic, having opposite signs for the parallel $\left(\varepsilon_{\|}\right)$and perpendicular $\left(\varepsilon_{\perp}\right)$ components of their permittivity tensor (ع) [37]. Consequently, these metamaterials present metallic behavior in a given direction (the direction presenting negative permittivity) and dielectric behavior in the other (the direction presenting positive permittivity). The HMM permittivity tensor $(\varepsilon)$ is diagonal and written as

$$
\varepsilon=\left[\begin{array}{ccc}
\varepsilon_{x} & 0 & 0 \\
0 & \varepsilon_{y} & 0 \\
0 & 0 & \varepsilon_{z}
\end{array}\right]
$$

where $\varepsilon_{x}, \varepsilon_{y}$ and $\varepsilon_{z}$ are the permittivity components in $x, y$, and $z$ directions, respectively. The term hyperbolic metamaterial is derived from the hyperbolic (as opposed to ellipsoidal or spherical) dispersion profile of its isofrequency surface (IS). Normally, HMMs are designed in such a way as to present symmetry in two planes [37] and, consequently, two elements of $\varepsilon$ are equal. If the symmetry planes are $x-z$ and $y-z$, we have $\varepsilon_{x}=\varepsilon_{y}$, which are defined as $\varepsilon_{\perp}$, since these are the elements perpendicular to the HMM optical axis. Analogously, $\varepsilon_{z}$ is parallel to the optical axis and defined as $\varepsilon_{\|}$. Applying these definitions into the Maxwell equations, the HMM's IS (or dispersion surface) is written as [37]

$$
\frac{k_{x}^{2}+k_{y}^{2}}{\varepsilon_{\|}}+\frac{k_{z}^{2}}{\varepsilon_{\perp}}=k_{0}^{2},
$$

where $k_{x}, k_{y}$ and $k_{z}$ are the wavevector components in $x, y$ and $z$ directions, respectively, $k_{0}$ is the free space wavenumber. From (1.2), if $\varepsilon_{\|} \varepsilon_{\perp}>0$ the IS is an ellipsoid (Figure 1.3 (a)), while if $\varepsilon_{\|} \varepsilon_{\perp}<0$ it becomes a one- or two-sheeted hyperboloid. If $\varepsilon_{\perp}>0$ and $\varepsilon_{\|}<0$, the IS surface is a two-sheeted hyperboloid (Figure $1.3(\mathrm{~b})$ ), while if $\varepsilon_{\perp}<0$ and $\varepsilon_{\|}>0$ it becomes a one-folded hyperboloid (Figure 1.3 (c)). This unusual IS shape leads to the most outstanding feature of HMMs, namely, the existence of photonic states with wavevectors (k, with modulus $k$ ) much larger than those in free space ( $\mathbf{k}_{0}$, with modulus $\left.k_{0}\right)$, resulting in a large photonic density of states (PDoS). This occurs because in conventional materials the IS is an ellipsoid where only finite values of $\mathbf{k}$ are possible, as seen in Figure $1.3(\mathbf{a})$; in contrast, an ideal hyperboloid-shaped IS has no upper cut-off for $\mathbf{k}$, meaning it supports an infinitely large number of high- $k$ modes [37], as seen in Figure $1.3(b, c)$.

The procedure to obtain metamaterials with hyperbolic dispersion is relatively simple. In order to achieve a negative permittivity in a given direction, the electrons must move freely along it as in metals [37]. In this sense, two different approaches are normally used to experimentally realize this profile. The first is through a planar stack of metal/dielectric layers (as can be seen in Figure 1.4 (a)) and the second is through wired medium (as seen in Figure 1.4 (b)) [38]. For both approaches, the dimensions of the structures must be much 


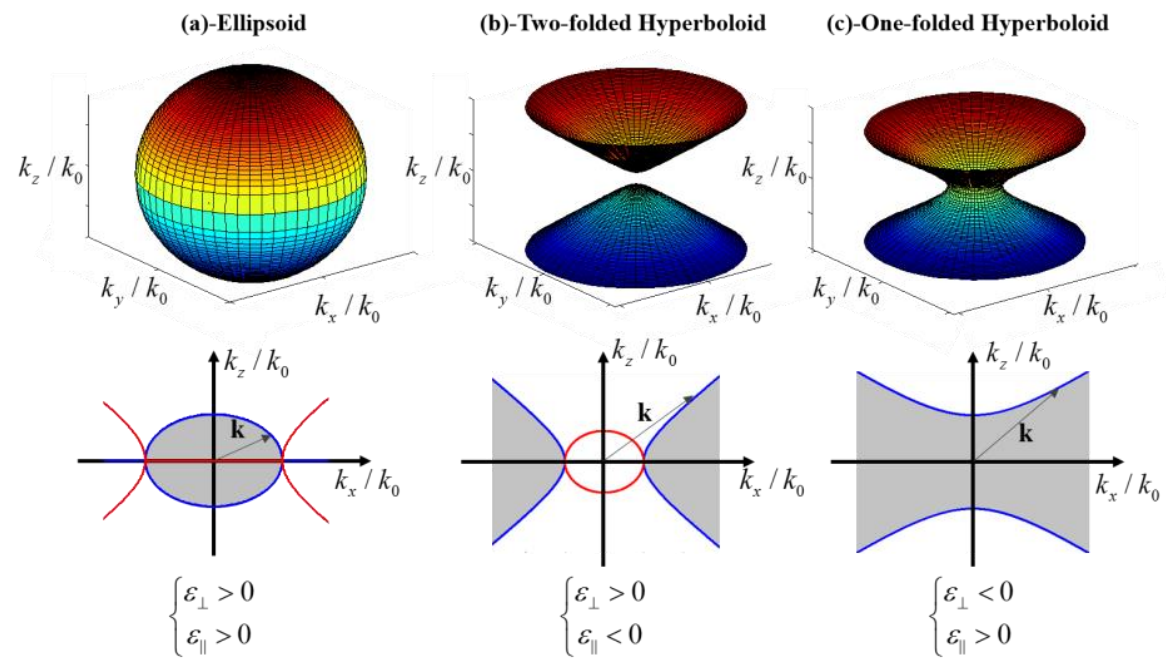

Figure 1.3. Isofrequency surfaces of (a) an isotropic dielectric $\left(\varepsilon_{\perp}=\varepsilon_{\|}\right)$, (b) a two-sheeted hyperboloid $\left(\varepsilon_{\perp}>0\right.$ and $\left.\varepsilon_{\|}<0\right)$, and (c) a one-folded hyperboloid $\left(\varepsilon_{\perp}<0\right.$ and $\left.\varepsilon_{\|}>0\right)$. The projection of each isofrequency surface on the plane $\mathrm{k}_{\mathrm{y}} / \mathrm{k}_{0}=0$ is shown at the bottom, where the real and imaginary parts are represented by blue and red lines, respectively. The grey area represents the region where it is possible to have low loss modes inside the stack.

smaller than the wavelength. Moreover, the shape of the hyperboloid (either single- or double-sheeted) can be easily controlled by an appropriate choice of wavelength, materials and geometry. Although simple to be realized, the extraction of effective parameters (homogenization) of metamaterials media has proved to be a challenging task [55]-[80]. In the next sub-section, we discuss the metamaterials homogenization procedures with focus on the peculiarities of HMMs.

(a) - Layered Medium

(b) - Wire Medium

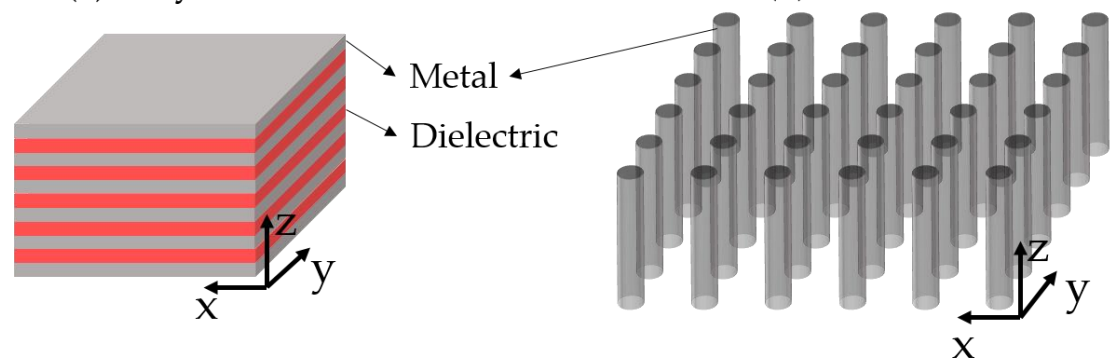

Figure 1.4. Hyperbolic Metamaterials. (a) metal-dielectric stack and (b) wire medium. 


\subsubsection{Homogenization of metamaterials}

We begin this subsection by first explaining the main concepts on how to retrieve the electromagnetic parameters of metamaterials, followed by the derivation of the homogenization procedure of HMMs. The extraction of effective parameters of metamaterial media is a difficult task and still the focus of many researches [48], [60], [69]-[78], [61], [79]-[85], [62]-[68]. The main difficulty arises from the complex geometry of the inclusions used to create these metamaterials, such as Smith's cell [25], omega cell [41], among others [61]-[65]. Most homogenization formalisms are derived from the metamaterials' scattering parameters (S-parameters) [55]-[68], which are easily obtained simulating just one cell (using periodic boundary conditions) in commercial softwares such as the High Frequency Structure Simulator (HFSS) [86] or Lumerical FDTD [87]. The central idea of most parameter retrieval procedures is that the metamaterial cell (as seen in Figure 1.5 (a)) must present the same electromagnetic behavior of an effective medium with $\varepsilon_{\text {eff }}$ and $\mu_{\text {eff }}$ (as seen in Fig Figure $1.5(\mathrm{~b})$ ). Consequently, both metamaterial and effective media must present the same reflection $\left(S_{11}\right)$ and transmission $\left(S_{21}\right)$ parameters, as shown in Figure 1.5. By manipulating Maxwell equations it is possible to determine which values of $\varepsilon_{\text {eff }}$ and $\mu_{\text {eff }}$ will produce the same $S_{11}$ and $S_{21}$ of the unit cell.

A critical issue regarding these procedures is that in certain frequency bands $\varepsilon_{\text {eff }}$ and $\mu_{\text {eff }}$ have no causal response [88] (a behavior also observed in [24], [68]). Moreover, the frequency dependence of the extracted $\varepsilon_{e f f}$ and $\mu_{e f f}$ vectors does not obey any known dispersive material model description, such as Drude and Lorentz dispersive models. As a result, it becomes very time consuming to simulate the homogenized structure with FDTD method due to the requirement of temporal convolutions of $\varepsilon_{\text {eff }}$ and $\mu_{\text {eff }}$ [89], [90]. Fortunately, the computational performance can be improved if one approximates $\varepsilon_{e f f}$ and $\mu_{\text {eff }}$ via Drude and Lorentz dispersive models, respectively [64], [91], [92]. This approach not only allows the temporal convolutions to be solved analytically, it also produces causal homogenized media.

We have also addressed the issue of non-causal response of homogenized metamaterials and proposed an improved homogenization procedure where the homogenized medium behaves precisely as the actual metamaterial [93]. This approach utilizes only Drude and

(a) - Metamaterial cell

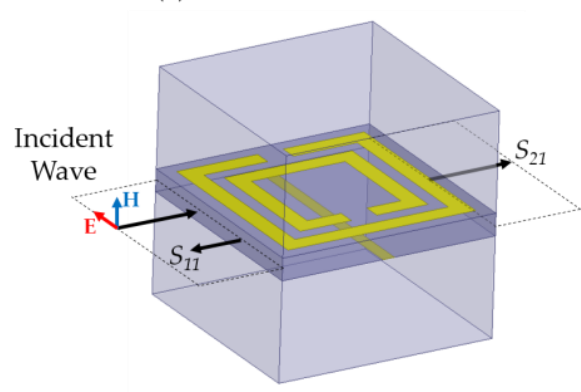

(b) - Homogenized Medium

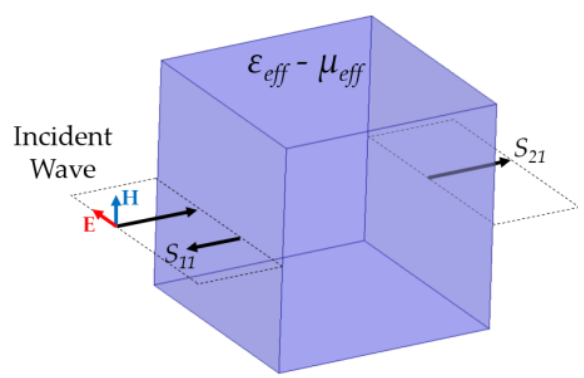

Figure 1.5. Metamaterials homogenization. The metamaterial cell (a) has the same reflection $\left(S_{11}\right)$ and transmission $\left(S_{21}\right)$ coefficients as its homogenised medium (b). 
Lorentz dispersive models, allowing it to accurately simulate such media even with twodimensional FDTD methods and therefore reducing the computational cost. More details on this procedure are described in Appendix A. Although simple to implement, this procedure only handles isotropic metamaterials. A leap forward in the modeling of such media involves the extraction anisotropic parameters, such as those occurring in HMMs, as described next.

\subsubsection{Homogenization of hyperbolic metamaterials}

As previously mentioned, HMMs can be obtained either from wire media [38], [94]-[96] or from planar stacks of alternating metallic and dielectric thin layers [37], [38], [40], [97][100]. There are several methods in the literature capable of extracting the effective parameters of both approaches [74], [75], [84], [85], [76]-[83]. However, planar stacks are usually preferred because their dispersion profiles can be easily tuned either by changing the materials and thicknesses or the operating wavelength [37]. For this reason, this thesis will focus only on layered media.

The simplest homogenization approach, particularly for layered medium, is to utilize the effective medium theory (EMT), proposed by S. M. Rytov in 1956 [83]. Considering a stack of alternating metallic (with permittivity $\varepsilon_{m}$ ) and dielectric (with permittivity $\varepsilon_{d}$ ) layers with thicknesses $l$ and $d$, respectively, the effective perpendicular $\left(\varepsilon_{\perp}\right)$ and parallel $\left(\varepsilon_{\|}\right)$ permittivities are given by [83]:

$$
\begin{gathered}
\varepsilon_{\perp}=\frac{\varepsilon_{m} \varepsilon_{d}}{f f \varepsilon_{d}+(1-f f) \varepsilon_{m}}, \\
\varepsilon_{\|}=f f \varepsilon_{m}+(1-f f) \varepsilon_{d},
\end{gathered}
$$

where $f f$ is the filling factor given by $f f=l /(l+d)$. In this approach, it is assumed that no magnetic response is present on the homogenized media $(\mu=1)$. According to [83], (1.3) and (1.4) are only valid if the layers thickness is much smaller than the operating wavelength $\left(d, l<\lambda_{0} / 10\right)$, which cannot be satisfied in some practical situations. In this sense, a comprehensive parameter retrieval approach in which the thicknesses are taken into account has recently been proposed by Papadakis et al. [81]. The authors also observed that the metal/dielectric stack has magnetic responses when the layers thicknesses are increased, resulting in the perpendicular $\left(\mu_{\perp}\right)$ and parallel $\left(\mu_{\|}\right)$ components of the permeability tensor. Unfortunately, this approach assumes that the stack exhibits no spatial dispersion (with spatial dispersion being defined as the phenomenon whereby the permittivity and/or permeability tensors of the medium depend on the wavevector $\mathbf{k}$ [81]). Chebykin et al. [78] have shown that when $k>>k_{0}$, the wavelength $(\lambda)$ inside the HMM becomes comparable or larger than $d$ and $l$, therefore losing the requirements for a good homogenization $\left(d, l<\lambda_{0} / 10\right)$. Therefore, spatial dispersion becomes an issue that can no longer be neglected at this condition. The importance of taking spatial dispersion into account can be fully understood if, for instance, the enhancement of the spontaneous emission rate $(\Gamma)$ of quantum emitters (QEs) is of interest [37], [40], [55]-[58]. The QE emission can be decomposed into a 
(a) - Layered Medium

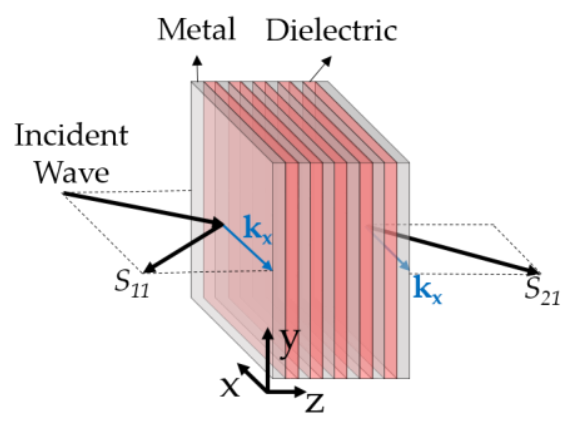

(b) - Homogeneous Medium

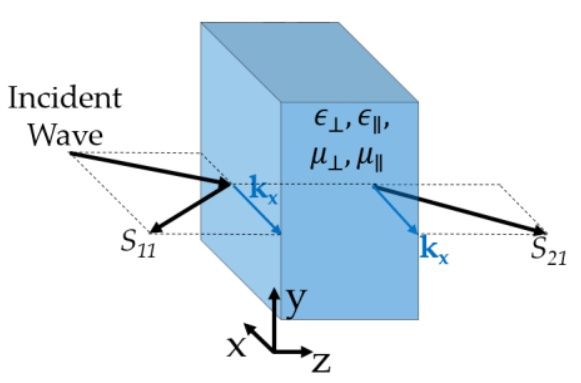

Figure 1.6. Homogenization procedure of a layered medium (a). The layered medium (a) has the same reflection $\left(S_{11}\right)$ and transmission $\left(S_{21}\right)$ parameters as its homogenised medium (b) for each $k x$.

superposition of propagating $\left(k<k_{0}\right)$ and evanescent $\left(k>k_{0}\right)$ waves. Hence, when placed close to the HMM surface, the evanescent field emitted by the QE excites the HMM high$k$ modes. Consequently, an accurate calculation of $\Gamma$ requires the knowledge of the stack effective parameters for all values of $\mathbf{k}\left(\epsilon_{\perp}(\mathbf{k}), \epsilon_{\|}(\mathbf{k}), \mu_{\perp}(\mathbf{k})\right.$ and $\left.\mu_{\|}(\mathbf{k})\right)$, including those whose moduli are much greater than $k_{0}$.

In this thesis we address this problem by proposing a new parameter retrieval approach in which all electromagnetic parameters of the medium are obtained taking spatial dispersion into account [101], described in detail in chapter 2. As in [78], the constraint on nonmagnetic metal/dielectric metamaterials is also relaxed. In our approach, we assume that for each $k_{x}$ value, the metal/dielectric stack of Figure 1.6 (a) can be equivalently represented by the homogeneous, uniaxial anisotropic medium shown in Figure 1.6 (b), with both media expected to produce the same scattering parameters (S-parameters). We also show that a large $\left|k_{x} / k_{0}\right|$ causes the dispersion curve to flatten out (with a corresponding large imaginary part being present) as a result of the absence of propagating modes inside the metamaterial. This behavior is a direct manifestation of spatial dispersion, and if it is not properly considered, $\Gamma$ can be overestimated. In real HMMs, the high- $k$ values induce spatial dispersion and create an upper cut-off for $k$ that limits the number of possible high- $k$ modes [101], and consequently the PDoS.

Next, we discuss one of the main applications for such media, namely, the use of the large PDoS to manipulate the radiation parameters of QEs.

\subsubsection{Manipulating radiation parameters of QE}

The most outstanding feature of HMMs is their large PDoS resulting from the large number of supported high- $k$ modes. Assessing the full range of PDoS is important because the radiation parameters of QEs can be decomposed into a superposition of propagating $\left(k<k_{0}\right)$ and evanescent $\left(k>k_{0}\right)$ waves. Thus, with a proper manipulation of the HMMs PDoS it is possible to control the photon emission of these sources. Intensive 
(a)

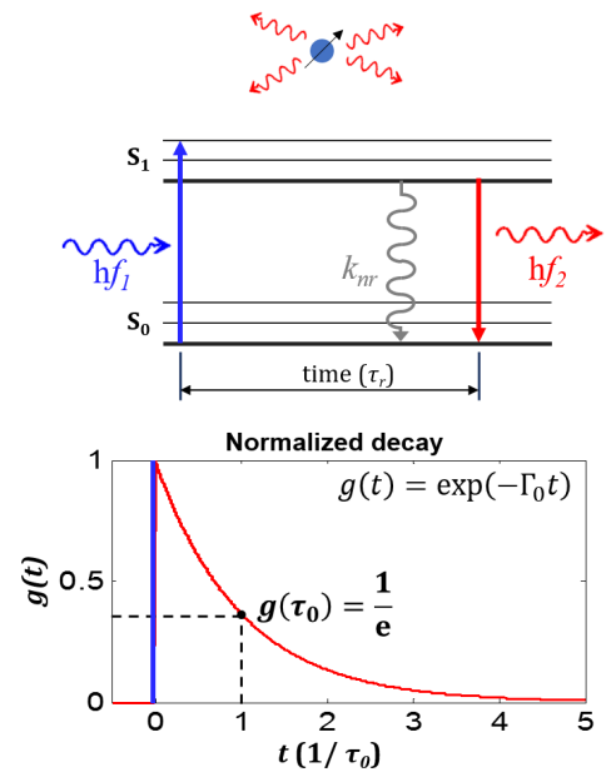

(b)
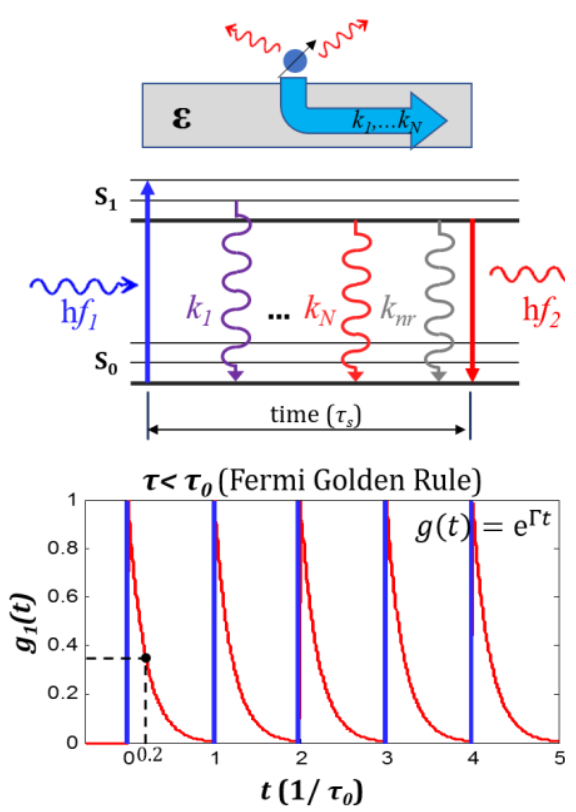

Figure 1.7. Quantum emitters (top) in (a) free space and (b) close to a structure supporting a large number of modes, and their respective energy level diagrams (middle, showing excitation and decay routes) and normalized decay curves (bottom). In (a), a photon with energy $E_{1}=h f_{1}$ excites an electron of the highest occupied molecular orbital (So) to the lowest unoccupied molecular orbital $\left(\mathrm{S}_{1}\right) .\left(k_{1}, \ldots, k_{N}, k_{n r}, h f_{2}\right)$ and $\left(k_{n r}, h f_{2}\right)$ represent the decay routes for a $\mathrm{QE}$ in scenario $(\mathrm{a})$ and $(b)$, respectively. $\left(\Gamma_{0}, \tau_{0}\right)$ and $(\Gamma, \tau)$ are the spontaneous emission rate and the QE lifetime in scenario (a) and (b), respectively.

research has been focused on the study of new approaches to efficiently extract photons from QEs, targeting applications as diverse as lasers [102], LEDs [103], [104], quantum sensors [105], quantum communications systems [106], [107] and single-photon-sources [108]. In order to understand the HMM's role in controlling the emission of $\mathrm{QE}$, we first need to understand the basic photon extraction mechanism of QEs.

Quantum emitters can be atoms, molecules, nanoparticles, quantum dots, quantum wells, or any "quantum confined system" capable of emitting photons [109]. In this dissertation, QEs are considered infinitesimal dipoles governed by the classical electromagnetic equations [109]. The mechanisms used by the HMMs to control the radiation of any QEs are similar. Thus, understanding the mechanism for one type of source is enough to understand the mechanism for any $\mathrm{QE}$. In this sense, imagine a $\mathrm{QE}$ in free space, as shown in Figure 1.7 (a, top). In this system, a photon with energy $E_{1}=h f_{1}(h$ is the Plank constant and $f_{1}$ is the excitation frequency) excites an electron of the highest occupied molecular orbital $\left(\mathrm{S}_{0}\right)$ to the lowest unoccupied molecular orbital $\left(\mathrm{S}_{1}\right)$. The electron remains at $\mathrm{S}_{1}$ for a random period $\left(\tau_{r}\right)$ until it decays back to $S_{0}$, either non-radiatively $\left(k_{n r}\right)$ due to internal 
vibrations or by emitting a photon with energy $E_{2}=h f_{2}$ ( $f_{2}$ is the radiation frequency), as shown in Figure 1.7 (a, middle). For energy conservation, the frequency of the emitted photon must be lower than the excitation photon frequency $\left(f_{1}>f_{2}\right)$, and this frequency displacement is called Stokes shift [109]. One of the most important aspects of this process is the decay time $\tau_{r}$. One approach to quantify the average time required for the electron to decay is to excite a large number of QEs at the same time and, at a large distance (larger than $10 \lambda_{0}$ ), count the photons radiated by the QEs as function of time. The curve obtained with this approach has a negative exponential shape and is defined as the decay curve [109]. If normalized by its maximum, the new decay curve $g(t)$ describes the proportion of QEs that have not radiated a photon at a given time $t$, as seen in Figure 1.7 (a, bottom). Based on $g(t)$, we can define the lifetime of the QE as the time $\tau_{0}$ required for 1-1/e of all QEs to decay, i.e., $\mathrm{g}\left(\tau_{0}\right)=1 /$ e. Furthermore, we can define the spontaneous emission rate $(\Gamma)$ as the proportion of QEs that radiate per unit of time, i.e., $\Gamma=1 / \tau_{0}$. These definitions can also be seen in Figure 1.7 (a, bottom). Therefore, we can write $g(t)$ as:

$$
g(t)=\mathrm{e}^{-\Gamma_{0} t}=\mathrm{e}^{-t / \tau_{0}}
$$

The decay behavior of the QE directly impacts its performance in telecommunication applications. That is, the longer $\tau_{0}$ is, the lower the number of QEs excitations per second, resulting in slower modulation speeds [104] .Therefore, it is crucial to create mechanisms to reduce $\tau_{0}$. One approach is to place the QE close to structures that support a large number of modes, as shown in Figure 1.7 (b, top). These modes create additional 'routes' for the electron to decay from $S_{1}$ to $S_{0}$, as shown in Figure 1.7 (b, middle). According to Fermi's golden rule [110], increasing the number of routes decrease the time the electron remains at $S_{1}$ before decaying to $S_{0}\left(\tau_{s}\right)$. Consequently, the QE lifetime in the proposed situation $\left(\tau_{s}\right)$ is reduced $\left(\tau_{s}<\tau_{0}\right)$ while the spontaneous emission rate $\left(\Gamma_{s}\right)$ is enhanced $\left(\Gamma_{s}\right.$ $\left.>\Gamma_{0}\right)$. Figure 1.7 (b, bottom) shows an example of the normalized decay curve if $\Gamma$ is increased by a factor of 5 . Note that in this situation, the QEs can be excited 5 times faster than if placed in free space (Figure 1.7 (a, bottom)). Thus, the total dissipated power $\left(W_{s}\right)$ is also increased by a factor of 5 when compared to the power the QE would radiate in free space $\left(W_{0}\right)$. In fact, the enhancement factor of the spontaneous emission or power is known as the Purcell factor $(P)[111]$,

$$
P=\frac{\Gamma_{s}}{\Gamma_{0}}=\frac{W_{s}}{W_{0}} .
$$

Nonetheless, not all the energy provided by electrons decaying from $S_{1}$ to $S_{0}$ are converted into radiating photons, since in this scenario part of the energy resulting from this decay is coupled into modes of the structure. Consequently, from the total power dissipated by the dipole, only part is converted into free space radiation $\left(W_{r a d}\right)$ and can be measured at the far field. The ratio between $W_{\text {rad }}$ and $W$ is defined as the QE external quantum efficiency $(\eta),[111]$,

$$
\eta=\frac{W_{r a d}}{W}=\frac{W_{r a d}}{W_{0}} \frac{1}{P} .
$$


Note from (1.7) that in order to increase $P$ and maintain a high $\eta$, it is necessary to increase $W_{\text {rad. }}$ There are several methods aiming at designing structures capable of increasing $P$, such as photonic crystal cavities [112], [113], dielectric nanoholes [114], nano-antennas [115], [116], and HMMs [37], [38]. The use of HMMs is particularly interesting because of their simplicity and broadband characteristics [37], [38]. Remarkably, it has been shown that the QE's lifetime can be decreased by a factor of 100 using HMM [38]. Furthermore, the use of HMMs for increasing the Purcell factor of QE has been experimentally supported in the literature [37]-[40], [97]-[100]. However, these researchers focused only on decreasing $\tau$, without investigation its influence on $\eta$.

In this thesis, we study the influence of the HMM on the QE's $P$ and $\eta$. For practical reasons (that will be explained later on in this thesis), we target the fluorescent molecule Cyanine 7 (Cy7), which is a well-known fluorescent molecule mainly used for medical applications [117]-[119] with emission at $773 \mathrm{~nm}$. We show that although the HMM is capable of increasing $P$ by a factor greater than $100, \eta$ in contrast can be much lower than $1 \%$. Furthermore, as the QE is moved farther from the HMM surface, $P$ decreases and $\eta$ increases. As result, the QEs with low $P$ can have a higher contribution to $W_{\text {rad, }}$, therefore reducing the system's effective Purcell factor $\left(P_{\text {eff }}\right)$.

The low $\eta$ is a consequence of $W$ being mostly coupled into the HMM as lossy high- $k$ modes, preventing them from propagating into free space [37], [38], [40], [97]-[99]. Consequently, the energy of these modes is mostly thermally dissipated inside the HMM, reducing the QE's $\eta$. Aiming at increasing $\eta$, some authors have resorted to nanopatterned HMMs (NPHM) to convert high- $k$ modes into free space modes $\left(k \leq k_{0}\right)$, [33], [48][50], [109]-[118], as seen in the next sub-session.

\subsubsection{Grating-assisted radiation emission of quantum emitters}

The use of diffraction gratings considerably increases the power emitted by the QEs as propagating waves while still maintaining a high Purcell factor. Several types of gratings and nano-patterned structures have been used to increase $\eta$. For instance, in [55], [103], [125]-[127] the authors have used a patterned HMM surrounded by QEs (as shown in Figure 1.8 (a)), while in [128], [129] the QEs are used inside the patterned HMM (Figure 1.8 (b)). Metallic or dielectric gratings on top of the HMM have also been used for this purpose, such as in [124] where the QEs are used at the bottom of the HMM (Figure 1.8 (c)), or in [57], [120], [121] where they are used inside the HMM (Figure 1.8 (d)). Some aspects of each system are outlined as follows:

- $\quad$ Figure 1.8 (a): in this design, the HMM is first patterned and then a polymer film embedded with QEs is deposited on top of the structure. The excitation of the QEs is performed from the top layer. Regarding the QEs properties, $P$ decreases as the QE is moved away from the HMM surface while $W_{\text {rad }}$ does not change considerable. As explained earlier, this causes a reduction in the system's effective $P$. 
(a)

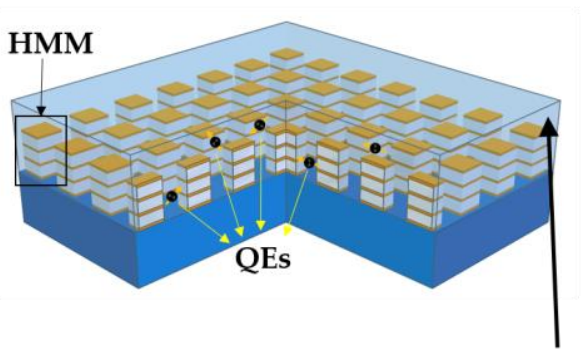

Layer embedded

(c)

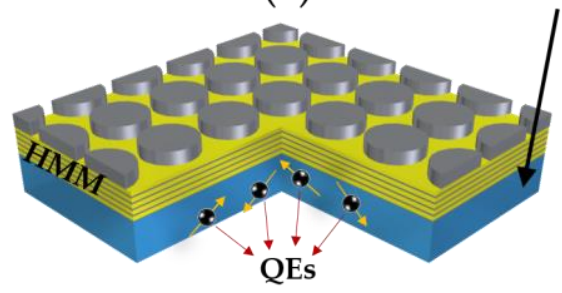

(b)

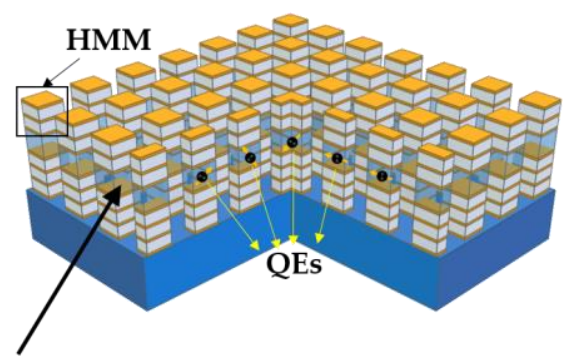

(d)

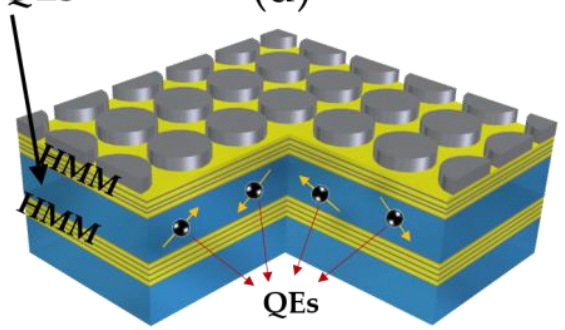

Figure 1.8. (a) Nano-patterned HMM surrounded by QEs [55], [103], [125]-[127]. (b) QEs inside the patterned HMM [128], [129]. (c) Grating on top of the HMM with the QEs embedded in the bottom layers [124]. (d) QEs inside the HMM [57], [120], [121].

- $\quad$ Figure 1.8 (b): differently from (a), the layers containing the QEs are deposited in the middle of the HMM. Furthermore, the layer embedded with QEs is also patterned in the same manner as the HMM. The excitation of the QEs can be performed from the top or bottom layer, but the power required is higher when compared to the system shown in (a). Regarding the QEs properties, $P$ is high and almost constant inside the film since they are bounded by HMMs. However, $W_{\text {rad }}$ is very low in this scenario because light needs to get through the HMM. Consequently, the system's effective $P$ is high but $\eta$ is very low.

- Figure 1.8 (c): in this design, the HMM with the gratings on its top is fabricated over a polymer embedded with QEs. The excitation of the QEs is performed from the substrate and requires low power. Regarding the QEs properties, $P$ decreases exponentially as QE is moved far from the HMM surface, which is due to the low power of evanescent fields that couple into the HMM high- $k$ modes. This also results in an exponentially decay of $W_{\text {rad }}$ as the gratings convert the high- $k$ modes into free space modes. Consequently, the system's effective $P$ is high and $\eta$ is low.

- $\quad$ Figure $1.8(\mathrm{~d})$ : in this design, the polymer film embedded with QEs is located between the HMM layers with the grating on top of the structure. As in (b), it requires more power to excite the QEs. Regarding the QEs properties, both $P$ 
and $W_{\text {rad }}$ are almost constant in the film since they are bounded by HMMs. Consequently, the system's effective $P$ is high and $\eta$ is higher than that shown in (c). Nevertheless, the fabrication of this structure is more challenging.

As can be seen, all types of nano-patterned HMMs have advantages and disadvantages, and as such they need to be carefully chosen for the required application. Regardless the chosen geometry [33], [48]-[50], [109]-[118], the authors resorted to numerical simulations, such as FDTD and FEM, to optimize the HMM geometrical parameters. Besides being computationally costly, numerical simulations do not provide clear-cut insights on how the gratings affect the QEs' behavior.

In this context, we propose in this thesis a semi-analytical method that can be successfully applied to the design of diffraction gratings aimed at optimizing the conversion of high$k$ modes into propagating modes. In addition, this method directly provides the QE's PDoS, which is a computational costly task if carried out numerically. Both 2D and 3D configurations are examined, with the former being used for the grating optimization procedure due to its lower computational cost. The structure is implemented in the 3D model only after the optimum grating parameters have been obtained. Although the proposed method can be applied to any nano-patterned structure, and, due to its advantages, we assume the one shown in Figure 1.8 (c).

Another advantage of the semi-analytical model is the reduced computational burden to obtain $P$ and $W_{\text {rad }}$ as function of the QEs and grating relative position, highlighting the behavior of each QE to the system. Moreover, this mapping is an important tool for the understanding of the decay behavior of the whole system [55], [114] since in most applications the QEs are randomly positioned inside the polymer [55], [57], [103], [120], [121], [125]-[129]. Many authors have treated the decay behavior by fitting $g(t)$ as a sum of exponentials [121], [126], [130], which does not accurately represent the overall behavior of the system. Therefore, to the best of our knowledge, a semi-analytical method that precisely calculates $g(t)$ of a uniform distribution of QEs is a welcome tool to the scientific community and is fully addressed in this thesis.

There have been prior generic semi-analytical techniques proposed to calculate the electric field radiated by dipoles inside anisotropic stratified media [131], [132], nevertheless, as far as the authors are aware, the approach shown in this thesis is the first semi-analytical procedure tailored for accurate calculation and optimization of the emission pattern and the Purcell factor of QEs under a nano-patterned structure, such as grating assisted HMMs. This method, along with its main results, has been published in [124].

Despite being the first semi-analytical model for the analysis of the quantum emitters radiation emission in grating-assisted HMMs (regardless of the grating geometry), this method can only be applied to systems where the QEs are embedded in the substrate, such as in Figure 1.8 (c) However, in order to cover all situations shown in Figure 1.8, we have improved the proposed method even further, as described in the next sub-section. 


\title{
1.1.4 General approach for calculating the radiation emission of QEs inside bounded hyperbolic metamaterials
}

\begin{abstract}
Aiming at solving all situations depicted in Figure 1.8, we have extended our previous semi-analytical approach to calculate, both in two and three dimensions (2D and 3D), all radiation parameters of a QE embedded in a generalized nano-patterned HMM. This method allows multiple electromagnetic sources arbitrarily distributed in the cover, substrate, and any inner layer to be used simultaneously. The flexibility of placing the source inside any layer (nano-patterned or not) allows us to very efficiently obtain the radiation emission parameters of any previously published HMM/source configuration shown in Figure 1.8. The low computational cost of our approach makes it attractive for optimizing the NPHMs geometrical parameters that maximize $\eta$ for a desired $\Gamma$. Furthermore, we suggest a more realistic procedure to calculate the decay behavior of multiple QEs arbitrarily distributed in the NPHM. This calculation is only possible with the knowledge of $\Gamma$ and $\eta$ mapped for all possible positions of the QEs, which is easily achieved with the proposed model. In addition to increase $\eta$ the time required to build the $\Gamma$ and $\eta$ maps (used in the calculation of the decay behavior) is reduced by approximately $96 \%$ when compared to those numerically calculated via FDTD.
\end{abstract}

\subsection{Objectives of this thesis}

There has been an arduous work over the past few years in searching new approaches to efficiently decrease the lifetime of QEs while maintaining a high quantum efficiency using HMM. However, there are several aspects of this new technologies that are still unsolved as pointed out in previous discussions. In this way, this doctoral thesis contributes to this area by investigating the two following problems: homogenization of HMM with spatial dispersion analysis; characterization of the radiation parameters of QEs inside nanopatterned HMM by means of the development of novel and efficient semi-analytical modelling methods. Henceforth, the original contributions of this thesis are briefly outlined as follows:

I. New parameter retrieval approach in which all electromagnetic parameters of a biaxial anisotropic medium are obtained taking spatial dispersion into account. The constraint on nonmagnetic metal/dielectric hyperbolic metamaterials is also relaxed. We investigate different metal/dielectric stacks and show that all of them have a clear magnetic response, particularly for thicker layers. We also show that a large $\left|k_{x} / k_{0}\right|$ causes the dispersion curve to flatten out (with a corresponding large imaginary part being present) as a result of the absence of propagating modes inside the metamaterial. This behavior is a direct manifestation of spatial dispersion. We show that, if spatial dispersion is not properly taken into account the Purcell factor is overestimated. Our approach shows excellent agreement with the accurate Purcell factor calculation of [109] used as benchmark. 
II. A novel semi-analytical method that can be successfully applied to the design of diffraction gratings aimed at optimizing the conversion of high- $k$ modes into propagating modes. In addition, this method directly provides the QE's PDoS, which is a computationally costly task if carried out numerically. Both $2 \mathrm{D}$ and 3D configurations are examined, with the former being used for the grating optimization procedure due to its lower computational cost. The structure is implemented in the 3D model only after the optimum grating parameters have been obtained. The proposed HMM design assumes that QEs are embedded in the substrate since the amount of power necessary to excite them is reduced in this case [39], [57], [121]-[123], [128], [129]. As a result, the QEs closer to the HMM surface emit more power to the far field, contributing to the decrease of the system's lifetime. In addition, the proposed method facilitates the grating design, which is useful for controlling the QEs' emission directivity. Another advantage of this method is the reduced computational burden to obtain the Purcell factor and the radiated power as function of the QEs and grating relative position, highlighting the behavior of each QE to the system.

III. A generalized semi-analytical model to calculate, both in 2D and 3D, all radiation parameters of any source (including QEs modelled as dipoles) embedded in any layer of a nano-patterned stratified medium (NSM). Moreover, this method also allows multiple electromagnetic sources arbitrarily distributed in the cover, substrate, and any inner layer to be used simultaneously. The flexibility of placing the source inside any layer (nanopatterned or not) allows us to obtain the radiation emission parameters for all situations depicted in Figure 1.8. The radiation parameters are mapped here as function of the QE and NPHM relative position, which is facilitated by the method's low computational cost.

IV. A new semi-analytical method to precisely calculate $g(t)$ of a uniform distribution of QEs. This approach is only possible with the knowledge of $P$ and $W_{\text {rad }}$ as function of the QEs and grating relative position, which are easily obtained from both semi-analytical models proposed in this thesis. The analytically calculated $g(t)$ allows the system's effective quantum efficiency $\left(\eta_{\text {eff }}\right)$ and emission rate $\left(\Gamma_{\text {eff }}\right)$ to be directly obtained assuming multiple arbitrarily distributed electromagnetic sources inside the NPHM. In this sense, we also propose here a new procedure to optimize the NPHM geometrical parameters to maximize $\eta_{\text {eff }}$ while achieving the desired $\Gamma_{\text {eff. }}$.

\subsection{Organization of the Thesis}

This thesis is organized as follows:

Chapter 2 provides the method to retrieve the electromagnetic parameters of biaxial anisotropic metamaterials considering spatial dispersion. The chapter lays out the 
mathematical formalism of the proposed parameter retrieval approach. Then we analyze different metal/dielectric stacks as a function of the metal thickness and fill factor. We also discuss the role of the number of layers on the performance of the parameter retrieval approach. Later, we deal with the calculation of the Purcell factor, showing a comparison between the results obtained both with the EMT and with our approach. Finally, we present some concluding remarks about the homogenization procedure.

Chapter 3 presents the main design concepts of HMMs and the mathematical formalism to calculate $P$ and $\eta$ of a QE embedded in a bounded media. Then the proposed model is applied to the analysis of the QE electromagnetic behavior inside the host layer. In sequence, a uniform distribution of QEs is assumed inside the host layer so that the mathematical formalism for the computation of $g(t)$ can be obtained. Finally we describe the influence of the host film thickness on $g(t)$.

Chapter 4 introduces the semi-analytical formalism to calculate in 2D and 3D the radiation parameters of QEs placed close to nano-patterned structure. The chapter begins presenting the mathematical formalism. Next, we apply our optimization procedure to a metallic grating placed on top of an alternating $\mathrm{Ag}$ and titanium dioxide $\mathrm{TiO}_{2}$ layers. We begin the analysis with the $2 \mathrm{D}$ optimization of the grating structure, followed by the 3D simulation carried out with the optimized parameters. The figures-of-merit are the radiation pattern and the Purcell factor, both as function of the QE position. Finally, we present some concluding remarks about the semi analytical model.

Chapter 5 presents a general semi-analytical model to calculate, both in two and three dimensions (2D and 3D), all radiation parameters of any electromagnetic source type (including QEs modeled as dipole) embedded in any layer of a NSM. Moreover, this model also allows two additional sources, one at the top and other at the bottom layer, to be used simultaneously. The flexibility of placing the source in any layer, nano-patterned or not, allows us to calculate the radiation emission parameters of any previously published HMM/source configuration shown in Figure 1.8.

Chapter 6 presents the conclusion remarks highlighting the original contributions of this thesis to the metamaterials scientific community. Furthermore, we also show the future perspectives to be explore by the PhD candidate during his post-doc. 



\section{Chapter 2}

\section{Homogenization of}

uniaxial anisotropic metamaterials 


\section{Chapter 2 - Summary}

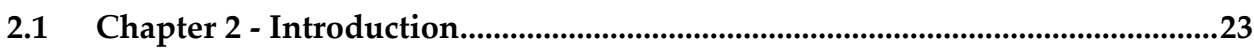

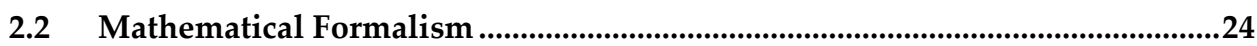

2.2.1 S-Parameters of the Metal-Dielectric stack ........................................................24

2.2.2 S-Parameters of the Anisotropic Medium .....................................................26

2.2.3 Extraction of the Constitutive Parameters from the S-Parameters of the Slab

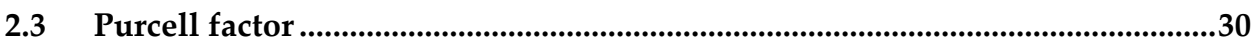

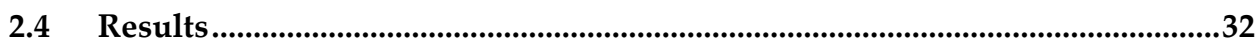

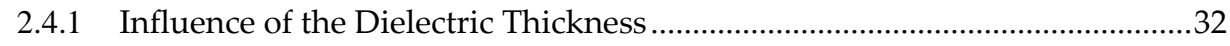

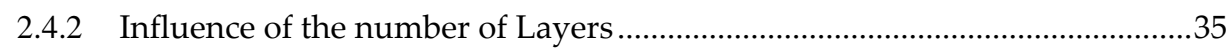

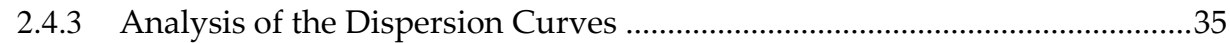

2.5 Calculation of the Purcell Factor .............................................................................41

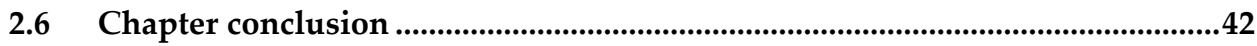




\subsection{Chapter 2 - Introduction}

In this chapter, we propose a new parameter retrieval approach in which all electromagnetic parameters of the medium are obtained taking spatial dispersion into account. This approach is from hereon named a complete parameter retrieval approach, or CPR. As in [78], the constraint on nonmagnetic metal/dielectric metamaterials is also relaxed. We investigate different metal/dielectric stacks and show that all of them have a clear magnetic response, particularly for thicker layers. We also show that a large $\left|k_{x} / k_{0}\right|$ causes the dispersion curve to flatten out (with a corresponding large imaginary part being present) as a result of the absence of propagating modes inside the metamaterial. This behavior is a direct manifestation of spatial dispersion. The impacts of the spatial dispersion in the calculation of the Purcell factor of Rb atoms $10 \mathrm{~nm}$ above the HMM surface for the emission wavelengths of $422 \mathrm{~nm}$ and $780 \mathrm{~nm}$ (Rb emission wavelengths) is also investigated. The results are compared with three different approaches, namely, the accurate result considering the actual non-homogenized geometry, the approach in [15], and the EMT approach. We show that if spatial dispersion is not properly taken into account the Purcell factor is overestimated. In contrast the CPR approach shows excellent agreement with the accurate Purcell factor.

This chapter is organized as follows: In Section 2.1 and 2.2 we lay out the mathematical formalism of the proposed parameter retrieval approach and how to calculate the Purcell factor, respectively. In Section 2.3 we analyze different metal/dielectric stacks as a function of the metal thickness and fill factor. We also discuss the role of the number of layers on the performance of the parameter retrieval approach. Section 2.4 deals with the calculation of the Purcell factor, showing a comparison between the results obtained both with the EMT and with the CPR approach. Finally, Section 2.5 presents some concluding remarks

Part of the work presented in this chapter has been published as:

A. F. Mota, A. Martins, J. Weiner, F. L. Teixeira and B.-H. V. Borges, "Constitutive parameter retrieval for uniaxial metamaterials with spatial dispersion," Physical Review B, vol. 94, 115410, 2016. The copyrights permission is found in Annex 1 of this thesis. 


\subsection{Mathematical Formalism}

The CPR approach assumes that the metal/dielectric stack of Figure 2.1 can be equivalently represented by the homogeneous, uniaxial anisotropic medium shown in Figure 2.2, with both media expected to produce the same scattering parameters (Sparameters). The present approach works as follows: we first calculate the S-parameters for both structures in Figure 2.1 and Figure 2.2 (see subsections 2.2.1 and 2.2.2), and then we combine the corresponding equations (subsection 2.2.3) so that expressions for the permittivity and permeability tensor components of the homogenous medium can be obtained. The procedure is carried out for both TE and TM polarizations simultaneously, since the extracted parameters are assumed to be the same for both polarizations. This allows us to relax the constraint on nonmagnetic metal/dielectric metamaterial.

\subsubsection{S-Parameters of the Metal-Dielectric stack}

The extraction procedure begins with the calculation of the S-parameters for both polarization, TE $\left(S_{11}^{s}, S_{21}^{s}\right)$ and TM $\left(S_{11}^{p}, S_{21}^{p}\right)$ of the metal-dielectric stack with dielectric thickness $d$ and metal thickness $l$ (Figure 2.1). The CPR approach assumes the stack as symmetric so that $S_{11}=S_{22}$ and $S_{21}=S_{12}$. As a result, and in order to keep the fill factor $f f=l /(l+d)$ unchanged, the thickness of the first and last layers of the stack must be half the metal thickness $(l / 2)$. Considering a stack of $N$ layers, with metal and dielectric relative permittivities $\varepsilon_{m}$ and $\varepsilon_{d}$, respectively, the S-parameters are obtained as follows [133]:

$$
\left[\begin{array}{c}
1 \\
S_{11}^{\chi}
\end{array}\right]=\left(D_{0}^{\chi}\right)^{-1} T^{\chi} D_{N+1}^{\chi}\left[\begin{array}{c}
S_{21}^{\chi} \\
0
\end{array}\right]
$$

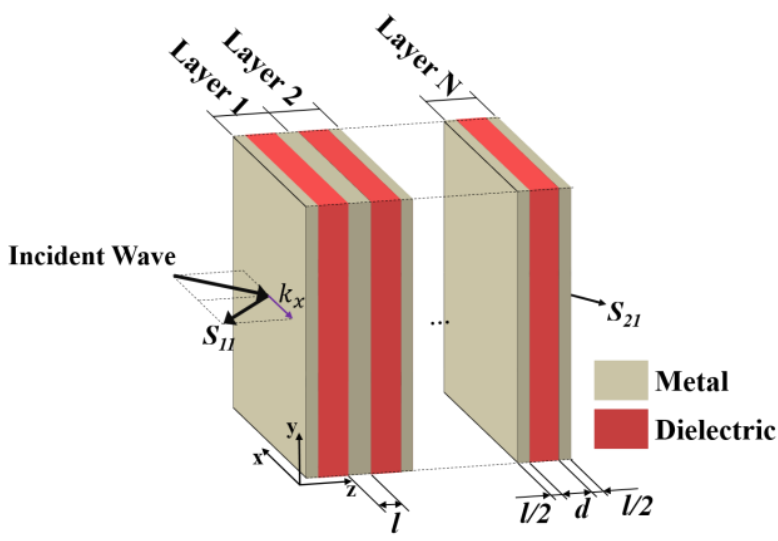

Figure 2.1. Metal/dielectric stack with incident wave impinging on the surface from the left. Part of the incident wave is reflected $\left(S_{11}\right)$ and part is transmitted $\left(S_{21}\right)$. The metal and dielectric thicknesses are 1 and $d$, respectively. 


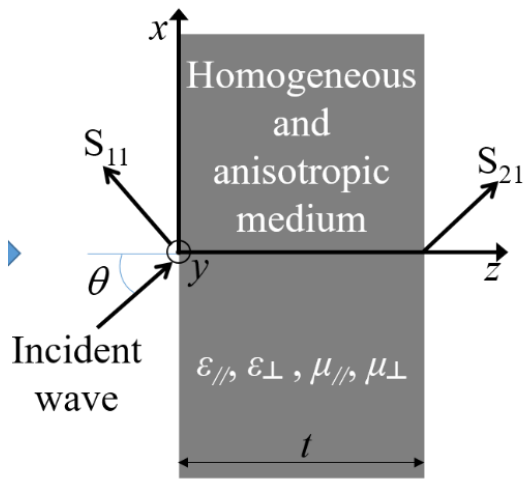

Figure 2.2. Equivalent homogenous anisotropic medium.

where the index $\chi$ are equal to $s$ or $p$ for transversal electric (TE) or magnetic (TM) polarization, and

$$
\begin{gathered}
T^{\chi}=\prod_{i=1}^{N} D_{i}^{\chi} P_{i}\left(D_{i}^{\chi}\right)^{-1}, \\
D_{i}^{s}=\left[\begin{array}{cc}
1 & 1 \\
\frac{k_{z}^{i}}{\varepsilon_{i}} & -\frac{k_{z}^{i}}{\varepsilon_{i}}
\end{array}\right], \\
D_{i}^{p}=\left[\begin{array}{cc}
1 & 1 \\
k_{z}^{i} & -k_{z}^{i}
\end{array}\right], \\
P_{i}=\left[\begin{array}{cc}
\mathrm{e}^{-\mathrm{j} k_{z}^{l} d_{i}} & 1 \\
1 & \mathrm{e}^{\mathrm{j} k_{z}^{l} d_{i}}
\end{array}\right],
\end{gathered}
$$

with $\varepsilon_{l}=\varepsilon_{m}$ (for $i$ odd) or $\varepsilon_{l}=\varepsilon_{d}$ (for $i$ even), $k_{z}^{i}$ is the wavevector in the $z$ direction inside the $i$ th layer, and $d_{i}$ is the thickness of the $l$ th layer (with $d_{1}=d_{N}=l / 2$ ).

The parameter extraction is performed as a function of the wavevector in the $x$ direction $\left(k_{x}\right)$ in order to visualize the effects of the spatial dispersion in the HMM. This choice is justified since the boundary conditions for $k_{x}$ require this wavevector to be the same (phase matching) in all layers of the stack (Figure 2.1) and inside the anisotropic metamaterial (Figure 2.2). As such, $k_{z}^{i}$ can be calculated from the following relation

$$
k_{z}^{i}=\sqrt{k_{0}^{2} \varepsilon_{i}-k_{x}^{2}}
$$




\subsubsection{S-Parameters of the Anisotropic Medium}

The next step consists in calculating the S-parameters of the metal/dielectric stack, treated here as a homogeneous and anisotropic slab with thickness $t$ (Figure 2.2). The relative permittivity and permeability tensors of an anisotropic media can be written as $\boldsymbol{\epsilon}=$ $\operatorname{diag}\left[\varepsilon_{x}, \varepsilon_{y}, \varepsilon_{z}\right]$ and $\boldsymbol{\mu}=\operatorname{diag}\left[\mu_{x}, \mu_{y}, \mu_{z}\right]$, respectively. Due to the symmetry of the stack (Figure 2.1), it follows that $\varepsilon_{x}=\varepsilon_{y}=\varepsilon_{\perp}, \varepsilon_{z}=\varepsilon / \prime, \mu_{x}=\mu_{y}=\mu_{\perp}$ and $\mu_{z}=\mu / /$. We start with the wave equations for Electric (E) and Magnetic $(\mathbf{H})$ fields which are written, respectively, as

$$
\begin{gathered}
\mathbf{k} \times\left\{\boldsymbol{\mu}(\mathbf{k}, \omega)^{-1} \cdot[\mathbf{k} \times \mathbf{E}(\mathbf{k}, \omega)]\right\}+k_{0}^{2} \varepsilon(\mathbf{k}, \omega) \cdot \mathbf{E}(\mathbf{k}, \omega)=0, \\
\mathbf{k} \times\left\{\boldsymbol{\varepsilon}(\mathbf{k}, \omega)^{-1} \cdot[\mathbf{k} \times \mathbf{H}(\mathbf{k}, \omega)]\right\}+k_{0}^{2} \boldsymbol{\mu}(\mathbf{k}, \omega) \cdot \mathbf{H}(\mathbf{k}, \omega)=0 .
\end{gathered}
$$

In the remaining equations the $(\mathbf{k}, \omega)$ dependence is omitted for the sake of simplicity. Since the anisotropic medium is uniaxial, the TE $\left(E_{y}, H_{x}, H_{z}\right)$ and TM $\left(H_{y}, E_{x}, E_{z}\right)$ solutions can be decoupled. Therefore, the solutions of (2.7) and (2.8) for TE and TM polarizations are, respectively

$$
\begin{gathered}
E_{y}=E_{0} \mathrm{e}^{\mathrm{j}\left(\beta_{z}^{s} z+\beta_{x}^{s} x\right)}, \\
H_{y}=H_{0} \mathrm{e}^{\mathrm{j}\left(\beta_{z}^{s} z+\beta_{x}^{s} x\right)}
\end{gathered}
$$

where $E_{0}$ and $H_{0}$ are the amplitudes of the electric (TE) and magnetic (TM) fields; $\beta_{z}^{T E}$, $\beta_{x}^{T E}, \beta_{z}^{T M}$, and $\beta_{x}^{T M}$ are the $\mathrm{z}$ and $\mathrm{x}$ components of the wavevector. After substituting (2.9)

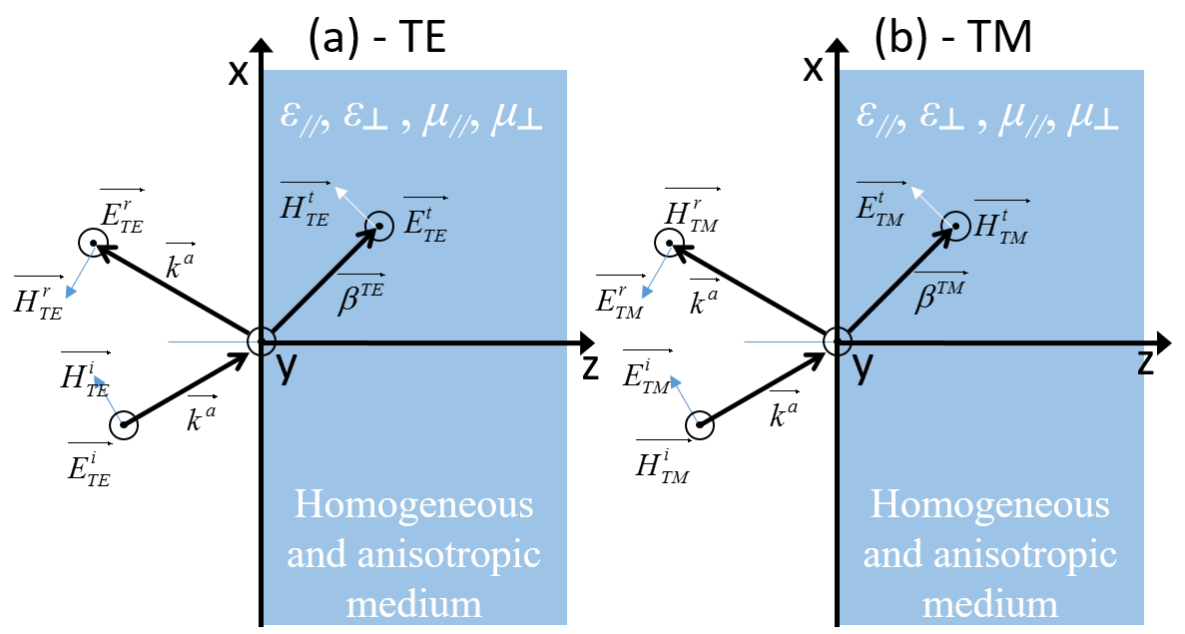

Figure 2.3. Diagrams show field components incident on the interface between air and a homogenous, anisotropic medium for (a) TE and (b) TM polarizations 
into (2.7) and (2.10) into (2.8), the dispersion relations for TE and TM modes, respectively, become

$$
\begin{aligned}
& \frac{\left(\beta_{z}^{s}\right)^{2}}{\mu_{\perp}}+\frac{\left(\beta_{x}^{s}\right)^{2}}{\mu_{\|}}=k_{0}^{2} \varepsilon_{\perp}, \\
& \frac{\left(\beta_{z}^{s}\right)^{2}}{\varepsilon_{\perp}}+\frac{\left(\beta_{x}^{s}\right)^{2}}{\varepsilon_{\|}}=k_{0}^{2} \mu_{\perp},
\end{aligned}
$$

We should note that spatially dispersive media often necessitate additional boundary conditions (ABCs) according to the specific type of spatial dispersion model assumed [75], [134]-[139]. In particular, ABCs are invoked in the most common spatial dispersion model, viz. the isotropic ${ }^{1}$ hydrodynamic Drude model [140] and its variants, since this model supports longitudinal modes, which calls for enforcing either the normal component of current density to be zero at the material interface (in models supporting eddy currents [140]), or for enforcing both the normal and tangential current components to be zero at the material interface (in models not supporting eddy currents [141]).

Here, spatial dispersion is treated not from a first-principles hydrodynamic model but instead as a direct consequence of an S-parameter extraction, homogenization, and inversion procedure. In particular, we do not invoke an isotropic spatially dispersive model, but extract optical parameters from a homogenized, highly anisotropic (uniaxial) material by an inversion procedure of S-parameters determined by the application of conventional boundary phase-matching conditions to a periodic stack of alternating metal and dielectric slabs. Optical excitation in a frequency regime far below collective polariton resonances, where the extra transverse and longitudinal wave amplitudes are negligible [134], [135], permit the application of conventional phase-matching conditions at surfaces to determine the effective medium parameters ${ }^{2}$. A similar procedure has been applied previously [142].

Next, we calculate the reflection and transmission coefficients for both TM and TE polarizations as depicted in Figure 2.3 (a) and (b), respectively. In both polarizations, the incident wave travels in air from the left with wavevector components $k_{x}$ and $k_{z}$ (given by (6) with $\varepsilon_{p}=1$ ). The incident wave (superscript $i$ ) gets partially reflected (superscript $r$ ) and partially transmitted (superscript $t$ ) into the anisotropic medium. As a result, after imposing the continuity of tangential components on the interface, the following set of equations is obtained for both TE and TM polarizations

\footnotetext{
${ }^{1}$ Despite being typically represented in tensorial form, the hydrodynamic Drude model is isotropic since the two terms of the tensor expression represent longitudinal and transverse permittivity expression with respect to the propagation vector, and not to any preferential direction of space.

${ }^{2}$ On the subject of $\mathrm{ABCs}$, we should note in passing that $\mathrm{ABC}$ s are also necessitated in some types on anisotropic spatially dispersive media obtained from homogenization procedures such as wire media [75]. However, in such media the required $\mathrm{ABC}$ is again for the normal component of current density to be zero at the metamaterial interface because of the existence of longitudinal current modes along the metal wires. In our problem, the metal layers are transverse, and as such, longitudinal currents modes are not supported, obviating the need for such condition.
} 


$$
\left\{\begin{array}{c}
A_{y}^{i}=A_{0} \mathrm{e}^{\mathrm{j}\left(k_{x} x+k_{z} z\right)} \\
A_{y}^{r}=R_{\chi} A_{0} \mathrm{e}^{\mathrm{j}\left(k_{x} x-k_{z} z\right)} \\
A_{y}^{t}=T_{\chi} A_{0} \mathrm{e}^{\mathrm{j}\left(\beta_{x}^{\chi} x+\beta_{z}^{\chi} z\right)} \\
B_{x}^{i}=-A_{0} \frac{k_{z}}{\omega v_{0}} \mathrm{e}^{\mathrm{j}\left(k_{x} x+k_{z} z\right)} \\
B_{x}^{r}=R_{\chi} A_{0} \frac{k_{z}}{\omega v_{0}} \mathrm{e}^{\mathrm{j}\left(k_{x} x-k_{z} z\right)} \\
B_{x}^{r}=T_{\chi} A_{0} \frac{k_{z}}{\omega v_{0} v_{\perp}} \mathrm{e}^{\mathrm{j}\left(\beta_{x}^{\chi} x+\beta_{z}^{\chi} z\right)}
\end{array}\right.
$$

where $R_{\chi}$ and $T_{\chi}$ are the reflection and transmission coefficients, respectively, $A$ is assumed as $E$ (for TE) or $H$ (for TM), $B$ is assumed as $H$ (for TE) or $-E$ (for TM), and $v$ is assumed as $\mu$ (for TE) or $\varepsilon$ (for TM). The boundary relationships impose the following relation:

$$
k_{x}=\beta_{x}^{s}=\beta_{x}^{p} .
$$

With the help of the boundary relationships for the tangential field components, the following equations for $R_{\chi}$ and $T_{\chi}$ are obtained,

$$
\begin{aligned}
& R_{\chi}=\frac{Z_{\chi}-1}{Z_{\chi}+1}, \\
& T_{\chi}=1-R_{\chi^{\prime}} \\
& Z_{\chi}=\frac{k_{z} v_{\perp}}{\beta_{z}^{\chi}},
\end{aligned}
$$

where $v$ assumes $\mu$ for TE or $\varepsilon$ for TM polarization. Once the transmission and reflection coefficients are calculated, the scattering parameters for the slab of a homogeneous and anisotropic media with thickness $t$ can be obtained as follows [68]:

$$
\begin{aligned}
& S_{11}^{\chi}=\frac{R_{\chi}\left(1-\mathrm{e}^{2 \mathrm{j} \beta_{z}^{\chi} t}\right)}{1-R_{\chi}^{2} \mathrm{e}^{2 \mathrm{j} \beta_{z}^{\chi} t}}, \\
& S_{21}^{\chi}=\frac{\left(1-R_{\chi}^{2}\right) \mathrm{e}^{\mathrm{j} \beta_{z}^{\chi} t}}{1-R_{\chi}^{2} \mathrm{e}^{2 \mathrm{j} \beta_{z}^{\chi} t}},
\end{aligned}
$$




\subsubsection{Extraction of the Constitutive Parameters from the S-Parameters of the Slab}

At this point, both the S-parameters of the stack (Figure 2.1) and of its homogenized slab (Figure 2.2) have been obtained. In order to extract the constitutive parameters, equations (2.18) and (2.19) must be solved for $\beta_{z}^{\Delta}$ and $Z_{\Delta}$ as functions of $S_{11}$ and $S_{21}$. Thus, after inverting (2.18) and (2.19) we get [61],

$$
\begin{gathered}
Z_{\chi}= \pm \sqrt{\frac{\left(1+S_{11}^{\chi}\right)^{2}-\left(S_{21}^{\chi}\right)^{2}}{\left(1-S_{11}^{\chi}\right)^{2}-\left(S_{21}^{\chi}\right)^{2}}} \\
\beta_{z}^{\chi}=\frac{1}{t}\left[\cos ^{-1}\left\{\frac{1}{S_{21}^{\chi}}\left[1-\left(S_{21}^{\chi}\right)^{2}+\left(S_{11}^{\chi}\right)^{2}\right]\right\}+2 \pi n\right],
\end{gathered}
$$

where the sign of (2.20) is chosen so as to satisfy $\operatorname{Re}\left\{Z_{\chi}\right\}>0$, and $n$ is an integer obtained via the phase unwrapping method [63]. Thus $\mu_{\perp}$ and $\varepsilon_{\perp}$ are obtained from (2.17) as follows,

$$
\begin{aligned}
& \mu_{\perp}=\frac{Z_{s} \beta_{z}^{s}}{k_{z}}, \\
& \varepsilon_{\perp}=\frac{Z_{p} \beta_{z}^{p}}{k_{z}} .
\end{aligned}
$$

Finally, using (2.11) and (2.12), the remaining parameters $\mu_{\|}$and $\varepsilon_{\|}$are obtained as follows

$$
\begin{aligned}
& \mu_{\|}=\frac{k_{x}^{2} \mu_{\perp}}{k_{0}^{2} \mu_{\perp} \varepsilon_{\perp}-\left(\beta_{z}^{s}\right)^{2}}, \\
& \varepsilon_{\|}=\frac{k_{x}^{2} \varepsilon_{\perp}}{k_{0}^{2} \mu_{\perp} \varepsilon_{\perp}-\left(\beta_{z}^{p}\right)^{2}} .
\end{aligned}
$$

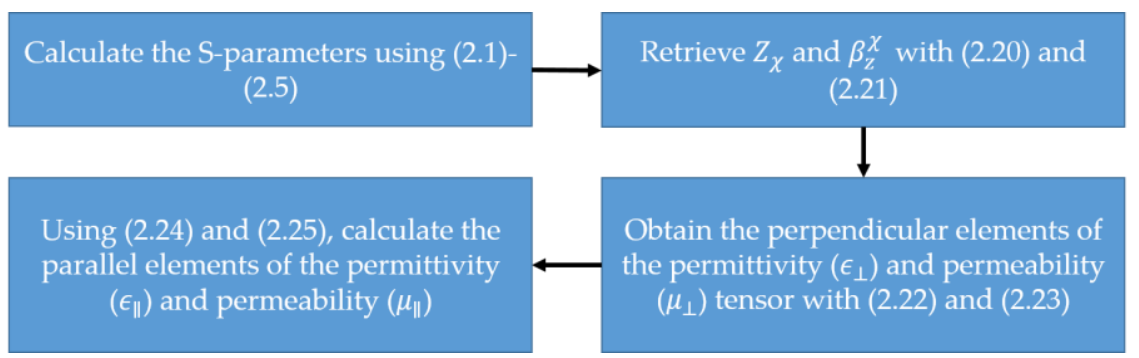

Figure 2.4. Diagram shows the algorithm for extracting constitutive parameters. 
The algorithm for the extraction of the constitutive parameters of a metamaterial is diagrammed in Figure 2.4.

\subsection{Purcell factor}

As mentioned before, the most outstanding feature of hyperbolic metamaterials is the existence of photonic states with wavevector $(k)$ much larger than the free space wavevector $\left(k_{0}\right)$. These high- $k$ modes can be excited, for instance, by placing a dipole close to the HMM surface [143]. Since the dipole radiation is a superposition of propagating $\left(k_{x} \leq k_{0}\right)$ and evanescent $\left(k_{x} \geq k_{0}\right)$ waves, if the dipole is sufficiently close to the surface its evanescent waves will couple to the high- $k$ states of the HMM. This characteristic is extremely important for the enhancement of the Purcell factor [37], [40], [55]-[58]. This approach (using hyperbolic metamaterials) is different from the method normally used in the literature, that involves cavities [144], [145], photonic crystals [146], dielectric nanoholes [114], nano-antennas [115], [116]. In addition [147], the HMM is the only method which allows the enhancement in a broadband [37], [122], [148].

In order to better understand the Purcell factor, imagine a QE excited to a higher energy level, it will eventually decay emitting radiation in a certain wavelength. This radiation can be modulated positioning the atom above a reflecting surface. The surface reflects part of the energy, which can interact either constructively or destructively with the emitted radiation of the QE, depending on the distance of the QE to the surface and also on $k$. This interference creates preferential decay channels ( $k$ values) that may contribute to a higher dissipation power of the atom to these $k$ states [3], [9]-[12].

When the atom is positioned above conventional materials (dielectric or metallic), the number of modes allowed for the atom to decay is reduced. In contrast, HMMs support high- $k$ states that greatly increase the PDoS allowing the atom to decay faster [37], [40], [55]-[58]. To better understand this effect, consider a dipole at a distance $d$ from a surface with reflection coefficient $S_{11}^{p}$ for TM modes and $S_{11}^{s}$ for TE modes, as seen in Figure 2.5.

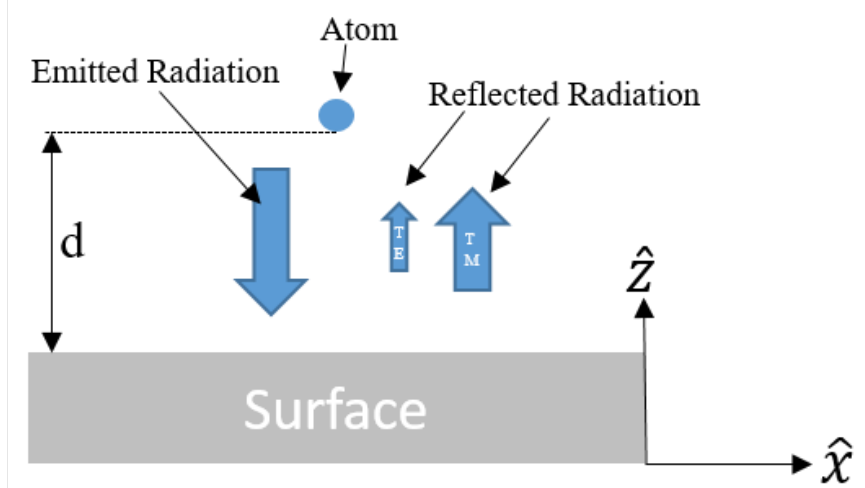

Figure 2.5. Diagram illustrating the radiation of a dipole at a distance $d$ from the surface. Note that the magnitude of the reflected wave is polarization dependent (TE or TM). 
To calculate the QE decay modes, we can use the normalized power dissipated by the dipole as a function of the parallel component of the wavevector $\left(k_{x}\right)$. The QE above the surface are randomly distributed, thus, the electric dipole can be oriented perpendicular ( $\mathrm{z}$-axis) or parallel ( $\mathrm{x}$ - and $\mathrm{y}$-axes) to the HMM surface. Therefore, the normalized dissipated power density (normalized with respect to the power dissipated by a $\mathrm{QE}$ decaying in free space) can be calculated for each orientation as follows [149]:

$$
\begin{gathered}
\frac{\mathrm{d} W_{\perp}}{\mathrm{d} k_{x}}=\frac{3}{2} \frac{1}{k_{z}}\left(\frac{k_{x}}{k_{0}}\right)^{3}\left(1-S_{11}^{p} \mathrm{e}^{2 j k_{z} d}\right), \\
\frac{\mathrm{d} W_{\|}}{\mathrm{d} k_{x}}=\frac{3}{4} \frac{1}{k_{z}} \frac{k_{x}}{k_{0}}\left[1+S_{11}^{s} \mathrm{e}^{2 \mathrm{j} k_{z} d}+\frac{k_{z}^{2}}{k_{0}^{2}}\left(1-S_{11}^{p} \mathrm{e}^{2 \mathrm{j} k_{z} d}\right)\right], \\
\frac{\mathrm{d} W}{\mathrm{~d} k_{x}}=\frac{1}{3} \frac{\mathrm{d} W_{\perp}}{\mathrm{d} k_{x}}+\frac{2}{3} \frac{\mathrm{d} W_{\|}}{\mathrm{d} k_{x}},
\end{gathered}
$$

where $W_{\perp}$ and $W_{\|}$are the dissipated power when the dipole is oriented perpendicular or parallel to the HMM surface, respectively, and $W$ is the total power dissipated by the QE. In [149], the authors show that it is possible to increase the radiation of an QE by a factor of 100 when the atom is positioned $10 \mathrm{~nm}$ above a HMM. The total normalized dissipated power can be calculated via the integration of the normalized dissipated power density. As a result of being normalized with respect to the dissipated power in free space, if $S_{11}^{p}=S_{11}^{s}=0$ (representing free space), the result of the integral will be one. Therefore, assuming the dipole is positioned above the surface, the dissipated power will be equivalent to the relation between the dipole dissipated power to the power it would dissipate if it was in free space. This relation is formally known as the Purcell factor. The Purcell factor $(P)$ is calculated as follows:

$$
\begin{aligned}
P_{\perp} & =\int_{0}^{\infty} \frac{\mathrm{d} W_{\perp}}{\mathrm{d} k_{x}} \mathrm{~d} k_{x} \\
P_{\|} & =\int_{0}^{\infty} \frac{\mathrm{d} W_{\|}}{\mathrm{d} k_{x}} \mathrm{~d} k_{x} \\
P & =\int_{0}^{\infty} \frac{\mathrm{d} W}{\mathrm{~d} k_{x}} \mathrm{~d} k_{x}
\end{aligned}
$$

As can be seen in (2.26)-(2.31), the Purcell factor is highly dependent on the parallel component of the wavevector $\left(k_{x}\right)$, justifying the retrieval parameters proposed in this chapter. Furthermore, an interesting analysis is to separate the contributions of the energy that is radiated to free space from those which are evanescent. The evanescent emission can only propagate in the HMM, therefore most of the energy emitted by the dipole in this region $\left(k_{x}>k_{0}\right)$ is coupled to the HMM high- $k$ modes. In some applications, it is interesting to couple all the energy emitted by the dipole to the far field. The Purcell factor due to the radiative energy of polarization $\alpha$ ( $\alpha$ can be $\perp$ or $\|)\left(Q_{\alpha}\right)$ can be calculated respectively by 


$$
Q_{\alpha}=\int_{0}^{k_{0}} \frac{\mathrm{d} W_{\alpha}}{\mathrm{d} k_{x}} \mathrm{~d} k_{x}
$$

The external quantum efficiency $(\eta)$ of the QE is the ratio of $Q_{\alpha}$ and $P_{\alpha}$,

$$
\eta_{\alpha}=\frac{Q_{\alpha}}{W_{\alpha}}
$$

\subsection{Results}

The most outstanding feature of hyperbolic metamaterials is the existence of photonic states with wavevector $(k)$ much greater than the free space wavevector $\left(k_{0}\right)$. These high$k$ modes can be excited, for instance, by placing a dipole close to the HMM surface [143]. Since the dipole radiation is a superposition of propagating $\left(k_{x} \leq k_{0}\right)$ and evanescent $\left(k_{x} \geq\right.$ $k_{0}$ ) waves, if the dipole is sufficiently close to the surface its evanescent waves will couple to the high-k states of the HMM. Consequently, it becomes crucial to understand the electromagnetic behavior of the HMM for all possible range of $k_{x}$. According to [81], whenever the parameter extraction is carried out in the region of low $k_{x}\left(k_{x} \leq k_{0}\right)$, the spatial dispersion does not really constitute a problem. However, $k_{x} \geq k_{0}$ implies lower effective wavelengths inside the HMM thus making spatial dispersion a subject requiring further investigation. The CPR approach takes this problem into consideration and explores three different cases to highlight its influence on the extracted parameters, namely: the effect of the layers' thickness, the effect of the number of layers and, finally, the effect of the metal/dielectric composition. This will allow us to clearly show how the spatial dispersion behavior for high values of $k_{x}$ is manifested.

\subsubsection{Influence of the Dielectric Thickness}

In this section, we investigate the effect of the dielectric thickness on the extracted parameters obtained with three different approaches, namely: the EMT (solid line, stars), the approach in [81] (dashed lines, hollow symbols), and the CPR approach (solid lines, full symbols). The EMT has been widely used in the literature to calculate the electromagnetic parameters of metal/dielectric stacks [83], and assumes that the dielectric and metal thicknesses are much smaller than the wavelength. In [81], the authors restricted their results to the region $k_{x} \leq k_{0}$. For the sake of comparison, we have extrapolated their approach for cases with $k_{x} \geq \mathrm{k}_{0}$.

In the examples discussed in this section, two different metallic filling factors are considered, i.e., $f f=0.1$ and $f f=0.3$. The wavelength is $780 \mathrm{~nm}$, the dielectric is $\mathrm{TiO} 2\left(\varepsilon_{d}=\right.$ 6.3752), the metal is $\mathrm{Ag}\left(\varepsilon_{m}=-29.36+0.35 \mathrm{j}\right)$, and the number of layers is 21 . Figure 2.6 shows the extracted parameters of the $\mathrm{Ag} / \mathrm{TiO} 2$ stack for $f f=0.1$. The real and imaginary parts of $\varepsilon_{\perp}, \varepsilon_{\|}, \mu_{\perp}$ and $\mu_{\|}$are shown in Figure 2.6 (a-h) for $d=15 \mathrm{~nm}$ (squares), $d=30 \mathrm{~nm}$ (circles) and $d=45 \mathrm{~nm}$ (triangles). For the results obtained with the EMT approximation it is assumed $\mu=1$. Note that since ff is constant, the permittivity obtained with the EMT 

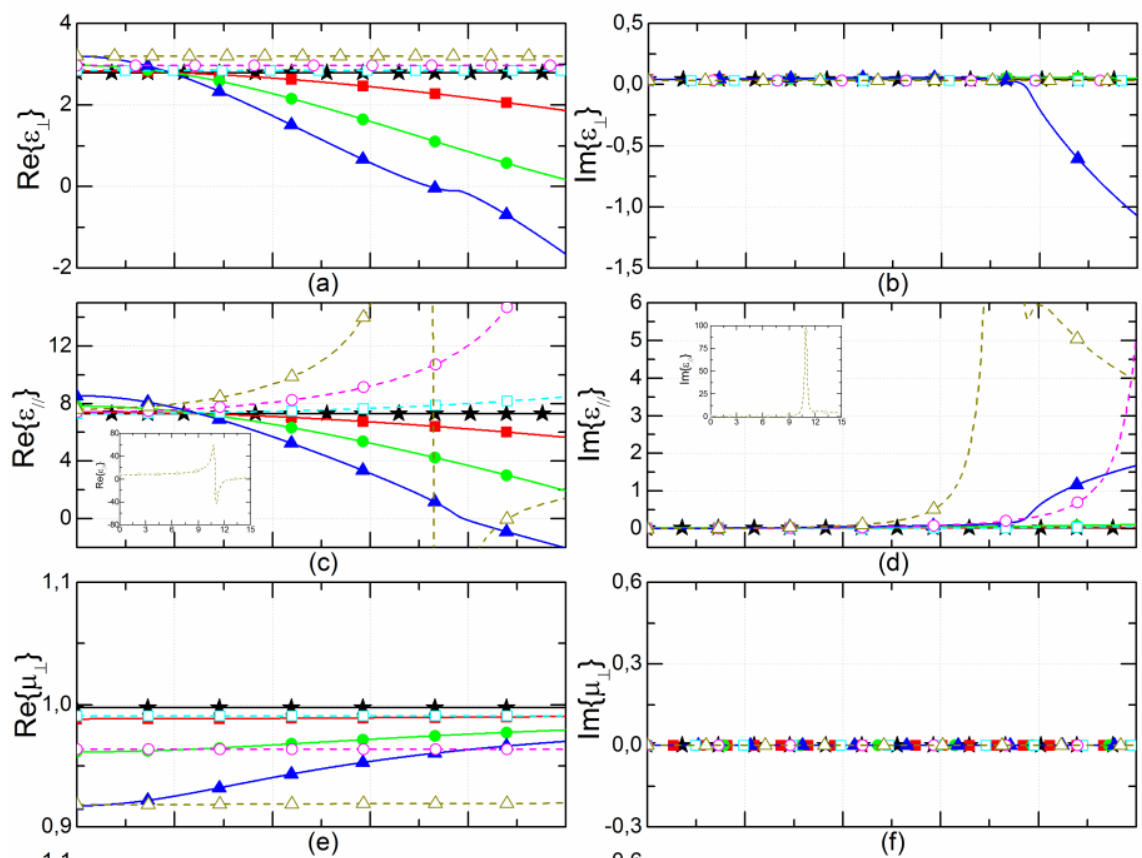

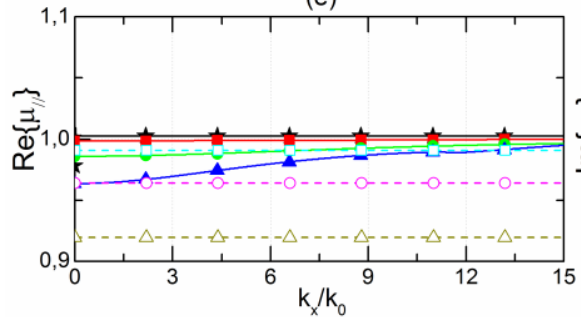

(g)

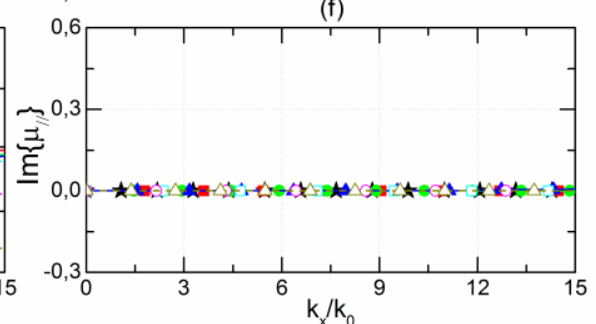

(h)

Figure 2.6. Real (Left column) and imaginary (Right column) parts of the extracted electromagnetic parameters of a layered medium of $\mathrm{Ag} / \mathrm{TiO} 2$ with $\mathrm{f} f=0.1$ for dielectric thickness of 15 (squares), 30 (circles) and $45 \mathrm{~nm}$ (triangles) for [81] (dashed lines, hollow symbols) and the CPR approach (solid lines, full symbols). (a,b) $\varepsilon_{\perp}$ (c,d) $\varepsilon_{\|},(\mathrm{e}, \mathrm{f}) \mu_{\perp}$ and $(\mathrm{g}, \mathrm{h}) \mu_{\|}$. The EMT (solid line, stars) results are also plotted for comparison. The inset in $(\mathrm{c}, \mathrm{d})$ shows the full magnitude of the real and imaginary parts of $\varepsilon_{\|}$, for the $45 \mathrm{~nm}$ case of [81].

is invariant to $k$. In addition, Figure $2.6(\mathrm{e}, \mathrm{g})$ show that the assumption of $\mu=1$ is actually valid only for small values of $d$. The soundness of the CPR approach can be further verified by noting that $\varepsilon_{\perp}$ and $\varepsilon_{\|}$tend to those of the EMT when the dielectric thickness is very small (which is actually the condition for the EMT approximation to be valid). In [81] the authors consider $\varepsilon_{\perp}$ and $\mu_{\perp}$ as constants for any value of $k_{x}$, justified by the absence of spatial dispersion (Figure $2.6(\mathrm{a}, \mathrm{b})$ and $(\mathrm{e}, \mathrm{f})$, dashed lines, hollow symbols). Considering the interval $\left|k_{x} / k_{0}\right|<1$, we notice that $\varepsilon_{\|}$and $\mu_{\|}$present little variation, meaning that spatial dispersion is small in this region (Figure $2.6(\mathrm{c}, \mathrm{d})$ and $(\mathrm{g}, \mathrm{h})$, dashed lines, hollow symbols). However, even in the interval $\left|k_{x} / k_{0}\right|<1$ the parameters extracted with [81] do not agree with those predicted with the CPR approach, especially for thicker 


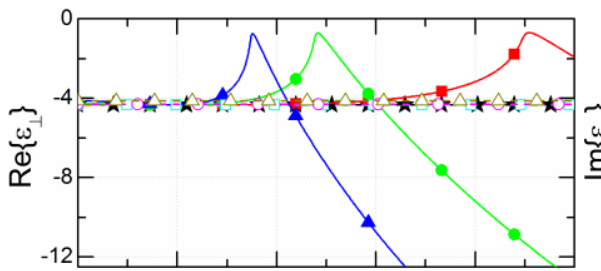

(a)

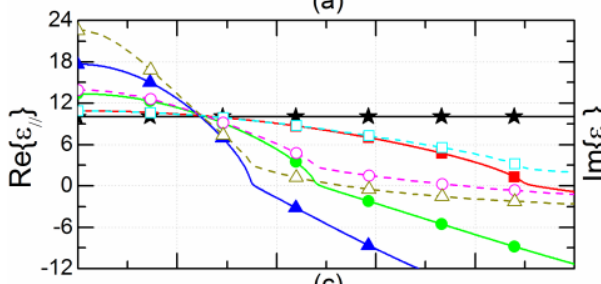

(c)

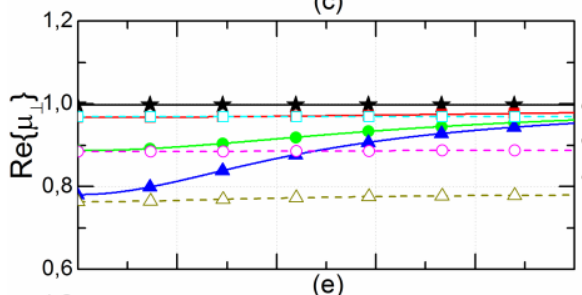

(e)

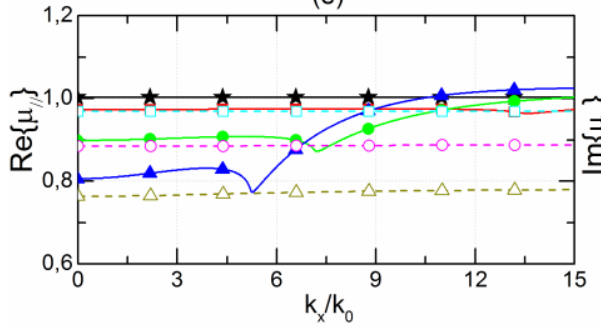

(g)

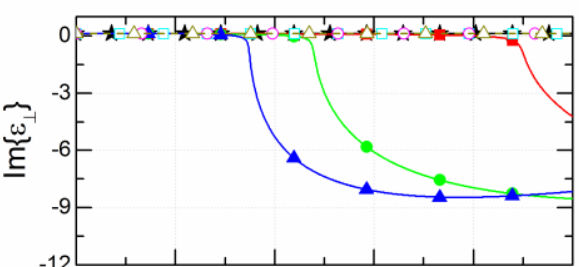

(b)

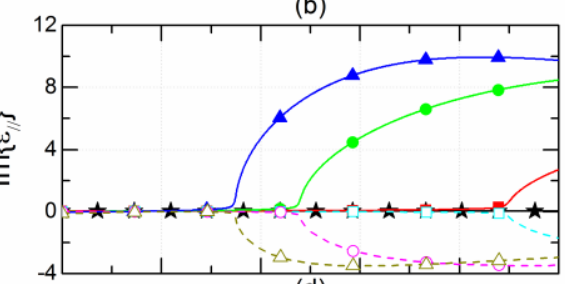

(d)
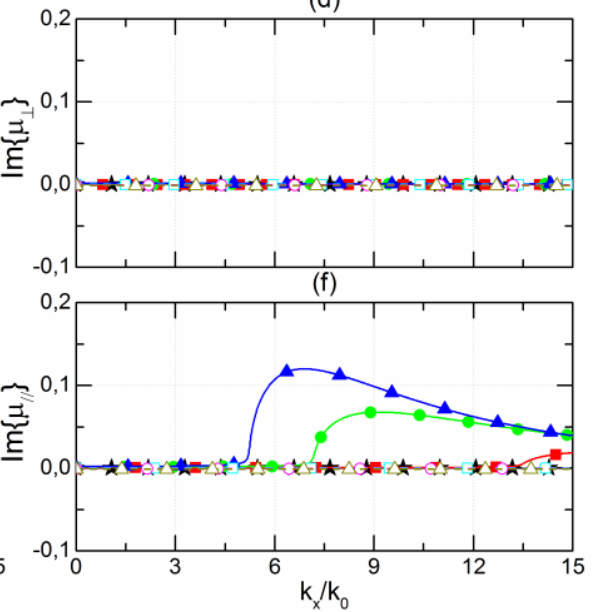

(h)

Figure 2.7. Real (Left column) and imaginary (Right column) parts of the extracted electromagnetic parameters of a layered medium of $\mathrm{Ag} / \mathrm{TiO} 2$ with $\mathrm{ff}=0.3$ for dielectric thickness of 15 (squares), 30 (circles) and $45 \mathrm{~nm}$ (triangles) for [81] (dashed lines, hollow symbols) and the CPR approach (solid lines, full symbols). (a,b) $\varepsilon_{\perp}$, (c,d) $\varepsilon_{\|},(\mathrm{e}, \mathrm{f}), \mu_{\perp}$ and $(\mathrm{g}, \mathrm{h}) \mu_{\|}$. The EMT (solid line, stars) results are also plotted for comparison.

dielectric layers. Yet, if $\varepsilon_{\perp}$ and $\mu_{\perp}$ are assumed constant, $\varepsilon_{\|}$and $\mu_{\|}$will be incorrectly calculated because even small perturbations in $\varepsilon_{\perp}$ and $\mu_{\perp}$ can cause large variations on (2.24) and (2.25). By analyzing the results of the CPR approach, it becomes clear that all electromagnetic parameters vary with $k_{x}$, which characterizes spatial dispersion. In addition, spatial dispersion is so critical that it can even cause $\varepsilon_{\perp}$ and $\varepsilon_{\|}$to change sign $\left(\left|k_{x} / k_{0}\right|=11.9\right.$ for $\varepsilon_{\|}$and $\left|k_{x} / k_{0}\right|=10.8$ for $\varepsilon_{\perp}$, see the plotted triangles curve in Figure 2.6 for $d=45 \mathrm{~nm}$ ). When a sign change occurs, the losses increase very rapidly, and the incident wave interacts almost entirely with the initial layers of the stack. For this reason, the higher the $\left|k_{x} / k_{0}\right|$ ratio, the more the extracted parameters will tend toward those of the first layer (metal in this case). Furthermore, notice that the magnitude of the imaginary part of $\varepsilon_{\perp}$ decreases rapidly for $\left|k_{x} / k_{0}\right|>10.8$, while that of $\varepsilon_{\|}$grows very quickly in the 
region $\left|k_{x} / k_{0}\right|>11.9$ for $d=45 \mathrm{~nm}$ (triangles curve in Figure 2.6). The increase in the metal thickness enhances the magnetic behavior of this material causing a decrease in the permeability values as seen in Figure $2.6(\mathrm{e}, \mathrm{g})$.

Next, we extract the electromagnetic parameters of the TiO2/Ag stack with $f f=0.3$. The real and imaginary parts of $\varepsilon_{\perp}, \varepsilon_{\|}, \mu_{\perp}$ and $\mu_{\|}$are shown in Figure 2.7 (a-h) for $d=15$ $\mathrm{nm}$ (squares), $d=30 \mathrm{~nm}$ (circles) and $d=45 \mathrm{~nm}$ (triangles). Once more, the values of $\varepsilon_{\perp}$ and $\varepsilon_{\|}$tend to those of the EMT (and $\mu_{\perp}$ and $\mu_{\|}$tend to 1) only when the dielectric thickness tends to zero. By observing Figure $2.7(\mathrm{e}, \mathrm{g})$ it becomes clear that the assumption of $\mu=1$ is not valid because the stack does show magnetic response, particularly for thicker layers. Moreover, these results show that the extracted parameters can be incorrectly calculated even for $\left|k_{x} / k_{0}\right|<1$ (Figure $2.7(\mathrm{c}, \mathrm{g})$ ) if spatial dispersion is not taken into account. Despite the differences mentioned above, all three approaches predict a hyperbolic dispersion profile for this structure $\left(\varepsilon_{\perp}<0\right.$ and $\left.\varepsilon_{\|}>0\right)$ for low values of $\left|k_{x} / k_{0}\right|$. Nevertheless, for $d=30 \mathrm{~nm}$, the CPR approach predicts a change in the shape of the dispersion curve for $\left|k_{x} / k_{0}\right|>7.5$ because $\varepsilon_{\|}$becomes smaller than zero (Figure 2.7 (c)). The same phenomenon occurs for $d=45 \mathrm{~nm}$ and $\left|k_{x} / k_{0}\right|>5.3$, and for $d=15 \mathrm{~nm}$ and $\left|k_{x} / k_{0}\right|$ $>14$. As explained, the electromagnetic parameters tend to those of the first layer (a metal in this case, with $\varepsilon_{\perp}<0$ and $\left.\varepsilon_{\|}<0\right)$ for increasing $\left|k_{x} / k_{0}\right|$ values. The peaks and inflection points in (Figure 2.7 (a) and (c), respectively, as well as the valleys in (Figure 2.7 (g) only appear if spatial dispersion is properly taken into account in the parameter retrieval procedure.

\subsubsection{Influence of the number of Layers}

In this section, the effect of the number of layers on the design and performance of hyperbolic metamaterials is investigated. Again, the results are obtained with the EMT (solid line, stars), [81] (dashed lines, hollow symbols), and the CPR approach (solid lines, full symbols). The structure consists of a stack of $\mathrm{Ag} / \mathrm{TiO} 2$ with $d=20 \mathrm{~nm}, f f=0.3$, and wavelength $\lambda=780 \mathrm{~nm}$. Different number of layers are considered, i.e., 3 (squares), 15 (circles) and 27 (triangles). The results for the real and imaginary parts of $\left(\varepsilon_{\|}, \varepsilon_{\perp}<0\right)$ are shown in Figure $2.8(\mathrm{a}, \mathrm{c})$ and $(\mathrm{b}, \mathrm{d})$, respectively. Regardless of the approach, the change in the number of layers does not modify the effective parameters, thus keeping $R_{\Delta}$ and $Z_{\Delta}$ unaltered according to (2.15) and (2.16). However, the scattering parameters do change because the total thickness of the slab increases with the increase in the number of layers according to (2.18) and (2.19). Additionally, Figure 2.8 (a) and (b) show how the spatial dispersion impacts the magnitude of the retrieved parameters, an effect that is not observed with the EMT approximation or with the approach of [81] for $\varepsilon_{\|}$. When $d$ is small, [81] shows good accuracy only in the region $\left|k_{x} / k_{0}\right|<1$.

\subsubsection{Analysis of the Dispersion Curves}

As discussed earlier, hyperbolic metamaterials can support solutions with $k_{x} \gg k_{0}$. However, because of spatial dispersion, there are regions of high $k_{x}$ where the layered 

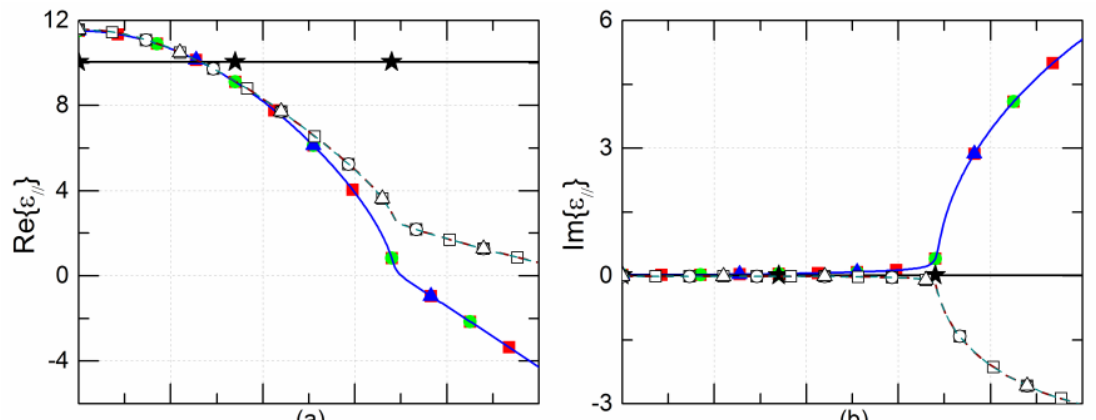

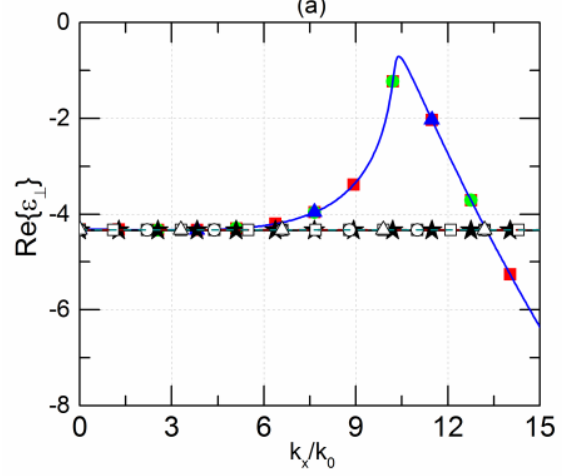

(c)

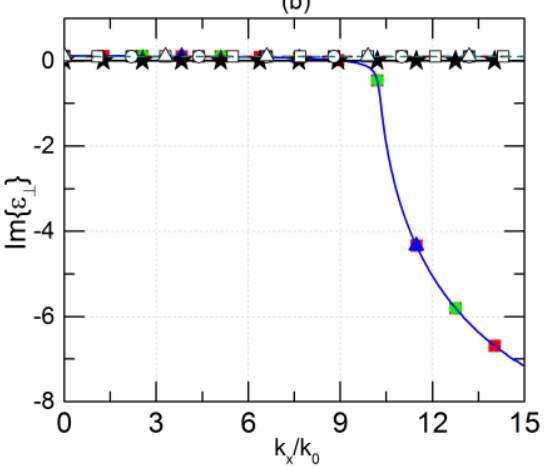

(d)

Figure 2.8. Real (left column) and imaginary (right column) parts of (a) $\varepsilon_{\|}$and (b) $\varepsilon_{\perp}<0$ for 3 (squares), 15 (circles) and 27 (triangles) layers for [81] (dashed lines, hollow symbols) and the CPR approach (solid lines, full symbols). The EMT (solid line, stars) results are also plotted for comparison

medium is highly lossy and wave propagation is not supported. In order to better understand this condition, we investigate a stack of $\mathrm{Ag} / \mathrm{SiO} 2\left(\varepsilon_{d}=2.126\right.$ and $\varepsilon_{m}=-16.075+$ $0.437 \mathrm{j}$ ) with $f f=0.3$ and $f f=0.5$ at $\lambda=600 \mathrm{~nm}$ for different dielectric thicknesses $(8,16,24$, and $32 \mathrm{~nm}$ ) and TM polarization. The real (left column) and imaginary (right column) parts of the dispersion curves obtained with the CPR approach (solid lines, full symbols) are shown in Figure 2.9. Results with the EMT (solid line, stars) and [81] (dashed lines, hollow symbols) are also shown for comparison. Note that the dispersion curve obtained with [81] is hyperbolic and tends to that of the EMT as the dielectric thickness decreases. It is important to highlight that since both [81] and EMT do not account for spatial dispersion, the imaginary part of their respective propagation constants (Figure $2.9(\mathrm{~b}, \mathrm{~d})$ ) are close to zero in the region of $\left|k_{x} / k_{0}\right|>2.5$. Likewise, spatial dispersion has negligible effect on the CPR approach only when $\left|k_{x} / k_{0}\right|<2.5$. In sharp contrast to EMT and [81], $\mathrm{CPR}$ exhibits a flattening of the dispersion curve (and the consequent increase in its imaginary part) beyond a threshold value of $\left|k_{x} / k_{0}\right|$ that varies with thickness leading to the absence of propagating modes inside the HMM. For example, as seen in Figure 2.9 for a thickness of $16 \mathrm{~nm}$, flattening occurs for $\left|k_{x} / k_{0}\right|>5$. Also notice that as $\left|k_{x} / k_{0}\right|$ increases the electromagnetic parameters tend to those of the metal (i.e., $S_{11}$ tends to -1 while $S_{21}$ tends to 0$)$. Therefore, (2.21) can be rewritten as 


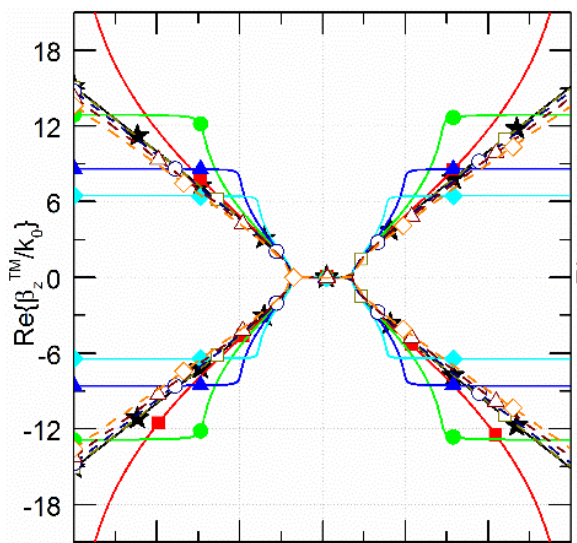

(a)

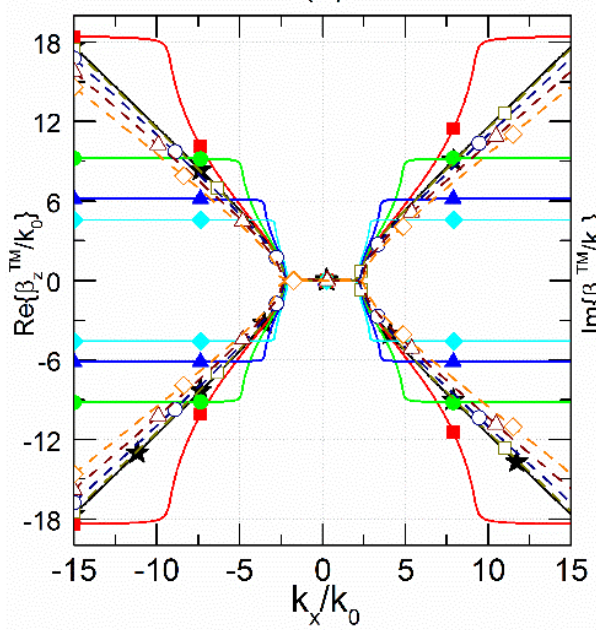

(c)

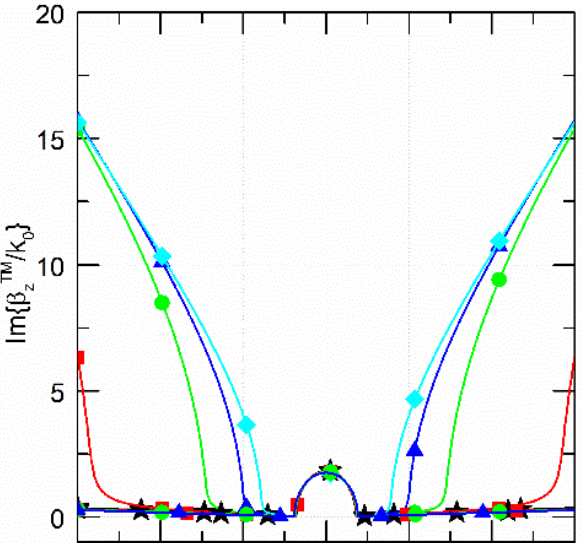

(b)

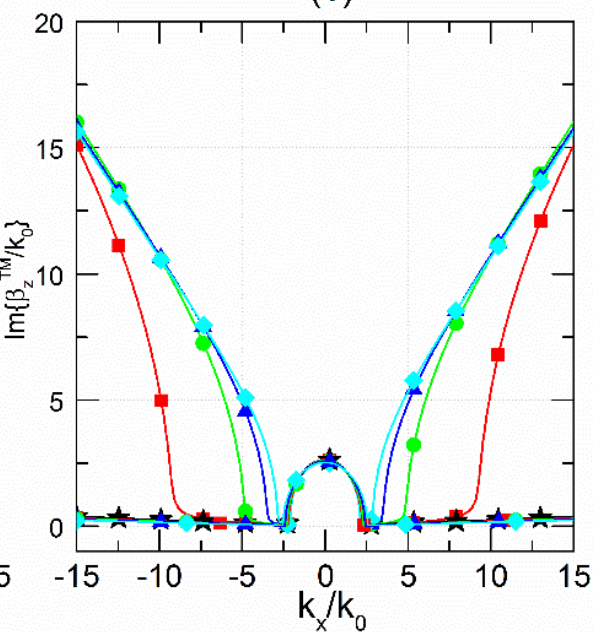

(d)

Figure 2.9. Real (left column) and imaginary (right column) parts of the dispersion curves of a $\mathrm{Ag} / \mathrm{SiO} 2$ stack with $f f=0.3(\mathrm{a}, \mathrm{b})$ and $f f=0.5(\mathrm{c}, \mathrm{d})$. The dielectric thicknesses (used as a parameter) is $8 \mathrm{~nm}$ (squares), $16 \mathrm{~nm}$ (circles), $24 \mathrm{~nm}$ (triangles) and $32 \mathrm{~nm}$. (diamonds) for [81] (dashed lines, hollow symbols) and the CPR approach (solid lines, full symbols). Results from the EMT (solid line, stars) are also shown for comparison.

$$
\beta_{z}^{\chi} \stackrel{k_{x} \rightarrow \infty}{\longrightarrow} \frac{1}{t}\left\{\mathrm{jlog}\left(\left|\frac{1}{S_{21}^{\chi}}\left[1-\left(S_{21}^{\chi}\right)^{2}+\left(S_{11}^{\chi}\right)^{2}\right]\right|\right)+\pi(2 n-1)\right\}
$$

Taking only the real part of (2.34) we get

$$
\operatorname{Re}\left\{\beta_{z}^{\chi}\right\} \stackrel{k_{x} \rightarrow \infty}{\longrightarrow} \frac{\pi(2 n-1)}{t}=m \frac{\pi}{t} .
$$

The integer $m$ can be calculated as function of the number of layers $(N)$, 
CHAPTER 2 -

HOMOGENIZATION OF UNIAXIAL ANISOTROPIC METAMATERIALS

$$
m=\text { floor }\left(\frac{N}{2}\right)
$$

Since $t=d /(1-f f)$ floor $(N / 2)$, the values where the curves flattens out can be approximated as

$$
\operatorname{Re}\left\{\beta_{z}^{\chi}\right\} \stackrel{k_{x} \rightarrow \infty}{\longrightarrow} \frac{\pi(1-f f)}{d}
$$
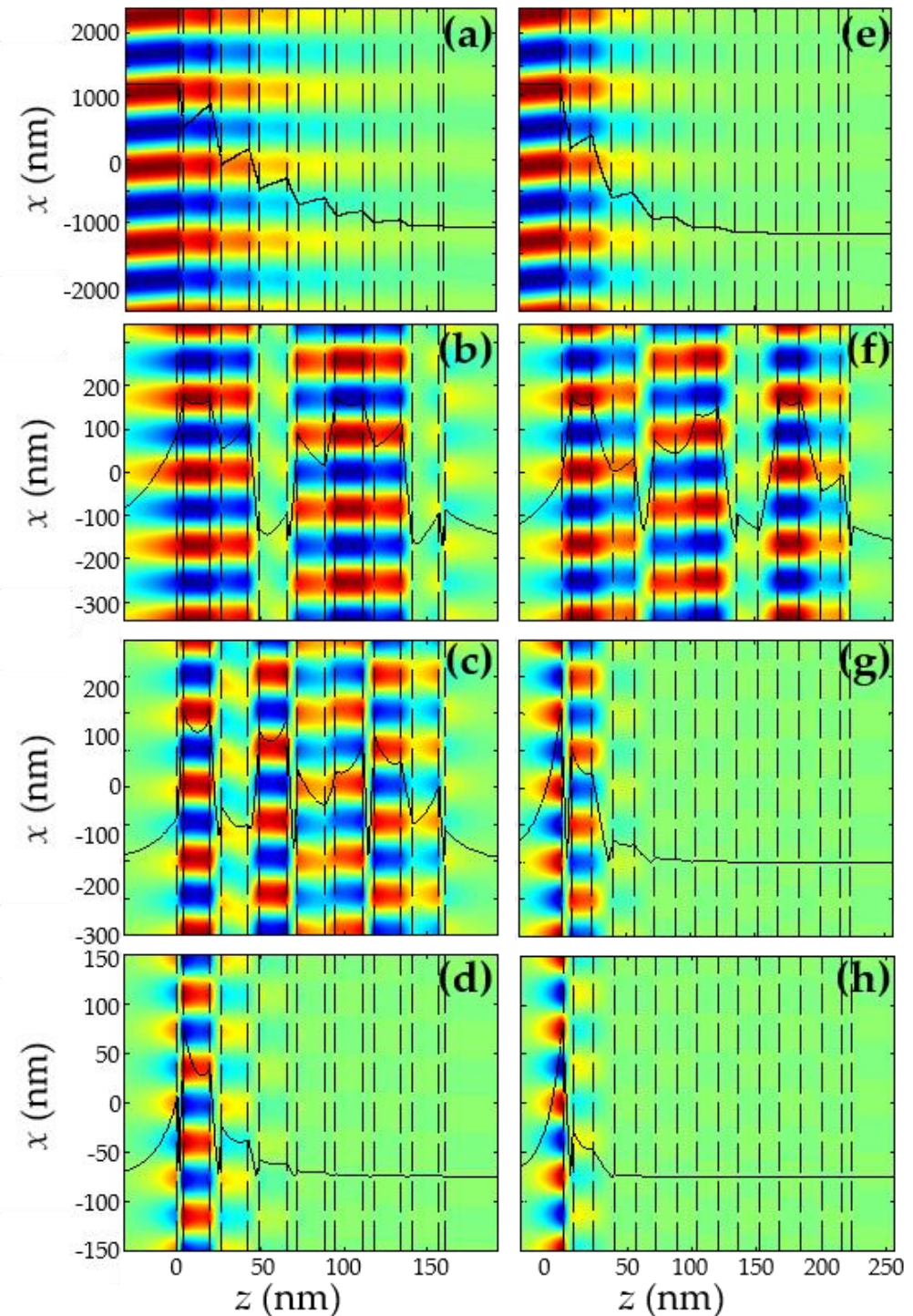

Figure 2.10. $H_{y}$ profile for $f f=0.3((\mathrm{a}, \mathrm{d}))$ and for $f f=0.5((\mathrm{e}, \mathrm{h}))$, for $d=16 \mathrm{~nm}$ and different $\left|k_{x} / k_{0}\right|$ values $\left(\left|k_{x} / k_{0}\right|=0.5\right.$ in $(\mathrm{a}, \mathrm{e}),\left|k_{x} / k_{0}\right|=3.5 \mathrm{in}(\mathrm{b}, \mathrm{f}),\left|k_{x} / k_{0}\right|=6$ in $(\mathrm{c}, \mathrm{g})$ and $\left|k_{x} / k_{0}\right|=8$ in (d, (h)). The solid black line represents the amplitude of $H_{y}$, and the dashed lines represent the boundaries of the layers. The HMM is assumed immersed in air. 
According to (2.37), the flat regions start sooner (at lower $\left|k_{x} / k_{0}\right|$ values) either for thicker layers or for higher filling factors. It is important to notice that the number of layers does not influence the point where the flattening behavior starts. The imaginary part (Figure 2.9 (b)) is also more significant in this region, indicating a stronger field interaction with the metal layers. The flattening out of the dispersion curve becomes more evident for a fill factor $f f=0.5$, as shown in Figure $2.9(\mathrm{c}, \mathrm{d})$ and according to (2.37). This analysis shows that the $\mathrm{Ag} / \mathrm{SiO}_{2}$ stack is not a good candidate for hyperbolic metamaterial for two reasons: 1) spatial dispersion can be mitigated with low fill factors but only at the expense of impractically thin metal layers; 2 ) high fill factors make spatial dispersion particularly problematic.

An alternative way to visualize the electromagnetic behavior of the $\mathrm{Ag} / \mathrm{SiO}_{2}$ stack is by observing the magnetic field profile inside the medium calculated using the TMT. Figure 2.10 shows the real part of $H_{y}$ for $f f=0.3$ (a-d) and for $f f=0.5(\mathrm{e}-\mathrm{h})$, for $d=16 \mathrm{~nm}$ and different values of $\left|k_{x} / k_{0}\right|$ (i.e., $\left|k_{x} / k_{0}\right|=0.5$ in $(\mathrm{a}, \mathrm{e}),\left|k_{x} / k_{0}\right|=3.5$ in $(\mathrm{b}, \mathrm{f}),\left|k_{x} / k_{0}\right|=6$ in $(\mathrm{c}, \mathrm{g})$ and $\left|k_{x} / k_{0}\right|=8$ in $\left.(\mathrm{d}, \mathrm{h})\right)$. The solid black line represents the amplitude of $H_{y}$ while the dashed black line represents the boundaries of the layers. According to Figure 2.10, when $\left|k_{x} / k_{0}\right|=0.5$ for both $f f=0.3$ and $f f=0.5$, the wave inside the stack is lossy (especially for $f f$ $=0.5$, since the imaginary part of $\beta_{z}^{T M}$ is higher). This behavior is also observed in Figure $2.10(a, b)$, which shows the energy being dissipated as the wave propagates though the HMM. When $\left|k_{x} / k_{0}\right|=3.5$, the wave experiences low propagation loss for both values of $f f$ (see Figure 2.10). Moreover, Figure $2.10(\mathrm{~b}, \mathrm{f})$ show that although evanescently decaying in air $\left(\left|k_{x} / k_{0}\right|>1\right)$, the wave couples to and propagates through the HMM. By further increasing $\left|k_{x} / k_{0}\right|$ to 6 , it can be observed that wave propagation only happens for $f f=0.3$ (as shown Figure $2.10(\mathrm{c}, \mathrm{g})$ ). This occurs because for $f f=0.5$ the metamaterial is already in the flat region of the dispersion curve (see Figure $2.10(\mathrm{c}, \mathrm{d})$ ) causing the magnitude of $H_{y}$ to strongly decay. Increasing $\left|k_{x} / k_{0}\right|$ even further $\left(\left|k_{x} / k_{0}\right|=8\right)$, puts the metamaterial in the flat region of the dispersion curve for both $f f=0.3$ and 0.5 . As a result, the wave amplitude decays exponentially as shown in Figure $2.10(\mathrm{~d}, \mathrm{~h})$. In conclusion, Figure 2.10 provides a good indication, both qualitatively and quantitatively, that the CPR approach is indeed able to predict accurately the behavior of hyperbolic metamaterials.

Next, we investigate two stack configurations often used for hyperbolic media [55], [143]: $\mathrm{Ag} / \mathrm{TiO}_{2}$ and $\mathrm{Au} / \mathrm{Si}$. The polarization is $\mathrm{TM}$ and the wavelength is $\lambda=600 \mathrm{~nm}$, which corresponds to $\varepsilon_{m}=-16.075+0.437 \mathrm{j}$ and $\varepsilon_{d}=6.786$ for $\mathrm{Ag} / \mathrm{TiO} 2$, and $\varepsilon_{m}=-9.781+2.045 \mathrm{j}$ and $\varepsilon_{d}=15.51+0.018 \mathrm{j}$ for $\mathrm{Au} / \mathrm{Si}$. Figure $2.11(\mathrm{a}-\mathrm{h})$ show the dispersion curves for both stacks with $f f=0.2,0.3,0.4$ and 0.5 . The dielectric thickness is used as a parameter with values of $8,16,24$, and $32 \mathrm{~nm}$. Figure $2.11(\mathrm{a}, \mathrm{b})$ show that the $\mathrm{Ag} / \mathrm{TiO} 2$ stack behaves essentially as a dielectric (not hyperbolic), and that for $\left|k_{x} / k_{0}\right|<3$ the dielectric thickness does not play an important role in the definition of the dispersion curve. The $\mathrm{Ag} / \mathrm{TiO}_{2}$ stack behavior, however, does become hyperbolic for $f f=0.4$ and 0.5 as seen in Figure $2.11(\mathrm{c}, \mathrm{d})$, respectively, in spite of the flattening of the dispersion curve. Regarding the Au/Si stack, the EMT approximation predicts the behavior of this structure as essentially dielectric for $f f=0.2$ and $f f=0.3$. A similar behavior is also observed with the CPR approach, as seen in Figure $2.11(\mathrm{e}, \mathrm{f})$, which predicts an elliptical dispersion profile for both these fill factors. By increasing ff even further to 0.4 and 0.5 as shown in Figure 2.11 (g,h), respectively, the 

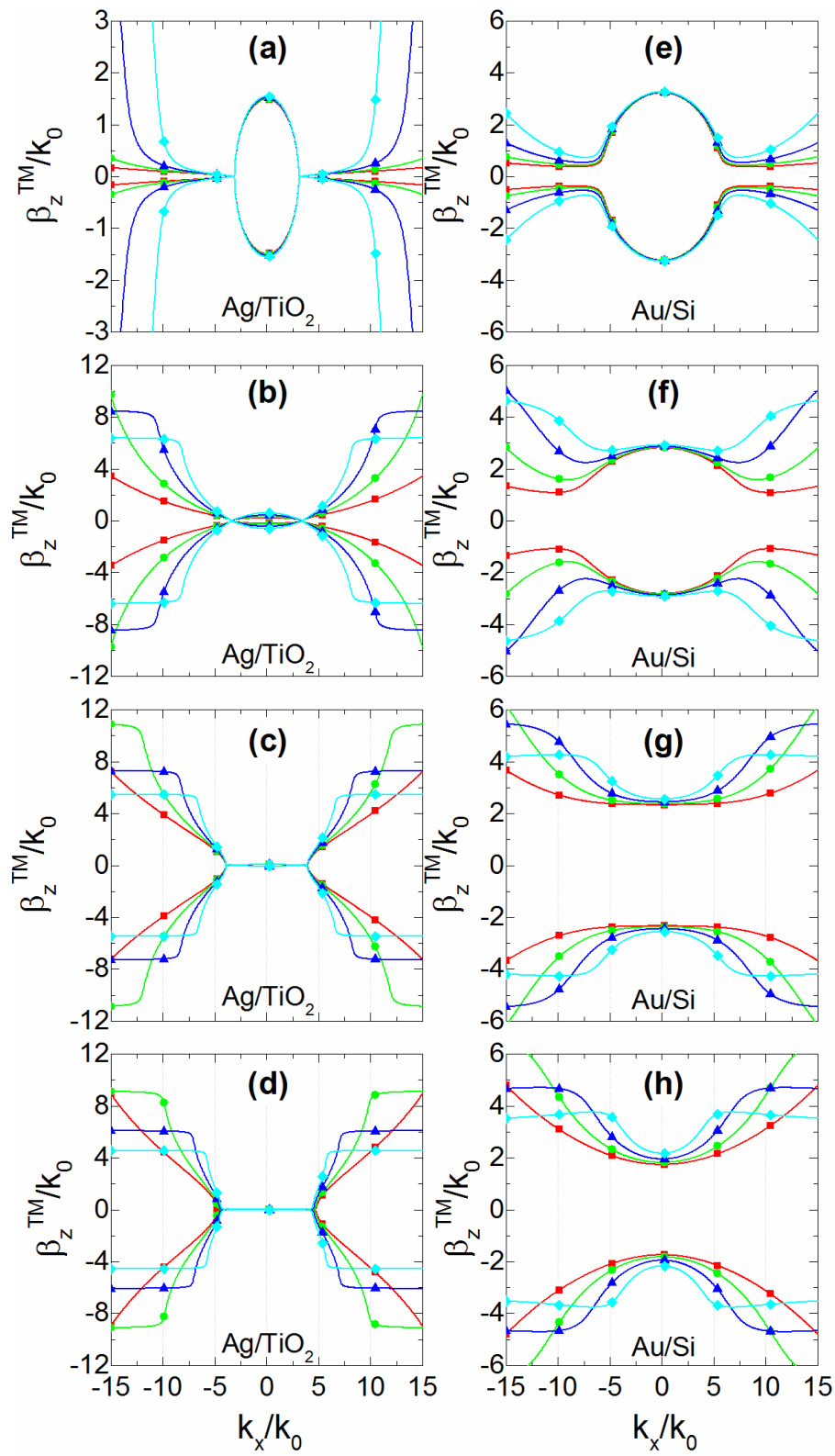

Figure 2.11. Dispersion curves for $\mathrm{Ag} / \mathrm{TiO} 2((\mathrm{a})-(\mathrm{d}))$ and $\mathrm{Au} / \mathrm{Si}((\mathrm{e})-(\mathrm{h}))$ stacks with $(\mathrm{a}, \mathrm{e}) f f=0.2$, $(\mathrm{b}, \mathrm{f}), f f=0.3,(\mathrm{c}, \mathrm{g}), f f=0.4$ and $(\mathrm{d}, \mathrm{h}), f f=0.5$. The dielectric thicknesses (used as a parameter) are $8 \mathrm{~nm}$ (squares), $16 \mathrm{~nm}$ (circles), $24 \mathrm{~nm}$ (triangles) and $32 \mathrm{~nm}$ (diamonds). Note that at $f f=0.4$ and $f f=0.5, \mathrm{Ag} / \mathrm{TiO} 2$ corresponds to a single-sheet hyperboloid while $\mathrm{Au} / \mathrm{Si}$ corresponds to a two-sheet hyperboloid surface.

dispersion curves become hyperbolic. Moreover, as $d$ (or ff) increases so does the metal thickness. Thus, the metamaterial loss becomes significant and the dispersion curve once again flattens out. 
In summary, these results show that spatial dispersion not only is always present, but also that it plays an important role on the dispersion profile for any $\left|k_{x} / k_{0}\right|$ ratio. More importantly, they show that this effect cannot be neglected particularly in regions where the dispersion region is flat. Finally, since this flat region strongly depends on the metal thickness (i.e., it occurs for lower $\left|k_{x} / k_{0}\right|$ values when thicker metal layers are used), a careful design of the metamaterial is required to avoid this issue.

\subsection{Calculation of the Purcell Factor}

One particularly important application of hyperbolic metamaterials is on the enhancement of the Purcell factor [38], [40], [55]. This is true because hyperbolic metamaterials support high $k_{x}$ values and, consequently, a much higher number of electromagnetic states [37] than ordinary materials do. As a result, the decay rate for an atom placed above these materials can be increased, thus enhancing the Purcell factor. Considering an atom at a distance $q$ from a surface, the Purcell factor for the perpendicular orientation can be calculated using (2.29).

The reflection coefficient can be calculated in four different ways: i) using (2.1)-(2.5), which is numerically precise and accurate and treated here as benchmark [38], [40], [55]; ii) using (2.18) and (2.19) with the help of the EMT approximation; iii) using [81], and iv) with the CPR approach. In order to show the differences among these four approaches, we consider $\mathrm{Rb}$ atoms $10 \mathrm{~nm}$ above the HMM surface, for emission wavelengths of 780 $\mathrm{nm}$ and $422 \mathrm{~nm}$. The $\mathrm{HMM}$ chosen is the $\mathrm{Ag} / \mathrm{TiO}_{2}$ stack with two distinct filling factors, i.e., $f f=0.3$ and $f f=0.5$, and $N=13$ layers. Figure 2.12 (a) shows the Purcell factor calculated for $780 \mathrm{~nm}$. In both cases $(f f=0.3$ and $f f=0.5)$ the benchmark results (squares) and the CPR approach (circles) are in excellent agreement, indicating that the parameter extraction procedure proposed here is very accurate. The results from (ii) (stars) and (iii) (triangles), on the other hand, deviate considerably from the benchmark solution in almost the entire dielectric thickness range (except for very thin layers) as a result of spatial dispersion not being taken into account (see Figure 2.9 and Figure 2.11). Consequently, the Purcell factor calculated with these approaches is overestimated. The approaches (i) and (iv) are both capable of predicting this flattening behavior and correctly showing the decrease of the Purcell factor as the thickness $d$ increases. The maximum Purcell factor for $\lambda=780 \mathrm{~nm}$ occurs for $f f=0.3$. As mentioned previously in this chapter, the higher the value of $f f$, the sooner the at region in the dispersion curve will start and, consequently, the higher the propagation loss. This explains why the Purcell factor is larger for $f f=0.3$ than it is for $f f=$ 0.5. Finally, Figure 2.12 (b) shows the Purcell factor calculated for the emission wavelength of $422 \mathrm{~nm}$. Observe that both approaches (i) and (iv) show an excellent agreement throughout the entire thickness interval. The results from (ii) and (iii), on the other hand, deviate considerably in almost the entire dielectric thickness range (except for very thin layers). Again, this is due to spatial dispersion. Differently from the previous example (Figure 2.12 (a)), for $\lambda=422 \mathrm{~nm}$ the Purcell factor is higher for $f f=0.5$ than for $f f$ $=0.3$. This is a direct consequence of the stack dispersion profile not being hyperbolic for $f f=0.3$. 


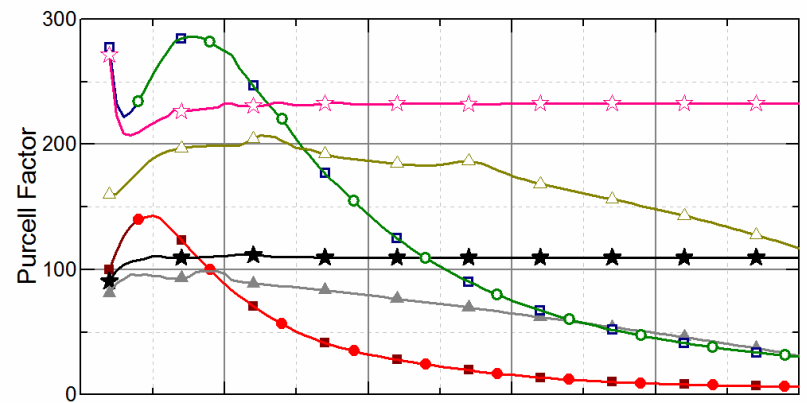

(a)

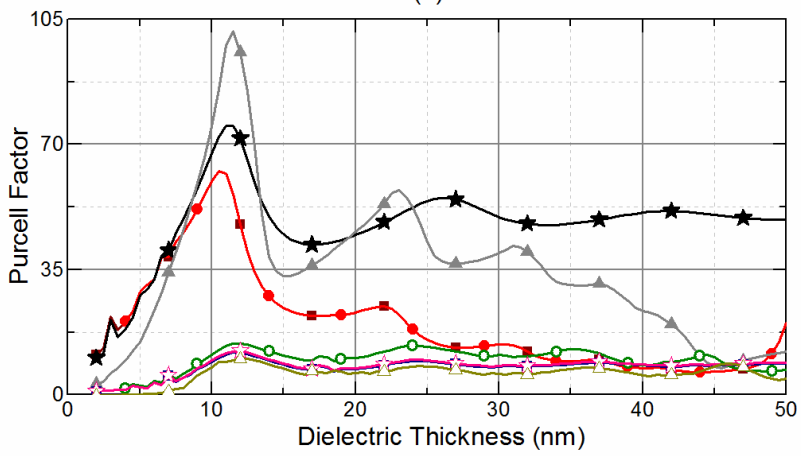

(b)

Figure 2.12. Purcell factor calculated using (i) the benchmark approach (squares), ii the EMT approach (stars), (iii) [81] (triangles), and (iv) the CPR approach (circles). The calculation is carried out for a $\mathrm{Rb}$ atom $10 \mathrm{~nm}$ above the surface of a $\mathrm{Ag} / \mathrm{TiO} 2$ stack with $f f=0.3$ (hollow symbols) and 0.5 (full symbols) for $\lambda=780 \mathrm{~nm}$ (a) and $422 \mathrm{~nm}$ (b).

\subsection{Chapter conclusion}

In this chapter we have proposed a parameter retrieval approach in which all electromagnetic parameters of the medium are obtained and where spatial dispersion is properly taken into account. We have also relaxed the constraint on nonmagnetic metal/dielectric metamaterials. This approach was successfully applied to different metal/dielectric stacks in order to address the effects of the layer thickness, number of layers, and metal/dielectric choice on the spatial dispersion. The results have demonstrated that all investigated metal/dielectric stacks have a clear magnetic response, particularly for thicker layers. Moreover, for large $\left|k_{x} / k_{0}\right|$ the dispersion curve shows a flat region with a large imaginary part that arises due to the absence of propagating modes inside the metamaterial. This flat region is also strongly dependent on the thickness of the layers. The thicker the metal/dielectric layer is, the sooner (for lower $\mid k_{x}$ $\left|k_{0}\right|$ values) the flat and lossy region appears. This behavior is a direct manifestation of the spatial dispersion and strongly affects the accuracy with which the Purcell factor is calculated. In order to illustrate this issue, we have calculated the Purcell factor for $\mathrm{Rb}$ atoms $10 \mathrm{~nm}$ above the surface of a $\mathrm{Ag} / \mathrm{TiO}_{2}$ stack with two distinct filling factors, i.e., $\mathrm{ff}=$ 
0.3 and $f f=0.5$, and $N=13$ layers, for two emission wavelengths, i.e., $422 \mathrm{~nm}$ and $780 \mathrm{~nm}$. The results were compared with three different approaches, namely, the benchmark result, the approach in [81], and the EMT approach. We have shown that if spatial dispersion is not properly taken into account, the Purcell factor is overestimated, as observed with both [81] and EMT approaches. However, the CPR approach has shown excellent agreement with the benchmark results. 



\section{Chapter 3 \\ Enhancing the \\ Purcell factor using HMM}




\section{Chapter 3 - Summary}

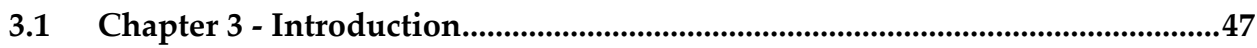

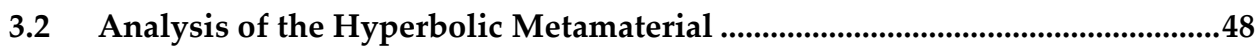

3.3 Single Cy7 molecule embedded in a PMMA layer..............................................52

3.4 Homogenous distribution of $\mathrm{Cy} 7$ inside the PMMA layer ................................54

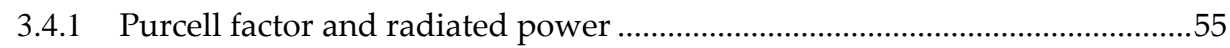

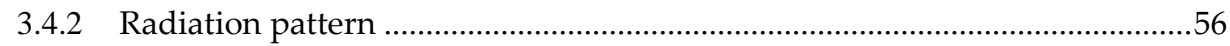

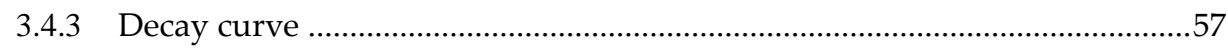

3.5 Influence of the PMMA thickness in system's Purcell factor and quantum efficiency ...........................................................................................................................58

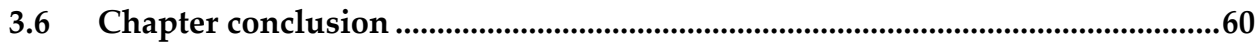




\subsection{Chapter 3 - Introduction}

In this Chapter, we demonstrate how the Purcell factor of a QE can be increased utilizing HMM. For the proposed study, we target the fluorescent molecule Cy7, whose emission wavelength is close to that of the $\mathrm{Rb}$ atom investigated in subsection 2.4. Nonetheless, differently from $\mathrm{Rb}$, Cy7 needs to be embedded in a host polymer layer which is assumed here to be deposited on the top of the HMM. The polymer finite thickness gives rise to reflection and transmission patterns that need to be properly accounted for in the calculation of $P, W_{\text {rad }}$ and $\eta$. The set of equations that govern the electromagnetic behavior of a QE as function of its position inside the polymer layer is fully addressed in this chapter.

Since each QE contributes to the system's decay behavior, knowing $P$ and $\eta$ as function of position is important because in real applications the QEs are randomly distributed and oriented inside the host layer. Based on the knowledge of $P$ and $\eta$ in each position, we demonstrate how to compute the decay curve $g(t)$ of a system composed of multiple QEs uniformly distributed inside the host layer and show that $g(t)$ is more influenced by the QEs with high $\eta$, since they contribute the most with the power measured in the far field.

We also show that both $P$ and $\eta$ are highly dependent on the distance from the HMM surface. While $P$ increases for QEs closer to the HMM surface, $\eta$ decreases as most of the dissipated power is coupled to the HMM's high- $k$ modes. Because of this variation, the polymer thickness has an enormous impact on the system's $\eta$ and $P$, and consequently to $g(t)$. Therefore, controlling the radiation parameters of QEs via polymer layer thickness is an important issue also addressed in this chapter.

The remainder of this chapter is organized as follows. Section 3.1 presents the main design concepts of HMMs and the mathematical formalism to calculate $P$ and $\eta$ of a QE embedded in a bounded media. In section 3.2, the proposed model is applied to the analysis of the QE electromagnetic behavior inside the host layer. In section 3.3 a uniform distribution of QEs is assumed inside the host layer so that the mathematical formalism for the computation of $g(t)$ can be obtained. Section 3.4 describes the influence of the host film thickness on $g(t)$. Finally, section 3.5 presents some concluding remarks. 


\subsection{Analysis of the Hyperbolic Metamaterial}

The focus here is to propose a HMM capable of increasing the Purcell factor of the fluorescent molecule Cy7, which is a molecule largely used for medical applications [117]-[119]. The Cy7 absorption (squares) and emission (circles) peaks (obtained from Cy7 datasheet) occur at $750 \mathrm{~nm}$ and $773 \mathrm{~nm}$, respectively, as shown in Figure 3.1. Therefore, to be effective, the HMM design requires a large PDoS at Cy7 emission wavelength. In chapter 2 it was shown that a HMM consisting of 13 alternating layers of $\mathrm{Ag} / \mathrm{TiO}_{2}$ with $f f=0.3$ can increase the Purcell factor $P$ of a $\mathrm{Rb}$ atom located $10 \mathrm{~nm}$ above the HMM's surface by a factor of 300 . Fortunately, both Cy7 (773nm) and Rb (780nm) emission wavelengths are close to each other. Since HMMs are well-known for their broadband behavior, we carry out the Cy7 HMM design based on the design procedure described in subsection 2.4 .

To ease the fabrication process, the number of layers is reduced from 13 to 7 alternating layers. The materials adopted in this design (with permittivities obtained at 773nm) are $\mathrm{Ag}(\varepsilon=-25+1.71 \mathrm{j})$ and $\mathrm{TiO}_{2}\left(\varepsilon_{m}=6.38\right)$ for the alternating layers and $\mathrm{SiO}_{2}\left(\varepsilon_{d}=2.25\right)$ for the substrate, as shown in Figure 3.2. Furthermore, to obtain ff around 0.3, the Ag and $\mathrm{TiO}_{2}$ layer thicknesses are $10 \mathrm{~nm}$ and $25 \mathrm{~nm}$, except for the Ag top layer, whose thicknesses is $5 \mathrm{~nm}$. The Cy7 molecules are mixed with the polymer host and spin-coated on the top of the HMM. The QEs need to be close to the HMM surface to enhance $P$, which requires the polymer thickness to be as thin as possible. In this sense, we choose the Microchem 950 polymethylmethacrylate A Resists A2 (PMMA, $\varepsilon_{h}=2.25$ ) polymer, which can be spun-off to a thickness as thin as $60 \mathrm{~nm}$ at $5000 \mathrm{rpm}$ for 40s. Therefore, we set the PMMA thickness (h) to $60 \mathrm{~nm}$, as shown in Figure 3.2.

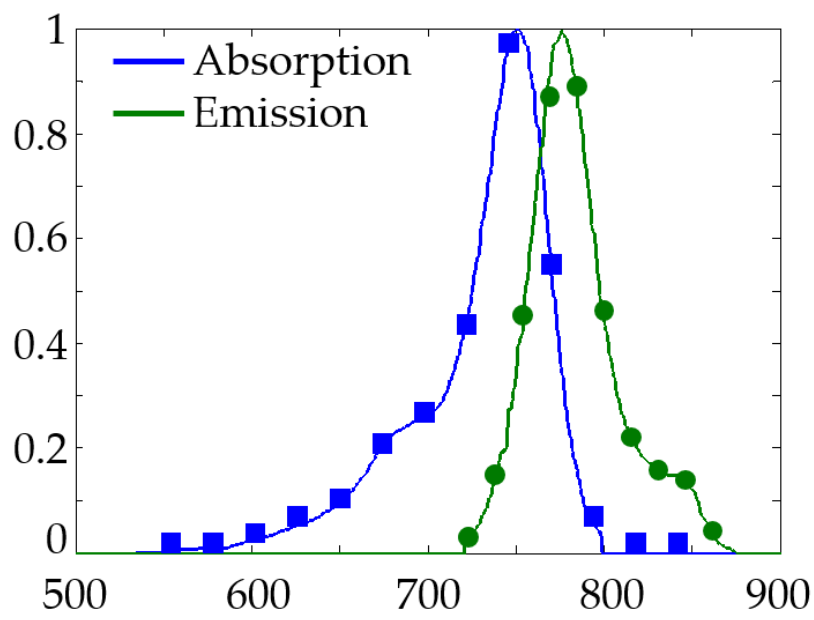

Figure 3.1. Absorption (red) and emission (blue) of the Cy7 


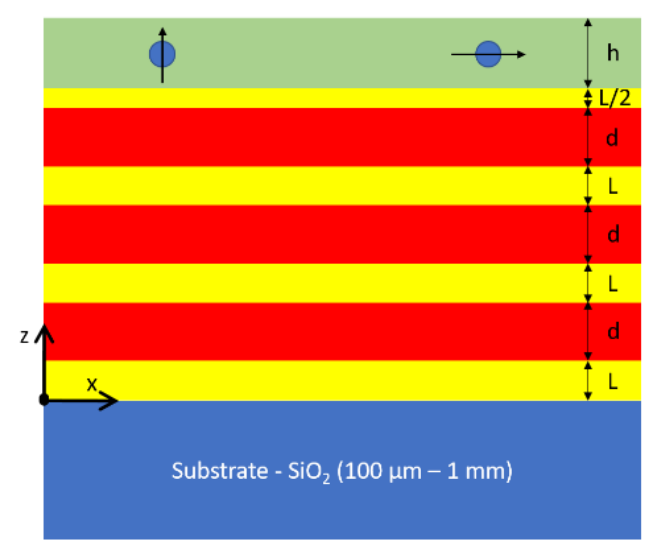

$$
\begin{aligned}
& \mathrm{d}=25 \mathrm{~nm} \pm 2,0 \mathrm{~nm} \\
& \mathrm{~L}=10 \mathrm{~nm} \pm 1,0 \mathrm{~nm} \\
& \mathrm{~h}=60 \mathrm{~nm}
\end{aligned}
$$

Wafer Size: $1.5 \mathrm{~cm}$ x $1.5 \mathrm{~cm}$

\section{Silver (Ag)}

Titanium Dioxide $\left(\mathrm{TiO}_{2}\right)$

PMMA $(60 \mathrm{~nm})$

Figure 3.2. Proposed HMM consisting of 7 alternating layers of silver $\left(\mathrm{Ag}, \varepsilon_{m}=-25+1.71 \mathrm{j}\right)$ and titanium dioxide $\left(\mathrm{TiO}_{2}, \varepsilon d=6.38\right)$. The $\mathrm{Cy} 7$ molecules are embedded in a PMMA film deposited on the top of the HMM. The design wavelength is $773 \mathrm{~nm}$.

Following the homogenization procedure proposed in chapter 2, we obtain the HMM dispersion curves for TE and TM polarizations shown in Figure 3.3 (a) and (b), respectively. The real part (blue) of the dispersion curve for TE polarized waves is close to zero, while the imaginary part (green) is high and increases with $k_{x} / k_{0}$ (essentially a metal-like behavior). In contrast, TM polarized waves have the real part (blue) of their dispersion curve with a hyperbolic profile, as desired, in the range $2.75<\left|k_{x} / k_{0}\right|<11$. In this region, the imaginary part is close to zero allowing low loss wave propagation. Above the upper cut-off $\left(\left|k_{x} / k_{0}\right| \geq 11\right)$ the real part becomes flat and the imaginary part becomes high preventing waves from propagating in this region. Once a hyperbolic dispersion profile has been obtained, the next step is to calculate $P$ and $W_{\text {rad }}$ assuming Cy7 embedded in a PMMA layer of finite thickness.

It is worth pointing out that the previous model, defined by equations (2.26)-(2.33), only works if the QE is placed in a semi-infinite medium. This new approach, in contrast,
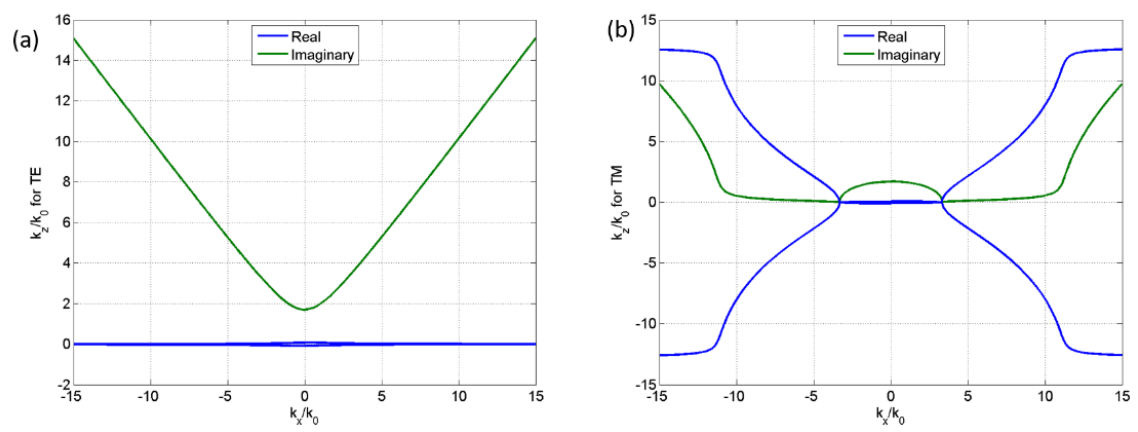

Figure 3.3. Real (blue) and imaginary (green) parts of the HMM dispersion curve for (a) TE and (b) TM polarizations 


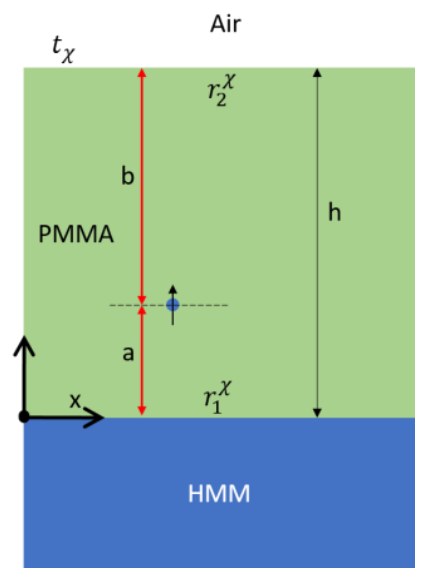

Figure 3.4. Proposed structure to model the radiation parameters of QE embedded in a PMMA layer of finite thickness.

allows the QEs to be embedded in a host layer of arbitrary thickness $h$ bounded between the HMM and air. This finite thickness causes the waves to experience multiple reflections both in the top and bottom host interfaces, which need to be accounted for by the formalism. To do so, we consider the setup shown in Figure 3.4, where the dipole is positioned at $a$ distance from the host/HMM interface and $b$ from the host/air interface $(a+b=h)$. When the host thickness is taken into account, the normalized dissipated power density of perpendicularly and parallel oriented QE is calculated, respectively, as [121], [150]:

$$
\begin{gathered}
\frac{\mathrm{d} W_{\perp}}{\mathrm{d} k_{x}}=\operatorname{Re}\left\{\frac{3}{2} \frac{1}{k_{z}}\left(\frac{k_{x}}{\sqrt{\varepsilon_{h}} k_{0}}\right)^{3}\left(1-R_{p}\right)\right\}, \\
\frac{\mathrm{d} W_{\|}}{\mathrm{d} k_{x}}=\operatorname{Re}\left\{\frac{3}{4} \frac{1}{k_{z}} \frac{k_{x}}{\sqrt{\varepsilon_{h}} k_{0}}\left[1+R_{e}+\frac{k_{z}^{2}}{\varepsilon_{h} k_{0}^{2}}\left(1-R_{p}\right)\right]\right\},
\end{gathered}
$$

where $k_{z}$ refers to the z-component of the wavevector inside the host, with $R_{p}$ and $R_{s}$ calculated as follows,

$$
R_{\chi}=\frac{r_{1}^{\chi} \mathrm{e}^{2 \mathrm{j} k_{z} a}+r_{2}^{\chi} \mathrm{e}^{2 \mathrm{j} k_{z} b}+2 r_{1}^{\chi} r_{2}^{\chi} \mathrm{e}^{2 \mathrm{j} k_{z} h}}{1-r_{1}^{\chi} r_{2}^{\chi} \mathrm{e}^{2 \mathrm{j} k_{z} h}},
$$

where $r_{n}^{\chi}$ is the reflection coefficient assuming a single interface, i.e., host/HMM $(n=1)$ or the host/air $(n=2)$, and $\chi$ is the wave polarization ( $\chi=p$ or $s$ for TM and TE polarization, respectively). The Purcell factor of a perpendicularly or parallel oriented QE $\left(P_{\alpha}\right)$ is calculated by integrating (3.1) and (3.2) as in (2.29)-(2.32). The calculation of $\eta$ requires the knowledge of the total power radiated into the far field (in the air). Thus, we start by 
writing the magnitude of the 2-dimensional (2D) Fourier transformed electric field vector radiated by a dipole perpendicularly $\left(E_{\perp}^{\chi}\right)$ and parallel $\left(E_{\|}^{\chi}\right)$ polarized as [124],

$$
\begin{gathered}
E_{\perp}^{\xi, p}=-\mathrm{j} \xi \frac{\omega^{2} \mu_{h}}{4 \pi} \frac{\sqrt{k_{x}^{2}+k_{y}^{2}}}{k_{h} k_{z}} p \mathrm{e}^{ \pm \mathrm{j} \mathrm{k}_{z} z} \\
E_{\perp}^{\xi, s}=0 \\
E_{\|}^{\xi, p}=\mathrm{j} \frac{\omega^{2} \mu_{h}}{4 \pi} \frac{k_{x}}{k_{h} \sqrt{k_{x}^{2}+k_{y}^{2}}} p \mathrm{e}^{\xi \mathrm{jk}_{z} z} \\
E_{\|}^{\xi, s}=\mathrm{j} \frac{\omega^{2} \mu_{h}}{4 \pi} \frac{k_{y}}{k_{z} \sqrt{k_{x}^{2}+k_{y}^{2}}} p \mathrm{e}^{\mathrm{j}^{\xi \mathrm{j}_{z} z}}
\end{gathered}
$$

where $p$ is the dipole momentum, $\omega$ is the angular frequency, $\mu_{h}$ is the host magnetic permeability, $k_{h}$ is the wavenumber inside the host and $\xi=1$ or -1 for wave propagating in $\mathbf{z}$ or $\mathbf{- z}$ direction, respectively. The second step consists in computing the FT of the electric field that are transmitted into the air $\left(A_{\alpha}^{\chi}\right)$. For this calculation, we need to consider the multiple reflections of $E_{\alpha}^{\chi}(\alpha=\perp$ or $\|$ for perpendicular or parallel polarized QE) at the top and bottom interfaces. Therefore, $A_{\alpha}^{\chi}$ is calculated as follows,

$$
\begin{gathered}
A_{\alpha}^{\chi}=t_{\chi}\left\{E_{\alpha}^{+, \chi} \mathrm{e}^{\mathrm{j} \mathrm{k}_{z} b}\left[1+r_{1}^{\chi} r_{2}^{\chi} \mathrm{e}^{\xi j 2 \mathrm{k}_{z} h}+\left(r_{1}^{\chi} r_{2}^{\chi} \mathrm{e}^{\xi j 2 \mathrm{k}_{z} h}\right)^{2}+\left(r_{1}^{\chi} r_{2}^{\chi} \mathrm{e}^{\xi j 2 \mathrm{k}_{z} h}\right)^{3}+\ldots\right]+\right. \\
\left.E_{\alpha}^{-, \chi} \mathrm{e}^{\mathrm{j} \mathrm{k}_{z}(h+a)}\left[1+r_{1}^{\chi} r_{2}^{\chi} \mathrm{e}^{\xi j 2 \mathrm{k}_{z} h}+\left(r_{1}^{\chi} r_{2}^{\chi} \mathrm{e}^{\xi j 2 \mathrm{k}_{z} h}\right)^{2}+\left(r_{1}^{\chi} r_{2}^{\chi} \mathrm{e}^{\xi j 2 \mathrm{k}_{z} h}\right)^{3}+\ldots\right]\right\}, \\
A_{\alpha}^{\chi}=t_{\chi}\left\{\left[E_{\alpha}^{+, \chi} \mathrm{e}^{\mathrm{j} \mathrm{k}_{z} b}+E_{\alpha}^{-, \chi} \mathrm{e}^{\mathrm{j} \mathrm{k}_{z}(h+a)}\right] \sum_{i=0}^{\infty}\left(r_{1}^{\chi} r_{2}^{\chi} \mathrm{e}^{\xi j 2 \mathrm{k}_{z} h}\right)^{i}\right\}, \\
A_{\alpha}^{\chi}=t_{\chi} \frac{\left[E_{\alpha}^{+, \chi} \mathrm{e}^{\mathrm{j} \mathrm{k}_{z} b}+E_{\alpha}^{-, \chi} \mathrm{e}^{\mathrm{j} \mathrm{k}_{z}(h+a)}\right]}{1-r_{1}^{\chi} r_{2}^{\chi} \mathrm{e}^{\xi j 2 \mathrm{k}_{z} h}},
\end{gathered}
$$

where $t_{\chi}$ is the transmission coefficient for $\chi$-polarized waves calculated at the interface host/air. Next, we use the stationary phase method [109] to propagate the Fourier transformed electric field $\left(A_{\alpha}^{\chi}\right)$ into the far field $\left(\mathbf{E}_{\boldsymbol{\alpha}}^{\mathbf{F F}}\right)$,

$$
\mathbf{E}_{\alpha}^{\mathrm{FF}}(r, \theta, \varphi)=-\mathrm{j} k_{z 0}\left[A_{\alpha}^{p} \boldsymbol{\theta}+A_{\alpha}^{s} \boldsymbol{\varphi}\right] \frac{e^{\mathrm{j} k_{0} r}}{r}=-\mathrm{j} k_{0} \cos \theta\left[A_{\alpha}^{p} \boldsymbol{\theta}+A_{\alpha}^{s} \varphi\right] \frac{e^{\mathrm{j} k_{0} r}}{r}
$$

where $r, \theta$ and $\varphi$ are the components of $\mathbf{r}$ in spherical coordinates, $\boldsymbol{\theta}$ and $\boldsymbol{\varphi}$ are unitary vectors in spherical coordinates along $\theta$ and $\varphi$ directions, respectively, and $k_{z 0}$ is the $\mathrm{z}$ component of the wavevector in free space. For the far field equation to be used in (3.11), the following transformation is required: 


$$
\mathbf{k}_{\mathbf{h}}=\left[\begin{array}{l}
k_{x} \\
k_{y} \\
k_{z}
\end{array}\right]=k_{h}\left[\begin{array}{c}
\sin \theta \cos \varphi \\
\sin \theta \sin \varphi \\
\cos \theta
\end{array}\right] \begin{aligned}
& 0 \leq \theta \leq \pi / 2 \\
& 0 \leq \varphi \leq 2 \pi
\end{aligned} .
$$

With $\mathbf{E}_{\boldsymbol{\alpha}}^{\mathbf{F F}}$ known, the time-averaged Poynting vector $\left(\left\langle\boldsymbol{S}_{\boldsymbol{\alpha}}(\mathbf{r})\right\rangle\right)$ is calculated as follows,

$$
\left\langle\mathbf{S}_{\alpha}(\mathbf{r})\right\rangle=\frac{1}{2 Z_{0}} \operatorname{Re}\left\{\mathbf{E}_{\alpha}^{\mathrm{FF}} \cdot \mathbf{E}_{\alpha}^{\mathrm{FF}}\right\} \mathbf{r}=\frac{k_{0}^{2} \cos ^{2} \theta}{2 Z_{0} r^{2}}\left[\left|A_{\alpha}^{p}\right|^{2}+\left|A_{\alpha}^{s}\right|^{2}\right] \mathbf{r},
$$

where $Z_{0}$ is the air impedance. The $\mathrm{QE}$ total radiated power into the far field $\left(Q_{\alpha}\right)$ is calculated integrating $\left\langle\mathbf{S}_{\boldsymbol{\alpha}}\right\rangle$ over the upper semi-sphere, i.e.,

$$
Q_{\alpha}=\int_{0}^{\pi / 2} \int_{0}^{2 \pi}\left\langle\mathbf{S}_{\alpha}\right\rangle \cdot \mathbf{r} \sin \theta r^{2} \mathrm{~d} \varphi \mathrm{d} \theta=\frac{k_{0}^{2}}{2 Z_{0}} \int_{0}^{\pi / 2} \int_{0}^{2 \pi} \cos ^{2} \theta \sin \theta\left[\left|A_{\alpha}^{p}\right|^{2}+\left|A_{\alpha}^{s}\right|^{2}\right] \mathrm{d} \varphi \mathrm{d} \theta .
$$

The radiation efficiency $\eta_{\alpha}$ is then finally obtained with the following expression,

$$
\eta_{\alpha}=\frac{Q_{\alpha} / W_{0}}{P_{\alpha}}
$$

where $W_{0}=p_{z}^{2} \omega k_{0}^{3} / 12 \pi \epsilon_{0}$ is the power that the dipole would radiate in free space.

\subsection{Single Cy7 molecule embedded in a PMMA layer}

The system proposed in Figure 3.2 is now simulated with the full-wave 3D Lumerical FDTD [151] so that the electromagnetic behavior of QEs embedded in the PMMA host layer can be assessed. Assuming $a=10 \mathrm{~nm}$, we obtain the normalized amplitude of the $\mathrm{x}$ and z-components of the electric field as shown in Figure $3.5(\mathrm{a}, \mathrm{c})$ and $(\mathrm{b}, \mathrm{d})$, respectively, for a QE perpendicularly $(a, b)$ and parallel $(c, d)$ polarized. For comparison sake, the dipole moment $p$ for both polarizations are equally set and the electric fields shown in Figure 3.5 (a-d) are normalized by their maximum value. The dashed lines in Figure 3.5 (a-d) represent the spatial region where the HMM is localized. Note that for both, perpendicular and parallel polarizations, the power dissipated by the QE is mostly coupled into the HMM, as can be concluded by the high intensity of the electric field inside it, as shown in Figure 3.5 (a-d). This figure also shows that the power propagating into the far field is weaker when compared with the power inside the HMM which, according to (3.15), implies in low external quantum efficiency $\left(\eta_{\alpha}\right)$. This behavior is more evident for the perpendicular polarization $(a, b)$ due its weaker electric field amplitude in air when compared to the parallel polarization $(c, d)$. This is explained by the destructive and constructive interference between the QE radiated wave $\left(k_{x} \leq k_{0}\right)$ and its multiple reflections at the HMM surface for perpendicular and parallel polarizations, respectively, when $h<<\lambda$. Consequently, $\eta_{\|}>\eta_{\perp}$ in this scenario. Figure 3.5 (a-d) provides a qualitative method to infer on both the QE's power dissipation and $\eta$. Quantitatively, the power 


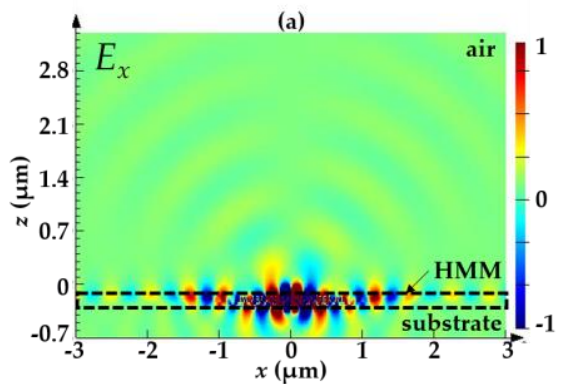

(c)

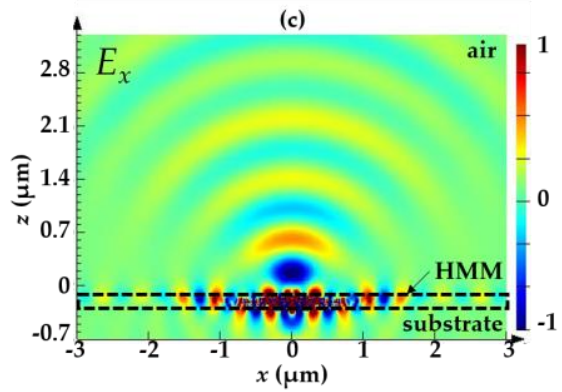

(b)

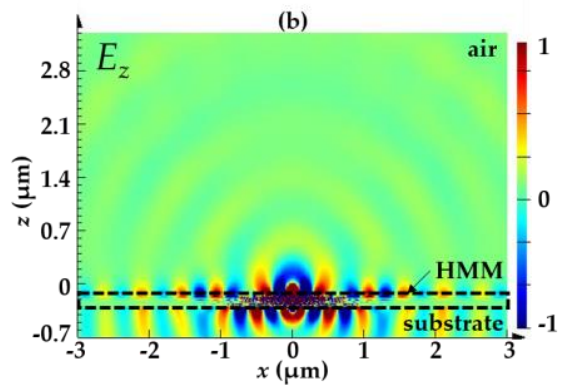

(d)

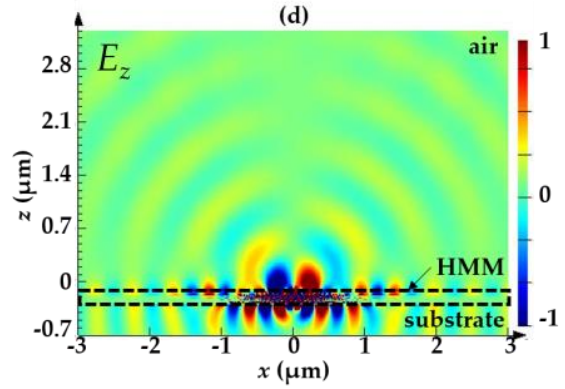

Figure 3.5. Normalized $x-(a, c)$ and $z-(b, d)$ electric field components of a dipole perpendicularly $(a, b)$ and parallel $(c, d)$ polarized. The HMM is highlighted in (a-d) with black dashed lines.

dissipated by the QE can be calculated using the normalized dissipated power density $\left(\mathrm{d} W_{\alpha} / \mathrm{d} k_{x}\right)$, from (3.1)-(3.3). Figure 3.7 (a) shows $k_{0} \mathrm{~d} W_{\alpha} / \mathrm{d} k_{x}$ for perpendicularly (blue) and parallel (red) polarized QEs. Figure 3.7 (b) shows a zoom-in of the marked region in Figure 3.7 (a), which highlights the region where the power is effectively radiated into the far field (gray area $k_{x} / k_{0}<1$ ). This figure also shows that most of the power dissipated by the QE is inside the evanescent region $\left(k_{x} / k_{0}>1\right)$. Moreover, it is possible to resolve five peaks where the QE dissipates more power, namely, $k_{x} / k_{0}=2,6,7.1,8.3$ and 10, corresponding to the high- $k$ modes supported by the HMM. The Purcell factor for perpendicular and parallel polarizations are obtained by integrating both curves in Figure 3.7 (a), resulting in $P_{\perp}=156$ and $P_{\|}=72$. From these high Purcell factors, it is possible to conclude that the HMM proposed in Figure 3.2 indeed increases the power dissipated by the QE (Cy7), consequently increasing its spontaneous emission rate. Nevertheless, the portion of the total power that effectively propagates into the far field $\left(k_{x} / k_{0}<1\right)$ is very small, as shown in Figure $3.7(\mathrm{~b})$. This power is calculated with the help of (3.14), resulting in $Q_{\perp} / W_{0}=0.0875$ and $Q_{\|} / W_{0}=0.3068$. These results are consistent with the behavior observed in Figure 3.5 (a-d) which shows that the radiated field is stronger for the parallel polarization. Therefore, the HMM increases the total power dissipated by a perpendicular or parallel polarized QE by a factor of 156 and 72, respectively. Nonetheless, only $0.05 \%$ and $0.42 \%$ of the total dissipated power, respective to perpendicular and parallel polarization, are indeed converted into photons that can propagate to the far field in the air. 


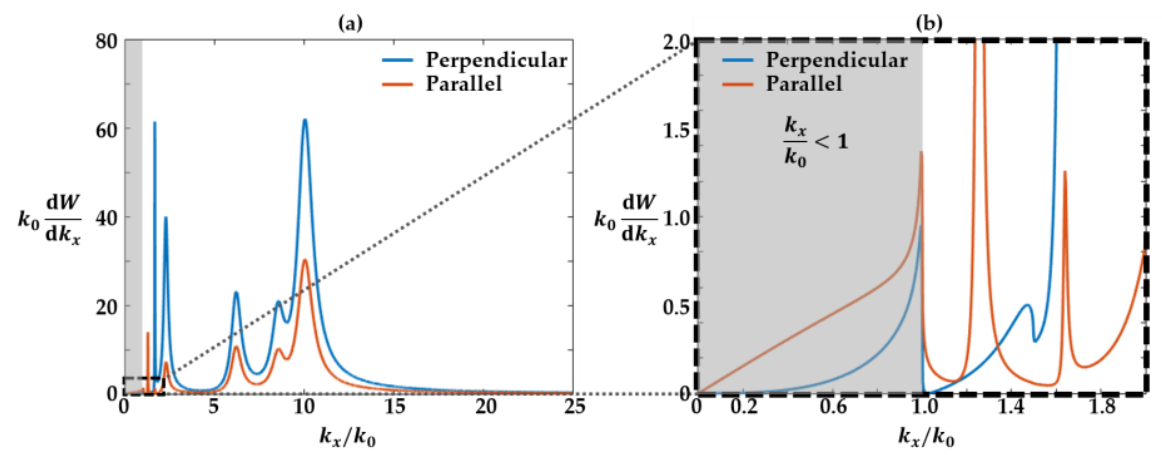

Figure 3.7. Normalized dissipated power density $\left(k_{0} \mathrm{~d} W_{\alpha} / \mathrm{d} k_{x}\right)$ for perpendicular (blue) and parallel (red) polarized QEs for (a) $0 \leq k_{x} / k_{0} \leq 25$ and (b) $0 \leq k_{x} / k_{0} \leq 2$, respectively. The gray area represents the propagation region $\left(k_{x} / k_{0} \leq 1\right)$.

\subsection{Homogenous distribution of Cy7 inside the PMMA layer}

In section 3.2, we have considered the QE fixed at $a=10 \mathrm{~nm}$ to verify if the HMM proposed in Figure 3.2 can enhance the spontaneous emission rate of Cy7. However, in most applications, QEs are randomly distributed and polarized in the host PMMA layer, as shown Figure 3.6. Moreover, the decay rate, the Purcell factor and the quantum efficiency depend on the contribution of each QE embedded in the host layer. Consequently, it is important to calculate $\eta_{\alpha}$ and $P_{\alpha}$ for all possible positions, which in this system can be accomplished by calculating both parameters, $\eta_{\alpha}(a)$ and $P_{\alpha}(a)$, as function of $a \in[0, h]$. Considering the QEs uniformly distributed along the z-axis of the host PMMA layer (see Figure 3.2), the normalized time dependent decay rate, $g(t)$, can be calculated as follows [109], [114]

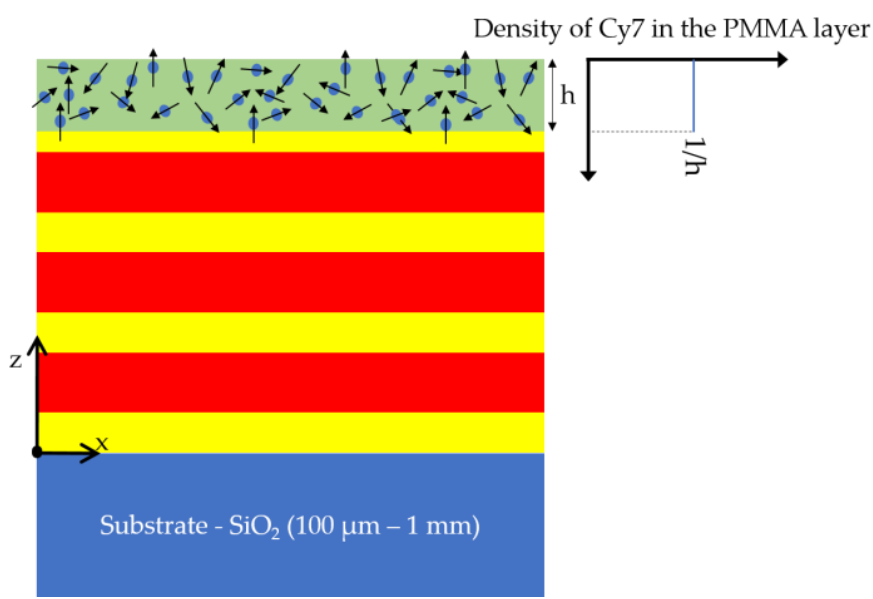

Figure 3.6. QE uniformly distributed along the PMMA layer. 


$$
\begin{gathered}
g(t)=\int_{0}^{h} \frac{\left(\Upsilon_{x} \eta_{x}(a) e^{-P_{x}(a) t}+\Upsilon_{z} \eta_{z}(a) e^{-P_{z}(a) t}\right)}{h \eta_{e f f}} \mathrm{~d} a, \\
\eta_{\text {eff }}=\int_{0}^{h} \frac{\left(\Upsilon_{x} \eta_{x}(a)+\Upsilon_{z} \eta_{z}(a)\right)}{h} \mathrm{~d} a,
\end{gathered}
$$

where $\eta_{\text {eff }}$ is the system's quantum efficiency and $\Upsilon_{\alpha}$ is the probability of the QE being polarized along the $\alpha$-axis. Note that, for mathematical consistence, the sum of the probabilities must be 1, i.e., $\Upsilon_{x}+\Upsilon_{z}=1$. In (3.16), the normalized time dependent decay $(g(t))$ is weighted by the QE's quantum efficiency in each position. After calculating $g(t)$, the system's lifetime $(\tau)$ is estimated as $g(\tau)=1 / \mathrm{e}$, resulting in the effective enhancement of spontaneous emission rate $P_{\text {eff }}=1 / \tau$.

\subsubsection{Purcell factor and radiated power}

The proposed set of equations (3.1)-(3.14) is now applied to calculate the Purcell factor $\left(P_{\alpha}\right)$ and the normalized radiated power $\left(Q_{\alpha} / W_{0}\right)$ of the system proposed in Figure 3.2. Both parameters, $P_{\alpha}$ and $Q_{\alpha}$, are calculated as function of the distance between the $Q E$ and the HMM surface (a) for perpendicular (blue) and parallel (red) polarization, as shown in Figure 3.8 (a) and (b), respectively. Note that $P_{\alpha}$ increases exponentially when QE approaches the HMM surface due to the higher coupling of the evanescent fields with the structure. Differently from parallel polarized QEs, which radiate both TE and TM polarized waves ((3.6) and (3.7)), perpendicularly polarized QEs radiate only TM polarized waves ((3.4) and (3.5)). Since the HMM is designed to have a hyperbolic dispersion curve only for TM polarization (the stack behaves as metal for TE polarization, as seen in Figure 3.3), $P_{\perp}$ is higher than $P_{\|}$, as shown in Figure 3.8 (a). The opposite is observed for $Q_{\alpha} / W_{0}$, where the radiated power is higher for parallel polarized QEs. This is explained by two main factors. First, the destructive and constructive interference

(a)

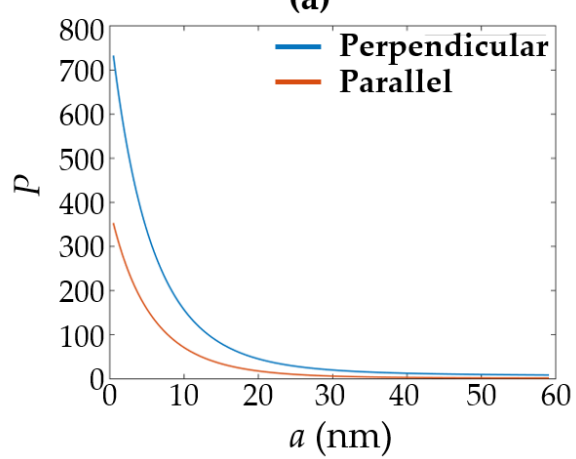

(b)

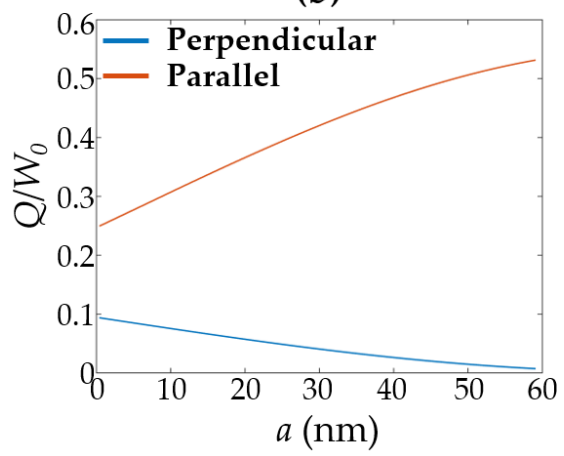

Figure 3.8. (a) Purcell factor $\left(P_{\alpha}\right)$ and (b) normalized radiated power $\left(Q_{\alpha} / W_{0}\right)$ of the system proposed in Figure 3.2 calculated as function of the distance between the QE and the HMM surface (a) for perpendicular (blue) and parallel (red) polarizations. 
between the wave radiated by the QE $\left(\sqrt{k_{x}^{2}+k_{y}^{2}} \leq k_{h}\right)$ and its reflection at the HMM surface for perpendicular and parallel polarizations, respectively, when $h<<\lambda$. Second, the parallel polarized QEs have most of their power in the region $\sqrt{k_{x}^{2}+k_{y}^{2}} \leq k_{0}$, which propagates in air, while perpendicularly polarized QEs have most of their power in the region $k_{0} \leq \sqrt{k_{x}^{2}+k_{y}^{2}} \leq k_{h}$, mostly reflected at the host/air interface.

Close to the HMM surface $(a<2 \mathrm{~nm}), P_{\perp}$ and $P_{\|}$reach values close to 700 and 350, respectively, as seen in Figure 3.8 (a). Although these values represent a high Purcell factor, according to Figure $3.8(\mathrm{~b})$ at these points $Q_{\perp} / W_{0}$ and $Q_{\|} / W_{0}$ are 0.1 and 0.25 , respectively, implying in quantum efficiencies as low as $0.014 \%$ and $0.07 \%$ for perpendicular and parallel polarizations, respectively. When the QEs are close to PMMA/air interface $(a>50 \mathrm{~nm})$, the evanescent field emitted by them reaches the HMM surface with less energy, resulting in $P_{\perp}$ and $P_{\|}$lower than 9.9 and 2.23, respectively, but with $\eta$ as high as $0.15 \%$ and $22.7 \%$ for perpendicular and parallel polarization, respectively.

\subsubsection{Radiation pattern}

The low $Q_{\alpha}$ for both perpendicular and parallel polarizations make the use of photon counters (PC) to measure $g(t)$ a challenging task. Furthermore, it is important to understand the QEs radiation pattern so that the PCs can be placed in the position where the radiation is maximum. Thus, we calculate the modulus of the time averaged Poynting vector for perpendicular $\left(\left\langle\mathbf{S}_{\mathbf{z}}\right\rangle\right)$ and parallel $\left(\left\langle\mathbf{S}_{\mathbf{x}}\right\rangle\right)$ polarizations as function of $a$ and $\theta$ with the help of (3.13). In this equation, the dipole moment $p$ is chosen to guarantee a dipole radiation of $1 \mathrm{~W}$ in free space. $\left\langle\mathbf{S}_{\mathbf{x}}\right\rangle$ and $\left\langle\mathbf{S}_{\mathbf{z}}\right\rangle$ are shown in Figure $3.9(\mathrm{a})$ and (b), respectively. Figure 3.9 (a) shows that for a perpendicular polarized $\mathrm{QE}$, the maximum emission occurs close to the HMM (as explained in Figure $3.8(\mathrm{a})$ ) and at an angle $\theta=50^{\circ}$. In contrast, the maximum emission for a parallel polarized QE occurs close to the host/air interface, and with the emission peak normal to the HMM surface $\left(\theta=0^{\circ}\right)$. The knowledge of the emission peak also helps to calculate the optimum objective aperture angle to maximize photon collection. Another interesting behavior observed from these results is
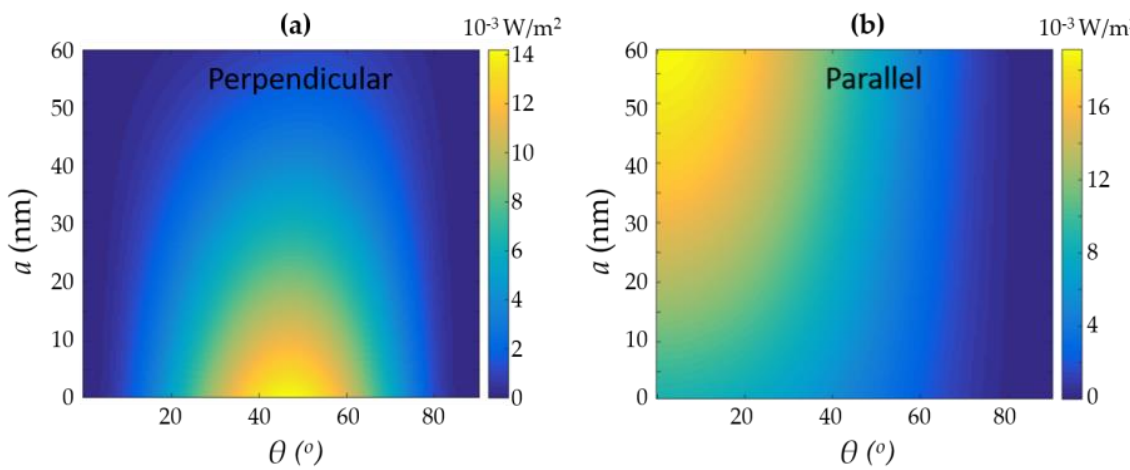

Figure 3.9. Radiation pattern as function of the distance for (a) perpendicular and (b) parallel polarized QEs. 
that perpendicular and parallel polarizations have different maximum emission angles. This behavior is important if the radiation parameters of each individually polarization is of interest. Therefore, a PC can be placed at $\theta=0^{\circ}$ or $50^{\circ}$ for perpendicular or parallel polarized QEs, respectively.

\subsubsection{Decay curve}

With the knowledge of $P_{\alpha}$ and $Q_{\alpha}$ as function of distance (a), the decay curve $g(t)$ and the system's quantum efficiency $\eta_{\text {eff }}$ can be calculated with (3.16) and (3.17), respectively. However, the QEs polarization probability (perpendicular or parallel) $\left(\Upsilon_{\alpha}\right)$ is required in both equations. In real cases, we cannot predict the exact behavior of the system since the QEs are randomly polarized inside the PMMA layer.

Alternatively, we can consider three different scenarios: in the first and second scenarios the QEs are fully perpendicularly $\left(\Upsilon_{\perp}=1, \Upsilon_{\|}=0\right)$ and parallel $\left(\Upsilon_{\perp}=0, \Upsilon_{\|}=1\right)$ polarized, respectively, while in the third the QEs are equally polarized $\left(\Upsilon_{\perp}=1 / 3, \Upsilon_{\|}=2 / 3\right)$. Note that in the third scenario $\Upsilon_{\|}=2 \Upsilon_{\perp}$, since parallel polarization requires the QEs to be aligned along the $x$ - and $y$-axes. In contrast, perpendicular polarization requires the QEs to be aligned along the $\mathrm{z}$-axis only. Furthermore, in real cases, $\Upsilon_{\perp}$ and $\Upsilon_{\|}$are unknown and limited to the interval $[0,1]$. Thus, fully perpendicular or parallel polarized QEs are limiting scenarios that define a region of $g(t)$ where QEs are randomly distributed. This region is defined from hereon as the decay region.

The decay behavior for the three scenarios, perpendicular (blue line), parallel (green line) and equally polarized (dashed red lines), are shown in Figure 3.10. The gray area in this figure represents the decay region and the x-axis is normalized by the QE's lifetime $\left(\tau_{0}\right)$.

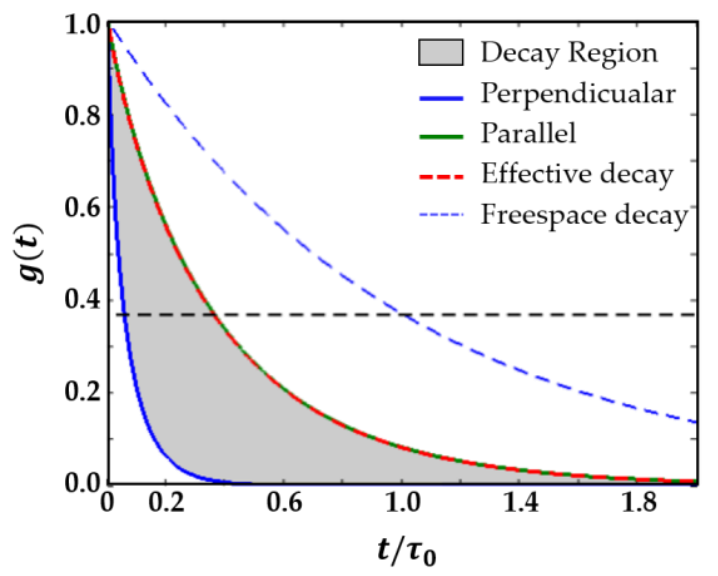

Figure 3.10. Decay behaviour of the quantum emitter considering $\left[\Upsilon_{\perp}, \Upsilon_{\|}\right]$equals to [1,0] (blue), $[0,1]$ (red) and $[1 / 3,2 / 3]$ (dashed red lines). The gray area represents is the decay region and the QE's decay behavior in free space are shown as dashed light blue lines). To facilitate the $P_{\alpha}$ calculation, we also show $g(t)=1 / \mathrm{e}$ as black dashed line. Note that the $\mathrm{x}$-axis is normalized by $\tau$. 
Table 3.1. System decay parameters $\left(\tau / \tau_{0}, P_{e f f}\right.$ and $\left.\eta_{e f f}\right)$ for perpendicular, parallel and equally polarized QEs.

\begin{tabular}{rccc} 
& $\boldsymbol{\tau} / \boldsymbol{\tau}_{\mathbf{0}}$ & $\boldsymbol{P}_{\text {eff }}$ & $\boldsymbol{\eta}_{\text {eff }}(\mathbf{\%})$ \\
\cline { 2 - 4 } Perpendicular & 0.053 & 17.73 & 0.131 \\
Parallel & 0.357 & 2.800 & 10.00 \\
Equally polarized & 0.358 & 2.790 & 6.778
\end{tabular}

The free space decay behavior (dashed light blue line) is also plotted for comparison sake. This figure also shows that the HMM decreases the system's lifetime for both perpendicular $\left(\tau_{\perp}=0.053 \tau_{0}\right)$ and parallel $\left(\tau_{\|}=0.357 \tau_{0}\right)$ polarizations by a factor of 17.53 $\left(P_{e f f}^{\perp}\right)$ and $2.8\left(P_{e f f}^{\perp}\right)$, respectively, when compared to the free space lifetime. Moreover, it shows that perpendicularly polarized QEs decay faster when compared to parallel polarized $\left(P_{\text {eff }}^{\perp}>P_{\text {eff }}^{\perp}\right)$ ones because, according to Figure $3.8(\mathrm{a}), P_{\perp}(a)>P_{\|}(a) \forall a$. In contrast, $\eta_{\alpha}(a)$ is higher for parallel polarization, especially for QEs located farther away from the HMM surface, as seen in Figure 3.8 (b). Combining these properties, along with the fact that $g(t)$ is weighted by $\eta_{\alpha}$ and $\Upsilon_{\alpha}$, we obtain that the decay behavior for $\Upsilon_{\perp}=1 / 3$ and $\Upsilon_{\|}=2 / 3$ is governed mainly by the parallel polarization, as seen in Erro! Fonte de referência não encontrada.. The pertinent system parameters for all three scenarios are summarized in Table 3.1. According to this table, the effective quantum efficiency of perpendicularly polarized QEs $\eta_{\text {eff }}(0.131 \%)$ is 76.33 times lower than that for parallel polarized QEs $\left(\eta_{\text {eff }}=10 \%\right)$. Therefore, for the calculation of $g(t), 76$ perpendicularly polarized QEs have the same effect as 1 parallel QE, according to (3.16). The huge $\eta_{\text {eff }}$ difference for both polarizations explains why $g(t)$ for the equally polarized QEs is closer to the parallel polarization, as seen in Figure 3.10. In addition, for equally polarized QEs, $\eta_{\text {eff }}=6.77 \%$, which is basically $2 / 3$ the $\eta_{\text {eff }}$ of parallel polarized QEs since $1 / 3$ of the QEs are perpendicularly polarized and do not contribute significantly for increasing the system's quantum efficiency.

The decay curve for $h=60 \mathrm{~nm}$ show a little enhancement of $\Gamma(2.79)$, with a large reduction of $\eta_{\text {eff. }}$ As seen in Figure 3.8, $P_{\alpha}$ and $\eta_{\alpha}$ are highly dependent on the distance from the HMM surface. Thus, changing $h$ would change significantly the system's response, making it possible control $g(t)$ by adjusting $h$. In the next section we investigate the influence of $h$ on $\eta_{\text {eff }}$ and $P_{\text {eff. }}$

\subsection{Influence of the PMMA thickness in system's Purcell factor and quantum efficiency}

As previously mentioned, the host PMMA layer is deposited on the top of the HMM via spin-coating technique. According Microchem 950PMMA A Resists A2 datasheet, it is possible to control $h$ by the right choice properly adjusting the spin-coating velocity. The minimum (maximum) possible $h$ achieved by this technique is $60 \mathrm{~nm}(100 \mathrm{~nm})$, which is accomplished by setting the spin-coating velocity to 5000rpm (1000rpm). Nevertheless, as discussed in the last section, the HMM does contribute significantly to increasing $\Gamma$ of 

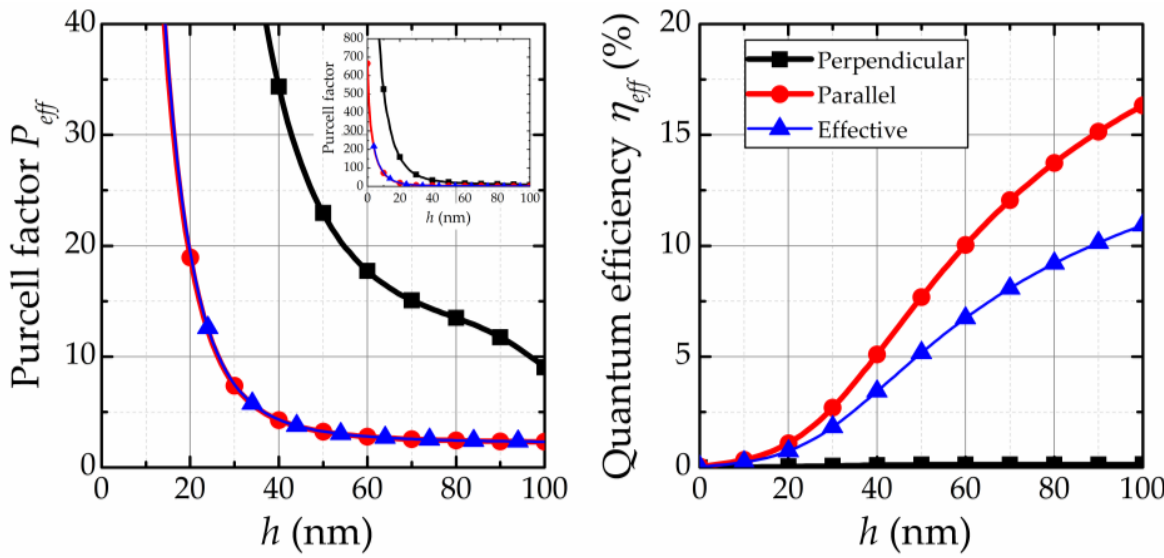

Figure 3.11. (a) $P_{\text {eff }}$ and (b) $\eta_{\text {eff }}$ as function of $h$ for three scenarios: QEs fully perpendicularly (squares) and parallel (circles) polarized, and QEs equally polarized (triangles). In (a) $P_{\text {eff }}$ is limited to 40 for better visualization, the full curve is shown in the inset.

Cy7 molecules for $h=60$. Since $P_{\alpha}$ is higher close to the HMM surface, one way of increasing $P_{\text {eff }}$ is by making $h<60 \mathrm{~nm}$. This can be realized by mixing the PMMA with a solvent (such as ethanol). Since the solvent viscosity is lower than the PMMA's, by changing the PMMA concentration and adjusting the spin-coating velocity it is possible to achieve $h<60 \mathrm{~nm}$. The procedure to control the PMMA thickness is described in Appendix B.

Next, we calculate $P_{\text {eff }}$ and $\eta_{\text {eff }}$ for the system shown in Figure 3.2 assuming $1 \mathrm{~nm} \leq h \leq$ 100nm. Figure 3.11 (a) and (b) show $P_{\text {eff }}$ and $\eta_{\text {eff, }}$ respectively, for three scenarios: in the first and second scenarios the QEs are fully perpendicularly (squares) and parallel (circles) polarized, respectively, while in the third the QEs are equally polarized (triangles). We show $P_{\text {eff }}$ values up to 40 in Figure 3.11 (a) for the sake of better visualization, while the inset shows the full curve. According to Figure 3.11 (a), $P_{\text {eff }}$ is highly enhanced for very thin layers $(h<4 \mathrm{~nm})$, reaching values up to 700 for both polarizations. Nevertheless, in this scenario $(h<4 \mathrm{~nm})$, a great part of the power coupled inside the HMM is attenuated by ohmic losses associated with high- $k$ modes, reducing the power radiated into the air. Consequently, $\eta_{\text {eff }}$ is very small $\left(\eta_{\text {eff }}<0.5 \%\right)$ for both polarizations, as seen in Figure 3.11 (b). Additionally, $\eta_{\text {eff }}$ is much smaller for perpendicular polarization than for parallel polarization for any $h$. Consequently, perpendicular QEs do not have a significant influence when the QEs are equally polarized, as explained in the last subsection. For thick layers $(h>60 \mathrm{~nm}), P_{\text {eff }}$ is lower than 3 for parallel polarization, and $\eta_{\text {eff }}$ is higher than $10 \%$. As seen in Figure 3.11 (a), it is possible to choose $P_{\text {eff }}$ from 2.5 to 700 by controlling $h$. However, the higher $P_{\text {eff }}$ the lower is $\eta_{\text {eff. }}$. Therefore, a tradeoff between $P_{\text {eff }}$ and $\eta_{\text {eff }}$ needs to be found to optimize $h$. For instance, if the design requires $P_{\text {eff }}=10, h$ should be equal to $28 \mathrm{~nm}$, resulting in $\eta_{\text {eff }}=1.25 \%$. Therefore, Figure 3.11 represents a useful tool for the design of HMMs. 
As seen in Figure 3.11, although we can achieve high $P_{\text {eff }}$ (up to 20 for $h>20 \mathrm{~nm}$ ) using the HMM proposed in Figure 3.2, $\eta_{\text {eff }}$ is very low $(<7.5 \%$ for $h<60 \mathrm{~nm})$. Therefore, some authors have proposed the use of diffraction gratings to circumvent this issue, since it converts bound SPP waves (high momenta) into propagating waves [39], [55], [57], [120][123], [126], [128], [129] (low momenta). In the next chapter we show how to optimize the geometrical parameters of the grating in order to increase $\eta_{\text {eff }}$ while maintaining a high Peff.

\subsection{Chapter conclusion}

In this chapter we have proposed an HMM capable of enhancing the Purcell factor of the fluorescent molecules (Cy7). These molecules are embedded in a thin PMMA host film deposited on the top of the HMM. This structure was modeled with a new mathematical formalism to calculate $P$ and $\eta$ taking into account the reflections at the upper and bottom host interfaces. Moreover, we have also proposed an analytical method to calculate $g(t)$ as function of the QE's position inside the host layer. We demonstrated that QEs placed far from the HMM surface have lower $P$ but higher $\eta$. Thus, they contribute more to $g(t)$ than those closer to the HMM surface. Finally, we have shown that the host thickness has a direct influence on the system's $g(t)$, and that with an adequate choice of $h$ it is possible to control the systems decay behavior. 


\section{Chapter 4}

Design and analysis of grating-assisted

radiation emission of

QE in HMM 


\section{Chapter 4 - Summary}

4.1 Chapter 4 - Introduction.................................................................................................63

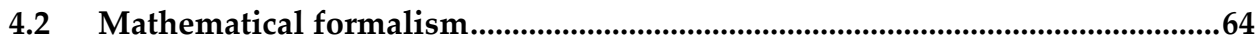

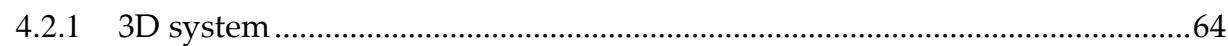

4.2.1.1 Calculation of the transmitted and reflected electric fields .....................65

4.2.1.2 Perpendicularly polarized dipole .............................................................68

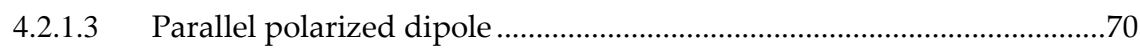

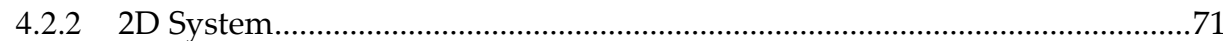

4.2.2.1 Calculation of the transmitted and reflected electric fields .....................72

4.2.2.2 Perpendicularly polarized dipole .............................................................74

4.2.2.3 Parallel polarized dipole ...........................................................................

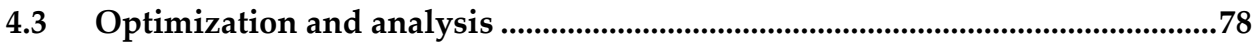

4.3.1 2D Optimization procedure ...............................................................................

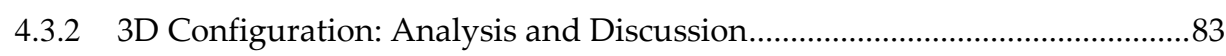

4.4 Comparison between HMM with and without metallic gratings.....................87

4.5 Computation improvement of our method ..........................................................89

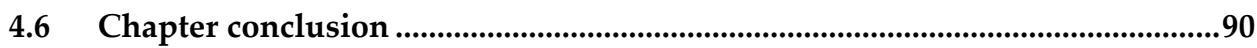




\subsection{Chapter 4 - Introduction}

In Chapter 3, we showed that although it is possible to decrease the QE lifetime using HMM, most of the power dissipated by the QEs is coupled into the HMM as surface plasmon polariton (SPP) waves. The high- $k$ states of these waves prevent them from propagating in free space [37], [38], [40], [97]-[99]. Therefore, the energy is thermally dissipated inside the HMM with a consequent reduction of the sources' external quantum yield. Some authors have proposed the use of a diffraction grating to solve this problem, since it converts bound SPP waves (high momenta) into propagating waves [33], [48], [50], [109]-[118] (low momenta). However, the authors resorted to numerical simulations (as opposed to analytical or semi-analytical simulations) for the grating design optimization that, as shown in this chapter, do not provide clear-cut insights on how the grating affects the QEs' behavior.

In this sense, we propose in chapter 4 a semi-analytical method that can be successfully applied to the design of diffraction gratings aimed at optimizing the conversion of high$k$ modes into propagating modes. In addition, this method directly provides the QE's PDoS, which is a computationally costly task if carried out numerically. Both 2D and 3D configurations are examined, with the former being used for the grating optimization procedure due to its lower computational cost. The structure is implemented in the 3D model only after the optimum grating parameters have been obtained. In addition, the proposed method facilitates the grating design, which is useful to control the QEs' emission directivity. Another advantage of this method is the reduced computational burden to obtain the Purcell factor and the radiated power as function of the QEs and grating relative position, highlighting the behavior of each QE to the system. After the optimization procedure, we show that the HMM with gratings proposed in this chapter have a better performance than the HMM showed in chapter 3.

The remainder of this Chapter is organized as follows. Section 4.1 presents the mathematical formalism. Next, we apply our optimization procedure to a metallic grating placed on top of an alternating stack of $\mathrm{Ag}$ and $\mathrm{TiO}_{2}$ layers, showed in Chapter 3. We begin the analysis with the $2 \mathrm{D}$ optimization of the grating structure, followed by the 3D simulation carried out with the optimized parameters. The figures-of-merit are the radiation pattern and the Purcell factor, both as function of the QE position. Finally, we present some concluding remarks about the results obtained in this Chapter.

Part of the work presented in this chapter has been adapted with permission from:

A. F. Mota, A. Martins, H. Ottevaere, W. Meulebroeck, E. R. Martins, J. Weiner, F. L. Teixeira, and B.-H. V. Borges, "Semianalytical model for design and analysis of gratingassisted radiation emission of quantum emitters in hyperbolic metamaterials," ACS Photonics, vol. 5, no. 5, pp. 1951-1959, 2018. Copyright 2018 American Chemical Society. The copyrights permission is found in Annex 2 of this thesis. 


\subsection{Mathematical formalism}

In this subsection we show how the equations to calculate the Purcell factor and the dipole radiated power for a three-dimensional (3D) grating are derived. Since 3D simulations are computationally costly, particularly if used in the optimization of grating parameters, we also present a simplified two-dimensional (2D) formalism for a dipole interacting with a 2D grating. This 2D method allows us not only to obtain the reflection and transmission coefficients for all diffraction orders but also to more efficiently optimize the grating parameters for controlling the dipole's radiation pattern.

\subsubsection{D system}

The geometry adopted in the 3D method is illustrated in Figure 4.1, where the quantum emitters (QE) are treated as a dipole embedded in medium 1 (with permittivity $\varepsilon_{1}$ ). The $\mathrm{QE}$ is centered at the origin at a distance $q$ from the hyperbolic metamaterial's (HMM's) bottom surface and $z_{g r}$ from the grating upper surface. The metallic grating with periodicities $\Lambda_{x}$ and $\Lambda_{y}$ in $\mathrm{x}$ and y directions, respectively, is on top of the HMM and is covered with medium 2 (with permittivity $\varepsilon_{2}$ ). In the next section, we calculate the transmitted and reflected electric fields which are required to obtain the Purcell factor and the dipole's radiated power.

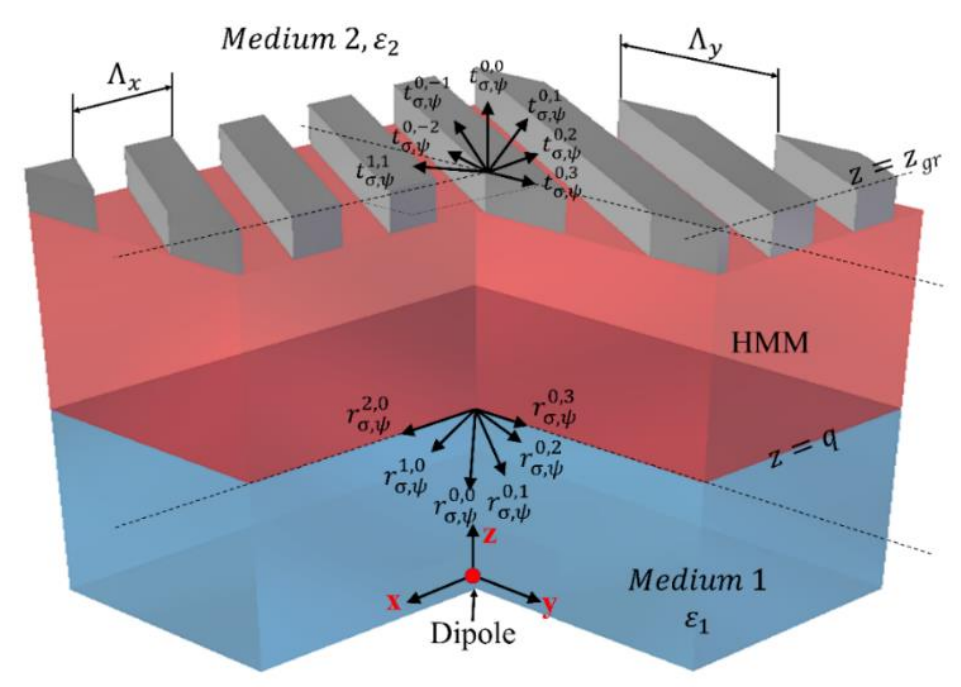

Figure 4.1. Proposed 3D geometry in which the QEs are modelled as a dipole embedded in medium 1 (with permittivity $\varepsilon 1$ ), centered at the origin and at a distance $q$ from the HMM bottom surface. The grating with periodicities $\Lambda_{x}$ and $\Lambda_{y}$ in $\mathrm{x}$ and y directions, respectively, on top of the HMM is covered with medium 2 (with permittivity $\varepsilon 2$ ). 


\subsubsection{Calculation of the transmitted and reflected electric fields}

Assuming temporal dependence $e^{-j \omega t}$, the current generated by a dipole source centered at the origin and embedded in medium $1(\mathbf{J})$ is calculated as follows [109],

$$
\mathrm{J}(\mathbf{r})=-\mathrm{j} \omega \delta^{(3)}(\mathbf{r}) \mathbf{p}
$$

where $\mathbf{r}=x \mathbf{x}+y \mathbf{y}+z \mathbf{z}, \delta^{(3)}(\mathbf{r})$ is the 3D Dirac delta function, $\mathbf{p}$ is the dipole moment and $\omega$ is the angular frequency. The electric field $\mathbf{E}_{\mathbf{i}}$ at $\mathbf{r}$ generated by $\mathbf{J}$ is then written as

$$
\mathbf{E}_{\mathbf{i}}(\mathbf{r})=\omega^{2} \mu_{1}\left(\mathbf{I}+\frac{1}{k_{1}^{2}} \nabla \nabla\right) \frac{\mathrm{e}^{\mathrm{j} k_{1}|\mathbf{r}|}}{4 \pi|\mathbf{r}|} \mathbf{p} .
$$

where $\mu_{1}$ is the permeability of medium $1, \mathbf{I}$ is the identity tensor and $k_{1}=\left|\mathbf{k}_{\mathbf{1}}\right|=$ $\left|k_{x} \mathbf{x}+k_{y} \mathbf{y}+k_{z 1} \mathbf{z}\right|$ is the wavenumber vector magnitude in medium 1. Using the Weyl identity

$$
\frac{e^{\mathrm{j} k_{1}|\mathrm{r}|}}{4 \pi|\mathbf{r}|}=\frac{1}{2 \pi} \int_{-\infty}^{\infty} \int \frac{\mathrm{j}}{4 \pi k_{z 1}} \mathrm{e}^{\mathrm{j}\left(k_{x} x+k_{y} y \pm k_{z 1}\right)} \mathrm{d} k_{x} \mathrm{~d} k_{y}
$$

in (4.2), we obtain,

$$
\mathbf{E}_{\mathbf{i}}(\mathbf{r})=\omega^{2} \mu_{1}\left(\mathbf{I}+\frac{1}{k_{1}^{2}} \nabla \nabla\right) \frac{1}{2 \pi} \int_{-\infty}^{\infty} \int \frac{\mathrm{j}}{4 \pi k_{z 1}} \mathrm{e}^{\mathrm{j}\left(k_{x} x+k_{y} y \pm k_{z 1} z\right)} \mathrm{d} k_{x} \mathrm{~d} k_{y} \mathbf{p} .
$$

The spatial differentials are more easily considered if $\mathbf{E}_{\mathbf{i}}$ is Fourier transformed (FT). The FT and the inverse FT are defined as

$$
\begin{aligned}
& \mathbf{E}_{\mathbf{i}}^{\mathrm{FT}}\left(\mathbf{k}_{\|,}, z\right)=\frac{1}{2 \pi} \int_{-\infty}^{\infty} \int \mathbf{E}_{\mathbf{i}}(\mathbf{r}) \mathrm{e}^{-\mathrm{j}\left(k_{x} x+k_{y} y\right)} \mathrm{d} x \mathrm{~d} y, \\
& \mathbf{E}_{\mathbf{i}}(\mathbf{r})=\frac{1}{2 \pi} \int_{-\infty}^{\infty} \int \mathbf{E}_{\mathbf{i}}^{\mathrm{FT}}\left(\mathbf{k}_{\| \prime}, z\right) \mathrm{e}^{\mathrm{j}\left(k_{x} x+k_{y} y\right)} \mathrm{d} k_{x} \mathrm{~d} k_{y},
\end{aligned}
$$

thus

$$
\begin{aligned}
\mathbf{E}_{\mathbf{i}}^{\mathrm{FT}}\left(\mathbf{k}_{\|}, z\right) & =\mathrm{j} \frac{\omega^{2} \mu_{1}}{4 \pi} \frac{1}{k_{1}^{2} k_{z 1}}\left[\begin{array}{ccc}
k_{1}^{2}-k_{x}^{2} & -k_{x} k_{y} & \mp k_{x} k_{z 1} \\
-k_{x} k_{y} & k_{1}^{2}-k_{y}^{2} & \mp k_{y} k_{z 1} \\
\mp k_{x} k_{z 1} & \mp k_{y} k_{z 1} & k_{1}^{2}-k_{z 1}^{2}
\end{array}\right] \mathbf{p} \mathrm{e}^{\xi j k_{z 1} z} \\
& =\mathrm{j} \frac{\omega^{2} \mu_{1}}{4 \pi} \mathbf{M}\left(\mathbf{k}_{\|}, z\right) \mathbf{p},
\end{aligned}
$$

where $\mathbf{k}_{\|}=k_{x} \mathbf{x}+k_{y} \mathbf{y}$ is the parallel component of $\mathbf{k}_{1}$ and $\xi=1$ or -1 for wave propagating in $\mathbf{z}$ or $\mathbf{- z}$ direction, respectively. The electric field can next be written as a sum of two orthogonal polarizations, viz. transverse electric (TE or $s$ ) and transverse magnetic (TM 
or $p$ ). For the electric field to be decomposed, $\mathbf{M}$ must be split into two terms, $\mathbf{M}^{\mathrm{s}}$ (spolarization) and $\mathbf{M}^{\mathrm{p}}$ (p-polarization), as follows

$$
\begin{gathered}
\mathbf{M}\left(\mathbf{k}_{\|}, z\right)=\mathbf{M}^{\mathrm{s}}\left(\mathbf{k}_{\| \prime}, z\right)+\mathbf{M}^{\mathrm{p}}\left(\mathbf{k}_{\|,}, z\right), \\
\mathbf{M}^{\mathrm{s}}\left(\mathbf{k}_{\| \mid}, z\right)=\frac{\mathrm{e}^{\xi k_{z 1} z}}{k_{z 1}\left(k_{x}^{2}+k_{y}^{2}\right)}\left[\begin{array}{ccc}
k_{y}^{2} & -k_{x} k_{y} & 0 \\
-k_{x} k_{y} & k_{x}^{2} & 0 \\
0 & 0 & 0
\end{array}\right], \\
\mathbf{M}^{\mathrm{p}}\left(\mathbf{k}_{\|}, z\right)=\frac{\mathrm{e}^{\xi j k_{11} z}}{k_{1}^{2}\left(k_{x}^{2}+k_{y}^{2}\right)}\left[\begin{array}{ccc}
k_{x}^{2} k_{z 1} & k_{x} k_{y} k_{z 1} & -\xi k_{x}\left(k_{x}^{2}+k_{y}^{2}\right) \\
k_{x} k_{y} k_{z 1} & k_{y}^{2} k_{z 1} & -\xi k_{y}\left(k_{x}^{2}+k_{y}^{2}\right) \\
-\xi k_{x}\left(k_{x}^{2}+k_{y}^{2}\right) & -\xi k_{y}\left(k_{x}^{2}+k_{y}^{2}\right) & \frac{\left(k_{x}^{2}+k_{y}^{2}\right)^{2}}{k_{z 1}}
\end{array}\right] .
\end{gathered}
$$

In the notation adopted here, the tensors $\mathbf{M}^{\mathrm{s}}$ and $\mathbf{M}^{\mathrm{p}}$ are the outer product of the vectors $\mathbf{L}_{\chi}^{\mathbf{n}}$ and $\mathbf{P}_{\chi}^{\mathbf{n}}$. The first vector is related to the amplitude of $\mathbf{E}_{\mathbf{i}}^{\mathbf{F T}}$, and each vector element corresponds to a dipole polarization, the index $n$ refers to the medium, and $\chi$ to the polarization $\left(\chi=s\right.$ for TE or $p$ for TM). The vector $\mathbf{P}_{\chi}^{\mathbf{n}}$ is responsible for decomposing the electric field in $x-, y$ - and $z$-components. Thus, $\mathbf{M}^{\mathrm{s}}$ and $\mathbf{M}^{\mathrm{p}}$ can be expressed as:

$$
\begin{aligned}
\mathbf{M}^{\mathbf{s}}\left(\mathbf{k}_{\|}, z\right)=\mathbf{P}_{\mathbf{s}}^{\mathbf{n}}\left(\mathbf{k}_{\|}\right) \otimes \mathbf{L}_{\mathbf{s}}^{\mathbf{n}}\left(\mathbf{k}_{\|}, z\right)=\left[\begin{array}{c}
\frac{k_{y}}{\sqrt{k_{x}^{2}+k_{y}^{2}}} \\
-\frac{k_{x}}{\sqrt{k_{x}^{2}+k_{y}^{2}}} \\
0
\end{array}\right] \otimes\left[\begin{array}{c}
\frac{k_{y}}{k_{z n} \sqrt{k_{x}^{2}+k_{y}^{2}}} e^{\xi j \mathbf{k}_{z n} z} \\
-\frac{k_{x}}{k_{z n} \sqrt{k_{x}^{2}+k_{y}^{2}}} e^{\xi \mathrm{j} \mathrm{k}_{z n} z} \\
0
\end{array}\right], \\
\mathbf{M}^{\mathbf{p}}\left(\mathbf{k}_{\|}, z\right)=\mathbf{P}_{\mathbf{p}}^{\mathbf{n}}\left(\mathbf{k}_{\|}\right) \otimes \mathbf{L}_{\mathbf{p}}^{\mathbf{n}}\left(\mathbf{k}_{\| \prime}, z\right)=\left[\begin{array}{c}
\frac{k_{x} k_{z n}}{k_{n} \sqrt{k_{x}^{2}+k_{y}^{2}}} \\
\frac{k_{y} k_{z n}}{k_{n} \sqrt{k_{x}^{2}+k_{y}^{2}}} \\
-\xi \frac{\sqrt{k_{x}^{2}+k_{y}^{2}}}{k_{n}}
\end{array}\right] \otimes\left[\begin{array}{l}
\frac{k_{x}}{k_{n} \sqrt{k_{x}^{2}+k_{y}^{2}}} e^{\xi j \mathbf{k}_{z n} z} \\
\frac{k_{y}}{k_{n} \sqrt{k_{x}^{2}+k_{y}^{2}}} e^{\xi j \mathbf{k}_{z n} z} \\
-\xi \frac{\sqrt{k_{x}^{2}+k_{y}^{2}}}{k_{n} k_{z n}} e^{ \pm j \mathbf{k}_{z n} z}
\end{array}\right] .
\end{aligned}
$$

The calculation of the dipole's dissipated power requires the knowledge of the total electric field present at the dipole location, which implies that the reflected electric field $\mathbf{E}_{\mathbf{r}}(\mathbf{r})$ needs also to be calculated. According to (4.4), the dipole's emission is an infinite sum of plane waves. Thus, we first consider a single plane wave with wavevector $k_{1}^{0}=$ $k_{x}^{0} \mathbf{x}+k_{y}^{0} \mathbf{y}+k_{z 1}^{0} \mathbf{z}$ being radiated by the dipole and impinging on the interface between 
medium 1 and $\operatorname{HMM}(z=q)$. The reflected field FT at the interface $\left(\mathbf{E}_{\mathbf{r}}^{\mathrm{FT}, \mathbf{0}}\left(\mathbf{k}_{\|}, \mathbf{k}_{\|}^{\mathbf{0}}, z=q\right)\right.$ is given by,

$$
\begin{aligned}
\mathbf{E}_{\mathbf{r}}^{\mathrm{FT}, 0}\left(\mathbf{k}_{\|}, \mathbf{k}_{\|}^{0}, q\right)= & \mathrm{j} \frac{\omega^{2} \mu_{1}}{4 \pi} \sum_{i, m=-\infty}^{\infty}\left\{\mathbf{P}_{\mathbf{s}}^{\mathbf{1}}\left(\mathbf{k}_{\|}\right) \otimes\left[\mathbf{L}_{\mathbf{s}}^{\mathbf{1}}\left(\mathbf{k}_{\|}^{0}, q\right) r_{s, s}^{i, m}\left(\mathbf{k}_{\|}^{0}\right)+\mathbf{L}_{\mathbf{p}}^{\mathbf{1}}\left(\mathbf{k}_{\|}^{0}, q\right) r_{p, s}^{i, m}\left(\mathbf{k}_{\|}^{0}\right)\right]\right. \\
& \left.+\mathbf{P}_{\mathbf{p}}^{\mathbf{1}}\left(\mathbf{k}_{\|}\right) \otimes\left[\mathbf{L}_{\mathbf{s}}^{\mathbf{1}}\left(\mathbf{k}_{\|}^{0}, q\right) r_{s, p}^{i, m}\left(\mathbf{k}_{\|}^{0}\right)+\mathbf{L}_{\mathbf{p}}^{\mathbf{1}}\left(\mathbf{k}_{\|}^{0}, q\right) r_{p, p}^{i, m}\left(\mathbf{k}_{\|}^{0}\right)\right]\right\} \\
& \cdot \mathbf{p} \delta\left(k_{x}-k_{x}^{0}-i K_{x}\right) \delta\left(k_{y}-k_{y}^{0}-m K_{y}\right)
\end{aligned}
$$

where $K_{x}=2 \pi / \Lambda_{x}$ and $K_{y}=2 \pi / \Lambda_{y}$ are the grating momenta along $x$ - and $y$-directions and $r_{\chi, \sigma}^{i, m}\left(\mathbf{k}_{\|}^{\mathbf{0}}\right)$ is the reflection coefficient of both the $i$ th diffraction order in $x$-direction and the $m$ th diffraction order in $y$-direction for the parallel incident wavevector $\mathbf{k}_{\|}^{\mathbf{0}} \cdot \chi$ and $\sigma$ indicate the polarization of the incident and reflected waves, respectively ( $\chi$ and $\sigma$ are equal to $p$ for TM polarization and $s$ for TE polarization). The reflection coefficient is calculated using the semi-analytical Rigorous Coupled Wave Analysis (RCWA) [152][154] method. For 3D gratings, cross polarization might occur, thus $r_{\chi, \sigma}^{i, m} \neq 0$ for $\chi \neq \sigma$. The next step is to sum all plane waves emitted by the dipole, which is done by integrating $\mathbf{E}_{\mathbf{r}}^{\mathrm{FT}, \mathbf{0}}$ over all possible $\mathbf{k}_{\|}^{\mathbf{0}}$. Thus, the FT of the reflected electric field emitted by the dipole at the interface $\mathbf{E}_{\mathbf{r}}^{\mathbf{F T}}$ is calculated as follows

$$
\begin{gathered}
\mathbf{E}_{\mathbf{r}}^{\mathrm{FT}}\left(\mathbf{k}_{\|}, q\right)=\iint \mathbf{E}_{\mathbf{r}}^{\mathrm{FT}, 0}\left(\mathbf{k}_{\|}, \mathbf{k}_{\|}^{0}, q\right) \mathrm{d} \mathbf{k}_{\|}^{0} \\
=\mathbf{j} \frac{\omega^{2} \mu_{1}}{4 \pi} \sum_{i, m=-\infty}^{\infty}\left\{\mathbf{P}_{\mathbf{s}}^{1}\left(\mathbf{k}_{\|}\right) \otimes\left[\mathbf{L}_{\mathbf{s}}^{1}\left(\mathbf{k}_{\|}^{0}, q\right) r_{s, s}^{i, m}\left(\mathbf{k}_{\|}^{0}\right)+\mathbf{L}_{\mathbf{p}}^{\mathbf{1}}\left(\mathbf{k}_{\|}^{0}, q\right) r_{p, s}^{i, m}\left(\mathbf{k}_{\|}^{0}\right)\right]\right. \\
\left.+\mathbf{P}_{\mathbf{p}}^{\mathbf{1}}\left(\mathbf{k}_{\|}\right) \otimes\left[\mathbf{L}_{\mathbf{s}}^{1}\left(\mathbf{k}_{\|}^{0}, q\right) r_{s, p}^{i, m}\left(\mathbf{k}_{\|}^{0}\right)+\mathbf{L}_{\mathbf{p}}^{\mathbf{1}}\left(\mathbf{k}_{\|}^{0}, q\right) r_{p, p}^{i, m}\left(\mathbf{k}_{\|}^{0}\right)\right]\right\} \mathbf{p}, \\
k_{x}^{0}=k_{x}-i K_{x}, \\
k_{y}^{0}=k_{y}-m K_{y} .
\end{gathered}
$$

Similarly, the calculation of the dipole's radiated power to the far field requires calculation of the transmitted electric field $\mathbf{E}_{t}(\mathbf{r})$. Using the same approach for $\mathbf{E}_{\mathbf{r}}^{\mathbf{F T}}$, the FT of $\mathbf{E}_{\mathbf{t}}$ at the interface between the grating upper surface and medium $2\left(\mathbf{E}_{\mathbf{t}}^{\mathbf{F T}}\left(\mathbf{k}_{\|}, z=z_{g r}\right)\right.$ ) is given by

$$
\begin{gathered}
\mathbf{E}_{\mathbf{t}}^{\mathrm{FT}}\left(\mathbf{k}_{\|}, z_{g r}\right)=\mathrm{j} \frac{\omega^{2} \mu_{1}}{4 \pi} \sum_{i, m=-\infty}^{\infty}\left\{\mathbf{P}_{\mathbf{s}}^{2}\left(\mathbf{k}_{\|}\right) \otimes\left[\mathbf{L}_{\mathbf{s}}^{1}\left(\mathbf{k}_{\|}^{0}, q\right) t_{s, s}^{i, m}\left(\mathbf{k}_{\|}^{0}\right)+\mathbf{L}_{\mathbf{p}}^{1}\left(\mathbf{k}_{\|}^{0}, q\right) t_{p, s}^{i, m}\left(\mathbf{k}_{\|}^{0}\right)\right]\right. \\
\left.+\mathbf{P}_{\mathbf{p}}^{2}\left(\mathbf{k}_{\|}\right) \otimes\left[\mathbf{L}_{\mathbf{s}}^{1}\left(\mathbf{k}_{\|}^{0}, q\right) t_{s, p}^{i, m}\left(\mathbf{k}_{\|}^{0}\right)+\mathbf{L}_{\mathbf{p}}^{\mathbf{1}}\left(\mathbf{k}_{\|}^{0}, q\right) t_{p, p}^{i, m}\left(\mathbf{k}_{\|}^{0}\right)\right]\right\} \mathbf{p} \\
k_{x}^{0}=k_{x}-i K_{x}, \\
k_{y}^{0}=k_{y}-m K_{y} .
\end{gathered}
$$


where $t_{\chi, \sigma}^{i, m}\left(\mathbf{k}_{\|}^{\mathbf{0}}\right)$ is the reflection coefficient of the $i$ th and $m$ th diffraction orders, respective to $x$ - and $y$-directions, for the incident wavevector $\mathbf{k}_{\mathbf{1}}^{\mathbf{0}}$ with $\chi$ and $\sigma$ as the polarization of the incident and the transmitted fields, respectively. The transmission coefficient is calculated using RCWA. The superscript 2 in $\mathbf{P}_{\mathbf{s}}^{2}\left(\mathbf{k}_{\|}\right)$and $\mathbf{P}_{\mathbf{p}}^{2}\left(\mathbf{k}_{\|}\right)$indicates the electric field is being decomposed in medium 2 . The reflected and transmitted electric fields are calculated by inverse Fourier transforming (IFT) their original fields, as follows,

$$
\begin{aligned}
\mathbf{E}_{\mathbf{r}}(\mathbf{r}) & =\frac{1}{2 \pi} \int_{-\infty}^{\infty} \int \mathbf{E}_{\mathbf{r}}^{\mathrm{FT}}\left(\mathbf{k}_{\|}, z\right) \mathrm{e}^{\mathrm{j}\left(k_{x} x+k_{y} y\right)} \mathrm{d} k_{x} \mathrm{~d} k_{y} \\
& =\frac{1}{2 \pi} \int_{-\infty}^{\infty} \int\left[\mathbf{E}_{\mathbf{r}}^{\mathrm{FT}}\left(\mathbf{k}_{\| \prime}, q\right) \mathrm{e}^{-j k_{z 1}(z-q)}\right] \mathrm{e}^{\mathrm{j}\left(k_{x} x+k_{y} y\right)} \mathrm{d} k_{x} \mathrm{~d} k_{y^{\prime}} \\
\mathbf{E}_{\mathbf{t}}(\mathbf{r}) & =\frac{1}{2 \pi} \int_{-\infty}^{\infty} \int \mathbf{E}_{\mathbf{t}}^{\mathrm{FT}}\left(\mathbf{k}_{\| \prime}, z\right) \mathrm{e}^{\mathrm{j}\left(k_{x} x+k_{y} y\right)} \mathrm{d} k_{x} \mathrm{~d} k_{y} \\
= & \frac{1}{2 \pi} \int_{-\infty}^{\infty} \int\left[\mathbf{E}_{\mathbf{t}}^{\mathrm{FT}}\left(\mathbf{k}_{\|}, z_{g r}\right) \mathrm{e}^{\mathrm{j} k_{z 1}\left(z-z_{g r}\right)}\right] \mathrm{e}^{\mathrm{j}\left(k_{x} x+k_{y} y\right)} \mathrm{d} k_{x} \mathrm{~d} k_{y^{\prime}}
\end{aligned}
$$

The solution proposed in this thesis assumes linear media, therefore the total electric field emitted by the dipole is the sum of the electric fields generated by each component of $\mathbf{p}$. The solutions are separated in two cases. The first case considers the dipole polarized along the $z$-axis, i.e., perpendicular to the HMM surface, while the second considers the dipole polarized along the $x$ - or $y$-axis, i.e., parallel to the HMM surface.

\subsubsection{Perpendicularly polarized dipole}

The polarization vector in this case has a component in $z$-direction, thus $\mathbf{p}=p_{z} \mathbf{z}$. Substituting $\mathbf{p}$ into (4.14) and (4.17), the FT of the reflected and transmitted electric fields become:

$$
\begin{gathered}
\mathbf{E}_{\mathbf{r}}^{\mathrm{FT}}\left(\mathbf{k}_{\|}, z\right)=\mathrm{j} \frac{\omega^{2} \mu}{4 \pi} p_{z}\left[R_{p, s}^{z}\left(\mathbf{k}_{\|}, q\right) \mathbf{P}_{s}^{1}\left(\mathbf{k}_{\|}\right)+R_{p, p}^{z}\left(\mathbf{k}_{\|}, q\right) \mathbf{P}_{\mathbf{p}}^{1}\left(\mathbf{k}_{\|}\right)\right] \mathrm{e}^{-\mathrm{j} k_{z 1}(z-q)}, \\
\mathbf{E}_{\mathbf{t}}^{\mathrm{FT}}\left(\mathbf{k}_{\|,}, z\right)=\mathrm{j} \frac{\omega^{2} \mu}{4 \pi} p_{z}\left[T_{p, s}^{z}\left(\mathbf{k}_{\| \prime}, z_{g r}\right) \mathbf{P}_{s}^{1}\left(\mathbf{k}_{\|}\right)+T_{p, p}^{z}\left(\mathbf{k}_{\| \prime}, z_{g r}\right) \mathbf{P}_{\mathbf{p}}^{1}\left(\mathbf{k}_{\|}\right)\right] \mathrm{e}^{\mathrm{j} k_{z 1}\left(z-z_{g r}\right)}, \\
R_{p, \sigma}^{z}\left(\mathbf{k}_{\|,}, q\right)=-\sum_{i, m=-\infty}^{\infty} \frac{\sqrt{\left(k_{x}^{0}\right)^{2}+\left(k_{y}^{0}\right)^{2}}}{k_{z 1}^{0} k_{1}^{j}} e^{k_{z 1}^{0} q} r_{p, \sigma}^{i, m}\left(\mathbf{k}_{\|}^{0}\right), \\
T_{p, \sigma}^{z}\left(\mathbf{k}_{\|}, z_{g^{r}}\right)=-\sum_{i, m=-\infty}^{\infty} \frac{\sqrt{\left(k_{x}^{0}\right)^{2}+\left(k_{y}^{0}\right)^{2}}}{k_{z 1}^{0} k_{1}} \mathrm{e}^{\mathrm{j} k_{z 1}^{0} q} t_{p, \sigma}^{i, m}\left(\mathbf{k}_{\|}^{0}\right) .
\end{gathered}
$$

With the reflected and transmitted electric fields calculated, the next step is to obtain the Purcell factor and the dipole's total radiated power into medium 2, as described next. The first step is to determine the dipole's total dissipated power $(W)$, which is carried out as follows: 


$$
\begin{gathered}
W=\frac{\omega}{2} \operatorname{Im}\left\{\mathbf{p}^{*} \cdot \mathbf{E}_{\mathbf{i}}\left(\mathbf{r}_{0}\right)+\mathbf{p}^{*} \cdot \mathbf{E}_{\mathbf{r}}\left(\mathbf{r}_{0}\right)\right\}, \\
W=\frac{\omega}{2} \operatorname{Im}\left\{\frac{1}{2 \pi} \int_{-\infty}^{\infty} \int\left[\mathrm{j} \frac{\omega^{2} \mu_{1}}{4 \pi} p_{z}^{2} \frac{k_{x}^{2}+k_{y}^{2}}{k_{1}^{2} k_{z 1}}+\mathrm{j} \frac{\omega^{2} \mu_{1}}{4 \pi} p_{z}^{2}\left(\begin{array}{l}
R_{p, s}^{z}\left(\mathbf{k}_{\|}, q\right) \mathbf{P}_{s}^{1}\left(\mathbf{k}_{\|}\right)+ \\
R_{p, p}^{z}\left(\mathbf{k}_{\|}, q\right) \mathbf{P}_{\mathbf{p}}^{1}\left(\mathbf{k}_{\|}\right)
\end{array}\right) \cdot \mathbf{z} \mathrm{e}^{\mathrm{j} k_{z 1} q}\right] \mathrm{d} k_{x} \mathrm{~d} k_{y}\right\} \\
=\frac{\omega^{3} \mu_{1}}{16 \pi^{2}} p_{z}^{2} \operatorname{Re}\left\{\int_{-\infty}^{\infty} \int\left[\frac{k_{x}^{2}+k_{y}^{2}}{k_{1}^{2} k_{z 1}}+\left(R_{p, s}^{z}\left(\mathbf{k}_{\|}, q\right) \mathbf{P}_{s}^{1}\left(\mathbf{k}_{\|}\right)+R_{p, p}^{z}\left(\mathbf{k}_{\|}, q\right) \mathbf{P}_{\mathbf{p}}^{1}\left(\mathbf{k}_{\|}\right)\right) \cdot \mathbf{z} e^{j k_{21} q}\right] \mathrm{d} k_{x} \mathrm{~d} k_{y}\right\} .
\end{gathered}
$$

The Purcell factor is defined as the ratio of the dipole's dissipated power to that it would dissipate in free space $\left(W_{0}=p_{z}^{2} \omega k_{0}^{3} / 12 \pi \epsilon_{0}\right)$. Since $\mathbf{P}_{\mathbf{s}}^{\mathbf{1}}\left(\mathbf{k}_{\|}\right) \cdot \mathbf{z}=0$, the Purcell factor for perpendicular polarization $\left(P_{z}\right)$ is given by:

$$
P_{z}=\frac{W}{W_{0}}=\frac{k_{1}}{k_{0}}+\frac{3}{4 \pi k_{0}} \operatorname{Re}\left\{\int_{-\infty}^{\infty} \int\left[R_{p, p}^{z}\left(\mathbf{k}_{\|}, q\right) \mathbf{P}_{\mathbf{p}}^{1}\left(\mathbf{k}_{\|}\right)\right] \cdot \mathbf{z} e^{j k_{z 1} q} \mathrm{~d} k_{x} \mathrm{~d} k_{y}\right\} .
$$

Our main goal is to be able to increase the coupling of evanescent waves inside the HMM to propagating waves in the medium 2 . In this sense, it is important to calculate the dipole's total emitted power into the far field in medium 2. Using the stationary phase method [109], the transmitted electric field at the far field $\left(\mathbf{E}_{\mathbf{z}}^{\mathbf{F F}}\right)$ is calculated as follows,

$$
\begin{aligned}
& \mathbf{E}_{z}^{\mathrm{FF}}(\mathbf{r})=-\mathbf{j} k_{z 2} \mathbf{E}_{\mathbf{t}}^{\mathrm{FT}}\left(\mathbf{k}_{\|}, 0\right) \frac{e^{j \mid \mathbf{k}_{2} / r}}{r} \\
& =\frac{\omega^{2} \mu_{1}\left|\mathbf{k}_{2}\right|}{4 \pi} p_{z} \cos \theta\left[T_{p, s}^{z}\left(\mathbf{k}_{\| \mid}, z_{g r}\right) \boldsymbol{\varphi}+T_{p, p}^{z}\left(\mathbf{k}_{\| \mid}, z_{g r}\right) \boldsymbol{\theta}\right] \frac{e^{j\left|\mathbf{k}_{2}\right| r}}{r} e^{-j\left|\mathbf{k}_{2}\right| \cos \theta z_{g r}},
\end{aligned}
$$

where $r, \theta$ and $\varphi$ are the components of $\mathbf{r}$ in spherical coordinates. For the far field equation to be used in (4.29) the following transformation is required:

$$
\mathbf{k}_{2}=\left[\begin{array}{c}
k_{x} \\
k_{y} \\
k_{z 2}
\end{array}\right]=k_{2}\left[\begin{array}{c}
\sin \theta \cos \varphi \\
\sin \theta \sin \varphi \\
\cos \theta
\end{array}\right] \begin{aligned}
& 0 \leq \theta \leq \pi / 2 \\
& 0 \leq \varphi \leq 2 \pi
\end{aligned}
$$

The transformation of $\mathbf{E}_{\mathbf{z}}^{\mathbf{F F}}(\mathbf{r})$ into spherical coordinates is carried out after substituting (4.29) into (4.11) and (4.12), which gives

$$
\begin{gathered}
\mathbf{P}_{\mathbf{p}}^{2}\left(\mathbf{k}_{\|}\right)=\frac{k_{x} k_{z 2}}{k_{2} \sqrt{k_{x}^{2}+k_{y}^{2}}} \mathbf{x}+\frac{k_{y} k_{z 2}}{k_{2} \sqrt{k_{x}^{2}+k_{y}^{2}}} \mathbf{y}-\frac{\sqrt{k_{x}^{2}+k_{y}^{2}}}{k_{2}} \mathbf{z}=\boldsymbol{\theta}, \\
\mathbf{P}_{\mathbf{s}}^{2}\left(\mathbf{k}_{\|}\right)=\frac{k_{y}}{\sqrt{k_{x}^{2}+k_{y}^{2}}} \mathbf{x}-\frac{k_{x}}{\sqrt{k_{x}^{2}+k_{y}^{2}}} \mathbf{y}=\boldsymbol{\varphi} .
\end{gathered}
$$

With $\mathbf{E}_{\mathbf{z}}^{\mathbf{F F}}$ known, the time-averaged Poynting vector $\left(\left\langle\boldsymbol{S}_{\mathbf{z}}(\mathbf{r})\right\rangle\right)$ is calculated as follows, 


$$
\begin{aligned}
\left\langle\mathbf{S}_{z}(\mathbf{r})\right\rangle & =\frac{1}{2 z_{2}} \operatorname{Re}\left\{\mathbf{E}_{z}^{\mathrm{FF}} \cdot \mathbf{E}_{z}^{\mathrm{FF}}\right\} \mathbf{r} \\
& =\frac{\omega^{4} \mu_{1}^{2} k_{2}}{32 \pi^{2} z_{2} r^{2}} p_{z}^{2} \cos ^{2} \theta\left(\left|T_{p, s}^{z}\left(\mathbf{k}_{\|}, z_{g r}\right)\right|^{2}+\left|T_{p, p}^{z}\left(\mathbf{k}_{\|}, z_{g r}\right)\right|^{2}\right) \mathbf{r} .
\end{aligned}
$$

The total radiated power into the far field $\left(Q_{z}\right)$ of a perpendicularly polarized dipole is calculated integrating $\left\langle\mathbf{S}_{\mathbf{z}}\right\rangle$ over the upper semi-sphere, i.e.

$$
\begin{aligned}
Q_{z} & =\int_{0}^{\pi / 2} \int_{0}^{2 \pi}\left\langle\mathbf{S}_{\mathbf{z}}\right\rangle \cdot \mathbf{r} \sin \theta r^{2} \mathrm{~d} \varphi \mathrm{d} \theta \\
& =\frac{\omega^{4} \mu_{1}^{2} k_{2} p_{z}^{2}}{32 \pi^{2} z_{2}} \int_{0}^{\pi / 2} \int_{0}^{2 \pi} \cos ^{2} \theta \sin \theta\left(\left|T_{p, s}^{z}\left(\mathbf{k}_{\|}, z_{g r}\right)\right|^{2}+\left|T_{p, p}^{z}\left(\mathbf{k}_{\|}, z_{g r}\right)\right|^{2}\right) \mathrm{d} \varphi \mathrm{d} \theta .
\end{aligned}
$$

\subsubsection{Parallel polarized dipole}

The polarization vector in this case is oriented along the $x$-axis, resulting in $\mathbf{p}=p_{x} \mathbf{x}$. The equations derived in this section can be easily adapted to the $y$-axis by simply changing the terms $k_{x}$ to $k_{y}$, and $k_{y}$ to $k_{x}$. After substituting $\mathbf{p}$ into (4.14) and (4.17), the following expressions for the FTs of the reflected and transmitted electric fields are obtained:

$$
\begin{aligned}
& \mathbf{E}_{\mathbf{r}}^{\mathrm{FT}}\left(\mathbf{k}_{\|}, z\right)=\mathrm{j} \frac{\omega^{2} \mu}{4 \pi} p_{x}\left\{\left[R_{s, s}^{x}\left(\mathbf{k}_{\|}, q\right)+R_{p, s}^{x}\left(\mathbf{k}_{\|}, q\right)\right] \mathbf{P}_{s}^{1}\left(\mathbf{k}_{\|}\right)\right. \\
& \left.+\left[R_{p, p}^{x}\left(\mathbf{k}_{\|}, q\right)+R_{s, p}^{x}\left(\mathbf{k}_{\|}, q\right)\right] \mathbf{P}_{\mathbf{p}}^{1}\left(\mathbf{k}_{\|}\right)\right\} \mathrm{e}^{-\mathrm{j} k_{z 1}(z-q)}, \\
& \mathbf{E}_{\mathbf{t}}^{\mathrm{FT}}\left(\mathbf{k}_{\|}, z\right)=\mathrm{j} \frac{\omega^{2} \mu}{4 \pi} p_{x}\left\{\left[T_{s, s}^{x}\left(\mathbf{k}_{\|}, z_{g r}\right)+T_{p, s}^{x}\left(\mathbf{k}_{\|}, z_{g^{r}}\right)\right] \mathbf{P}_{\mathrm{s}}^{2}\left(\mathbf{k}_{\|}\right)\right. \\
& \left.+\left[T_{p, p}^{x}\left(\mathbf{k}_{\|}, z_{g r}\right)+T_{s, p}^{x}\left(\mathbf{k}_{\|}, z_{g r}\right)\right] \mathbf{P}_{\mathbf{p}}^{2}\left(\mathbf{k}_{\|}\right)\right\} e^{j k_{z 1}\left(z-z_{g r}\right)}, \\
& R_{s, \sigma}^{x}\left(\mathbf{k}_{\|,}, q\right)=\sum_{i, m=-\infty}^{\infty} \frac{k_{y}^{0}}{k_{z 1}^{0} \sqrt{\left(k_{x}^{0}\right)^{2}+\left(k_{y}^{0}\right)^{2}}} e^{j k_{z 1}^{0} q} r_{s, \sigma}^{i, m}\left(\mathbf{k}_{\|}^{0}\right),
\end{aligned}
$$

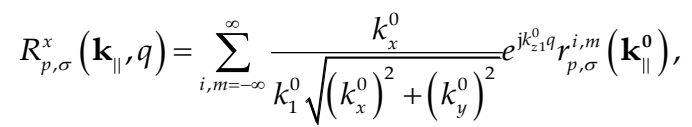

$$
\begin{aligned}
& T_{s, \sigma}^{x}\left(\mathbf{k}_{\|}, z_{g r}\right)=\sum_{i, m=-\infty}^{\infty} \frac{k_{y}^{0}}{k_{z 1}^{0} \sqrt{\left(k_{x}^{0}\right)^{2}+\left(k_{y}^{0}\right)^{2}}} e^{j k_{z 1}^{0} q} t_{s, \sigma}^{i, m}\left(\mathbf{k}_{\|}^{0}\right), \\
& T_{p, \sigma}^{x}\left(\mathbf{k}_{\|}, z_{g r}\right)=\sum_{i, m=-\infty}^{\infty} \frac{k_{x}^{0}}{k_{1}^{0} \sqrt{\left(k_{x}^{0}\right)^{2}+\left(k_{y}^{0}\right)^{2}}} e^{j k_{z 1}^{0} q} t_{p, \sigma}^{i, m}\left(\mathbf{k}_{\|}^{0}\right) .
\end{aligned}
$$

The Purcell factor for parallel polarization is obtained after substituting (4.35) into (4.26) and the resulting equation into (4.28), which gives: 


$$
\begin{aligned}
P_{x} & =\frac{k_{1}}{k_{0}}+\frac{3}{4 \pi k_{0}} \operatorname{Re}\left\{\int _ { - \infty } ^ { \infty } \int \left[\left(R_{s, s}^{x}\left(\mathbf{k}_{\|}, q\right)+R_{p, s}^{x}\left(\mathbf{k}_{\|}, q\right)\right) \mathbf{P}_{s}^{1}\left(\mathbf{k}_{\|}\right)+\right.\right. \\
& \left.\left.+\left(R_{p, p}^{x}\left(\mathbf{k}_{\|,}, q\right) R_{s, p}^{x}\left(\mathbf{k}_{\|}, q\right)\right) \mathbf{P}_{\mathbf{p}}^{1}\left(\mathbf{k}_{\|}\right)\right] \cdot \mathbf{x} e^{j k_{z 1} q} \mathrm{~d} k_{x} \mathrm{~d} k_{y}\right\}
\end{aligned}
$$

The total dipole's radiated power into the far field requires the electric field calculation in the far field $\left(\mathbf{E}_{\mathbf{x}}^{\mathbf{F F}}\right)$. As in the previous section, this is accomplished with the stationary phase method [109] as follows,

$$
\begin{aligned}
\mathbf{E}_{x}^{\mathrm{FF}}(\mathbf{r}) & =-\mathbf{j} k_{z 2} \mathbf{E}_{\mathbf{t}}^{\mathrm{FT}}\left(\mathbf{k}_{\|}, 0\right) \frac{e^{\mathrm{j} k_{2} r}}{r} \\
& =\frac{\omega^{2} \mu_{1} k_{2}}{4 \pi} p_{x} \cos \theta\left\{\left[T_{s, s}^{x}\left(\mathbf{k}_{\|}, z_{g r}\right)+T_{p, s}^{x}\left(\mathbf{k}_{\| \prime}, z_{g r}\right)\right] \boldsymbol{\varphi}\right. \\
& \left.+\left[T_{s, p}^{x}\left(\mathbf{k}_{\|}, z_{g r}\right)+T_{p, p}^{x}\left(\mathbf{k}_{\|}, z_{g r}\right)\right] \boldsymbol{\theta}\right\} \frac{e^{\mathrm{j} k_{2} r}}{r} e^{-\mathrm{j} k_{2} \cos \theta z_{g r}}
\end{aligned}
$$

The far field approximation in (4.42) also makes use of (4.30). Equations (4.31) and (4.32) transform $\mathbf{E}_{\mathbf{x}}^{\mathbf{F F}}$ into spherical coordinates, from which the time-averaged Poynting vector $\left(\left\langle\mathbf{S}_{\mathbf{x}}\right\rangle\right)$ is calculated as follows,

$$
\begin{aligned}
& \left\langle\mathbf{S}_{\mathbf{x}}(\mathbf{r})\right\rangle=\frac{1}{2 z_{2}} \operatorname{Re}\left\{\mathbf{E}_{\mathbf{t}}^{\mathrm{FF}} \cdot \mathbf{E}_{\mathbf{t}}^{\mathrm{FF}}\right\} \mathbf{r} \\
& =\frac{\omega^{4} \mu_{1}^{2} k_{2}}{32 \pi^{2} z_{2} r^{2}} p_{x}^{2} \cos ^{2} \theta\left(\left|T_{s, s}^{x}\left(\mathbf{k}_{\|}, z_{g r}\right)+T_{p, s}^{x}\left(\mathbf{k}_{\|}, z_{g r}\right)\right|^{2}+\left|T_{s, p}^{x}\left(\mathbf{k}_{\|}, z_{g r}\right)+T_{p, p}^{x}\left(\mathbf{k}_{\|}, z_{g r}\right)\right|^{2}\right) \mathbf{r} .
\end{aligned}
$$

The total radiated power into the far field $\left(Q_{x}\right)$ by a parallel polarized dipole is calculated using the same approach adopted for the perpendicularly polarized case, which gives,

$$
\begin{aligned}
Q_{x} & =\int_{0}^{\pi / 2} \int_{0}^{2 \pi}\left\langle\mathbf{S}_{\mathbf{x}}\right\rangle \cdot \mathbf{r} r^{2} \sin \theta \mathrm{d} \varphi \mathrm{d} \theta \\
& =\frac{\omega^{4} \mu_{1}^{2} k_{2} p_{x}^{2}}{32 \pi^{2} z_{2}} \int_{0}^{\pi / 2} \int_{0}^{2 \pi} \cos ^{2} \theta \sin \theta\left(\left|T_{s, s}^{x}\left(\mathbf{k}_{\| \prime}, z_{g^{r}}\right)+T_{p, s}^{x}\left(\mathbf{k}_{\|}, z_{g r}\right)\right|^{2}\right. \\
& \left.+\left|T_{s, p}^{x}\left(\mathbf{k}_{\|}, z_{g r}\right)+T_{p, p}^{x}\left(\mathbf{k}_{\| \prime}, z_{g r}\right)\right|^{2}\right) \mathrm{d} \varphi \mathrm{d} \theta .
\end{aligned}
$$

\subsubsection{D System}

For the $2 \mathrm{D}$ case we consider the dipole embedded in the medium 1 (with permittivity $\varepsilon 1$ ) at a distance $q$ from the HMM bottom surface, as shown in Figure 4.2. The HMM and the grating are merged in this figure for sake of clarity. The grating is surrounded by medium 2 (with permittivity $\varepsilon_{2}$ ) and has periodicity $\Lambda_{x}$ along the $x$-axis. The most significant difference between the $3 \mathrm{D}$ and $2 \mathrm{D}$ approaches is that the system is constant along the $y$ axis, i.e. $\partial / \partial y=0$, resulting in $k_{y}=0$. Consequently, an excitation point in the $x z$ plane, defined here as a $2 \mathrm{D}$ dipole, behaves as an excitation line along the $y$-axis. Therefore, the 


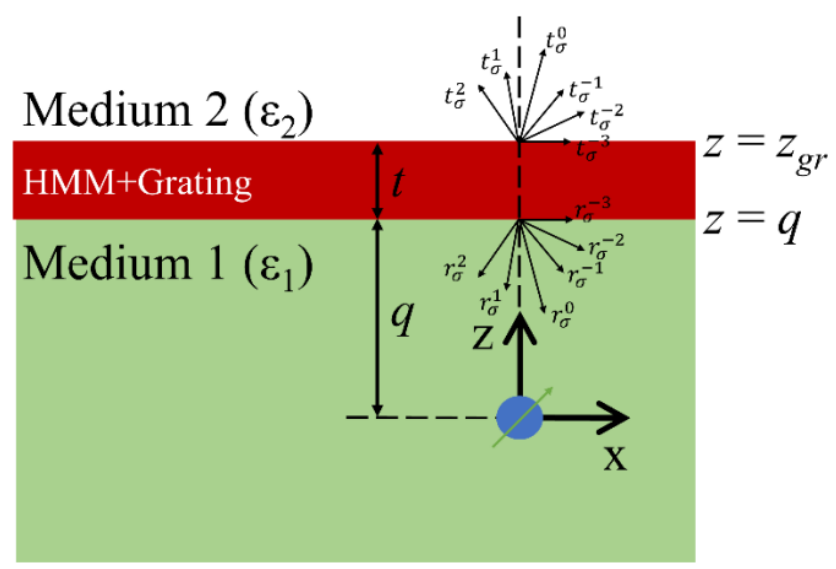

Figure 4.2. Proposed 2D geometry in which the QEs are modelled as dipoles embedded in medium 1 (with permittivity $\varepsilon 1$ ), centered at the origin and at a distance $\mathrm{q}$ from the HMM bottom surface. The grating with periodicity $\Lambda_{x}$ in $x$-direction is on top of the HMM and is covered with medium 2 (with permittivity $\varepsilon 2$ ). The HMM and the grating are merged for sake of clarity.

procedure to calculate the Purcell factor and the radiated power is different from the 3D scenario and starts with the calculation of the transmitted and reflected electric fields.

\subsubsection{Calculation of the transmitted and reflected electric fields}

We start by calculating the electric field radiated by the dipole in medium 1 , which requires the dipole to be modelled as the following current source in 2D,

$$
\mathbf{J}(\mathbf{r})=-\mathbf{j} \omega \delta^{(2)}(x, z) \mathbf{p}
$$

where $\mathbf{p}$ is the dipole momentum and $\delta^{(2)}(x, z)$ is the 2D Dirac delta function. The electric field radiated by the dipole $\left(\mathbf{E}_{\mathbf{i}}\right)$ in $2 \mathrm{D}$ becomes

$$
\begin{gathered}
\mathbf{E}_{\mathbf{i}}(\mathbf{Q})=\omega^{2} \mu_{1} \iint_{A^{\prime}}\left(\mathbf{I}+\frac{1}{k_{1}^{2}} \nabla \nabla\right) \mathrm{G}_{0}\left(\mathbf{Q}-\mathbf{Q}^{\prime}\right) \delta^{(2)}\left(x^{\prime}, z^{\prime}\right) \mathbf{p d} A^{\prime}, \\
\mathrm{G}_{0}(\mathbf{Q})=\frac{\mathrm{j}}{4} H_{0}^{1}\left(\mathbf{k}_{1} \mathbf{Q}\right)=\mathrm{j} \frac{1}{4 \pi} \int_{-\infty}^{\infty} \frac{1}{\sqrt{k_{0}^{2}-k_{x}^{2}}} \mathrm{e}^{\mathrm{j}\left(k_{x} x+\xi k_{z 1} z\right)} \mathrm{d} k_{x} \\
\mathbf{Q}=x \mathbf{X}+z \mathbf{Z},
\end{gathered}
$$

where $H_{0}^{1}$ is the first Hankel function of order 0, G0 is the scalar Green's function in 2D [155] and $\varrho$ is the position vector in 2D. Substituting (4.47) into (4.46), the following expression for $\mathbf{E}(\mathbf{Q})$ is obtained 


$$
\mathbf{E}_{\mathbf{i}}(\mathbf{\varrho})=\mathrm{j} \frac{\omega^{2} \mu_{1}}{4 \pi}\left(\mathbf{I}+\frac{1}{k_{1}^{2}} \nabla \nabla\right) \int_{-\infty}^{\infty} \frac{1}{k_{z 1}} \mathrm{e}^{\mathrm{j}\left(k_{x} x+\xi k_{z 1}\right)} \mathbf{p} \mathrm{d} k_{x} .
$$

By considering the 2D FT and the inverse FT defined as

$$
\begin{aligned}
& \mathbf{E}_{\mathbf{i}}^{\mathrm{FT}}\left(k_{x}, z\right)=\frac{1}{\sqrt{2 \pi}} \int_{-\infty}^{\infty} \mathbf{E}_{\mathbf{i}}(\mathbf{r}) \mathrm{e}^{-\mathrm{j} k_{x} x} \mathrm{~d} x, \\
& \mathbf{E}_{\mathbf{i}}(\mathbf{Q})=\frac{1}{\sqrt{2 \pi}} \int_{-\infty}^{\infty} \mathbf{E}_{\mathbf{i}}^{\mathrm{FT}}\left(k_{x}, z\right) \mathrm{e}^{\mathrm{j}\left(k_{x} x\right)} \mathrm{d} k_{x},
\end{aligned}
$$

we obtain

$$
\mathbf{E}_{\mathbf{i}}^{\mathrm{FT}}\left(k_{x}, z\right)=\mathrm{j} \frac{\omega^{2} \mu_{1}}{\sqrt{8 \pi}} \frac{1}{k_{1}^{2} k_{z 1}}\left[\begin{array}{ccc}
k_{1}^{2}-k_{x}^{2} & 0 & -\xi k_{x} k_{z 1} \\
0 & k_{1}^{2} & 0 \\
-\xi k_{x} k_{z 1} & 0 & k_{1}^{2}-k_{z 1}^{2}
\end{array}\right] \mathrm{e}^{\mathrm{j} \xi k_{z 1} z}=\mathrm{j} \frac{\omega^{2} \mu_{1}}{\sqrt{8 \pi}} \mathbf{M}\left(k_{x}, z\right) \mathbf{p} .
$$

In 2D, the dyadic tensor $\mathbf{M}$ has TE and TM components and can be written as the outer product of two vectors, $\mathbf{L}_{\chi}^{\mathbf{n}}$ and $\mathbf{P}_{\chi^{\prime}}^{\mathbf{n}}$, whose physical meaning is the same as for the 3D case. Thus, $\mathbf{M}$ is written as:

$$
\begin{gathered}
\mathbf{M}\left(\mathbf{k}_{\|}, z\right)=\mathbf{M}^{\mathrm{s}}\left(\mathbf{k}_{\| \mid}, z\right)+\mathbf{M}^{\mathbf{p}}\left(\mathbf{k}_{\| \prime}, z\right) \\
\mathbf{M}^{\mathrm{s}}\left(k_{x}, z\right)=\mathbf{P}_{\mathbf{s}}^{1}\left(k_{x}\right) \otimes \mathbf{L}_{\mathbf{s}}^{1}\left(k_{x}, z\right)=\left[\begin{array}{l}
0 \\
1 \\
0
\end{array}\right] \otimes\left[\begin{array}{c}
0 \\
\frac{1}{k_{z 1}} \mathrm{e}^{\xi j k_{z 1} z} \\
0
\end{array}\right] . \\
\mathbf{M}^{\mathrm{p}}\left(k_{x}, z\right)=\mathbf{P}_{\mathbf{p}}^{\mathbf{1}}\left(k_{x}\right) \otimes \mathbf{L}_{\mathbf{p}}^{1}\left(k_{x}, z\right)=\left[\begin{array}{c}
\frac{k_{z 1}}{k_{1}} \\
0 \\
-\xi \frac{k_{x}}{k_{1}}
\end{array}\right] \otimes\left[\begin{array}{c}
\frac{1}{k_{1}} \mathrm{e}^{\xi j k_{z 1} z} \\
0 \\
-\xi \frac{k_{x}}{k_{1} k_{z 1}} \mathrm{e}^{\xi j k_{z 1} z}
\end{array}\right] .
\end{gathered}
$$

The dipole only generates TE waves if polarized along y-axis. Nevertheless, if $\mathbf{p}$ is in the xz-plane, only TM waves are generated. Since we are working with hyperbolic metamaterials, which only couples TM waves, for the $2 \mathrm{D}$ case, we will consider $\mathbf{p}$ in $\mathrm{xz}$ plane. The calculation of the reflected electric field is based on the same approach used for the 3D case. Firstly, we consider a single plane wave with wavevector $\mathbf{k}_{\mathbf{1}}^{\mathbf{0}}=k_{x}^{0} \mathbf{x}+k_{z 1}^{0} \mathbf{z}$ impinging on the interface between medium 1 and $\operatorname{HMM}(z=q)$. The FT of the reflected field $\left(\mathbf{E}_{\mathbf{r}}^{\mathbf{F T}, \mathbf{0}}\left(k_{x}, k_{x}^{0}, q\right)\right)$ of this wave at $z=q$ is given by,

$$
\mathbf{E}_{\mathbf{r}}^{\mathrm{FT}, 0}\left(k_{x}, k_{x}^{0}, q\right)=\mathrm{j} \frac{\omega^{2} \mu_{1}}{\sqrt{8 \pi}} \sum_{i=-\infty}^{\infty}\left\{\mathbf{P}_{\mathbf{p}}^{\mathbf{1}}\left(k_{x}\right) \otimes\left[\mathbf{L}_{\mathbf{p}}^{\mathbf{1}}\left(k_{x}^{0}, q\right) r_{p}^{i}\left(k_{x}^{0}\right)\right]\right\} \mathbf{p} \delta\left(k_{x}-k_{x}^{0}-i K_{x}\right),
$$


where $K_{x}=2 \pi / \Lambda_{x}$ is the grating momentum in $x$-direction and $r_{p}^{i}$ is the reflection coefficient of the $i$ th diffraction order of a $p$-polarized wave. In $2 \mathrm{D}$, all dipoles radiate $p$ polarized waves, therefore the magnetic field is oriented along the $y$-axis. Since the grating changes the wave momentum in the $x z$ plane, only the electric field vector, which is in the $x z$ plane, changes its direction. Therefore, the wave remains $p$-polarized and no cross-polarization coefficients appear in (4.52). Thus, the FT of the reflected electric field radiated by the dipole $\mathbf{E}_{\mathbf{r}}^{\mathbf{F T}}$ can be calculated integrating $\mathbf{E}_{\mathbf{r}}^{\mathrm{FT}, \mathbf{0}}$ over all possible $k_{x}^{0}$, i.e.,

$$
\begin{aligned}
& \mathbf{E}_{\mathbf{r}}^{\mathrm{FT}}\left(k_{x}, q\right)=\int_{-\infty}^{\infty} \mathbf{E}_{\mathbf{r}}^{\mathrm{FT}, 0}\left(k_{x}, k_{x}^{0}, q\right) \mathrm{d} k_{x}^{0} \\
&=\int_{-\infty}^{\infty} \mathrm{j} \frac{\omega^{2} \mu_{1}}{\sqrt{8 \pi}} \sum_{i}\left\{\mathbf{P}_{\mathbf{p}}^{\mathbf{1}}\left(k_{x}\right) \otimes\left[\mathbf{L}_{\mathbf{p}}^{\mathbf{1}}\left(k_{x}^{0}, q\right) r_{p}^{i}\left(k_{x}^{0}\right)\right]\right\} \mathbf{p} \delta\left(k_{x}-k_{x}^{0}-i K_{x}\right) \mathrm{d} k_{x}^{0} \\
&=\mathrm{j} \frac{\omega^{2} \mu_{1}}{\sqrt{8 \pi}} \sum_{i}\left\{\mathbf{P}_{\mathbf{p}}^{1}\left(k_{x}\right) \otimes\left[\mathbf{L}_{\mathbf{p}}^{1}\left(k_{x}^{0}, q\right) r_{p}^{i}\left(k_{x}^{0}\right)\right]\right\} \mathbf{p}, \\
& k_{x}^{0}=k_{x}-i K_{x} .
\end{aligned}
$$

The FT of the transmitted electric field $\left(\mathbf{E}_{\mathbf{t}}^{\mathbf{F T}}\right)$ follows the same methodology adopted for the reflected field. Thus, at the interface between the grating upper surface and medium $2\left(z=z_{g r}\right)$ we obtain,

$$
\mathbf{E}_{\mathbf{t}}^{\mathrm{FT}}\left(k_{x}, z_{g r}\right)=\mathrm{j} \frac{\omega^{2} \mu_{1}}{\sqrt{8 \pi}} \sum_{i}\left\{\mathbf{P}_{\mathbf{p}}^{2}\left(k_{x}\right) \otimes\left[\mathbf{L}_{\mathbf{p}}^{1}\left(k_{x}^{0}, q\right) t_{p}^{i}\left(k_{x}^{0}\right)\right]\right\} \mathbf{p}
$$

The superscript 2 in $\mathbf{P}_{\mathbf{p}}^{2}\left(k_{x}\right)$ indicates the electric field is being decomposed in medium 2 . Since we have the FT of the transmitted and reflected fields, the electric fields in the spatial domain are obtained using the IFT, as follows,

$$
\begin{aligned}
& \mathbf{E}_{\mathbf{r}}(\mathbf{Q})=\frac{1}{\sqrt{2 \pi}} \int_{-\infty}^{\infty} \mathbf{E}_{\mathbf{r}}^{\mathrm{FT}}\left(k_{x}, z\right) \mathrm{e}^{\mathrm{j} k_{x} x} \mathrm{~d} k_{x}=\frac{1}{\sqrt{2 \pi}} \int_{-\infty}^{\infty}\left[\mathbf{E}_{\mathbf{r}}^{\mathrm{FT}}\left(k_{x}, q\right) \mathrm{e}^{-j k_{z 1}(z-q)}\right] \mathrm{e}^{\mathrm{j} k_{x} x} \mathrm{~d} k_{x}, \\
& \mathbf{E}_{\mathbf{t}}(\mathbf{Q})=\frac{1}{\sqrt{2 \pi}} \int_{-\infty}^{\infty} \mathbf{E}_{\mathbf{t}}^{\mathrm{FT}}\left(k_{x}, z\right) \mathrm{e}^{\mathrm{j} k_{x} x} \mathrm{~d} k_{x}=\frac{1}{\sqrt{2 \pi}} \int_{-\infty}^{\infty}\left[\mathbf{E}_{\mathbf{t}}^{\mathrm{FT}}\left(k_{x}, q\right) \mathrm{e}^{\mathrm{j} k_{z 1}\left(z-z_{g r}\right)}\right] \mathrm{e}^{\mathrm{j} k_{x} x} \mathrm{~d} k_{x},
\end{aligned}
$$

In $2 \mathrm{D}, \mathbf{p}$ can be decomposed in $x$ and $z$ components, therefore the solutions hereon are separated into two cases. The first considers the dipole polarized along the $z$-axis, i.e., perpendicular to the HMM surface, while the second considers the dipole polarized along the $x$-axis, i.e., parallel to the HMM surface.

\subsubsection{Perpendicularly polarized dipole}

The dipole moment in this case is polarized along the $z$-axis, thus $\mathbf{p}=p_{z} \mathbf{z}$. After substituting $\mathbf{p}$ into (4.57) and (4.59), the following FTs of the reflected and transmitted field electric are obtained: 


$$
\begin{gathered}
\mathbf{E}_{\mathbf{r}}^{\mathbf{F T}}\left(k_{x}, z\right)=\mathrm{j} \frac{\omega^{2} \mu_{1}}{\sqrt{8 \pi}} p_{z} R_{p}^{z}\left(k_{x}, q\right) \mathbf{P}_{\mathbf{p}}^{1}\left(k_{x}\right) e^{-j k_{z 1}(z-q)}, \\
\mathbf{E}_{\mathbf{t}}^{\mathbf{F T}}\left(k_{x}, z\right)=\mathrm{j} \frac{\omega^{2} \mu_{1}}{\sqrt{8 \pi}} p_{z} T_{p}^{z}\left(k_{x}, z_{g r}\right) \mathbf{P}_{\mathbf{p}}^{2}\left(k_{x}\right) e^{j k_{z 1}\left(z-z_{g r}\right)}, \\
R_{p}^{z}\left(k_{x}, q\right)=-\sum_{i=-\infty}^{\infty} \frac{k_{x}^{0}}{k_{z 1}^{0} k_{1}} e^{\mathrm{j} k_{z 1}^{0} q} r_{p}^{i}\left(k_{x}^{0}\right), \\
T_{p}^{z}\left(k_{x}, z_{g r}\right)=-\sum_{i=-\infty}^{\infty} \frac{k_{x}^{0}}{k_{z 1}^{0} k_{1}} e^{\mathrm{j} k_{z 1}^{0} q} t_{p}^{i}\left(k_{x}^{0}\right) .
\end{gathered}
$$

Before calculating the Purcell factor, we need to define the energy that the dipole would dissipate in free space $\left(W_{0}\right)$, which is calculated as follows,

$$
W_{0}=\frac{\omega}{2} \operatorname{Im}\left\{\mathbf{p}^{*} \cdot \mathbf{E}_{\mathbf{i}}(\mathbf{0})\right\}=\frac{\omega}{2} \frac{\omega^{2} \mu_{1}}{\sqrt{8 \pi}} p_{z}^{2} \frac{1}{\sqrt{2 \pi}} \operatorname{Re}\left\{\int_{-\infty}^{\infty} \frac{k_{x}^{2}}{k_{z 0} k_{0}^{2}} \mathrm{~d} k_{x}\right\}=\frac{\omega^{3} \mu_{1}}{16} p_{z}^{2} .
$$

The next step consists in calculating the total power dissipated by the dipole under the proposed structure, which is accomplished by substituting (4.62) into (4.60) and then into (4.26), which gives

$$
W=\frac{\omega^{3} \mu_{1}}{8 \pi} p_{z}^{2} \operatorname{Re}\left\{\int_{-\infty}^{\infty}\left[\frac{k_{x}^{2}}{k_{z 1} k_{1}^{2}}+R_{p}^{z}\left(k_{x}, q\right)\left(\mathbf{P}_{\mathbf{p}}^{1}\left(k_{x}\right) \cdot \mathbf{z}\right) \mathrm{e}^{\mathrm{j} k_{z 1} q}\right] \mathrm{d} k_{x}\right\} .
$$

Therefore, the Purcell factor for a 2D perpendicularly polarized dipole $\left(P_{z}^{2 D}\right)$ is calculated as follows,

$$
P_{z}^{2 D}=\frac{W}{W_{0}}=1+\frac{2}{\pi} \operatorname{Re}\left\{\int_{-\infty}^{\infty} R_{p}^{z}\left(k_{x}, q\right)\left[\mathbf{P}_{\mathbf{p}}^{1}\left(k_{x}\right) \cdot \mathbf{z}\right] \mathrm{e}^{\mathrm{j} k_{z 1} q} \mathrm{~d} k_{x}\right\} .
$$

The dipole's total radiated power can be calculated by integrating the far field time averaged Poynting vector over the upper semi-circle. To this end, we need to calculate the electric field generated by a perpendicularly polarized dipole in the far field $\left(\mathbf{E}_{\mathbf{z}}^{\mathbf{F F}}\right)$, which according to the stationary phase method is written as follows,

$$
\mathbf{E}_{\mathbf{z}}^{\mathrm{FF}}(\mathbf{r})=-\mathrm{j} \frac{k_{z 2}}{\sqrt{k_{2}}} E_{t}^{F T}\left(k_{x}, 0\right) \frac{\mathrm{e}^{\mathrm{j} k_{2}|\mathbf{\varrho}|}}{\sqrt{|\mathbf{\varrho}|}} \mathrm{e}^{-j k_{z 2} z_{g r}}=\frac{\omega^{2} \mu_{1}}{\sqrt{8 \pi}} p_{z} \sqrt{k_{2}} \cos \theta T_{p}^{z} \frac{\mathrm{e}^{\mathrm{j} k_{2}|\mathbf{\varrho}|}}{\sqrt{|\mathbf{Q}|}} \mathrm{e}^{-\mathrm{j} k_{2} \cos \theta z_{z^{r}}} \boldsymbol{\theta},
$$

where $\rho$ and $\theta$ are the components of $\boldsymbol{\varrho}$ in cylindrical coordinates (in the $x z$ plane). The following has been assumed for the far field approximation in (4.69),

$$
\mathbf{k}_{2}=\left[\begin{array}{c}
k_{x} \\
0 \\
k_{z 2}
\end{array}\right]=k_{2}\left[\begin{array}{c}
\sin \theta \\
0 \\
\cos \theta
\end{array}\right],-\pi / 2 \leq \theta \leq \pi / 2
$$


The transformation of $\mathbf{E}_{\mathbf{z}}^{\mathbf{F F}}$ in (4.69) into cylindrical coordinates is performed after substituting (4.70) into (4.55), in which gives,

$$
\mathbf{P}_{\mathbf{p}}^{2}\left(\mathbf{k}_{\|}\right)=\frac{k_{z 2}}{k_{2}} \mathbf{x}-\frac{k_{x}}{k_{2}} \mathbf{z}=\boldsymbol{\theta} .
$$

The time-averaged Poynting vector for perpendicular polarized dipole $\left\langle\mathbf{S}_{\mathbf{z}}^{2 \mathbf{D}}\right\rangle$ is given by:

$$
\left\langle\mathbf{S}_{\mathbf{z}}(\mathbf{r})\right\rangle=\frac{1}{2 z_{2}} \operatorname{Re}\left\{\mathbf{E}_{\mathbf{z}}^{\mathrm{FF}} \cdot \mathbf{E}_{\mathbf{z}}^{\mathrm{FF}}\right\} \mathbf{Q}=\frac{\omega^{4} \mu_{1}^{2} k_{2}}{16 \pi z_{2} \rho} p_{z}^{2} \cos ^{2} \theta\left|T_{p}^{z}\right|^{2} \mathbf{Q},
$$

The dipole's total radiated power $\left(Q_{z}^{2 D}\right)$ into medium 2 is then obtained integrating $\left\langle\mathbf{S}_{\mathbf{z}}^{2 \mathbf{D}}\right\rangle$ along the upper semi-circle as follows,

$$
Q_{z}=\int_{-\pi / 2}^{\pi / 2}\left\langle\mathbf{S}_{z}(\mathbf{r})\right\rangle \cdot \varrho \rho d \theta=\frac{\omega^{4} \mu_{1}^{2} k_{2}}{16 \pi z_{2}} p_{z}^{2} \int_{-\pi / 2}^{\pi / 2} \cos ^{2} \theta\left|T_{p}^{z}\right|^{2} \mathrm{~d} \theta
$$

\subsubsection{Parallel polarized dipole}

The dipole moment in this case is oriented along the $x$-axis, thus $\mathbf{p}=p_{x} \mathbf{x}$. After substituting $\mathbf{p}$ into (4.57) and (4.59), the following FTs of the reflected and transmitted electric fields are obtained,

$$
\begin{gathered}
\mathbf{E}_{\mathbf{r}}^{\mathrm{FT}}\left(k_{x}, z\right)=\mathrm{j} \frac{\omega^{2} \mu_{1}}{\sqrt{8 \pi}} p_{x} R_{p}^{x}\left(k_{x}, q\right) \mathbf{P}_{\mathbf{p}}^{1}\left(k_{x}\right) e^{-\mathrm{j} k_{z 1}(z-q)}, \\
\mathbf{E}_{\mathbf{t}}^{\mathrm{FT}}\left(k_{x}, z\right)=\mathrm{j} \frac{\omega^{2} \mu_{1}}{\sqrt{8 \pi}} p_{x} T_{p}^{x}\left(k_{x}, z_{g r}\right) \mathbf{P}_{\mathbf{p}}^{2}\left(k_{x}\right) e^{\mathrm{j} k_{z 1}\left(z-z_{g r}\right)}, \\
R_{p}^{x}\left(k_{x}, q\right)=\sum_{i=-\infty}^{\infty} \frac{1}{k_{1}} e^{\mathrm{j} k_{z 1}^{0} q} r_{p}^{i}\left(k_{x}^{0}\right), \\
T_{p}^{x}\left(k_{x}, z_{g r}\right)=\sum_{i=-\infty}^{\infty} \frac{1}{k_{1}} e^{\mathrm{j} k_{z 1}^{0} q} t_{p}^{i}\left(k_{x}^{0}\right) .
\end{gathered}
$$

The dipole's total dissipated power is calculated after substituting (4.74) into (4.60) and the resulting equations into (4.26), which gives,

$$
W=\frac{\omega^{3} \mu_{1}}{8 \pi} p_{x}^{2} \operatorname{Re}\left\{\int_{-\infty}^{\infty}\left[\frac{k_{z 0}}{k_{0}^{2}}+R_{x}^{z}\left(k_{x}, q\right)\left(\mathbf{P}_{\mathbf{p}}^{1}\left(k_{x}\right) \cdot \mathbf{x}\right) \mathrm{e}^{\mathrm{j} k_{z 1} q}\right] \mathrm{d} k_{x}\right\} .
$$

Therefore, the Purcell factor for a 2D parallel polarized dipole $\left(P_{x}^{2 D}\right)$ is obtained as follows,

$$
P_{x}=\frac{W}{W_{0}}=1+\frac{2}{\pi} \operatorname{Re}\left\{\int_{-\infty}^{\infty} R_{p}^{x}\left(k_{x}, q\right)\left[\mathbf{P}_{\mathbf{p}}^{1}\left(k_{x}\right) \cdot \mathbf{x}\right] \mathrm{e}^{\mathrm{j} k_{z 1} q} \mathrm{~d} k_{x}\right\} .
$$

The dipole's total radiated power is calculated by integrating the far field Poynting vector temporal mean over the upper semi-circle. To this end, we need to calculate the electric 
field generated by a parallel polarized dipole in the far field $\left(\mathbf{E}_{\mathbf{x}}^{\mathbf{F F}}\right)$ which, according to the stationary phase method, is given by,

$$
\mathbf{E}_{x}^{\mathrm{FF}}(\mathbf{r})=-\mathrm{j} \frac{k_{z 2}}{\sqrt{k_{2}}} E_{t}^{F T}\left(k_{x}, 0\right) \frac{\mathrm{e}^{\mathrm{j} k_{2}|\mathbf{\varrho}|}}{\sqrt{|\mathbf{\varrho}|}} \mathrm{e}^{-j k_{z 2} z_{z^{r}}}=\frac{\omega^{2} \mu_{1}}{\sqrt{8 \pi}} p_{x} \sqrt{k_{2}} \cos \theta T_{p}^{x} \frac{\mathrm{e}^{\mathrm{j} k_{2}|\mathbf{\varrho}|}}{\sqrt{|\mathbf{\varrho}|}} \mathrm{e}^{-\mathrm{j} k_{2} \cos \theta z_{z^{r}}} \boldsymbol{\theta},
$$

Equation (76) gives the electric fields in cylindrical coordinates $(\mathbf{Q}, \boldsymbol{\theta})$. The time-averaged Poynting vector and the dipole's total radiated power $\left(\left\langle\mathbf{S}_{\mathbf{x}}^{\mathbf{2 D}}\right\rangle\right.$ and $Q_{x}^{2 D}$, respectively) are calculated as follows,

$$
\begin{gathered}
\left\langle\mathbf{S}_{\mathbf{x}}(\mathbf{r})\right\rangle=\frac{1}{2 z_{2}} \operatorname{Re}\left\{\mathbf{E}_{\mathbf{x}}^{\mathrm{FF}} \cdot \mathbf{E}_{\mathbf{x}}^{\mathrm{FF}}\right\} \mathbf{Q}=\frac{\omega^{4} \mu_{1}^{2} k_{2}}{16 \pi z_{2} \rho} p_{x}^{2} \cos ^{2} \theta\left|T_{p}^{x}\right|^{2} \mathbf{Q}, \\
Q_{x}=\int_{-\pi / 2}^{\pi / 2}\left\langle\mathbf{S}_{\mathbf{x}}(\mathbf{r})\right\rangle \cdot \mathbf{Q} \rho \mathrm{d} \theta=\frac{\omega^{4} \mu_{1}^{2} k_{2}}{16 \pi z_{2}} p_{x}^{2} \int_{-\pi / 2}^{\pi / 2} \cos ^{2} \theta\left|T_{p}^{x}\right|^{2} \mathrm{~d} \theta
\end{gathered}
$$




\subsection{Optimization and analysis}

The proposed method is now applied to the analysis of a HMM composed of 8 alternating layers of $\mathrm{Ag}(\varepsilon=-25+1.71 \mathrm{j})$ and $\mathrm{TiO}_{2}(\varepsilon=6.38)$ [37], as seen in the Chapter 3 (see Figure 3.2). The $\mathrm{Ag}$ and $\mathrm{TiO}_{2}$ layer thicknesses are, respectively, $10 \mathrm{~nm}$ and $25 \mathrm{~nm}$, except for the bottom $\mathrm{Ag}$ and top $\mathrm{TiO}_{2}$ layers, whose thicknesses are $5 \mathrm{~nm}$ and $10 \mathrm{~nm}$, respectively. Since the goal of this Chapter is to increase the system quantum efficiency by means of decoupling the high- $k$ modes of the structure, we have added an Ag grating on the top of the HMM, as can be seen in Figure 4.3. In this configuration, the QE (Cy7) are placed embedded in a semi-finite PMMA $(\varepsilon=2.21)$ bottom layer, as seen in Figure 4.3. In practice, the PMMA layer will be deposited on the top of a $\mathrm{SiO}_{2}$ substrate $(\varepsilon=2.16)$. However, the small mismatch between those layers drastically reduces the reflection $(R<1 \%)$, and consequently, we can assume a semi-infinity PMMA layer and apply the set of equations proposed in subsection 4.1.

The analysis starts with the HMM's dispersion curve obtained for the TM and TE polarizations [101], and shown in Figure 4.4 (b). The real part (hollow squares) of the dispersion curve for the TE polarized wave is close to zero, while the imaginary part (hollow circle) is high and increases with $k_{x} / k_{0}$ (essentially a metal-like behavior). In contrast, the real part (solid squares) of the dispersion for the TM polarized wave presents a hyperbolic profile in the range $2.75<\left|k_{x} / k_{0}\right|<10$. In the same region, the imaginary part (solid circles) is close to zero, thus producing low-loss wave propagation. Above the

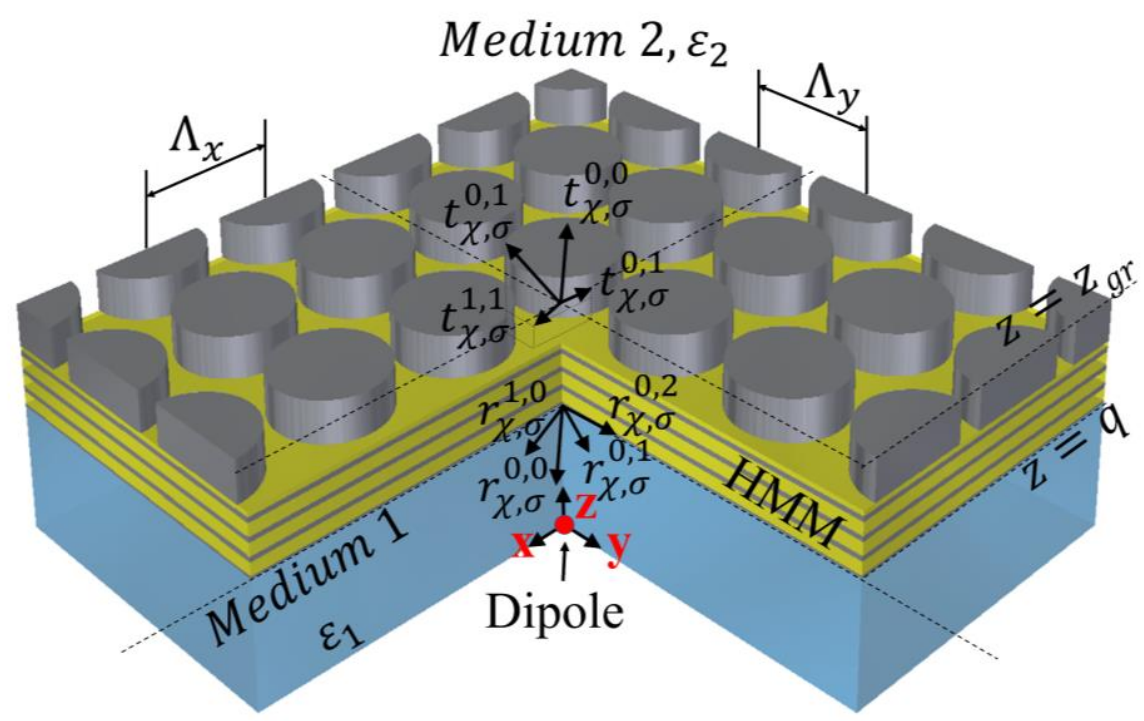

Figure 4.3. Proposed geometry in which the QEs are modelled as a dipole embedded in Medium 1 (with permittivity $\varepsilon_{1}$ ), centered at the origin and at a distance $q$ from the HMM bottom surface. The gratings with periodicity $\Lambda_{x}$ and $\Lambda_{y}$ in $\mathrm{x}$ and $\mathrm{y}$ directions, respectively, are on top of the HMM and are covered with Medium 2 (with permittivity $\varepsilon 2)$. 
upper cut-off $\left(\left|k_{x} / k_{0}\right|=10\right)$, the real part becomes flat, the imaginary part becomes large and, as a result, waves cannot propagate in this region. The HMM bulk modes have $k_{x} / k_{0}>1$ and therefore do not couple to the air (their energy is dissipated inside the HMM). These high- $k$ modes can be decoupled from the HMM into the air by means of a metallic grating with period $\Lambda x$, fill factor $f f$, and height $a$, located on top of the HMM. The effect of the grating on the device performance is discussed next.

\subsubsection{D Optimization procedure}

The optimization of the grating parameters is carried out based on the 2D model, as discussed earlier and shown in Figure 4.4 (a). The primary parameter to be adjusted is $\Lambda x$, since it affects the grating momentum and, consequently, changes the 2D-dipole's emission direction. For this adjustment, we calculate the modulus of the time averaged Poynting vector for perpendicular $\left(\mathbf{S}_{\mathbf{z}}^{\mathbf{2 D}}\right)$ and parallel $\left(\mathbf{S}_{\mathbf{x}}^{\mathbf{2 D}}\right)$ polarization as function of $\Lambda_{x}$ and $\theta$ using (4.72) and (4.81), assuming the gratings' $f f=50 \%, a=100 \mathrm{~nm}$, and $q=10 \mathrm{~nm}$ [55], [57]. The 2D-dipole is aligned with the middle of the groove, as shown in Figure 4.4 (a). The normalized $\mathbf{S}_{\mathbf{z}}^{\mathbf{2 D}}$ and $\mathbf{S}_{\mathbf{x}}^{\mathbf{2 D}}$ are shown in Figure 4.5 (a) and (b), respectively. These figures show that the radiation pattern is highly influenced by the grating period. The emission angle can be tuned in the full range of $\theta$ by changing the period from 280 to 460 $\mathrm{nm}$. Moreover, at $420 \mathrm{~nm}$ the radiation is highly directive, and the emission peak is normal to the HMM surface. This point is particularly interesting for applications that demand light coupling into another device, such as fibers or sensors.

Even though $\mathbf{S}_{\mathbf{z}}^{2 \mathbf{D}}$ and $\mathbf{S}_{\mathbf{x}}^{2 \mathbf{D}}$ provide valuable information about the beam directivity, it is important to calculate how much power is radiated by the 2D-dipole $\left(Q_{\alpha}^{2 D}\right)$ and the Purcell factor $\left(P_{\alpha}^{2 D}\right)$. The 2D-dipole's radiated power is normalized to the total power it would radiate in free space $\left(W_{0}^{2 D}\right), \Psi_{\alpha}^{2 D}=Q_{\alpha}^{2 D} / W_{0}^{2 D}$, with $W_{0}^{2 D}$ calculated using (4.66). Figure 4.5 (c) and (d) show $\Psi_{\alpha}^{2 D}$ and $P_{\alpha}^{2 D}$ for perpendicularly (red line) and parallel (blue line) polarized 2D-dipoles at $q=10 \mathrm{~nm}$. These figures also show the $2 \mathrm{D}$ results obtained by using

(a)

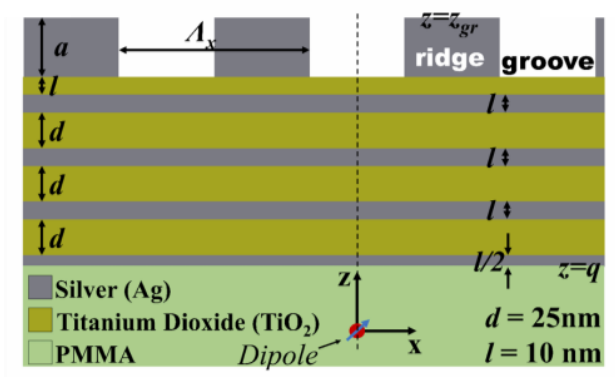

(b)

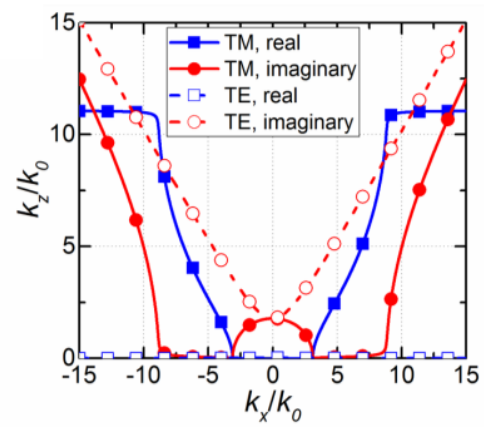

Figure 4.4. (a) Silver Gratings with period $\Lambda x$ and height $a$ on top of a HMM composed of 8 alternating layers of $\mathrm{Ag}(\varepsilon=-25+1.71 \mathrm{j})$ and $\mathrm{TiO}_{2}(\varepsilon=6.38)$. (b) Real (squares) and imaginary (circles) parts of the HMM dispersion curve for TM (solid symbols) and TE polarization (hollow symbols). 
a commercial-grade simulator based on the FDTD method [151] (squares and circles for perpendicular and parallel polarization, respectively). The FDTD results show good agreement with those obtained with the proposed approach, confirming the accuracy of our model. For small periods $\left(\Lambda_{x}<70 \mathrm{~nm}\right)$, the grating behaves as a homogenous material, which explains the smooth variation of $\Psi_{\alpha}^{2 D}$ and $P_{\alpha}^{2 D}$. As the period increases, the grating begins to interfere with the high- $k$ modes. Even slight changes in $\Lambda x$ changes significantly the mode coupling, and both $\Psi_{\alpha}^{2 D}$ and $P_{\alpha}^{2 D}$ exhibit fast variations. The fast oscillations smooth out for $\Lambda_{x}>260 \mathrm{~nm}$, and the power radiated by the 2D-dipole is maximum for perpendicular polarization at $345 \mathrm{~nm}$, where $\Psi_{z}^{2 D}=1.1$. In the same region $\left(\Lambda_{x}>260 \mathrm{~nm}\right)$, the Purcell factor is approximately constant at 19 for perpendicular and 16 for parallel polarization. By increasing the period even further $\left(\Lambda_{x}>475 \mathrm{~nm}\right)$, the normalized power decreases to below 0.75 for both polarizations. Moreover, by choosing the period in the interval $260 \mathrm{~nm}<\Lambda_{x}<475 \mathrm{~nm}$ it is possible to obtain both a highly directive beam and a high $\Psi_{\alpha}^{2 D}$ and $P_{\alpha}^{2 D}$. Although $\Psi_{x}^{2 D}$ peaks at $\Lambda_{x}=345 \mathrm{~nm}$, we choose $\Lambda_{x}=420 \mathrm{~nm}$ because the emission is almost normal to the surface and the normalized power is still high, $\Psi_{z}^{2 D}=0.94$.

The second parameter to be optimized is the grating fill factor Figure 4.6 (a) shows the Purcell factor (hollow symbols) and normalized radiated power (full symbols) for perpendicular (squares) and parallel (circles) polarization calculated by varying the grating fill factor from $5 \%$ to $95 \%$. The maximum normalized power radiated by the $2 \mathrm{D}$ -
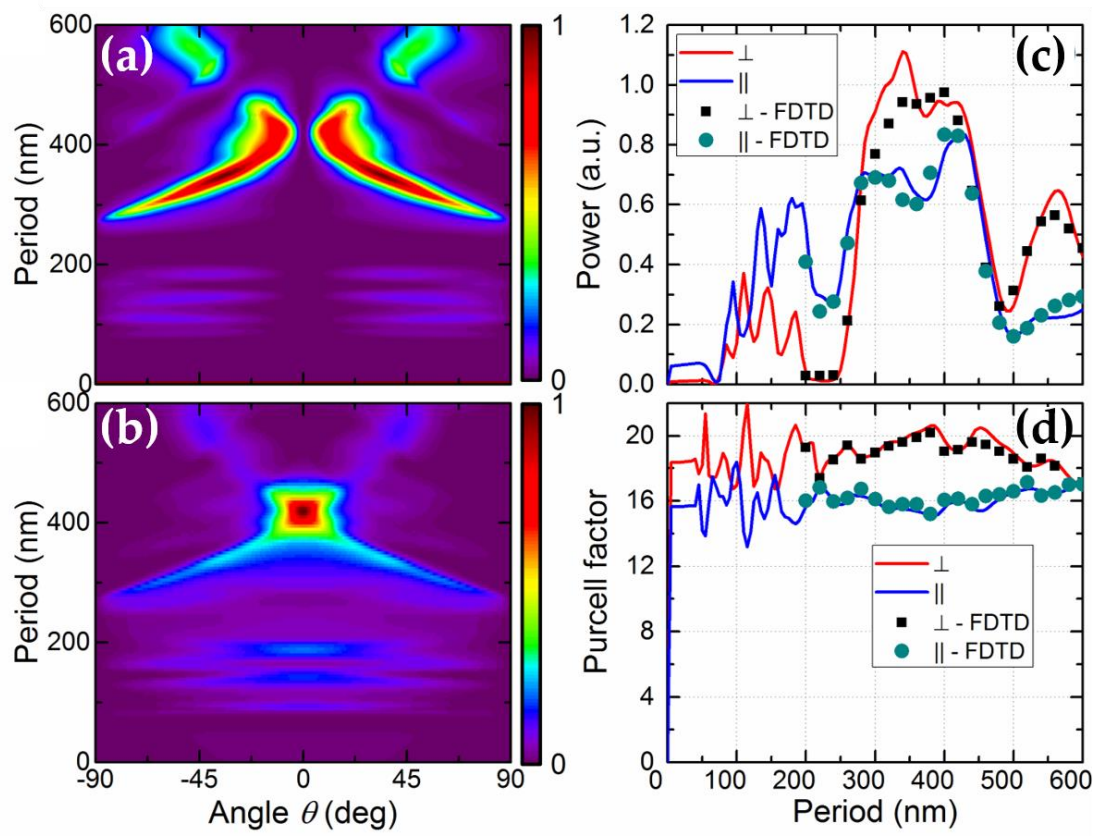

Figure 4.5. (a) Perpendicularly and (b) parallel polarized normalized emission patterns of a 2Ddipole as function of the grating period. (c) Normalized power radiated by the 2Ddipole $\left(\Psi_{\alpha}^{2 D}\right)$ and (d) Purcell factor, both for a 2D-dipole perpendicularly (red curve) and parallel (blue curve) polarized. The $2 \mathrm{D}$ results obtained with a Lumerical FDTD for perpendicularly (squares) and parallel (circles) polarized 2D-dipoles are also shown in (c) and (d) for the sake of comparison. 
dipole occurs at $f f=70 \%\left(\Psi_{z}^{2 D}=1.43\right)$ for perpendicular and at $f f=47 \%\left(\Psi_{x}^{2 D}=0.78\right)$ for parallel polarization. The Purcell factor for perpendicular polarization is higher than that for parallel polarization at ff $>35 \%$. Most of the electric field is reflected in the first layers of the HMM, reducing the influence of the grating fill factor on the Purcell factor, as seen in Figure 4.6 (a). Since the goal is to decrease the lifetime of the QEs and yet to increase the radiation power, the best scenario is present when $f f=70 \%$, in which the $2 \mathrm{D}$-dipole that radiates more power also has the higher Purcell factor.

The only parameter yet to be optimized is the grating height $a$. For that, we adopt $\Lambda_{x}=420$ $\mathrm{nm}, f f=70 \%$, and vary the height from 5 to $200 \mathrm{~nm}$. The Purcell factor (hollow symbols) and the normalized radiated power (full symbols) for perpendicular (squares) and parallel (circles) polarization are shown in Figure 4.6 (b). The Purcell factor for perpendicular and parallel polarizations present a slight variation when the grating height is changed. This is caused by the electric field being reflected mostly at the first layers of the HMM. As a result, the grating has little contribution to the reflected electric field. However, the transmitted field is highly affected by the grating. High- $k$ modes interact only weakly with a thin grating, causing the diffraction efficiency to decrease. However, a thick grating causes the modes to become more attenuated by the metal, consequently decreasing the transmission coefficients. Therefore, the 2D-dipole radiation power is low either for very thin or for thick grating, as shown in Figure 4.6 (b). The optimum value is present at an intermediate thickness of $a=80 \mathrm{~nm}$, at which the perpendicular 2D-dipole has a normalized radiated power of $\Psi_{Z}^{2 D}=1.67$ and $P_{Z}^{2 D}=20$.

Once the grating's geometrical parameters have been optimized $\left(\Lambda_{x}=420 \mathrm{~nm}, f f=70 \%\right.$ and $a=80 \mathrm{~nm}$ ), we can next calculate the normalized squared electric field of a 2D-dipole. This is carried out using (4.72) and (4.81). The normalized squared electric field and emission pattern for perpendicular polarization are both shown in Figure 4.7 (a). The emission pattern presents two symmetrical lobes oriented along $\theta= \pm 6^{\circ}$ with gain $G=13.9 \mathrm{~dB}$. In addition, each lobe of the propagating wave has a full width at half maximum FWHM $=6^{\circ}$.

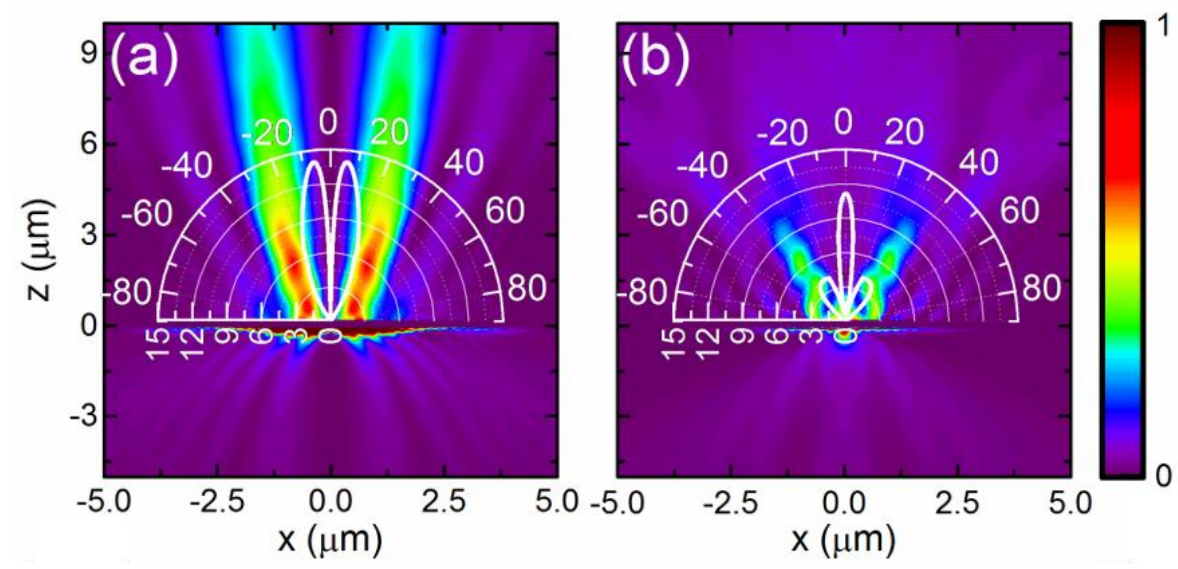

Figure 4.7. Normalized squared electric field and emission pattern of a perpendicular (a) and parallel (b) polarized 2D-dipoles. 
In contrast, the emission pattern for parallel polarization, shown in Figure 4.7 (b) along with its normalized squared electric field, presents a main lobe at $\theta=0^{\circ}$ with $\mathrm{G}=11.1 \mathrm{~dB}$ and $\mathrm{FWHM}=5^{\circ}$, along with two secondary lobes. In summary, the electric field emitted by the 2D-dipole propagates with high gain nearly normal to the surface. The polarization with the higher Purcell factor (perpendicular) has, as predicted, most of its power radiating into medium 2, contributing to the increase of the overall Purcell factor.

The next step is to determine $\Psi_{\alpha}^{2 D}$ and $P_{\alpha}^{2 D}$ as function of position. This is accomplished here by allowing the $2 \mathrm{D}$-dipole position to be shifted in the reflection and transmission coefficient expressions as follows,

$$
v_{\chi, \sigma}^{i, m}\left(\mathbf{k}_{\|}, \Delta x, \Delta y\right)=v_{\chi, \sigma}^{i, m}\left(\mathbf{k}_{\|}, 0,0\right) e^{-\mathrm{j}\left(i K_{x} \Delta x+m K_{y} \Delta y\right)},
$$

where $\Delta x$ and $\Delta y$ are the incremental shifts in $x$ and $y$ axes, respectively. A 2D grating exhibits translation only in $x$-axis, thus $K_{y}=0$. Note that $v_{\chi, \sigma}^{i, m}$ has period $\Lambda_{x}$ and $\Lambda_{y}$ along the $x$ and $y$ axes, respectively. The expression for the Purcell factor can now be obtained by substituting (4.83) into (4.62) and (4.63) [(4.74) and (4.75)], and the resulting expressions into (4.68) [(4.79)] for perpendicular [parallel] polarized dipole. The Purcell factor is calculated for perpendicularly and parallel polarized 2D-dipole s located at a distance $q$ from the HMM bottom surface and whose position varied in the interval $-\Lambda_{x} / 2$

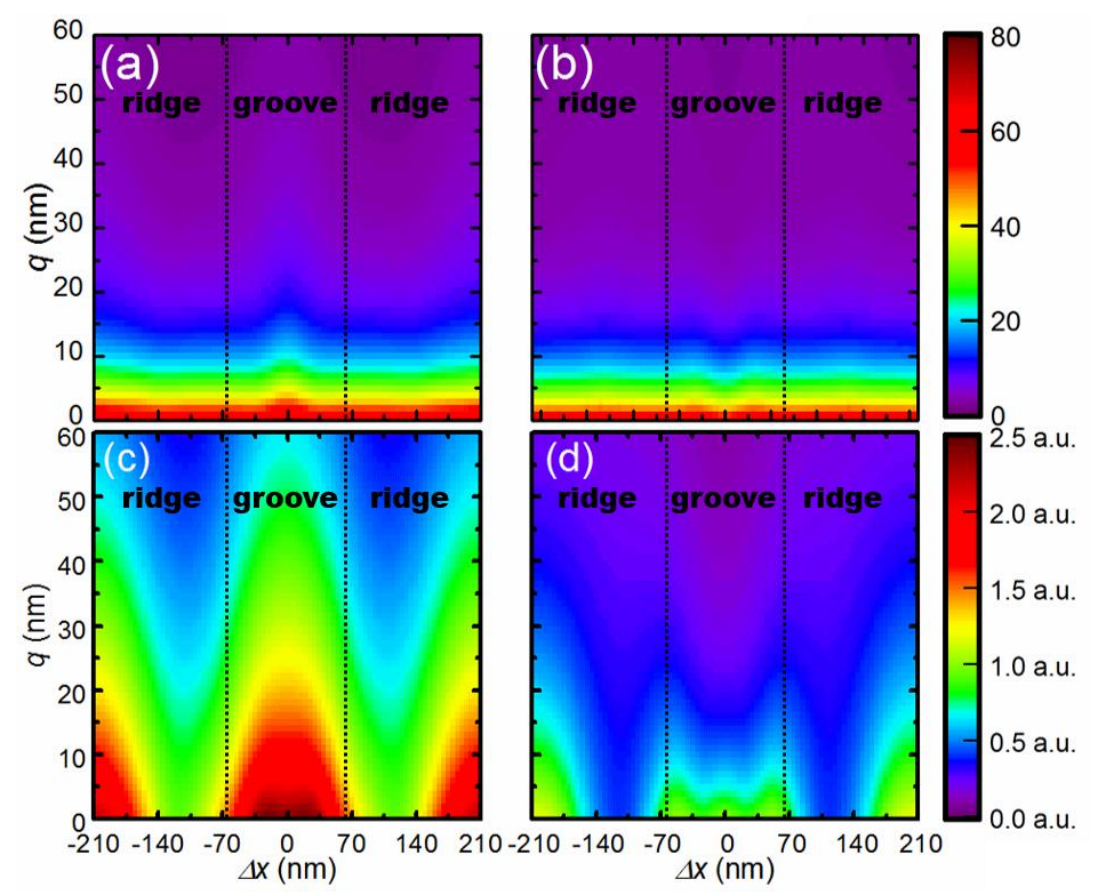

Figure 4.8. Left and right columns refer to perpendicular and parallel polarized 2D-dipoles, respectively. Purcell factor $(a, b)$ and normalized radiated power $(c, d)$ calculated as function of the 2D-dipole position. (a,c) Perpendicular and (b,d) parallel polarization. The colorbar is valid for both figures of each row. 
to $\Lambda_{x} / 2$. The results for both 2D-dipole polarizations are shown in Figure 4.8 (a) and (b). Note that the Purcell factor increases exponentially as the 2D-dipole approaches the HMM surface, which is due to the coupling of the 2D-dipole's evanescent fields to the HMM bulk modes. The reverse is true if the 2D-dipole moves away from the HMM surface because of the exponential decay of these fields. The HMM bottom layers (closer to the 2D-dipole) are where most of the 2D-dipole's evanescent fields are reflected. However, part of the energy corresponding to these fields reaches the grating, resulting in a slight dependence of the Purcell factor on the dipole position as it moves along the grating groove to ridge in the $x$-axis.

The next parameter to be examined is the normalized radiated power obtained here as function of $q$ and $\Delta x$, and shown in Figure 4.8 (c) and (d) for perpendicular and parallel polarizations, respectively. These figures show that the closer the $2 \mathrm{D}$-dipole gets to the HMM, the higher $\Psi_{\alpha}^{2 D}$ is. This behavior confirms that the grating is in fact converting the $2 \mathrm{D}$-dipole's high- $k$ modes coupled to the HMM into propagating waves in medium 2 . As the 2D-dipole moves away from the HMM surface, the high- $k$ modes' power exponentially decreases causing a decrease on the power coupled to the far field. In contrast to the Purcell factor, the radiated power changes significantly when the 2Ddipole is shifted along the $x$-axis. According to Figure 4.8 (c) and (d), the 2D-dipole radiates more power if located under the groove's or ridge's center.

\subsubsection{D Configuration: Analysis and Discussion}

With the grating optimization complete, the next step consists in converting the 2D structure into a 3D one. The most intuitive way of doing this would be using a Bullseye configuration for the grating [39], [57]. However, this configuration produces high $P_{\alpha}$ and $Q_{\alpha}$ only for dipoles located in the center of the Bullseye. Since in the proposed structure the QE's are uniformly distributed inside the PMMA, the Bullseye configuration is not suitable for our application. For this reason, we choose a grating consisting of a periodic array of nano-cylinders to represent the 3D model, as depicted in Figure 4.3. The periods along $x$ and $y$ axes are $420 \mathrm{~nm}$ and $a=80 \mathrm{~nm}$. Note that the change from 2D to 3D requires the fill factor $(f f=70 \%)$ to be squared, thus the cylinder radius $(w)$ is calculated to provide a fill factor of $49 \%$, corresponding to $w=170 \mathrm{~nm}$. The HMM geometrical parameters are the same for both dimensions, as shown in Figure 4.4 (a).

Before analyzing the 3D HMM, we provide additional validation of the 2D model. Bear in mind that the use of 2D simulations for optimizing electromagnetic devices is widely used in the literature due to their much lower computational cost compared to 3D ones. Although 2D simulations do not fully describe the 3D behavior, they provide key insights for the optimization process. In this sense, we have performed three 3D-FDTD simulations with the same structure illustrated in Figure 4.3 but with different fill factors $(f f)$, for the optimum case $(f f=49 \%)$ and two alternatives scenarios, $f f=30 \%$ and $70 \%$. Since our structure is optimized for perpendicular polarization, we have considered a dipole perpendicularly oriented. Figure 4.9 shows the total power radiated to the far field by the perpendicular dipole for $f f=30 \%$ (circles), $49 \%$ (squares) and $70 \%$ (triangles). As this figure 


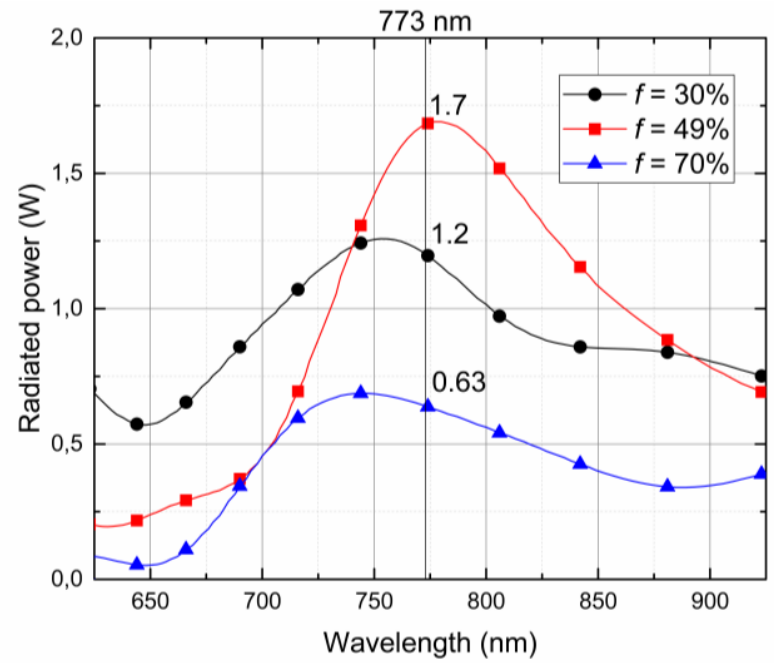

Figure 4.9. 3D-FDTD simulations of the total power radiated to the far field by the perpendicular dipole for $f=30 \%$ (circles), $49 \%$ (squares) and $70 \%$ (triangles).

shows, in addition to radiating more power $(1.7 \mathrm{~W})$, the structure with the optimized fill factor $(f f=49 \%)$ outperforms all others at the operating wavelength $(\lambda=773 \mathrm{~nm})$. These results indicate that the optimization carried out in $2 \mathrm{D}$ can indeed provide good geometrical parameters to be used in the 3D model.

After the validation of our 2D optimization procedure, we calculated the radiation pattern emission on the plane $z=z_{0}+z_{g r}$ of a perpendicularly and parallel polarized QE placed at $q$ $=10 \mathrm{~nm}$. Figure 4.10 shows the normalized Poynting vector modulus $(\mathrm{a}, \mathrm{d})$ and electric field decomposed in p- $(b, e)$ and s-polarization $(c, f)$ for a perpendicularly $(a-c)$ and parallel (d-f) polarized QE. According to Figure 4.10 (a) and (d), the dipole emission is not as directional as in the $2 \mathrm{D}$ case. Nevertheless, $50 \%$ of the radiated power is within the $30^{\circ}$ cone for both perpendicular and parallel polarizations. The loss of directivity (compared to the 2D model) is mainly due to the cross polarized terms in (4.22) and (4.35). On one hand, perpendicularly polarized dipoles do not radiate s-polarized waves, therefore the s-polarized electric field shown in Figure 4.10 (c) is generated by the crosspolarization terms in (4.22). The peaks in the radiation pattern for p-polarized waves (Figure 4.10 (b)) are inside the $20^{\circ}$ cone, while the peaks for s-polarized waves are in the $55^{\circ}$ cone (Figure 4.10 (c)). On the other hand, parallel polarized dipoles radiate both $\mathrm{p}$ and s-polarized waves. However, s-polarized waves are highly attenuated by the HMM, and consequently do not have a considerable influence on the transmitted fields. As a result, the s-polarized waves are mostly generated by the cross-polarization term $t_{s, p}^{i, m}$ in (4.35). Note once again that the peaks of the p-polarized waves (Figure 4.10 (e)) are in a smaller cone compared to the s-polarized waves (Figure 4.10 (f) $)\left(10^{\circ}\right.$ and $30^{\circ}$ for $\mathrm{p}$ - and s-polarized waves, respectively).

Figure 4.11 (a) and (b) show the normalized dissipated power density ( $D_{\alpha}=$ $k_{0}^{2} \partial^{2} P_{\alpha} / \partial k_{x} \partial k_{y}$ ) for perpendicular and parallel polarizations, respectively, with $p$ chosen 

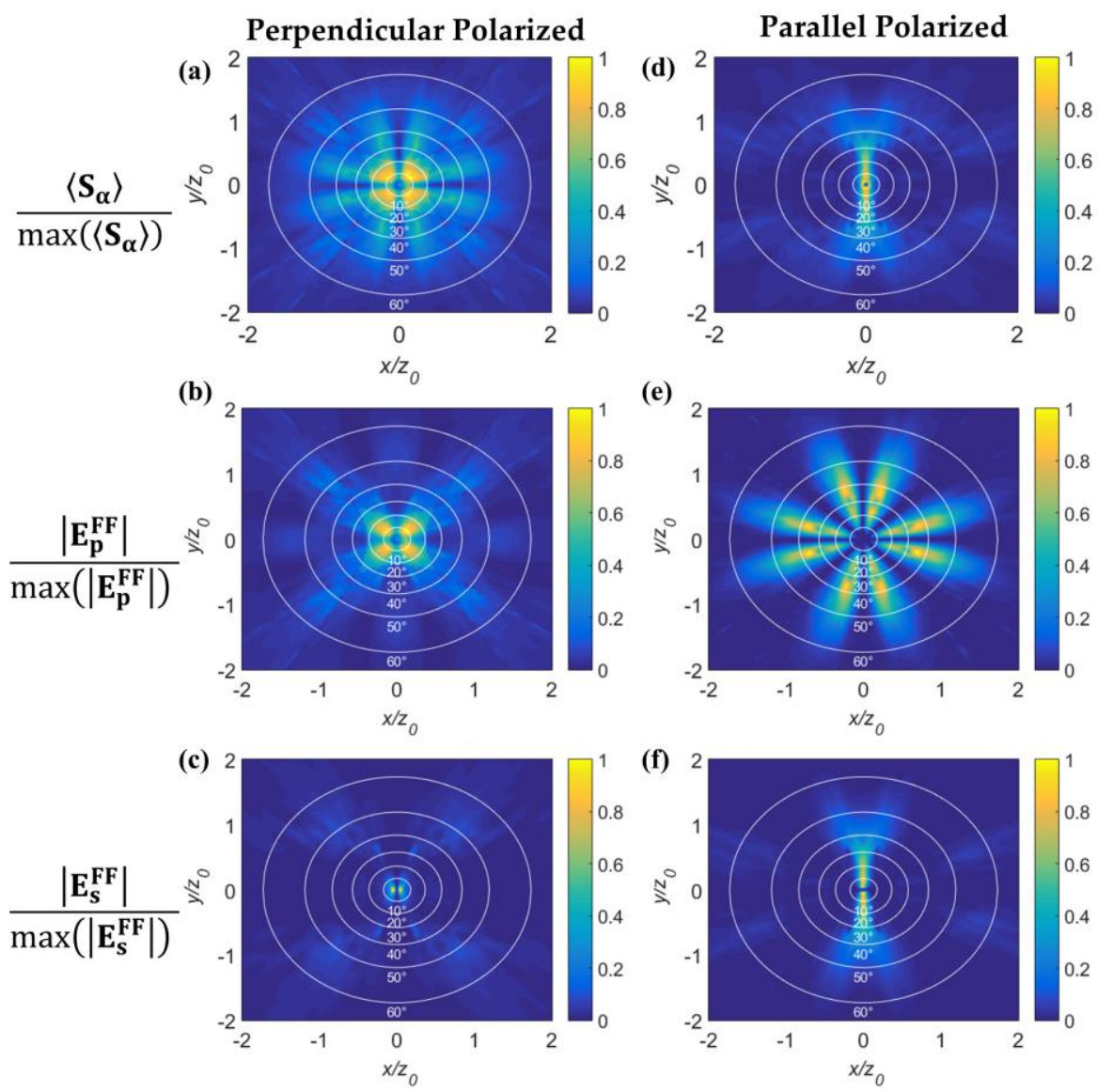

Figure 4.10. Normalized Poynting vector of a perpendicularly (a) and parallel (d) polarized dipole placed at $q=10 \mathrm{~nm}$. Normalized electric fields emitted by a perpendicularly $(\mathrm{b}, \mathrm{c})$ and parallel $(\mathrm{e}, \mathrm{f})$ polarized dipole decomposed in $p$ - $(\mathrm{b}, \mathrm{e})$ and $s$-polarization $(\mathrm{c}, \mathrm{f})$.

so that the dipole radiates $1 \mathrm{~W}$ in free space. According to (4.12), the perpendicular dipole emits only $p$-polarized waves whose amplitudes depend on $k_{/ /}$. It should be noted that without the grating this dependence would have produced circles in the $D_{z}$ map. However, according to (4.24), (4.37) and (4.38), the grating changes the waves' momenta causing the dependence of $k_{/ /}$to be changed to $k_{x}$ and $k_{y}$ and therefore deforming these circles, as shown in Figure 4.11 (a). As mentioned before, the bottom HMM layers are mainly responsible for wave reflection, therefore the $D_{z}$ map deformations are small and the circles' pattern can still be seen in Figure 4.11 (a). In addition, the power is dissipated inside the circle $k_{/ /} / k_{0}<10$, which is the region where the medium behaves as a HMM (see Fig. 2 (b)). The parallel polarized dipole radiates TE and TM waves which are proportional to $k_{\|}$, but modulated by $\sin (\psi)$ and $\cos (\psi)$, respectively $\left(\psi=\operatorname{atan}\left(k_{y} / k_{x}\right)\right)$. Since only TM modes couple to the HMM, the $D_{x}$ map also presents deformed circles modulated by $\cos (\psi)$, as can be seen in Figure 4.11 (b). 

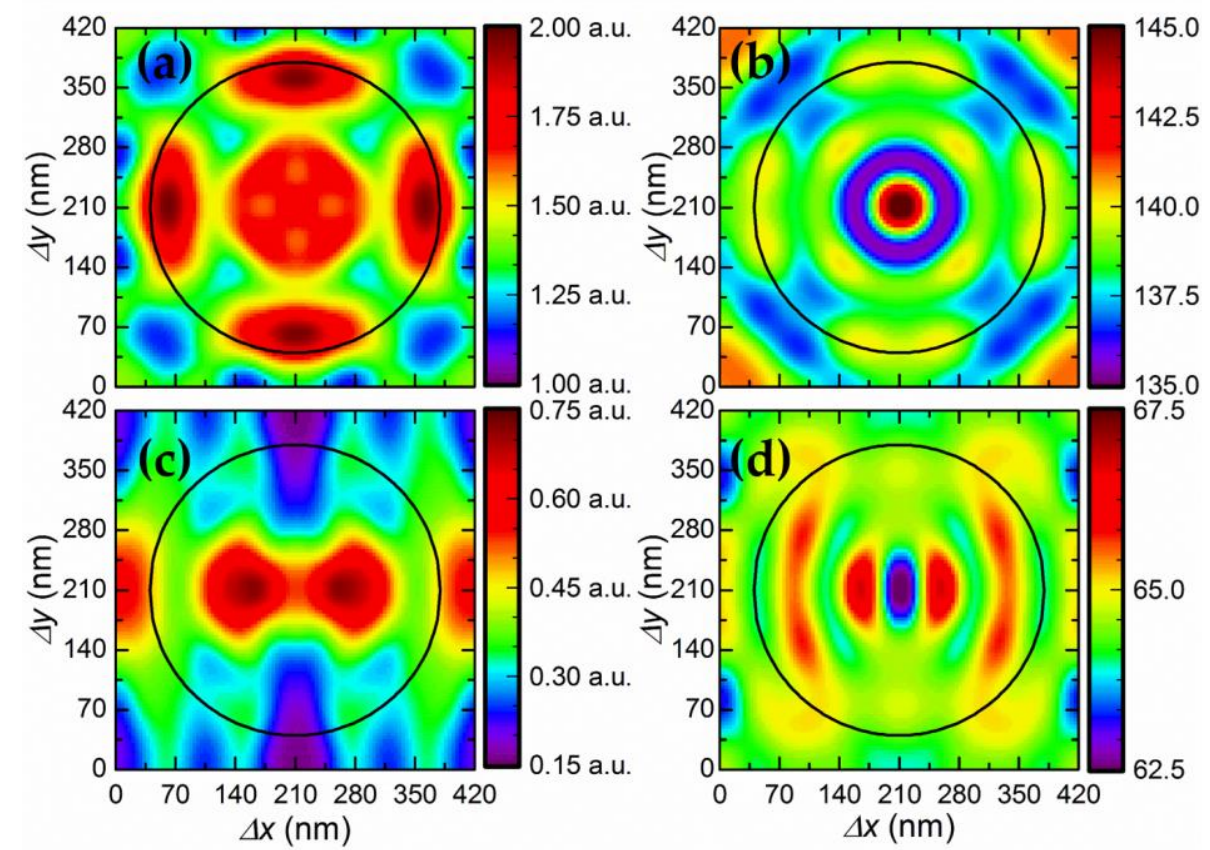

Figure 4.12. Normalized radiated power $(a, c)$ and Purcell factor $(b, d)$ of a perpendicularly $(a, b)$ and parallel (c,d) polarized dipole as function of its position variation $\Delta x$ and $\Delta y$ at $q$ $=10 \mathrm{~nm}$. The solid black line in $(\mathrm{a}-\mathrm{d})$ represents the periodic array of nano-cylinders edges.

Next, we calculate $P_{\alpha}$ and the normalized radiated power $\left(\Psi_{\alpha}^{3 D}=Q_{\alpha} / W_{0}\right)$ as functions of $\Delta x, \Delta y$, and $q$. Figure 4.12 (a) and (c) show $\Psi_{\alpha}^{3 D}$ for perpendicular and parallel polarizations, respectively, at $q=10 \mathrm{~nm}$. The corresponding Purcell factor is shown in Figure 4.12 (b) and (d) for perpendicular and parallel polarization, respectively. As noted before, in contrast to TE modes, TM modes strongly couple to the HMM bulk modes. Moreover, the perpendicular dipole, which radiates only TM waves, has a $\Psi_{\alpha}^{3 D}$ higher than that of parallel dipoles, which radiates both TE and TM waves. This behavior is observed for any dipole position, see Figure 4.12 (b) and (d). A shift of the dipole in the $x y$-plane causes a large variation on both $\Psi_{z}^{3 D}$ and $\Psi_{x}^{3 D}$, with the dipoles radiating more power when positioned below the cylinder edge for perpendicular polarization (up to 2) and below the cylinder center for parallel polarization (up to 0.75). In contrast, the dipole's position in $x y$ plane has little influence on $P_{\alpha}$, changing it only by a factor of $7.1 \%$ for both polarizations. This is a consequence of the dipole's radiated waves being mostly reflected by the HMM, reducing the grating influence on the Purcell factor. As observed in Figure $4.10(\mathrm{a}, \mathrm{c})$, the dipole dissipates more power for perpendicular polarization due to the strong coupling to TM bulk modes. As a result, the Purcell factor at any position is higher for this orientation, as shown in Figure $4.12(\mathrm{~b}, \mathrm{~d})$. For instance, at $q=10 \mathrm{~nm}$ and $\Delta y=\Delta x=0, P_{z}=145$ and $P_{x}=62$. Similarly to the 2D case, $P_{\alpha}$ and $Q_{\alpha}$ is higher for dipoles closer to the HMM surface, but it exponentially decays as the separation increases due to evanescent coupling to TM bulk modes. In summary, our approach allows both $P_{\alpha}$ and 
$\Psi_{\alpha}^{3 D}$ to be mapped as function of the dipole's position. This is highly desirable as it allows us to understand how the QE location affects its behavior.

\subsection{Comparison between HMM with and without metallic gratings}

After generating the $P_{\alpha}$ and $\Psi_{\alpha}^{3 D}$ maps as function of the dipole's position, we can now calculate the system's $g(t)$. The goal is to compare this parameter from the optimized grating assisted structure proposed in this chapter (HMM_opt) with that of the plain HMM proposed in chapter 3 (HMM_ch3). In case of HMM_ch3, $g(t)$ is calculated with the help of (3.16) and (3.17). However, for HMM_opt, both $Q_{\alpha}$ and $P_{\alpha}$ change with the QE's position ( $\left.\mathbf{r}_{0}\right)$, as seen in Figure 4.12. Consequently, (3.16) and (3.17) need to be modified to take these effects into account. Assuming the QEs are uniformly distributed, $g(t)$ can be calculated as follows [114],

$$
\begin{gathered}
g(t)=\int_{0}^{\Lambda_{x}} \int_{0}^{\Lambda_{y}} \int_{0}^{h} \frac{\left(\Upsilon_{x} \eta_{x}\left(\mathbf{r}_{\mathbf{0}}\right) e^{-P_{x}\left(\mathbf{r}_{\mathbf{0}}\right) t}+\Upsilon_{y} \eta_{y}\left(\mathbf{r}_{\mathbf{0}}\right) e^{-P_{y}\left(\mathbf{r}_{\mathbf{0}}\right) t}+\Upsilon_{z} \eta_{z}\left(\mathbf{r}_{\mathbf{0}}\right) e^{-P_{z}\left(\mathbf{r}_{\mathbf{0}}\right) t}\right)}{h \eta_{\text {eff }}} \mathrm{d} z \mathrm{~d} y \mathrm{~d} x, \\
\eta_{\text {eff }}=\int_{0}^{\Lambda_{x}} \int_{0}^{\Lambda_{y}} \int_{0}^{h} \frac{\left(\Upsilon_{x} \eta_{x}\left(\mathbf{r}_{\mathbf{0}}\right)+\Upsilon_{y} \eta_{y}\left(\mathbf{r}_{\mathbf{0}}\right)+\Upsilon_{z} \eta_{z}\left(\mathbf{r}_{\mathbf{0}}\right)\right)}{h} \mathrm{~d} z \mathrm{~d} y \mathrm{~d} x,
\end{gathered}
$$

where $\Upsilon_{\alpha}$ is the probability of the QE being polarized along the $\alpha$-axis, $\eta_{\alpha}=\Psi_{\alpha}^{3 D} / P_{\alpha}$ is the quantum efficiency of the QE polarized along the $\alpha$-axis, $\eta_{\text {total }}$ is the system's quantum efficiency. After calculating $g(t)$, the system's lifetime $(\tau)$ is estimated as $g(\tau)=1 / \mathrm{e}$, resulting in the effective enhancement of spontaneous emission rate $P_{\text {eff }}=1 / \tau$. We carry out the comparison between both structures (i.e, between HMM_opt and HMM_ch3), investigating three scenarios:

I. All QEs are perpendicularly polarized $\left(\Upsilon_{z}=1 ; \Upsilon_{x}=\Upsilon_{y}=0\right)$, with $P_{\text {eff }}^{z}=P_{\text {eff }}$ and $\eta_{\text {eff }}^{Z}=\eta_{\text {eff }}$;

II. All the QEs are parallel polarized $\left(\Upsilon_{x}=1 ; \Upsilon_{z}=\Upsilon_{y}=0\right)$. Note that the results would be the same if we consider $\left(\Upsilon_{y}=1 ; \Upsilon_{z}=\Upsilon_{x}=0\right)$. $P_{\text {eff }}^{x}=P_{\text {eff }}$ and $\eta_{\text {eff }}^{x}=$ $\eta_{\text {eff }}$;

III. The QEs are equally polarized $\left(\Upsilon_{z}=1 / 3 ; \Upsilon_{x}=1 / 3 ; \Upsilon_{y}=1 / 3\right)$, with $P_{\text {eff }}^{m}=P_{\text {eff }}$ and $\eta_{e f f}^{m}=\eta_{\text {eff }}$.

In subsection 3.4, we demonstrated that the host thickness $(h)$ is a good parameter for controlling $P_{\text {eff }}$ and $\eta_{\text {eff. }}$ Thus, to assess the HMM performance, with and without gratings, we vary the host thickness in the range $1 \mathrm{~nm} \leq h \leq 100 \mathrm{~nm}$. Figure 4.13 shows $P_{\text {eff }}(\mathrm{a}, \mathrm{c}, \mathrm{e})$ and $\eta_{\text {eff }}(\mathrm{b}, \mathrm{d}, \mathrm{f})$ for HMM_opt (squares) and HMM_ch3 (circles) considering the scenarios I ( $a, b)$, II (c,d) and III (e,f). HMM_opt is optimized to decouple the high- $k$ modes radiated by the QEs. These modes are responsible for increasing the QEs Purcell factor $(P)$. Thus, this decoupling mechanism helps decreasing the $P_{\text {eff }}^{Z}$, as seen in Figure 4.13 (a). Nonetheless, it also helps increasing the power radiated to the far field, resulting in higher 
(a)

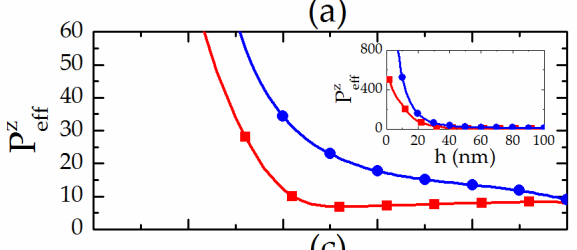

(c)

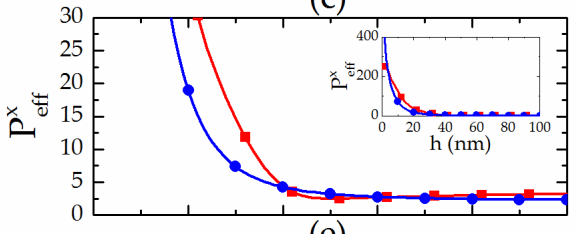

(e)

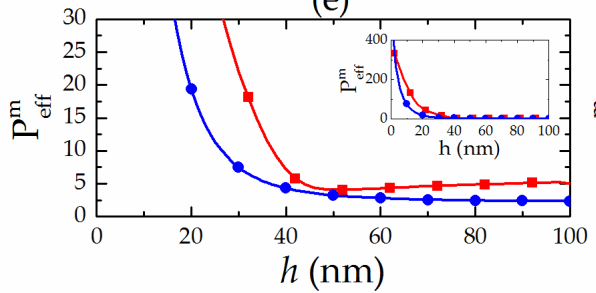

(b)

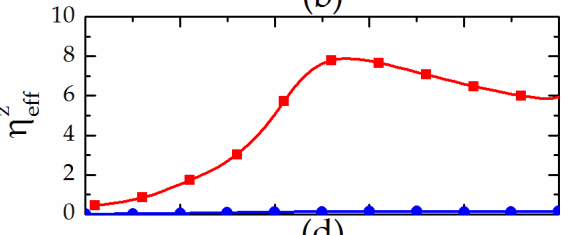

(d)

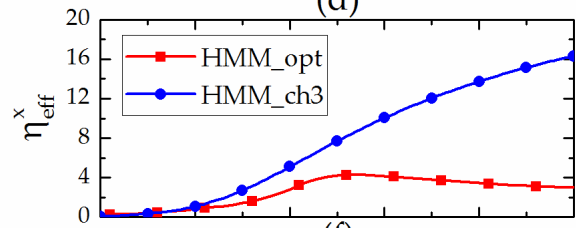

(f)

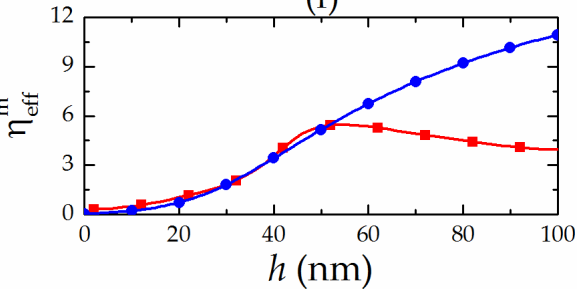

Figure 4.13. Effective Purcell factor $(\mathrm{a}, \mathrm{c}, \mathrm{e})$ and quantum efficiency $(\mathrm{b}, \mathrm{d}, \mathrm{f})$ for HMM_opt (squares) and HMM_ch3 (circles) considering scenarios I $(\mathrm{a}, \mathrm{b})$, II $(\mathrm{c}, \mathrm{d})$ and III $(\mathrm{e}, \mathrm{f}) .(\mathrm{a}, \mathrm{c}, \mathrm{e})$ are limited in the y-axis. The inset in each plot shows the full curve.

$\eta_{\text {eff }}^{z}$, as shown in Figure 4.13 (b). Notice that the $\eta_{\text {eff }}^{z}$ growth saturates at $h \approx 50 \mathrm{~nm}$, then slowly decreases. As the QEs get farther away from the HMM_opt surface, $P_{z}\left(\mathbf{r}_{0}\right)$ and $Q_{z}\left(\mathbf{r}_{0}\right)$ decrease due to the reduced power of the evanescent waves. Moreover, as seen in Figure 4.8, $P_{z}\left(\mathbf{r}_{0}\right)$ decreases faster than $Q_{z}\left(\mathbf{r}_{0}\right)$, resulting in the enhancement of $\eta_{z}\left(\mathbf{r}_{0}\right)$, and consequently of $\eta_{\text {eff }}^{z}$, as observed in Figure 4.13 (b) for $h<50 \mathrm{~nm}$. However, $Q_{z}\left(\mathbf{r}_{0}\right)$ tends to zero for $h>50 \mathrm{~nm}$ because the high- $k$ modes are converted into radiation modes decreasing $\eta_{\text {eff }}^{z}$ as a result. This explanation also applies to the behavior of $\eta_{\text {eff }}^{x}$ for HMM_opt, shown in Figure 4.13 (d).

In scenario II, $P_{\text {eff }}^{x}$ of HMM_opt is higher than that of HMM_ch3 for $h<40 \mathrm{~nm}$, as shown in Figure 4.13 (c). For $h>40 \mathrm{~nm}$ the grating does not affect significantly $P_{e f f}^{x}$, and both HMM_opt and HMM_ch3 present similar $P_{\text {eff }}$ values. The TE waves radiated by the QEs in HMM_opt are mostly reflected on the HMM surface (due to the HMM metallic behavior for TE polarization), and propagate instead in the substrate. In contrast, the reflected TE waves of HMM_ch3 propagate in air. Consequently, $Q_{x}$ is higher for HMM_ch3 than for HMM_opt, resulting in higher $\eta_{e f f}^{x}$ for the full range of $h$, as shown in Figure 4.13 (d). As seen in Figure 4.13 (b,f), the perpendicular QEs in HMM_ch3 does have significantly contribution to $g(t)$ due to their low $\eta_{e f f}^{z}$.

Finally, scenario III considers QEs equally polarized and distributed inside the host layer, and represents the most realistic scenario. Here, the perpendicular polarized QEs in HMM_ch3 have little impact on $P_{e f f}^{m}$ because of their low $\eta_{\text {eff }}^{Z}$. Therefore, $P_{e f f}^{m}$ is approximately equal to $P_{e f f}^{x}$, as explained in section 3.3. In contrast, $\eta_{\text {eff }}^{z}>\eta_{\text {eff }}^{x}$ for 
(a)

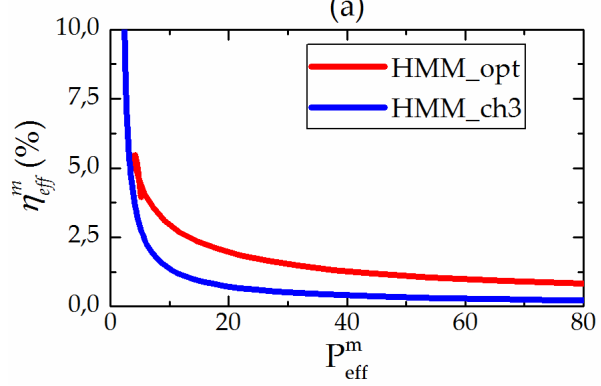

(b)

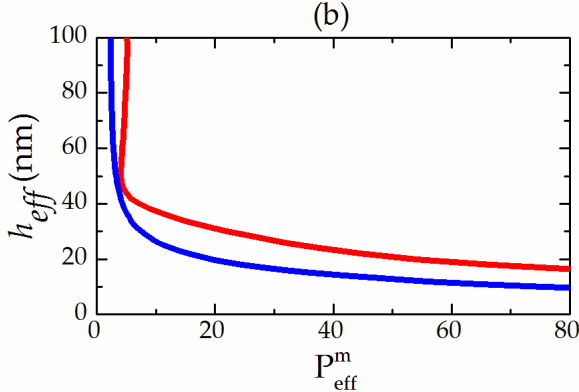

Figure 4.14. (a) and (b) show $\eta_{e f f}^{m}$ and $h_{\text {eff, }}$ respectively, as function of $P_{e f f}^{m}$ for the scenario III with HMM_opt (red lines) and HMM_ch3 (blue lines).

HMM_opt, and the perpendicularly polarized QEs strongly influence $P_{e f f}^{m}$. All these factors, along with the increased $P$ provided by perpendicularly polarized QEs, enable HMM_opt to produce high $P_{\text {eff }}^{m}$ for the entire range of $h$, as seen in Figure 4.13 (e). Note that $\eta_{e f f}^{m}$ is approximately equal for both structures for $h<50 \mathrm{~nm}$, as seen in Figure 4.13 (f) due to the strong high- $k$ mode decoupling provided by the gratings of HMM_opt that also increases $Q$. Thus, HMM_opt compensates for the reduction in $\eta_{e f f}^{m}$ caused by the enhancement of $P_{\text {eff }}^{m}$ by increasing $Q$. HMM_ch3, in contrast, shows better performance for $h>50 \mathrm{~nm}$, since $\eta_{\text {eff }}^{m}$ becomes much higher in this region.

It is also interesting to compare the performance in terms of $\eta_{e f f}^{m}$ of both HMMs as function of $P_{\text {eff, }}$ as shown in Figure 4.14 (a). Since the host thickness is proportional to the total power radiated to the far field, it is also interesting to know the value of $h$ required to achieve the desired $P_{\text {eff }}\left(h_{\text {eff }}\right)$, as shown Figure 4.14 (b). Note that HMM_opt performs better in terms of $\eta_{e f f}^{m}$ and $h_{\text {eff }}$ whenever high values of $P_{e f f}^{m}$ are required (>5) then. In contrast, if low values of $P_{e f f}^{m}$ are required, then HMM_ch3 is the appropriate choice because of its higher $\eta_{e f f}^{m}$ and $h_{\text {eff. }}$. For instance, if $P_{\text {eff }}=10$ is required for HMM_ch3, then $h$ should be equal to $28 \mathrm{~nm}$, resulting in $\eta_{\text {eff }}=1.25 \%$. To achieve the same $P_{\text {eff }}$ with HMM_opt, $h$ would have to be $38 \mathrm{~nm}$, achieving $\eta_{\text {eff }}=3.00 \%$. Therefore, besides increasing the total radiated power, our optimization approach can increase the system's quantum efficiency in $240 \%$ for the desired $P_{\text {eff. }}$.

In summary, HMM_opt allows the same $P_{\text {eff }}$ to be achieved for $h<50$ with a higher $\eta_{e f f}^{m}$ than that from HMM_ch3, which confirms the superior device performance provided by the optimization procedure proposed in this chapter.

\subsection{Computation improvement of our method}

In this section we discuss the gain in computational time that we obtain using the approach proposed in this thesis in comparison with commercial software. Due to software license (Lumerical) and the massive number of RCWA simulations required, we have used different computational resources to carry out the simulations. The 2D-FDTD 
(Lumerical) simulations for instance were carried out in a regular desktop computer (Intel Core i7-3960X processor with 64GB of RAM). The RCWA simulations, in contrast, were carried out in the high performance DaVinci cluster at Rice University, due to the number of simulations necessary to create the color maps. In any case, for comparison purposes, we provide below a description of the computational costs involved:

- Regarding the maps in Figure 4.8 (a-d): the reflection and transmission coefficients $\left(v_{\chi, \sigma}^{i}\left(k_{x}^{0}\right)\right)$ of each diffraction order $i$ are calculated using 2D-RCWA retaining 51 orders. To simulate a dipole, the parallel wavevector component of the incident plane wave $\left(k_{x}^{0}\right)$ is swept with 5001 points in the interval $0 \leq$ $\left|\frac{k_{x}}{k_{0}}\right| \leq 25$. Therefore, it takes approximately 6 minutes to calculate all terms $v_{\chi, \sigma}^{i}$. Using the obtained results, the maps are then calculated with $51 \times 51$ pixels where each pixel takes approximately 20 s to be calculated, resulting in a total computational cost of 14 hours. If the maps were calculated only with the 2DFDTD, the computational cost would have risen to approximately 27 days (15 minutes per pixel) per dipole orientation.

- Regarding Figure 4.12 (a-d): the reflection and transmission coefficients $\left(v_{\chi, \sigma}^{i, m}\left(k_{x}^{0}, k_{y}^{0}\right)\right)$ of each diffraction order $(i, m)$ are calculated using 3D-RCWA retaining $121(11 \times 11)$ orders. To simulate a dipole, the parallel wavevector components of the incident plane wave $\left(k_{x}\right.$ and $\left.k_{y}\right)$ are swept with 1001 points each in the interval $0 \leq\left|\frac{k_{x}}{k_{0}}\right|,\left|\frac{k_{y}}{k_{0}}\right| \leq 25$. Therefore, it takes approximately 6 days to calculate all terms $v_{\chi, \sigma}^{i, m}$. Using the obtained results, the maps are then calculated with $21 \times 21$ pixels in the quadrant $\Delta x, \Delta y \leq 210 \mathrm{~nm}$ where each pixel takes approximately $20 \mathrm{~min}$ to be calculated (the remaining quadrants are obtained by mirroring the results from $\Delta x, \Delta y \leq 210 \mathrm{~nm}$ with respect to the axes $\Delta x=210 \mathrm{~nm}, \Delta y=210 \mathrm{~nm}$ and $\Delta y=410 \mathrm{~nm}-\Delta y$ ). Therefore, the total computational time to obtain all maps is 12 days with our approach (Note: since we have carried out these simulations in the DaVinci cluster at Rice University, the computational cost is reduced to approximately 2 days). However, if these simulations were carried out entirely with 3D-FDTD, it would have taken approximately 441 days to obtain the maps in Figure 4.12 (one simulation a day per pixel).

In summary, mappings of Figure 4.8 (a-d) and Figure 4.12 (a-d) were performed 46 and 36.5 times faster, respectively, using the approach proposed here than it would if numerically calculated via FDTD.

\subsection{Chapter conclusion}

In this chapter, we have proposed a semi-analytical model to investigate the radiation emission profile of QEs under a nano-patterned metamaterial. In addition, the reduced computational burden of this method (compared to numerical approaches) allows the 
Purcell factor and the radiated power to be calculated in a more straightforward manner. The model was applied to a HMM composed of seven $\mathrm{Ag} / \mathrm{TiO}_{2}$ layers with a silver grating on its top. Using a 2D configuration first, we optimized the grating geometrical parameters and showed that the radiation pattern is highly directive for $\Lambda_{x}=420 \mathrm{~nm}$. Furthermore, the normalized power coupled from high- $k$ HMM modes into air was increased to 1.67 for perpendicular polarization for a fill factor $f f=70 \%$ and grating height $a=80 \mathrm{~nm}$. With such optimum parameters, we calculated $\Psi_{\alpha}^{2 D}$ an $P_{\alpha}^{2 D}$ as function of the $\mathrm{QE}$ relative position with respect to the grating groove and ridge. The maximum $\Psi_{\alpha}^{2 D}$ and $P_{\alpha}^{2 D}$ values occurred at the same QE relative position, which helped increase the system's overall Purcell factor, according to (28). After the 2D optimization procedure, we applied the optimized parameters to a 3D periodic array of nano-cylinders. We observed that the radiation pattern lost some of its directivity because of cross-polarized transmission; however, the QE showed the highest Purcell factor $\left(P_{z}=145\right.$ and $\left.P_{x}=67.5\right)$ and the highest radiated power $\left(\Psi_{z}^{3 D}=2\right.$ and $\left.\Psi_{x}^{3 D}=0.75\right)$ for $q=10 \mathrm{~nm}$. Moreover, in comparison with the HMM without the gratings, we have demonstrated that our approach is capable of enhancing the system's quantum efficiency by $240 \%$ if a Purcell factor of 10 is of interest. To the best of our knowledge, the model described in this chapter is the first in the literature capable of mapping, in a detailed and accurate fashion, both the QE s' Purcell factor and total radiated power under or above any nano-patterned structure as function of position. 



\section{Chapter 5 Semi-analytical modeling of QEs embedded in nano- patterned HMM}




\section{Chapter 5 - Summary}

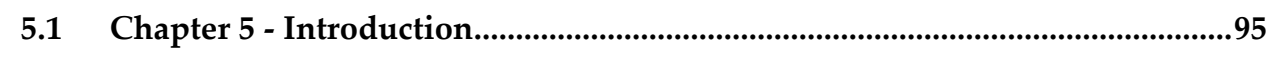

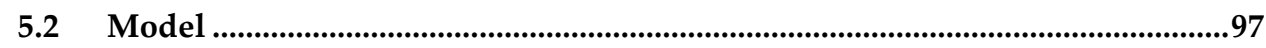

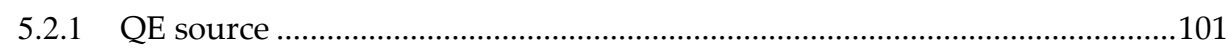

5.2.1.1 Calculating $\eta^{\mathrm{fp}}$ and $\Gamma^{\mathrm{fp}}$ for an arbitrary QE configuration .....................102

5.2.1.2 Calculating $\eta^{\mathrm{fp}}$ and $\Gamma^{\mathrm{fp}}$ for an arbitrary QE configuration ....................104

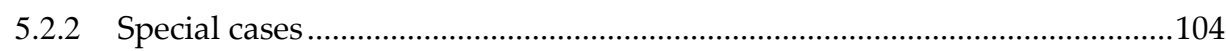

5.2.2.1 Three simultaneous sources at fixed positions (substrate, cover, and wth

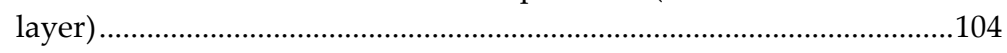

5.2.2.2 QEs uniformly distributed in the wth layer (QEL)................................105

5.2.2.3 Sources uniformly distributed in each layer ...........................................106

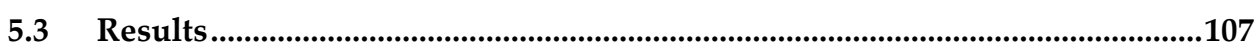

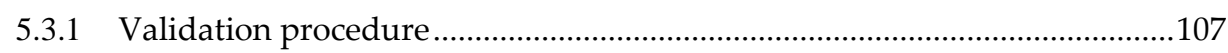

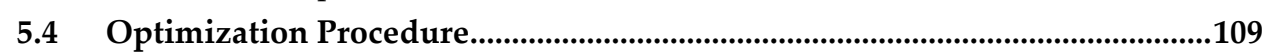

5.4.1 Step 1: 3D-SAM optimization of a non-patterned HMM..............................109

5.4.2 Step 2: NPHM period and fill-factor optimization with the 2D-SAM ........111

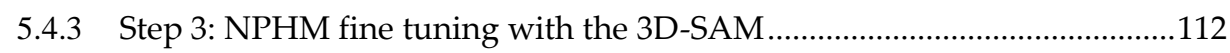

5.5 Chapter conclusion ...............................................................................................116 


\subsection{Chapter 5 - Introduction}

HMMs can be obtained from wire media [38], [94]-[96] and from planar stacks of thin alternating metal/dielectric layers [37], [38], [40], [97]-[100]. The latter is preferred because its dispersion profile can be easily tuned by changing materials, layer thicknesses, or wavelength [37]. Its large PDoS allows faster spontaneous emission rate [109], shorter lifetimes and higher QE dissipated power. Unfortunately, the spatial dispersion induced by high- $k$ modes in real HMMs limits the number of supported modes and, consequently, the PDoS [101]. In addition, most of the dissipated power coupled into the HMM is due to the high- $k$ modes which, in turn, do not couple to free-space modes [109], [124]. Consequently, the energy of these modes is mostly thermally dissipated inside the HMM, reducing the QE's $\eta$. Some authors have resorted to nano-patterned HMMs (NPHM) to convert high- $k$ modes into free-space modes $\left(k \leq k_{0}\right)$ to increase $\eta$. For instance, in [55], [103], [125]-[127], [156] the authors have used a patterned HMM surrounded by QEs, while in [128], [129] the QEs are placed inside the HMM. Metallic or dielectric gratings on the top of the HMM have also been used for this purpose, where QEs are in the layer below the HMM [124], or in, where they are inside it [57], [120], [121]. Regardless of the geometry, the optimization of the NPHM geometrical parameters is usually carried out via numerical simulations, such as finite-difference time-domain (FDTD) and finite elements (FEM) methods, which are computationally costly. An alternative to improve the optimization process is to use semi-analytical techniques, such as in [131], [132] where QEs are allowed in any layer of a non-patterned structure, or in [124] where QEs are placed close to the NPHM either in the top or bottom semi-infinite layer. Moreover, only a single (dipole) source can be used with these techniques, rendering them not general enough.

In this context, we propose here a general semi-analytical model to calculate, both in two and three dimensions (2D and 3D), all radiation parameters of any electromagnetic source type (including QEs modeled as a dipole) embedded in any of the layers of a nanopatterned stratified media (NSM). This method allows multiple electromagnetic sources arbitrarily distributed in the cover, substrate, and any inner layer to be used simultaneously. The flexibility of placing the source inside any layer (nano-patterned or not) allows us to very efficiently obtain the radiation emission parameters of any previously published $\mathrm{HMM} /$ source configuration [2,20-34]. The validation of the proposed method is carried out by calculating $\Gamma$ and $Q$ of a two-source system consisting of a $\mathrm{QE}$ embedded in a medium bounded by nano-patterned layers ( $\mathrm{QEL}_{\mathrm{L}}$ ) and a dipole source in the cover layer $\left(\mathrm{QEC}_{\mathrm{c}}\right)$. We also investigate the influence of $\mathrm{QEL}_{\mathrm{L}}$ on $\mathrm{QEc}$ 's emission parameters and compare the results with FDTD simulations.

Once validated, we apply the proposed model to a more realistic situation where a NPHM is designed to enhance both the $\Gamma$ and $\eta$ of a QE. These parameters are mapped here as function of the QE and NPHM relative position, which is facilitated by the method's low computational cost. This mapping is a helpful tool to understand the decay behavior of the whole system [55], [114] since QEs are randomly distributed and oriented inside the NPHM [55], [57], [103], [120], [121], [125]-[129]. By knowing the QE's $\Gamma$ and $\eta$ at each 
position allows the decay curve $g(t)$ of a system with multiple QEs to be analytically calculated. This procedure is fundamentally different from those currently used in the literature, where the decay curve is either fitted as a sum of exponentials [121], [126], [130] or calculated from $\Gamma$ and $\eta$ at each position [55], [114]. These approaches resort to computationally costly numerical simulations to calculate $\Gamma$ and $\eta$. Our procedure, in contrast, is not only more accurate but also computationally more attractive and can be applied to any NSM. Furthermore, the analytically calculated $g(t)$ allows the system's effective quantum efficiency $\eta_{\text {eff }}$ and emission rate $\Gamma_{\text {eff }}$ to be directly obtained assuming multiple arbitrarily distributed electromagnetic sources in the cover, substrate, and any inner layer. In this sense, we also propose here a new procedure to optimize the NPHM geometrical parameters to maximize $\eta_{\text {eff }}$ while achieving the desired $\Gamma_{\text {eff. }}$. The optimization procedure is divided into three steps, as follows: Step 1) The 3D semi-analytical model (3D-SAM) is used to optimize the layer thicknesses of a non-patterned HMM to maximize $\eta_{\text {eff. }}$ We take advantage of the structure's axial symmetry and lack of nano-patterns to reduce the 3D-SAM computational cost even further and thus calculate the $\Gamma^{f p}$ and $\eta^{f p}$ maps (required to calculate $\eta_{\text {eff }}$ and $\Gamma_{\text {eff }}$ ), where the superscript $f p$ denotes a source at a fixed position. Step 2) The computationally efficient 2D semi-analytical model (2D-SAM) is employed to optimize the nano-pattern's period and fill-factor. This helps hasten the final optimization step based on the more realistic 3D model. Step 3) The 2D optimized parameters are fed into the 3D model for final tuning. In this step, the full $\Gamma^{f p}$ and $\eta^{f p}$ maps are built for a few different scenarios to evaluate the ones capable of achieving the desired $\Gamma_{\text {eff. }}$ The proposed procedure is then applied to maximize the $\eta_{\text {eff }}$ of a system whose host (polymer) layer is embedded with Rhodamine 6G (R6G), a fluorescent molecule, while increasing $\Gamma_{\text {eff }}$ by a factor of 10 (actually, it can be any value). We show that a NPHM consisting of 9 alternating layers of silver $(\mathrm{Ag})$ and silicon dioxide $\left(\mathrm{SiO}_{2}\right)$ can be optimized to enhance $\eta_{\text {eff }}$ from $4.3 \%$ (non-patterned) up to $7.3 \%$ and $11.6 \%$ if patterned along one (1D-NPHM) and two axes (2D-NPHM), respectively. We also show that the computational time required to build the $\Gamma^{f p}$ and $\eta^{f p}$ maps, used in the calculation of $g(t)$, is reduced by $96 \%$ (1D-NPHMs) and $98 \%$ (2D-NPHMs) when compared to FDTD simulations.

This chapter is organized as follows: Section 5.2 presents the mathematical model used to calculate $\eta_{\text {eff }}$ and $\Gamma_{\text {eff, }}$ both in 2D and 3D. Section 5.3 begins with the validation procedure which consists in calculating $\eta$ and $Q$ of a two-source double nano-patterned structure and comparing the results with those of FDTD simulations. With the validation procedure complete, we then follow steps 1 and 2 of the optimization procedure to find the best NPHM layer thicknesses, period and fill-factor to maximize $\eta_{\text {eff. }}$. Finally, we carry out step 3 to map $\Gamma^{f p}$ and $\eta^{f p}$ for all possible QE positions for a 1D- and 2D-NPHM using the 3D-SAM. These maps are then utilized to calculate $\eta_{\text {eff }}$ and $\Gamma_{\text {eff }}$ for different source scenarios. Section 5.5 presents some concluding remarks. Part of the work presented in this chapter has been submitted as:

A. F. Mota, A. Martins, V. Pepino, H. Ottevaere, W. Meulebroeck, F. L. Teixeira, and B.H. V. Borges, "Semi-analytical modeling of quantum emitters embedded in nanopatterned hyperbolic metamaterials," submitted to Journal of the Optical Society of America B. 


\subsection{Model}

The proposed model assumes a $W$-layer stack sandwiched between two semi-infinite slabs, i.e., a substrate with permittivity $\varepsilon s$ and a cover layer with permittivity $\varepsilon c$, as shown in Figure 5.1. The permittivity of the $w$ th $(w \in[1 . . W])$ layer is $\varepsilon_{w}$, with periodic cell size $\Lambda_{x}^{w}$ and $\Lambda_{y}^{w}$ along the $x$ - and $y$-directions, respectively. Note that the period of each nanopatterned layer may be different (non-proportional), so it is convenient to define $\Lambda_{x}$ and $\Lambda_{y}$ relative to a supercell, given by $\Lambda_{x}=\operatorname{LCM}\left\{\Lambda_{x}^{1} . . \Lambda_{x}^{W}\right\}$ and $\Lambda_{y}=\operatorname{LCM}\left\{\Lambda_{y}^{1} . . \Lambda_{y}^{W}\right\}$, where LCM stands for the least common multiple. Differently from [124], the proposed model allows for up to three sources to be embedded into the system, one in layer $w$ (source L), one in the substrate (source $S$ ) and one in the cover layer (source $C$ ). Assuming that the sources S, L, C are located at $z=z_{s}, z_{L}$ and $z_{c}$, respectively, their 2D Fourier transformed (FT) electric fields can be obtained as follows [124]:

$\mathbf{E}_{\Delta}\left(\mathbf{k}_{\|}, z\right)=\left\{\begin{array}{l}\mathbf{E}_{\Delta}^{+}\left(\mathbf{k}_{\|}, z\right)=\left[E_{\Delta}^{p,+}\left(\mathbf{k}_{\|}\right) \mathbf{P}_{\mathbf{p}}^{\Delta}\left(\mathbf{k}_{\|}\right)+E_{\Delta}^{s,+}\left(\mathbf{k}_{\|}\right) \mathbf{P}_{\mathbf{s}}^{\Delta}\left(\mathbf{k}_{\|}\right)\right] e^{j k_{2 \Delta}\left(z-z_{\Delta}\right)},\left(z-z_{\Delta}\right)>0 \\ \mathbf{E}_{\Delta}^{-}\left(\mathbf{k}_{\|}, z\right)=\left[E_{\Delta}^{p,-}\left(\mathbf{k}_{\|}\right) \mathbf{P}_{\mathbf{p}}^{\Delta}\left(\mathbf{k}_{\|}\right)+E_{\Delta}^{s,-}\left(\mathbf{k}_{\|}\right) \mathbf{P}_{\mathbf{s}}^{\Delta}\left(\mathbf{k}_{\|}\right)\right] e^{-j k_{2 \Delta}\left(z-z_{\Delta}\right)},\left(z-z_{\Delta}\right) \leq 0\end{array}\right.$,

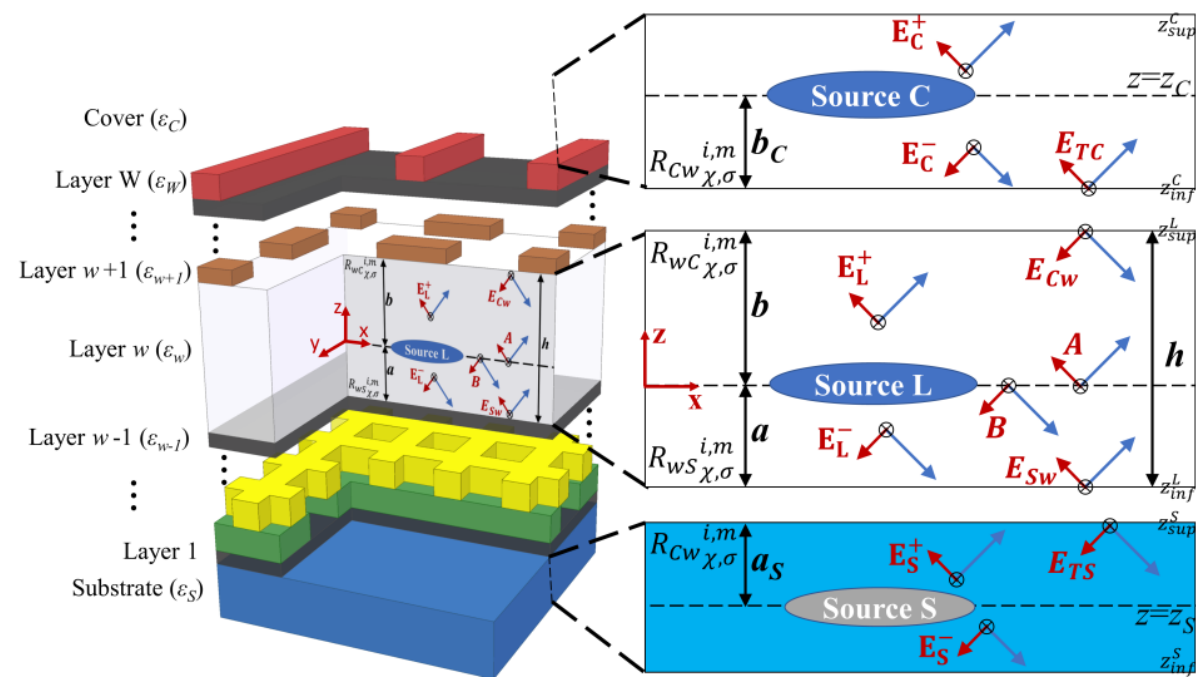

Figure 5.1. W-layer stack sandwiched between two semi-infinite slabs, i.e., a substrate and a cover layer. The depicted nano-pattern profiles are just an illustration of possible geometries. $\Lambda_{x}^{w}$ and $\Lambda_{y}^{w}$ represent the $w$ th layer unit cell sizes along $\mathrm{x}-$ and $\mathrm{y}$ directions, respectively. The sources $S, L$ and $C$ are embedded within the substrate, $w$ th layer and cover, respectively. The electric fields radiated by the sources $S\left(\mathbf{E}_{\mathbf{S}}^{+}\right.$ and $\left.\mathbf{E}_{\mathbf{S}}^{-}\right)$and $C\left(\mathbf{E}_{\mathbf{C}}^{+}\right.$and $\left.\mathbf{E}_{\mathbf{C}}^{-}\right)$are transmitted to layer $w$ giving rise to the fields $\mathbf{E}_{\mathbf{S w}}$ and $\mathbf{E}_{\mathbf{C} w}$, respectively. These fields, combined with the electric field radiated by the source $\mathrm{L}\left(\mathbf{E}_{\mathbf{L}}^{+}\right.$and $\left.\mathbf{E}_{\mathbf{L}}^{-}\right)$, are reflected by layers $w+1$ and $w-1$, creating two stationary fields (A and $\mathbf{B}$ ) inside layer $w$. Moreover, the sum of the electric fields propagating along the $+\mathbf{z}$ and $-\mathbf{z}$ directions inside layer $w$ are transmitted to the cover $\left(\mathbf{E}_{\mathbf{T C}}\right)$ and substrate $\left(\mathbf{E}_{\mathbf{T S}}\right)$, respectively. 
where $\mathbf{E}_{\Delta}^{+}$and $\mathbf{E}_{\Delta}^{-}$are the electric field vectors radiated by source $\Delta$ ( $\Delta=S, \mathrm{~L}$ or $\mathrm{M}$ ) along $+\mathbf{z}-$ and -z directions, respectively, $E_{\Delta}^{\chi,+}$ and $E_{\Delta}^{\chi,+}$ are electric field amplitudes with $\chi$ polarization ( $\chi=s$ or $p$ for transversal electric (TE) and magnetic (TM) polarizations, respectively) propagating along $\mathbf{z}$ and $-\mathbf{z}$ directions, respectively. $\mathbf{k}_{\|}=k_{x} \mathbf{x}+k_{y} \mathbf{y}$ is the parallel component of the wavevector $\mathbf{k}_{\Delta}=\mathbf{k}_{\|}+k_{z \Delta} \mathbf{z}$, where $k_{\Delta}=\left|\mathbf{k}_{\Delta}\right|=2 \pi \sqrt{\epsilon_{\Delta}} / \lambda_{0}$ $\left(\varepsilon_{L}=\varepsilon_{w}\right)$, and $\varepsilon_{\Delta}$ is the permittivity of the layer where the source $\Delta$ is located ( $\varepsilon_{L}=\varepsilon_{w}, \varepsilon_{c}=\varepsilon_{c}$ and $\varepsilon s=\varepsilon_{s}$ ) and $\lambda_{0}$ is the wavelength in vacuum. Finally, $\mathbf{P}_{\chi}^{\Delta}$ is the vector used to decompose the electric field into the $x-, y$ - and $z$-components inside the layer in which the source $\Delta$ is located. Assuming temporal dependence $e^{-j \omega t}, \mathbf{P}_{\chi}^{\Delta}$ is written as [124]:

$$
\begin{gathered}
\mathbf{P}_{\mathbf{s}}^{\Delta}\left(\mathbf{k}_{\mid ।}\right)=\left[\begin{array}{lll}
\frac{k_{y}}{\sqrt{k_{x}^{2}+k_{y}^{2}}} & -\frac{k_{x}}{\sqrt{k_{x}^{2}+k_{y}^{2}}} & 0
\end{array}\right]^{\mathrm{T}}, \\
\mathbf{P}_{\mathbf{p}}^{\Delta}\left(\mathbf{k}_{\mid ।}\right)=\left[\frac{k_{x} k_{z \Delta}}{k_{\Delta} \sqrt{k_{x}^{2}+k_{y}^{2}}} \frac{k_{y} k_{z \Delta}}{k_{\Delta} \sqrt{k_{x}^{2}+k_{y}^{2}}}-\frac{\xi}{k_{\Delta}}\right]^{\mathrm{k}} .
\end{gathered}
$$

As seen in Figure 5.1, $\mathbf{E}_{\mathbf{S}}^{+}$and $\mathbf{E}_{\mathbf{C}}^{-}$are transmitted to layer $w$ generating the electric field vectors $\mathbf{E}_{\bar{\Delta} \mathbf{w}}=\left[E_{\bar{\Delta} \mathbf{w}}^{p} \mathbf{P}_{\mathbf{p}}^{\mathbf{L}}+E_{\bar{\Delta} \mathbf{w}}^{s} \mathbf{P}_{\mathbf{s}}^{\mathbf{L}}\right] e^{j k_{z L}\left|z-z_{0}\right|}$, where $z_{0}=-a$ or $b$ for $\bar{\Delta}=S$ or $\mathrm{C}$, respectively. Furthermore, $\mathbf{E}_{\mathbf{L}}^{+}, \mathbf{E}_{\mathbf{L}}^{-}, \mathbf{E}_{\mathbf{S w}}$ and $\mathbf{E}_{\mathbf{C w}}$ are reflected at the upper and bottom interfaces creating two stationary fields, one propagating along $\mathbf{z}\left(\mathbf{A}=\left[A^{p} \mathbf{P}_{\mathbf{p}}^{\mathbf{L}}+A^{s} \mathbf{P}_{\mathbf{s}}^{\mathbf{L}}\right] e^{\mathbf{j}\left(k_{z L}\right) z}\right)$ and the other propagating along $-\mathbf{z}\left(\mathbf{B}=\left[B^{p} \mathbf{P}_{\mathbf{p}}^{\mathbf{L}}+B^{s} \mathbf{P}_{\mathbf{s}}^{\mathbf{L}}\right] e^{-\mathrm{j}\left(k_{z L}\right) z}\right)$. Note also that the blue and red arrows in Figure 5.1 represent the wavevector and the electric field, respectively. If the nano-patterning is not present, then the fields for each $\mathbf{k}_{\|}$point can be solved individually [109]. However, the nano-patterning diffracts the incident light (with moment $\left.\mathbf{k}_{\|}^{\mathbf{0}}\right)$ into the $i$ th and $m$ th orders $\left(\mathbf{k}_{\|}^{\mathbf{0}}+i K_{x} \mathbf{x}+\mathrm{m} K_{y} \mathbf{y}\right)$ in the $k_{x}$ and $k_{y}$ directions, respectively, creating a coupling mechanism between the harmonics. Consequently, all harmonics need to be solved at once. Hence, we propose to write the amplitude of the 2D Fourier transformed electric field $\Psi^{\chi}\left(\Psi^{\chi}=A^{\chi}, B^{\chi}, E_{\Delta}^{\chi, \pm}\right.$ or $\left.E_{\bar{\Delta} w}^{\chi}\right)$ as a modulated comb function $\left(\Psi_{\text {comb }}^{\chi}\right)$ with period equal to the grating supercell moment $K_{x}\left(K_{x}=2 \pi / \Lambda_{x}\right)$ and $K_{y}\left(K_{y}=2 \pi / \Lambda_{y}\right)$ along the $k_{x}$ and $k_{y}$ axes, respectively, and centered at $\mathbf{k}_{\|}^{0}=k_{x}^{0} \mathbf{x}+k_{y}^{0} \mathbf{y}$, as follows

$$
\begin{gathered}
\psi_{\text {comb }}^{\chi}\left(\mathbf{k}_{\|}^{0}, z\right)=\sum_{i, m} \psi^{\chi}\left(\mathbf{k}_{\|}, z\right) \delta\left(k_{x}-k_{x}^{0}-i K_{x}\right) \delta\left(k_{y}-k_{y}^{0}-m K_{y}\right) \\
=\sum_{i, m} \psi_{i, m}^{\chi}\left(\mathbf{k}_{\|}^{0}, z\right) \delta\left(k_{x}-k_{x}^{0}-i K_{x}\right) \delta\left(k_{y}-k_{y}^{0}-m K_{y}\right)
\end{gathered}\left\{\begin{array}{l}
0 \leq k_{x}^{0}<K_{x} \\
0 \leq k_{y}^{0}<K_{y}{ }^{\prime}
\end{array}\right.
$$

where $\Psi_{i, m}^{\chi}\left(\mathbf{k}_{\|}^{\mathbf{0}}, z\right)$ is the $i$ th and $m$ th element in the $k_{x}$ and $k_{y}$ directions of the comb function, respectively, with $k_{x}^{0}$ and $k_{y}^{0}$ limited to the interval $0 \leq\left(k_{x}^{0}, k_{y}^{0}\right) \leq\left(K_{x}, K_{y}\right)$ so that no multiple identical combs exists. After solving the $\Psi_{c o m b}^{\chi}$ for each point of the proposed interval, the total electric field $\Psi \times$ is recalculated as 


$$
\psi^{\chi}\left(\mathbf{k}_{\|}, z\right)=\int_{0}^{K_{x}} \int_{0}^{K_{y}} \sum_{i, m} \psi_{i, m}^{\chi}\left(\mathbf{k}_{\|}^{0}, z\right) \delta\left(k_{x}-k_{x}^{0}-i K_{x}\right) \delta\left(k_{y}-k_{y}^{0}-m K_{y}\right) \mathrm{d} k_{x}^{0} \mathrm{~d} k_{y^{\prime}}^{0}
$$

where $\delta($.$) is the Dirac delta function. For ease of notation, we define the i$ th and $m$ th harmonics of $\mathbf{k}_{\|}^{\mathbf{0}}\left(\mathbf{k}_{\|}^{\mathrm{i}, \mathbf{m}}\right)$ and $k_{z \Delta}\left(k_{z \Delta}^{i, m}\right)$ in the $k_{x}$ and $k_{y}$ directions, respectively, as follows,

$$
\begin{gathered}
\mathbf{k}_{\|}^{\mathrm{i}, \mathrm{m}}\left(\mathbf{k}_{\|}^{0}\right)=k_{x}^{i} \mathbf{x}+k_{y}^{m} \mathbf{y}=\left(k_{x}^{0}-i K_{x}\right) \mathbf{x}+\left(k_{y}^{0}-m K_{y}\right) \mathbf{y}, \\
k_{z \Delta}^{i, m}=\sqrt{k_{\Delta}^{2}-\left(k_{x}^{i}\right)^{2}-\left(k_{y}^{m}\right)^{2}} .
\end{gathered}
$$

After defining the electric field as comb functions, $E_{\bar{\Delta} w, i, m}^{\chi}$ can be calculated as follows:

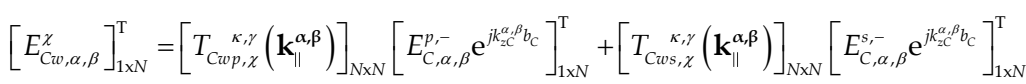

$$
\begin{aligned}
& {\left[E_{S w, \alpha, \beta}^{\chi}\right]_{1 \times N}^{\mathrm{T}}=\left[T_{S w p, \chi}{ }^{k, \gamma}\left(\mathbf{k}_{\|}^{\alpha, \beta}\right)\right]_{N \times N}\left[E_{S, \alpha, \beta}^{p,+} \mathrm{e}^{j k_{2 s}^{\alpha, \beta} a_{S}}\right]_{1 \times N}^{\mathrm{T}}+\left[T_{S w s, \chi}^{\kappa_{s, \gamma}}\left(\mathbf{k}_{\|}^{\alpha, \beta}\right)\right]_{N \times N}\left[E_{S, \alpha, \beta}^{s,+} \mathrm{e}^{j \mathrm{j}_{s, \beta}^{\alpha, \beta} a_{S}}\right]_{1 \times N}^{\mathrm{T}}}
\end{aligned}
$$

where [ $]_{\text {QxT }}$ represents a matrix with $Q$ lines and $T$ columns. The indices $q$ and $t$ represent the matrix's line and row, respectively, $T_{C w_{\chi, \sigma}}{ }_{\chi, \gamma}$ and $T_{S w_{\chi, \sigma}}{ }_{\chi, \gamma}$ are the transmission coefficients (from cover and substrate to the $w$ th layer, respectively) of the $\kappa$ th and $\gamma$ th diffraction orders along $x$ and y directions. The subscripts $\chi$ and $\sigma$ indicate the polarization of the incident and transmitted waves, respectively. Differently from 2D systems (nano-pattern along one axis), 3D systems (nano-pattern along two axes) give rise to cross-polarized

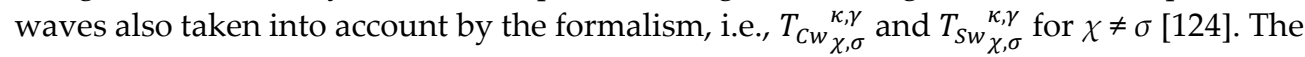
transmission coefficients are calculated with the semi-analytical rigorous coupled wave analysis method (RCWA) [152]-[154] for a total number of diffraction orders $N=N_{x} N_{y}$, with $N_{x}$ and $N_{y}$ as the number of diffraction orders along the $x$ and $y$ axes, respectively. The auxiliary variables used in (5.8) and (5.9) $(\alpha, \beta, \kappa$ and $\gamma)$ are calculated as follows:

$$
\left\{\begin{array}{c}
\alpha=\text { floor }\left(\frac{t-1}{N_{y}}\right)-\frac{\left(N_{x}-1\right)}{2} \\
\beta=t-\frac{\left(N_{y}-1\right)}{2}-\text { floor }\left(\frac{t-1}{N_{y}}\right) N_{y} \\
\kappa=\alpha-\text { floor }\left(\frac{q-1}{N_{y}}\right)+\frac{\left(N_{x}-1\right)}{2} \\
\gamma=\beta-\left[q-1+\frac{\left(N_{y}-1\right)}{2}-\left(\alpha-\kappa+\frac{\left(N_{x}-1\right)}{2}\right) N_{y}\right]
\end{array} .\right.
$$

The stationary fields A and B are obtained with the help of the 2D Fourier transformed electric fields after applying the boundary conditions at $z=-a$ and $z=b$. This results in the following linear system: 


\section{$\mathbf{F}=\mathbf{G H}$}

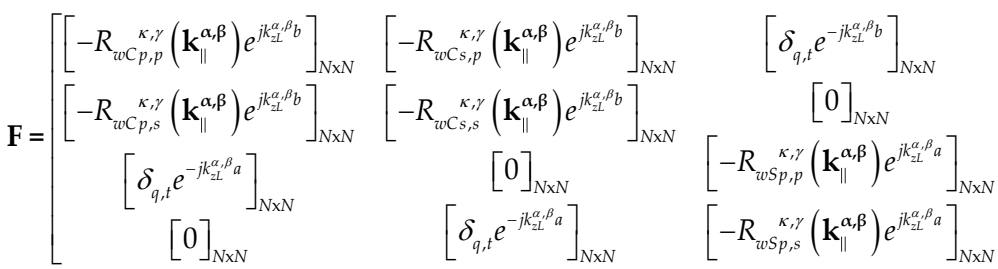

$$
\begin{aligned}
& \left.\begin{array}{c}
{[0]_{N \times N}} \\
{\left[\delta_{q, t} e^{-j k_{L L}^{\alpha, \beta} b}\right]_{N \times N}} \\
{\left[-R_{w w p, s}^{\kappa, \gamma}\left(\mathbf{k}_{\|}^{\alpha, \beta}\right) e^{j k_{2 L}^{\alpha, \beta} a}\right]_{N \times N}} \\
{\left[-R_{w S S, s}^{\kappa, \gamma}\left(\mathbf{k}_{\|}^{\alpha, \beta}\right) e^{j k_{L L}^{\alpha, \beta} a}\right]_{N \times N}}
\end{array}\right], \\
& \mathbf{G}=\left[\begin{array}{l}
{\left[A_{\alpha, \beta}^{p}\right]_{1 \times N}^{\mathrm{T}}} \\
{\left[A_{\alpha, \beta}^{s}\right]_{1 \times N}^{\mathrm{T}}} \\
{\left[B_{\alpha, \beta}^{p}\right]_{1 \times N}^{\mathrm{T}}} \\
{\left[B_{\alpha, \beta}^{s}\right]_{1 \times N}^{\mathrm{T}}}
\end{array}\right]
\end{aligned}
$$

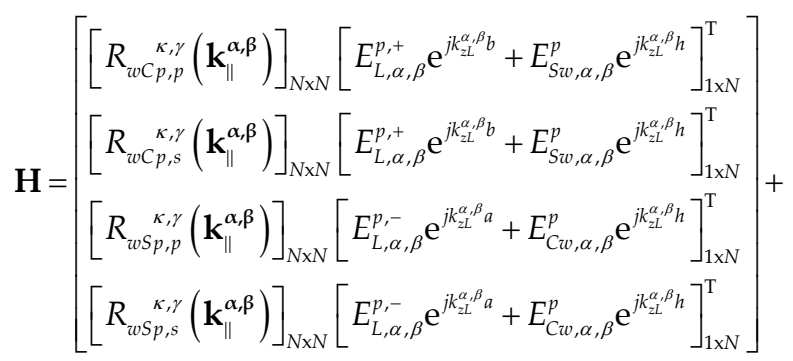

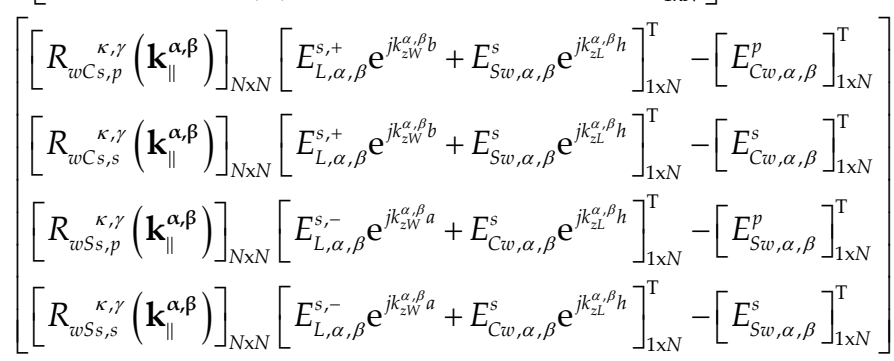

where $\delta_{q, t}$ is the Kronecker delta, $R_{w C_{\chi, \sigma}}^{\kappa, \gamma}$ and $R_{w s_{\chi, \sigma}}^{\kappa, \gamma}$ are the reflection coefficients (from the $w$ th layer to the cover and to the substrate, respectively) of the $\kappa$ th and $\gamma$ th diffraction orders along $x$ and y directions. The reflection coefficients are calculated with RCWA [152]-[154] for a total number of diffraction orders $N=N_{x} N_{y}$. The accuracy of the proposed method depends on the number of diffracted orders $N$. However, a large $N$ also increases the computational cost required to solve the linear system (5.11). After calculating the 
stationary fields A and B from (5.11), the 2D FT of the electric fields transmitted to the cover (Етс) and to the substrate (ETs) are calculated as follows:

$$
\begin{aligned}
& \mathbf{E}_{\mathrm{T}}\left(\mathbf{k}_{\|}, z\right)=\left[E_{T \bar{\Delta}}^{p}\left(\mathbf{k}_{\|}\right) \mathbf{P}_{\mathbf{p}}^{\bar{\Delta}}\left(\mathbf{k}_{\|}\right)+E_{T \bar{\Delta}}^{s}\left(\mathbf{k}_{\|}\right) \mathbf{P}_{s}^{\bar{\Delta}}\left(\mathbf{k}_{\|}\right)\right] \mathrm{e}^{j k_{z \bar{\Delta}}\left|z-z_{\bar{\Delta}}\right|}, \\
& E_{T C}^{\chi}\left(\mathbf{k}_{\|}\right)=\sum_{i, m=-N_{x}, N_{y}}^{N_{x}, N_{y}}\left\{\left[E_{L}^{p,+}\left(\mathbf{k}_{\|}^{\mathbf{i}, \mathbf{m}}, b\right)+A^{p}\left(\mathbf{k}_{\|}^{\mathrm{i}, \mathbf{m}}, b\right)+E_{S w}^{p}\left(\mathbf{k}_{\|}^{\mathrm{i}, \mathbf{m}}, b\right)\right] T_{w c c^{\prime}, \chi}^{i, m}\left(\mathbf{k}_{\|}^{\mathrm{i}, \mathbf{m}}\right)+\right. \\
& {\left[E_{L}^{s,+}\left(\mathbf{k}_{\|}^{\mathbf{i}, \mathbf{m}}, b\right)+A^{s}\left(\mathbf{k}_{\|}^{\mathbf{i}, \mathbf{m}}, b\right)+E_{S w}^{s}\left(\mathbf{k}_{\|}^{\mathbf{i}, \mathbf{m}}, b\right)\right] T_{w C S, \chi}{ }^{i, m}\left(\mathbf{k}_{\|}^{\mathbf{i}, \mathbf{m}}\right)+} \\
& \left.E_{C}^{p,-}\left(\mathbf{k}_{\|}^{\mathrm{i}, \mathrm{m}}, z_{C}-b_{c}\right) R_{C w p, \chi}^{i, m}\left(\mathbf{k}_{\|}^{\mathrm{i}, \mathrm{m}}\right)+E_{C}^{s,-}\left(\mathbf{k}_{\|}^{\mathrm{i}, \mathrm{m}}, z_{C}-b_{c}\right) R_{\mathrm{C} w s, \chi}^{i, m}\left(\mathbf{k}_{\|}^{\mathrm{i}, \mathbf{m}}\right)\right\}, \\
& E_{T S}^{\chi}\left(\mathbf{k}_{\|}\right)=\sum_{i, m=-N_{x}, N_{y}}^{N_{x}, N_{y}}\left\{\left[E_{L}^{p,-}\left(\mathbf{k}_{\|}^{\mathbf{i}, \mathbf{m}}, a\right)+B^{p}\left(\mathbf{k}_{\|}^{\mathbf{i}, \mathbf{m}}, a\right)+E_{C w}^{p}\left(\mathbf{k}_{\|}^{\mathbf{i}, \mathbf{m}}, a\right)\right] T_{w s p, \chi}^{i, m}\left(\mathbf{k}_{\|}^{\mathbf{i}, \mathbf{m}}\right)+\right. \\
& {\left[E_{L}^{s,-}\left(\mathbf{k}_{\|}^{\mathbf{i}, \mathbf{m}}, b\right)+B^{s}\left(\mathbf{k}_{\|}^{\mathbf{i}, \mathbf{m}}, a\right)+E_{C w}^{s}\left(\mathbf{k}_{\|}^{\mathbf{i}, \mathbf{m}}, \mathbf{a}\right)\right] T_{w S s, \chi}^{i, m}\left(\mathbf{k}_{\|}^{\mathbf{i}, \mathbf{m}}\right)+} \\
& \left.E_{S}^{p,+}\left(\mathbf{k}_{\|}^{\mathbf{i}, \mathbf{m}}, z_{S}-a_{S}\right) R_{S w p, \chi}^{i, m}\left(\mathbf{k}_{\|}^{\mathbf{i}, \mathbf{m}}\right)+E_{S}^{s,+}\left(\mathbf{k}_{\|}^{\mathbf{i}, \mathbf{m}}, z_{S}-a_{S}\right) R_{S w s, \chi}^{i, m}\left(\mathbf{k}_{\|}^{\mathbf{i}, \mathbf{m}}\right)\right\},
\end{aligned}
$$

where $R_{C w_{\chi, \sigma}}^{i, m}$ and $T_{w C}{ }^{i, m}, \sigma$ are the reflection (from the cover to the $w$ th layer) and transmission (from the $w$ th layer to the cover) coefficients, respectively, of the $i$ th and $m$ th diffraction orders along the $x$ and y directions; $R_{S w_{\chi, \sigma}}^{i, m}$ and $T_{w s_{\chi, \sigma}}^{i, m}$ are the reflection (from the substrate to the $w$ th layer) and transmission (from the wth layer to the substrate) coefficients respectively, of the $i$ th and $m$ th diffraction orders along the $x$ and y directions. After using the stationary phase method to propagate the FT electric field into the far field, the electric field transmitted to the cover $\left(\overline{\mathbf{E}_{\mathrm{TC}}}\right)$ or to the substrate $\left(\overline{\mathbf{E}_{\mathrm{TS}}}\right)$ is obtained as [109]:

$$
\begin{aligned}
& \overline{\mathbf{E}_{\mathbf{T} \bar{\Delta}}}=-\mathrm{j} k_{z \bar{\Delta}} \frac{\mathrm{e}^{\mathrm{j} k_{\bar{z} \bar{\Delta}} r}}{r}\left\{\left[E_{T \bar{\Delta}}^{p}\left(k_{\bar{\Delta}} \sin \theta \cos \varphi, k_{\bar{\Delta}} \sin \theta \sin \varphi\right) \boldsymbol{\theta}+\right.\right. \\
&\left.\left.E_{T \bar{\Delta}}^{s}\left(k_{\bar{\Delta}} \sin \theta \cos \varphi, k_{\bar{\Delta}} \sin \theta \sin \varphi\right) \varphi\right]\right\} .
\end{aligned}
$$

Finally, the total power radiated by all sources in the system (L, S, C located at positions $\mathrm{Z}=\mathbf{r} \mathbf{L}, \mathbf{r s}, \mathbf{r c}$, respectively) towards the cover $\left(Q_{c}\right)$ or the substrate $\left(Q_{s}\right)$ is calculated as follows:

$$
Q_{\bar{\Delta}}=\frac{1}{2 I_{\bar{\Delta}}} \int_{0}^{\pi / 2} \int_{0}^{2 \pi}\left|\overline{\mathbf{E}_{\mathrm{T} \bar{\Delta}}}\right|^{2} r^{2} \sin \theta \mathrm{d} \varphi \mathrm{d} \theta,
$$

here $I_{\bar{\Delta}}=\sqrt{\mu_{\bar{\Delta}} / \epsilon_{\bar{\Delta}}}$ is the cover's $(\bar{\Delta}=\mathrm{C})$ or substrate's $(\bar{\Delta}=\mathrm{S})$ impedance. The formalism used to calculate $\Gamma$ and $\eta$ assuming the sources as QEs is described in the next section.

\subsubsection{QE source}

Here, each $\mathrm{QE}_{\Delta}$ source is modeled as a dipole source with momentum $\mathbf{p}_{\Delta}$. In most applications, the QEs are randomly distributed and oriented inside a layer (either c, s or 
w) and each QE affects the system's total decay rate. Therefore, to evaluate the decay curve $g(t)$ and effective quantum efficiency $\eta_{\text {eff }}$ of such a system, we need first to calculate $\eta^{f p}$ and $\Gamma^{f p}$ at all possible positions $(\mathbf{r s}, \mathbf{r} \mathbf{L}, \mathbf{r c})$ and orientations $\left(\mathbf{p}_{\Delta}=p_{\Delta} \mathbf{X}, p_{\Delta} \mathbf{y}\right.$ or $\left.p_{\Delta} \mathbf{z}\right)$, weighted by the probability of that position being occupied by a $Q E\left(\Gamma^{f p} \equiv \Gamma_{f p}\left(\mathbf{r} \mathbf{c}, \mathbf{r} \mathbf{L}, \mathbf{r s}, \mathbf{p} \mathrm{c}, \mathbf{p}_{\mathbf{L}}, \mathbf{p s}\right)\right.$ and $\left.\eta^{f p} \equiv \eta f^{f p}\left(\mathbf{r c}, \mathbf{r} \mathbf{L}, \mathbf{r s}, \mathbf{p c}_{\mathbf{c}} \mathbf{p} \mathbf{L}, \mathbf{p s}\right)\right)$.

\subsubsection{Calculating $\eta^{f p}$ and $\Gamma^{f p}$ for an arbitrary $Q E$ configuration}

The first step is to write the 2D FT electric field radiated by $\mathrm{QE}_{\Delta}$ placed at an arbitrary position $\mathbf{r}_{\Delta}=x_{\Delta} \mathbf{x}+y_{\Delta} \mathbf{y}+z_{\Delta} \mathbf{z}$ as a comb function, as in (5.4), where the $i$ th and $m$ th harmonics in the $k_{x}$ and $k_{y}$ directions, respectively, are written as [124]:

$$
\begin{aligned}
& E_{\Delta, i, m}^{p, \pm}\left(\mathbf{k}_{\|}^{\mathrm{i}, \mathrm{m}}, z\right)=\mathrm{j} \frac{\omega^{2} \mu_{\Delta}}{4 \pi} \mathrm{e}^{ \pm j\left[\mathbf{k}_{z \Delta}^{i, m}\left(z-z_{\Delta}\right)+k_{x}^{i} x_{\Delta}+k_{y}^{m} y_{\Delta}\right]}\left[\begin{array}{c}
\frac{k_{x}^{i}}{k_{\Delta} \sqrt{\left(k_{x}^{i}\right)^{2}+\left(k_{y}^{m}\right)^{2}}} \\
\frac{k_{y}^{m}}{k_{\Delta} \sqrt{\left(k_{x}^{i}\right)^{2}+\left(k_{y}^{m}\right)^{2}}} \\
\mp \frac{\sqrt{\left(k_{x}^{i}\right)^{2}+\left(k_{y}^{m}\right)^{2}}}{k_{\Delta} \mathbf{k}_{z \Delta}^{i, m}}
\end{array}\right] \cdot \mathbf{p}_{\Delta}, \\
& E_{\Delta, i, m}^{s, \pm}\left(\mathbf{k}_{\|}^{\mathbf{i}, \mathbf{m}}, z\right)=\mathrm{j} \frac{\omega^{2} \mu_{\Delta}}{4 \pi} \mathrm{e}^{ \pm i\left[\mathbf{k}_{z \Delta}^{i, m}\left(z-z_{\Delta}\right)+k_{x}^{i} x_{\Delta}+k_{y}^{m} y_{\Delta}\right]}\left[\begin{array}{c}
\frac{k_{y}^{m}}{\mathbf{k}_{z \Delta}^{i, m} \sqrt{\left(k_{x}^{i}\right)^{2}+\left(k_{y}^{m}\right)^{2}}} \\
\frac{k_{x}^{i}}{\mathrm{k}_{z \Delta}^{i, m} \sqrt{\left(k_{x}^{i}\right)^{2}+\left(k_{y}^{m}\right)^{2}}} \\
0
\end{array}\right] \cdot \mathbf{p}_{\Delta},
\end{aligned}
$$

where $\omega$ is the angular frequency and $\mu_{\Delta}$ is the magnetic permeability of the medium in which the source $\Delta$ is located. The stationary fields $\mathbf{A}$ and $\mathbf{B}$ are found by substituting (5.20) and (5.21) into (5.11) and then solving the resulting linear system. The spontaneous emission rate of the source $\Delta\left(\Gamma_{\Delta}\right)$ with internal quantum efficiency equals to 1 is calculated as:

$$
\begin{aligned}
& \frac{\Gamma_{\Delta}\left(\mathbf{r}_{\Delta}\right)}{\Gamma_{0}}=\frac{\omega}{2 W_{0}} \operatorname{Im}\left\{\mathbf{p}_{\Delta}^{*} \cdot\left[\mathbf{A}^{\mathrm{IF}}\left(\mathbf{r}_{\Delta}\right)+\mathbf{B}^{\mathrm{IF}}\left(\mathbf{r}_{\Delta}\right)+\mathbf{E}_{\mathrm{L}}^{+, \mathrm{IF}}\left(\mathbf{r}_{\Delta}\right)+\mathbf{E}_{\mathrm{SW}}^{\mathrm{IF}}\left(\mathbf{r}_{\Delta}\right)+\mathbf{E}_{\mathrm{Cw}}^{\mathrm{IF}}\left(\mathbf{r}_{\Delta}\right)\right]\right\}, \text { if } \Delta=\mathrm{L} \\
& \frac{\Gamma_{\Delta}\left(\mathbf{r}_{\Delta}\right)}{\Gamma_{0}}=\frac{\omega}{2 W_{0}} \operatorname{Im}\left\{\mathbf{p}_{\Delta} * \cdot\left[\mathbf{E}_{\mathrm{T} \Delta}^{\mathrm{IF}}\left(\mathbf{r}_{\Delta}\right)+\mathbf{E}_{\Delta}^{+, \mathrm{IF}}\left(\mathbf{r}_{\Delta}\right)\right]\right\}, \text { if } \Delta=\mathrm{C} \text { or S }
\end{aligned}
$$


where $\Gamma_{0}$ is the QE's emission rate in vacuum, $W_{0}$ is the power emitted by a dipole in free space $\left(W_{0}=p^{2} \omega k_{0}^{2} / 12 \pi \epsilon_{0}\right)$, and $\Psi^{\mathrm{IF}}\left(\Psi=\mathbf{A}, \mathbf{B}, \mathbf{E}_{\mathbf{L}}^{+}, \mathbf{E}_{\mathbf{S W}}, \mathbf{E}_{\mathbf{C W}}, \mathbf{E}_{\mathbf{S}}^{ \pm}, \mathbf{E}_{\mathbf{C}}^{ \pm}, \mathbf{E}_{\mathrm{TS}}\right.$ or $\left.\mathbf{E}_{\mathrm{TC}}\right)$ is the electric field calculated using the $2 \mathrm{D}$ inverse FT as follows,

$$
\Psi^{\mathrm{IF}}(\mathbf{r})=\frac{1}{2 \pi} \int_{-\infty}^{\infty} \int \boldsymbol{\Psi}\left(\mathbf{k}_{\|}, z\right) \mathrm{e}^{\mathrm{j}\left(k_{x} x+k_{y} y\right)} \mathrm{d} k_{x} \mathrm{~d} k_{y} .
$$

The calculation of $\eta$ requires the knowledge of the total power dissipated by the dipole and the portion radiated towards the far field region of the substrate and cover. Thus, we must evaluate the electric field in the far field to calculate the former. From (18), the temporal envelop of the electric field transmitted to the cover $\left(\overline{\mathbf{E}_{\mathbf{T C}}}\right)$ or to the substrate $\left(\overline{\mathbf{E}_{\mathbf{T S}}}\right)$ in the far field region is calculated as [109]

$$
\begin{aligned}
\overline{\mathbf{E}_{\mathrm{T} \bar{\Delta}}}(t)=-\mathrm{j} k_{z \bar{\Delta}} \frac{\mathrm{e}^{\mathrm{j} k_{\overline{\bar{\Delta}}} r}}{r} \sum_{\Delta=S, L, C}\left\{\left[E_{T \bar{\Delta}}^{p, \Delta}\left(k_{\bar{\Delta}} \sin \theta \cos \varphi, k_{\bar{\Delta}} \sin \theta \sin \varphi\right) \theta+\right.\right. \\
\left.\left.E_{T \bar{\Delta}}^{s, \Delta}\left(k_{\bar{\Delta}} \sin \theta \cos \varphi, k_{\bar{\Delta}} \sin \theta \sin \varphi\right) \varphi\right] \mathrm{e}^{-\Gamma_{\Delta} t}\right\},
\end{aligned}
$$

where $\theta$ and $\phi$ are the elevation and azimuthal angles, $E_{T \bar{\Delta}}^{\chi, \Delta}$ is the amplitude of the 2D FT electric field transmitted from the source $\Delta$ to the cover $(\bar{\Delta}=\mathrm{C})$ or to the substrate $(\bar{\Delta}=\mathrm{S})$. $E_{T \bar{\Delta}}^{\chi, \Delta}$ is calculated with (5.16) and (5.17) when only the source $\Delta$ is embedded in the system. The total power radiated by all the sources in the system $(\mathrm{L}, \mathrm{S}, \mathrm{C}$ located at positions $\mathrm{z}=$ $\mathbf{r}_{\mathrm{L}}, \mathbf{r s}, \mathbf{r c}$, respectively) towards the cover $\left(Q_{c}\right)$ or the substrate $\left(Q_{s}\right)$ is calculated as follows

$$
Q_{\bar{\Delta}}(t)=\frac{1}{2 I_{\bar{\Delta}}} \int_{0}^{\pi / 2} \int_{0}^{2 \pi}\left|\overline{\mathbf{E}_{\mathrm{T} \bar{\Delta}}}(t)\right|^{2} r^{2} \sin \theta \mathrm{d} \varphi \mathrm{d} \theta .
$$

The total dissipated power by the three QEs is given by

$$
W_{t}=W_{0} \Gamma^{f p}
$$

where $\Gamma^{f p}$ is the normalized power dissipated by the three QEs defined as,

$$
\Gamma^{f p}=\sum_{\Delta=S, L, C} \frac{\Gamma_{\Delta}}{\Gamma_{0}} .
$$

Finally, the system's quantum efficiency $\left(\eta^{f p}\right)$ with QEL, QEc and QEs at fixed positions z $=\mathbf{r} \mathbf{L}, \mathbf{r s}$ and $\mathbf{r c}$, respectively, is given by the ratio between their total radiated power at $t=$ 0 and their total dissipated power, as follows:

$$
\eta^{f p}=[100 \%] \times \frac{Q_{S}(0)+Q_{C}(0)}{W_{0} \Gamma^{f p}} .
$$

Therefore, the mapping of $\eta^{f p}$ and $\Gamma^{f p}$ for all possible positions ( $\left.\mathbf{r s}, \mathbf{r}_{\mathrm{L}}, \mathbf{r c}\right)$ and polarizations $\mathbf{p}=\left(\mathbf{p}_{\Delta}=p_{\Delta \mathbf{X}}, p_{\Delta} \mathbf{y}\right.$ or $\left.p_{\Delta \mathbf{z}}\right)$ is accomplished by repeating this procedure for the desired values of $\mathbf{r}_{\Delta}$ and $\mathbf{p}_{\Delta}$ in (5.20) and (5.21) and solving (5.27) and (5.28) for each case. 


\subsubsection{Calculating $\eta^{f p}$ and $\Gamma^{f p}$ for an arbitrary $Q E$ configuration}

The $\eta^{f p}$ and $\Gamma^{f p}$ mappings are now used to estimate the system's effective response due to a random distribution of sources inside each layer. Thus, the system's effective quantum efficiency $\eta_{\text {eff }}$ is calculated by averaging the efficiency of each QE in all possible configurations as follows,

$$
\eta_{e f f}=\int_{V_{S}} \int_{V_{L}} \int_{V_{C}} \sum_{\mathbf{p}_{\mathbf{C}}, \mathbf{p}_{\mathbf{L}}, \mathbf{p}_{\mathbf{S}}} F\left(\mathbf{r}_{\mathrm{C}}, \mathbf{r}_{\mathbf{L}}, \mathbf{r}_{\mathrm{S}}, \mathbf{p}_{\mathrm{C}}, \mathbf{p}_{\mathbf{L}}, \mathbf{p}_{\mathbf{S}}\right) \eta^{f p} \mathrm{~d} V_{C} \mathbf{d} V_{L} \mathrm{~d} V_{S}
$$

where $V_{\Delta}$ is the volume where each $\mathrm{QE}_{\Delta}$ is found $\left(\left[-\Lambda_{x} / 2,-\Lambda_{y} / 2, z_{\text {inf }}^{\Delta}\right] \leq[x, y, z]<\left[\Lambda_{x} / 2, \Lambda_{y} / 2\right.\right.$, $\left.\left.z_{\text {sup }}^{\Delta}\right]\right), z_{\text {inf }}^{\Delta}$ and $z_{\text {sup }}^{\Delta}$ are the lower and upper limits in $z$-axis where the source $\Delta$ can be placed. $F$ is defined as the probability density function that describes the probability of a given position being occupied by a randomly positioned and oriented QE. Similarly, the normalized time-dependent decay curve $g_{\bar{\Delta}}(t)$ measured in the cover $(\bar{\Delta}=C)$ or the substrate $(\bar{\Delta}=\mathrm{S})$ is calculated as follows [114],

$$
g_{\bar{\Delta}}(t)=\frac{1}{\eta_{e f f} W_{0}} \iint_{V_{S}} \int_{V_{L}} \sum_{V_{C}} \sum_{\forall \mathbf{p}_{\mathrm{C}}, \mathbf{p}_{\mathbf{L}}, \mathbf{p}_{\mathrm{S}}} F\left(\mathbf{r}_{\mathrm{C}}, \mathbf{r}_{\mathbf{L}}, \mathbf{r}_{\mathrm{S}^{\prime}}, \mathbf{p}_{\mathrm{C}^{\prime}}, \mathbf{p}_{\mathbf{L}^{\prime}}, \mathbf{p}_{\mathrm{S}}\right)\left[\frac{Q_{\bar{\Delta}}(t)}{\Gamma^{f p}}\right] \mathrm{d} V_{C} \mathrm{~d} V_{L} \mathrm{~d} V_{S}
$$

In (5.30), the normalized time-dependent exponential decay $\left(g_{\bar{\Delta}}(t)\right)$ is weighted by the QE's quantum efficiency at each position. After calculating $g_{\bar{\Delta}}(t)$, the system's effective lifetime $\left(\tau_{\text {eff }}\right)$ is obtained through the condition $g_{\bar{\Delta}}\left(\tau_{\text {eff }}\right)=1 / \mathrm{e}$, resulting in the effective enhancement of spontaneous emission rate $P=\Gamma_{\text {eff }} / \Gamma_{0}=\tau_{0} / \tau_{\text {eff }}$, where $\tau_{0}$ is the QE's lifetime in freespace.

\subsubsection{Special cases}

In this section, we reduce the formalism to the case where the QEs are uniformly distributed and oriented inside each layer. This assumption greatly simplifies the calculation of $\eta_{\text {eff }}$ and $g_{\bar{\Delta}}$, and is normally adopted when polymers embedded with QEs are spin-coated on top of the structure [57], [103], [120], [121], [124]-[128]. Thus, we evaluate (5.29) and (5.30) for the following scenarios:

I. three fixed QEs, one in the wth layer, one in the substrate and one in the cover layer. This scenario is used to validate the proposed method;

II. sources uniformly distributed and oriented in the NPHM's wth layer.

III. sources uniformly distributed and oriented in the cover, the wth layer and substrate.

\subsubsection{Three simultaneous sources at fixed positions (substrate, cover, and wth layer)}

In this case, $\mathrm{QE}_{\Delta}$ is fixed at the position $\mathbf{r}_{\Delta}$ with polarization $\mathbf{p}_{\Delta}$. Thus, $F=\delta\left(\mathbf{r c}, \mathbf{r w}, \mathbf{r s}, \mathbf{p} c_{\text {, }}\right.$ pw, ps) and (5.29) and (5.30) are rewritten as: 


$$
\begin{gathered}
\eta_{e f f}=\eta^{f p}=[100 \%] \times \frac{Q_{S}(0)+Q_{C}(0)}{W_{0} \sum_{\Delta=S, L, C} \frac{\Gamma_{\Delta}}{\Gamma_{0}}}, \\
g_{\bar{\Delta}}(t)=\left[\frac{Q_{\bar{\Delta}}(t)}{\Gamma^{f p}}\right]=\frac{Q_{\bar{\Delta}}(t)}{W_{0} \sum_{\Delta=S, L, C} \frac{\Gamma_{\Delta}}{\Gamma_{0}}} .
\end{gathered}
$$

Notice that (5.31) and (5.32) require $Q_{\bar{\Delta}}, \eta^{f p}, \Gamma_{\Delta}$ to be calculated at a single position and polarization. The method validation is carried out by comparing $Q_{\bar{\Delta}}, \Gamma_{\Delta}$ with those directly obtained with the FDTD simulation.

\subsubsection{QEs uniformly distributed in the wth layer $\left(Q E_{L}\right)$}

Here we assume that only the $w$ th layer is embedded with QEs (QEL). Therefore, the triple integral in (5.29) and (5.30) is reduced to a single integral over $V_{L}$ (defined as the region [$\left.\left.\Lambda_{x} / 2,-\Lambda_{y} / 2,-\mathrm{a}\right] \leq[x, y, z]<\left[-\Lambda_{x} / 2,-\Lambda_{y} / 2, b\right]\right)$. is reduced to a single integral over $V_{L}$ (defined as the region $\left.\left[-\Lambda_{x} / 2,-\Lambda_{y} / 2,-\mathrm{a}\right] \leq[x, y, z]<\left[\Lambda_{x} / 2, \Lambda_{y} / 2, b\right]\right)$. Furthermore, $F$ becomes dependent only of $\mathbf{r}_{\mathrm{L}}$ and $\mathrm{p}_{\mathrm{L}}$ and can be written as follows,

$$
F\left(\mathbf{r}_{\mathbf{L}}, \mathbf{p}_{\mathbf{L}}\right)=\frac{1}{3 \Lambda_{x} \Lambda_{y} h}, \mathbf{r}_{\mathbf{L}} \in V_{L}
$$

The summation in (5.29) and (5.30) is carried out only for the three possible QEL orientations $\left(\mathbf{p}_{\mathbf{L}}=p_{L} \mathbf{X}, p_{L} \mathbf{y}\right.$ or $\left.p_{L \mathbf{Z}}\right)$. The following definitions are adopted to relax the notation,

$$
\left\{\begin{array}{l}
Q_{\Delta}^{\alpha}=\left.Q_{\bar{\Delta}}\right|_{\mathbf{p}_{\mathbf{W}}=p_{W} \alpha} \\
\Gamma_{\alpha}=\left.\Gamma^{f p}\right|_{\mathbf{p}_{\mathbf{W}}=p_{W} \alpha} \\
\eta_{\alpha}=\left.\eta^{f p}\right|_{\mathbf{p}_{W}=p_{W} \alpha}
\end{array} .\right.
$$

With these assumptions, $\eta_{\text {eff }}$ and $g_{\bar{\Delta}}(t)$ are given by,

$$
\begin{gathered}
\eta_{e f f}=\frac{1}{3 \Lambda_{x} \Lambda_{y} h} \int_{-\frac{\Lambda_{y}}{2}-\frac{\Lambda_{x}}{2}}^{\frac{\Lambda_{y}}{2}} \int_{-a}^{\frac{\Lambda_{x}}{2}} \sum_{\alpha=x, y, z} \eta_{\alpha} \mathrm{d} z \mathrm{~d} x \mathrm{~d} y . \\
g_{\bar{\Delta}}(t)=\frac{1}{3 \Lambda_{x} \Lambda_{y} h \eta_{e f f} W_{0}} \int_{-\frac{\Lambda_{y}}{2}}^{\frac{\Lambda_{y}}{2}} \int_{-\frac{\Lambda_{x}}{2}}^{\frac{\Lambda_{x}}{2}} \int_{-a}^{b} \sum_{\alpha=x, y, z} \frac{Q_{\Delta}^{\alpha}(t)}{\Gamma_{\alpha} / \Gamma_{0}} \mathrm{~d} z \mathrm{~d} x \mathrm{~d} y,
\end{gathered}
$$


SEMI-ANALYTICAL MODELING OF QES EMBEDDED IN NANO-PATTERNED HMM

\subsubsection{Sources uniformly distributed in each layer}

Finally, in the case where the QEs are uniformly distributed and oriented inside each layer $(\mathrm{w}, \mathrm{S}, \mathrm{C})$, the probability density function is given by

$$
F\left(\mathbf{r}_{\mathrm{C}}, \mathbf{r}_{\mathrm{L}}, \mathbf{r}_{\mathrm{S}}, \mathbf{p}_{\mathrm{C}}, \mathbf{p}_{\mathrm{L}}, \mathbf{p}_{\mathrm{S}}\right)=\frac{1}{27 \Omega_{C} \Omega_{L} \Omega_{S}}, \mathbf{r}_{\Delta} \in V_{\Delta} .
$$

where $\Omega_{\Delta}$ is the volume of region $V_{\Delta}$. Furthermore, the triple integral over $V_{S}, V_{L}$ and $V_{C}$ are performed for all possible polarization combinations $(3 \times 3 \times 3=27$ possibilities). With these assumptions, $\eta_{\text {eff }}$ and $g_{\bar{\Delta}}(t)$ are calculated as follows,

$$
\begin{gathered}
\eta_{e f f}=\frac{1}{27 \Omega_{C} \Omega_{L} \Omega_{S}} \int_{V_{S}} \int_{V_{L}} \int_{V_{C}} \sum_{\forall \mathbf{p}_{\mathrm{C}}, \mathbf{p}_{\mathrm{L}}, \mathbf{p}_{\mathrm{S}}} \eta^{f p} \mathrm{~d} V_{C} \mathrm{~d} V_{L} \mathrm{~d} V_{S} \\
g_{\bar{\Delta}}(t)=\frac{1}{27 \Omega_{C} \Omega_{L} \Omega_{S} \eta_{e f f} W_{0}} \int_{V_{S}} \int_{V_{L}} \int_{V_{C}} \sum_{\forall \mathbf{p}_{\mathrm{C}}, \mathbf{p}_{\mathrm{L}}, \mathbf{p}_{\mathrm{S}}}\left[\frac{Q_{\bar{\Delta}}(t)}{\Gamma^{f p}}\right] \mathrm{d} V_{C} \mathrm{~d} V_{L} \mathrm{~d} V_{S}
\end{gathered}
$$




\subsection{Results}

We devide this section into two parts. In the first part, a validation procedure is carried out between the proposed method (3D-SAM) and Lumerical FDTD [151] (used here as benchmark) for a double nano-patterned 11-layer stack excited by two distinct electromagnetic sources. In the second part, we stress our method by carrying out an optimization procedure aiming at maximizing $\eta_{\text {eff }}$ for a desired value of $P$ for two nanopatterned geometries (1D- and 2D-NPHM) with the host (PMMA) layer embedded with R6G.

\subsubsection{Validation procedure}

The multilayer stack adopted in this procedure consists of $W=11$ layers built on top of a $\mathrm{SiO}_{2}$ substrate, as illustrated in Figure 5.2. Layers 1-9 consist of alternating $\mathrm{Ag}$ (with $\varepsilon=-$ $12+0.37 \mathrm{j})$ and $\mathrm{SiO}_{2}$ (with $\varepsilon=2.132$ ) layers whose thicknesses are $d_{m e}$ and $d_{d i}(15$ and $60 \mathrm{~nm})$, respectively, except for layer 9 whose thickness is $d_{m e} / 2$ as suggested in [101]. The nanopattern periodicity is $\Lambda_{x}^{s}=\Lambda_{y}^{s}=230 \mathrm{~nm}$, groove width $\rho_{g}^{s}=50 \mathrm{~nm}$ and 3D fill-factor $f_{3 D}=f_{2 D}^{2}=\left(\rho_{g}^{\mathrm{s}} / \Lambda_{x, y}^{\mathrm{s}}\right)^{2}=0.6125$, filled with polymethylmethacrylate (PMMA). Layer 11 consists of a nano-patterned layer of $\mathrm{TiO}_{2}(\varepsilon=5.95)$ with thickness $d_{c}=50 \mathrm{~nm}$, period $\Lambda_{x}^{c}=$ $\Lambda_{y}^{c}=115 \mathrm{~nm}$, groove width $\rho_{g}^{c}=57.5 \mathrm{~nm}\left(f_{3 D}=0.25\right)$, filled with air (which is also the cover
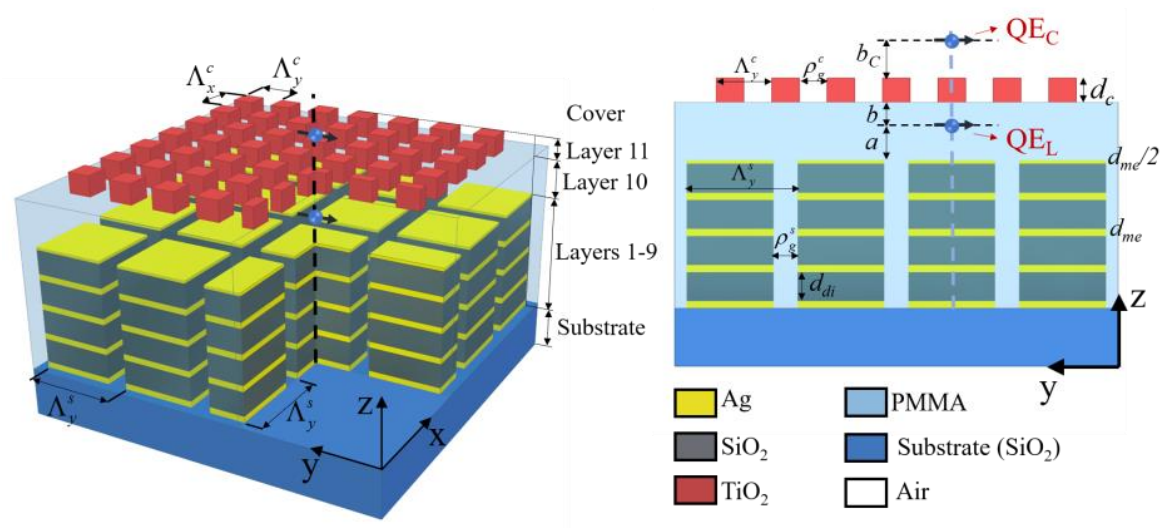

Figure 5.2. Multilayer stack with $W=11$ built on top of a $\mathrm{SiO}_{2}$ substrate. Layers 1-9 consist of alternating $\mathrm{Ag}$ and $\mathrm{SiO}_{2}$ layers whose thicknesses are $d_{m e}$ and $d_{d i}(15$ and $60 \mathrm{~nm}$ ), respectively, except for layer 9 , whose thickness is $d_{m e} / 2$. These layers are patterned with period $\Lambda_{x}^{s}=\Lambda_{y}^{s}=230 \mathrm{~nm}$, fill factor $f_{3 D}=0.6125\left(\rho_{g}^{c}=50 \mathrm{~nm}\right)$, filled with polymethylmethacrylate (PMMA). Layer 11 consists of a nano-patterned layer of $\mathrm{TiO}_{2}$ with thickness $d_{c}=50 \mathrm{~nm}$, period $\Lambda_{x}^{c}=\Lambda_{y}^{c}=115 \mathrm{~nm}, f_{3 D}=0.25\left(\rho_{g}^{c}=57.5 \mathrm{~nm}\right)$, filled with air (which is also the cover layer). The source QEL is placed inside layer 10 (PMMA) at $a=30 \mathrm{~nm}$ from layer 9 top interface and $b=20 \mathrm{~nm}$ from layer 11 bottom interface. The source QEc is placed in the cover layer at a distance $b_{c}$ from layer 11 top interface. 
layer). To stress the method, we assume two QE sources embedded in the system. The first source (QEL) is placed inside layer 10 (PMMA host layer) at $a=30 \mathrm{~nm}$ from layer 9 top interface and $b=20 \mathrm{~nm}$ from layer 11 bottom interface. The second source is placed inside the cover layer (QEc) at a distance $b_{c}$ from layer 11 top interface. Both sources are oriented parallel to the interfaces and the emission wavelength $\left(\lambda_{0}\right)$ is $548 \mathrm{~nm}$, which is the peak emission of a R6G [157]. Since both the stack and layer 11 have different nanopattern periods, we need to define a supercell period along the $x$ and $y$ axes, i.e., $\Lambda_{x}=\Lambda_{y}=$ $230 \mathrm{~nm}$. To investigate the influence of QEL on the radiation emission parameters of QEc, we need to calculate the spontaneous emission enhancement of both sources for scenarios with and without QEL, using both the 3D-SAM and Lumerical FDTD [87].

Figure 5.3 (a) shows $\Gamma_{L} / \Gamma_{0}$ (red, circles) and $\Gamma_{C} / \Gamma_{0}$ (blue, squares) obtained with 3D-SAM (solid lines) and FDTD (symbols). This figure also shows $\Gamma_{C} / \Gamma_{0}$ obtained with 3D-SAM when QEL is not present (dashed line) for comparison sake. The 3D-SAM results show good agreement with those of FDTD, confirming the accuracy of our model. The small distance between QEL and the HMM surface $(30 \mathrm{~nm})$ contributes to the increase of $\Gamma_{\mathrm{L}} / \Gamma_{0}$ (up to 15) due to the high coupling between the evanescent modes with the HMM high- $k$ modes [124]. Moreover, the power radiated by QEc that reaches QEL is smaller than that dissipated by the high- $k$ modes, making the influence of QEc on $\Gamma_{L} / \Gamma_{0}$ very small and independent of $b_{c}$, as seen Figure 5.3 (a). In contrast, QEv strongly influences $\Gamma_{c} / \Gamma_{0}$ because its radiation emission, especially the evanescent waves, constructively interferes with that from $\mathrm{QEC}_{\mathrm{c}}$ therefore enhancing its dissipated power and, consequently, $\Gamma_{\mathrm{C}} / \Gamma_{0}$. Furthermore, as $b_{c}$ increases, the evanescent waves decay exponentially, reducing the influence of QEv on $\Gamma_{\mathrm{C}} / \Gamma_{0}$, as indicated by the $\Gamma_{\mathrm{C}} / \Gamma_{0}$ convergence for the scenarios with and without QEL.

Another important aspect is the power radiated to the far field in the cover layer $\left(Q_{c}\right)$. Figure 5.3 (b) shows $Q_{c} / W_{0}$ calculated as function of $b_{c}$ with (solid line) and without (dashed line) QEL, and the FDTD results (squares) for the sake of comparison. Once again, the results obtained with the 3D-SAM nicely agree with those of the FDTD method. Note the increase of $Q_{c} / W_{0}$ due to the presence of the additional electromagnetic source QEL.

(a)

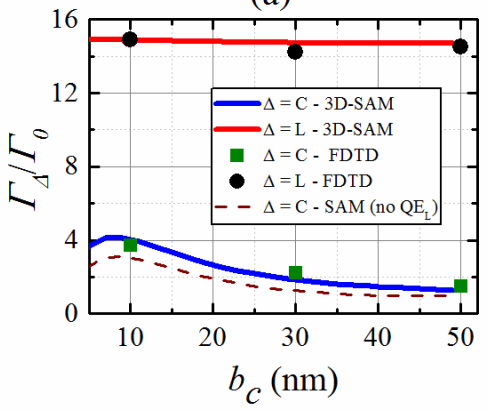

(b)

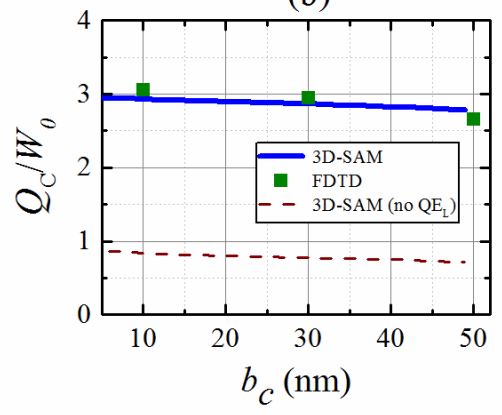

Figure 5.3. (a) $\Gamma_{L} / \Gamma_{0}$ and $\Gamma_{\mathcal{C}} / \Gamma_{0}$ using 3D-SAM (red and blue solid lines) and FDTD (symbols). This figure also shows $\Gamma_{\mathrm{C}} / \Gamma_{0}$ obtained with 3D-SAM when QEL is not present (dashed lines). (b) Qc/Wo with (solid line) and without (dashed line) QEL. 
Furthermore, as $b_{c}$ increases, $Q_{c} / W_{0}$ decreases for both scenarios, since less evanescent waves are being converted into radiated waves by the $\mathrm{TiO}_{2}$ nano-patterning. In summary, in addition to validating the proposed modeling, these results show that it is possible to control the radiation parameters of $\mathrm{QE}_{\mathrm{C}}\left(\Gamma_{\mathrm{C}} / \Gamma_{0}\right.$ and $\left.Q_{\mathcal{C}} / W_{0}\right)$ with the addition of a second electromagnetic source (QEL).

\subsection{Optimization Procedure}

Now we apply the proposed model to a more realistic case, with R6G (assumed as a uniformly distributed source, $\mathrm{QEL}_{\mathrm{L}}$ ) embedded in a PMMA host layer ( $\varepsilon=2.22$, thickness $h$ ) on top of a NPHM. As mentioned, the proposed optimization procedure maximizes $\eta_{\text {eff }}$ for any desired $P$. However, in this example, we set $P=10$ because it represents a good compromise between $P$ and $\eta_{\text {eff }}$ (note that according to (5.35) - (5.36), $P$ and $\eta_{\text {eff }}$ are inversely proportional). The wavelength is again $548 \mathrm{~nm}$. The optimization procedure is divided into three steps, as follows: In step (1) we optimize the layer thicknesses of a nonpatterned HMM with the 3D-SAM, taking advantage of the structure's axial symmetry and lack of nano-patterns to reduce the computation cost required to calculate $\eta_{\text {eff }}$ and $\Gamma_{\text {eff. }}$. In step (2), we use the computationally efficient 2D-SAM to optimize the nanopattern's period and fill-factor. This step helps hasten the optimization procedure in the more realistic 3D model. In step (3), we use the $2 \mathrm{D}$ optimized parameters into the 3D model for final tuning. In this step, the full $\Gamma_{\alpha}$ and $\eta_{\alpha}$ maps are built for a few different scenarios to evaluate the ones capable of achieving the desired $\Gamma_{\text {eff. }}$ Regarding the fine-tuning, we calculate $P$ and $\eta_{\text {eff }}$ for different values of $h$ to determine the one that results in $P=10$ with maximum $\eta_{\text {eff. }}$

\subsubsection{Step 1: 3D-SAM optimization of a non-patterned HMM}

Initially, we assume a non-patterned $\mathrm{HMM}$ with 9 alternating layers of $\mathrm{Ag}$ and $\mathrm{SiO}_{2}$ [101] because this arrangement causes most of the radiated power $\left(k \leq k_{0}\right)$ to be reflected back from the HMM's surface into the cover layer. The $\mathrm{Ag}$ and $\mathrm{SiO}_{2}$ layer thicknesses are $d_{m e}$ and $d_{d i}$, respectively, except for the first layer whose thickness is $d_{m e} / 2$, as depicted in Figure 5.4 (a).

The optimization procedure starts by adjusting $d_{d i}, d_{m e}$ and the host thickness $h$ to maximize $\eta_{\text {eff }}$ while maintaining $P=10$. We assume the unit cell size is infinite $\left(K_{x}, K_{y} \rightarrow\right.$ $0)$, there is no cross-polarized terms, and only the 0 th diffraction order is present $\left(N_{x}=N_{y}=N=1\right)$. With this assumption, the linear system (5.11) is split into two $2 \times 2$ systems, one for TM and another for TE, and each $\mathbf{k}_{\|}$point is decoupled and solved individually, resulting in the following set of equations:

$$
A^{\chi}\left(\mathbf{k}_{\|}\right)=\frac{E_{L}^{\chi,+}\left(\mathbf{k}_{\|}\right)+E_{L}^{\chi,-}\left(\mathbf{k}_{\|}\right) R_{w S, \chi, \chi}^{0,0}\left(\mathbf{k}_{\|}\right) \mathrm{e}^{2 j k_{z L} a}}{1-R_{w c}{ }^{0,0}\left(\mathbf{k}_{\|}\right) R_{w S}{ }^{0,0}\left(\mathbf{k}_{\|}\right) \mathrm{e}^{2 j k_{z L} a}},
$$


(a)

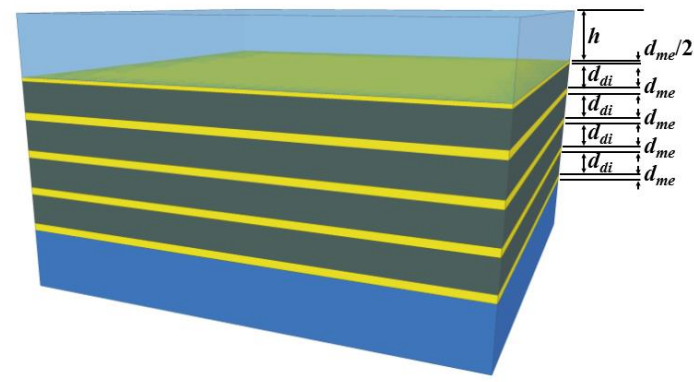

Silver $(\mathrm{Ag})$

Silicon Dioxide $\left(\mathrm{SiO}_{2}\right)$

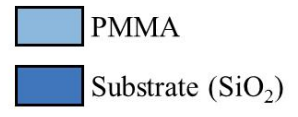

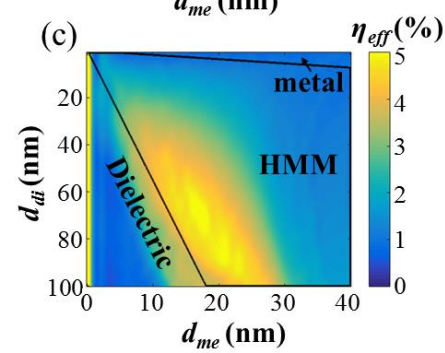

Figure 5.4. (a) $\mathrm{HMM}$ consisting of 9 alternating $\mathrm{Ag} / \mathrm{SiO}_{2}$ layers on top of $\mathrm{SiO}_{2}$ substrate. The PMMA cover layer with thickness $h$ is positioned on top of the HMM. The (b) and (c) maps show the values of $h$ and $\eta_{\text {eff, }}$ respectively, as function of the metal $\left(d_{m e}\right)$ and dielectric $\left(d_{d i}\right)$ thicknesses required to achieve $P=10$. The black lines in (b) and (c) separate the regions where the stack behaves as a metal, a HMM and a dielectric, according to [101].

$$
B^{\chi}\left(\mathbf{k}_{\|}\right)=\frac{E_{L}^{\chi,-}\left(\mathbf{k}_{\|}\right)+E_{L}^{\chi,+}\left(\mathbf{k}_{\|}\right) R_{w C \chi, \chi}{ }^{0,0}\left(\mathbf{k}_{\|}\right) \mathrm{e}^{2 j k_{z L} b}}{1-R_{w C \chi, \chi}{ }^{0,0}\left(\mathbf{k}_{\|}\right) R_{w S, \chi, \chi}{ }^{0,0}\left(\mathbf{k}_{\|}\right) \mathrm{e}^{2 j k_{2 L} a}},
$$

Moreover, since neither $\eta_{\alpha}$ nor $\Gamma_{\alpha}$ depends on the QE's position along the $x$ and $y$ axes, the triple integral in (5.35) is reduced to a single integral along the $z$-axis only. The thickness $h$ required to achieve $P=10$ is obtained as function of $d_{d i}$ and $d_{m e}$ by solving (5.40) and (5.41) and then substituting the results into (5.35). Figure 5.4 (b) shows the resulting map for $0 \mathrm{~nm}<d_{d i} \leq 100 \mathrm{~nm}$ and $0 \mathrm{~nm} \leq d_{m e} \leq 40 \mathrm{~nm}$. Note that the total radiated power increases with $h$ because more QEs can be embedded into the PMMA layer in this case. The map corresponding to $\eta_{\text {eff }}$ (shown in Figure 5.4 (c)) is calculated using the value of $h$ obtained from Figure $5.4(\mathrm{~b})$. The black lines in Figure $5.4(\mathrm{~b}, \mathrm{c})$ separate the maps into dispersion regions where the stack behaves as a dielectric, an HMM, and a metal according to [101]. The maximum values of $\eta_{\text {eff }}$ and $h$ in both figures occur close to the transition region where the stack changes its behavior from HMM to dielectric. In this region, the permittivity tensor elements $\varepsilon_{x}$ and $\varepsilon_{y}$ (obtained with [101]) not only change their signs from negative to positive, but they are also close to zero. According to [40], $P$ is higher in regions where both $\varepsilon_{x}$ and $\varepsilon_{y}$ are simultaneously close to zero and negative, which is consistent with the results shown in Figure $5.4(\mathrm{~b}, \mathrm{c})$. Note also that, despite the claim that metals outperform HMMs [158]-[160], in the present configuration the best performance occurs when the stack behaves as an HMM, as indicated by the high $\eta_{\text {eff }}$ in this region (see Figure 5.4 (c)). This optimized performance gives $\eta_{\text {eff }}=4.7 \%$ for $d_{d i}=60 \mathrm{~nm}, d_{m e}=15 \mathrm{~nm}$ and $h=40 \mathrm{~nm}$. 


\subsubsection{Step 2: NPHM period and fill-factor optimization with the 2D- SAM}

After calculating both the host and HMM layer thicknesses, the next step is to calculate the NPHM optimum period $\left(\Lambda_{x}\right)$ and fill factor $f_{2 D}$ for the system shown in Figure 5.5 (a). To do so, we treat the system as $2 \mathrm{D}$ so that $\mathrm{d} / \mathrm{d} y=k_{y}=K_{y}=0$ [124]. This guarantees that only TM modes are excited, as obtained after substituting $k_{y}=0$ into (5.20) and (5.21). It also guarantees that only in-plane solutions of $R_{w \Delta}^{i, m}$ are analyzed since this coefficient now depends only on $k_{x}$ (rather than on both $k_{x}$ and $k_{y}$ ), $N_{y}=1$ and there is no crosspolarization [124]. Consequently, the time required to calculate $R_{w \Delta_{\chi}, \sigma}^{i, m}$ is markedly reduced compared to that of a 3D system when using RCWA. This feature makes the 2D method a good first approximation to hasten the optimization of more realistic 3D systems. Note that a $2 \mathrm{D}$ dipole source behaves equivalently to a dipole line in $3 \mathrm{D}$, so it is denoted here as 2D-dipole. After these assumptions, (5.11) is rewritten as follows,

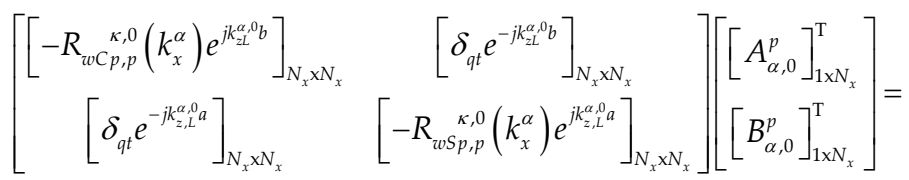

$$
\begin{aligned}
& {\left[\begin{array}{l}
{\left[R_{w w C p, p}^{\kappa, 0}\left(k_{x}^{\alpha}\right)\right]_{N_{x} \times N_{x}}\left[E_{L, \alpha, 0}^{p,+} \mathrm{e}^{j k_{z, L}^{\alpha, 0} b}+E_{S w, \alpha, 0}^{p} \mathrm{e}^{j k_{z L}^{\alpha, 0} h}\right]_{1 \times N_{x}}-\left[E_{C w, \alpha, 0}^{p}\right]_{1 \times N_{x}}^{\mathrm{T}}} \\
{\left[R_{w w p, p}{ }^{\kappa, 0}\left(k_{x}^{\alpha}\right)\right]_{N_{x} \times N_{x}}\left[E_{L, \alpha, 0}^{p,-} \mathrm{e}^{j k_{z, L}^{\alpha, 0} a}+E_{C w, \alpha, 0}^{p} \mathrm{e}^{j k_{z L}^{\alpha, 0} h}\right]_{1 \times N_{x}}-\left[E_{S w, \alpha, 0}^{p}\right]_{1 \times N_{x}}^{\mathrm{T}}}
\end{array}\right] .}
\end{aligned}
$$

In this procedure, the 2D-dipole embedded in the $40 \mathrm{~nm}$ thick host PMMA layer is oriented parallel to the HMM surface, i.e., along the $x$-axis $(\mathbf{p}=\mathbf{p} \mathbf{x})$. This occurs because $2 / 3$ of the QEs are oriented parallel to the HMM surface ( $x$ and y axes) in the 3D case [37]. Although arbitrarily distributed in the host layer, the QEs' position affects the amplitude and phase of the evanescent fields that interact with the NPHM surface, but not with the excited modes. Note that optimizing $\Gamma_{x} / \Gamma_{0}$ and $\eta_{x}$ at one position is enough to guarantee that both parameters are optimized for any position inside the host layer because the outcoupling mechanism depends mostly on the NPHM modes. In this sense, we assume the 2D-dipole centered at the NPHM ridge at $a=10 \mathrm{~nm}$ from the NPHM surface because $\Gamma_{x} / \Gamma_{0}$ is higher in this case [55]. Assuming $0.5 \leq f_{2 D} \leq 0.99$ and $50 \mathrm{~nm} \leq \Lambda_{x} \leq 500 \mathrm{~nm}$, we obtain the $\Gamma_{x} / \Gamma_{0}$ and $\eta_{x}$ maps shown in Figure $5.5(\mathrm{~b}, \mathrm{c})$, respectively. Figure $5.5(\mathrm{~b}, \mathrm{c})$ also present $f_{3 D}$ for a groove width $\left(\rho_{g}\right)$ of $50 \mathrm{~nm}$ (white lines) to facilitate an eventual fabrication process. Observe in these figures that the $\Gamma_{x} / \Gamma_{0}$ peak values correspond to four resonant modes indicated by the dashed lines for ridge widths $\rho_{r}=42 \mathrm{~nm}(\mathrm{~A}), 156 \mathrm{~nm}$ (B), $278 \mathrm{~nm}$ (C) and $400 \mathrm{~nm}$ (D). These resonances are not function of $\rho_{g}$, but of $\rho_{r}$. This behavior suggests that the modes are confined in the NPHM ridge, which is a signature of localized surface plasmon polaritons (LSPP). At the resonance condition, $\Gamma_{x} / \Gamma_{0}$ is highly enhanced, reaching values as high as 80 according to Figure 5.5 (c). However, the high ohmic losses associated with LSPPs attenuates most of the coupled energy and reduces the amount of NPHM power radiated into free-space. Consequently, $\eta_{x}$ is minimum at the LSPP 
(a)

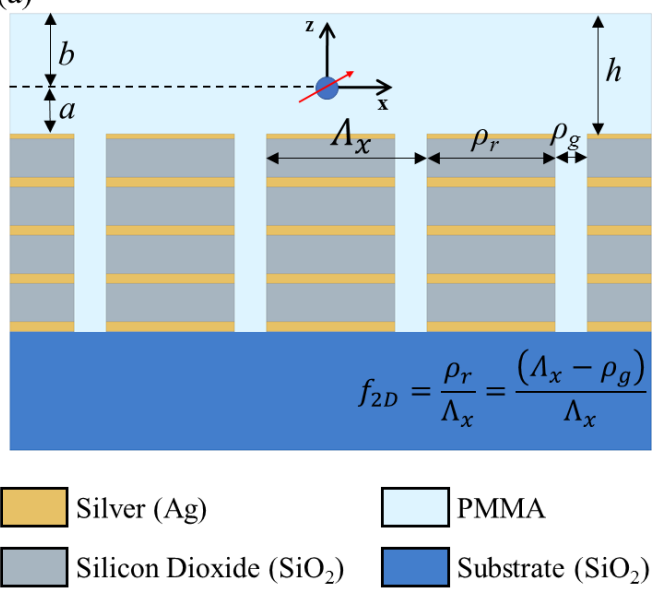

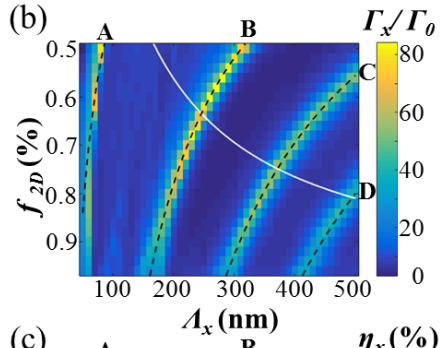

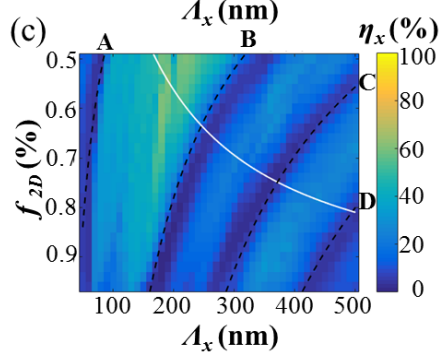

Figure 5.5. (a) $2 \mathrm{D}-\mathrm{NHMM}$ consisting of 9 alternating $\mathrm{Ag} / \mathrm{SiO}_{2}$ layers with period $\Lambda_{x}$ and fill factor $f_{2 D}$ on top of a $\mathrm{SiO}_{2}$ substrate. The PMMA host layer with thickness $h$ is on top of the NHMM. (b) and (c) are the $\Gamma_{x} / \Gamma_{0}$ and $\eta_{x}$ maps, respectively, as function of $\Lambda_{x}$ and $f_{2 D}$ of a 2D-dipole placed at the center of the ridge at a distance $a=10 \mathrm{~nm}$ from the NPHM surface. The dashed black lines in (b) and (c) represent the LSPP, while the white lines represent the $f_{3 D}$ of a 3D structure with $\rho_{8}=50 \mathrm{~nm}$.

resonance, as also indicated by equation (5.35) and Figure 5.5 (c). This suggests a compromise between $\Gamma_{x} / \Gamma_{0}$ and $\eta_{x}$ which is achieved with $\rho_{g}=50 \mathrm{~nm}, \Lambda_{x}=230 \mathrm{~nm}$ and $f_{2 d}$ $=0.6$, resulting in $\Gamma_{x} / \Gamma_{0}=28$ and $\eta_{x}=27 \%$.

\subsubsection{Step 3: NPHM fine tuning with the 3D-SAM}

We now use the optimized 2D parameters estimated in the previous section as a first approximation for the 3D model. First, we calculate $\eta_{\alpha}$ and $\Gamma_{\alpha} / \Gamma_{0}$ at each possible position inside the host layer (to be used in the calculation of the system's $\eta_{\text {eff }}$ and $P$ ). We investigate three different structures to demonstrate the potential of the proposed $3 \mathrm{D}$ model for optimization purposes, namely: structure 1 (S1), consisting of a non-patterned HMM with thicknesses $d_{d i}=60 \mathrm{~nm}, d_{m e}=15 \mathrm{~nm}$, see Figure 5.6 (a); structure 2 (S2), which is structure S1 patterned along the $x$-axis (1D-NPHM) with period $\Lambda_{x}=230 \mathrm{~nm}$ and $\rho_{g}=50$ $\mathrm{nm}$, see Figure 5.6 (e); and structure 3 (S3), which is structure S1 patterned along the $x$ and $y$ axes (2D-NPHM) with period $\Lambda_{x}=\Lambda_{y}=230 \mathrm{~nm}$ and $\rho_{g}=50 \mathrm{~nm}$, as seen in Figure 5.6 (i). For all three cases the host thickness is set to $h=40 \mathrm{~nm}$. The reflection and transmission coefficients $\left(v_{\chi, \sigma}^{i, m}\left(k_{x}, k_{y}\right), v=R_{w c}, R_{w s}\right.$ or $\left.T_{w c}\right)$ are calculated using the 3D-RCWA for $\left(N_{x}, N_{y}\right)$ $=(1 \times 1),(15 \times 1)$ and $(15 \times 15)$ relative to S1, S2 and S3, respectively. The dipole is simulated assuming the parallel wavevector components of the incident plane wave $\left(k_{x}\right.$ and $\left.k_{y}\right)$ are swept by 1801 points each in the interval $0 \leq\left|k_{x} / k_{0}\right|,\left|k_{y} / k_{0}\right| \leq 20$. After calculating $v_{\chi, \sigma}^{i, m}\left(k_{x}^{0}, k_{y}^{0}\right), \Psi_{c o m b}^{\chi}$ is computed by solving the linear system (11) in the intervals $0 \leq k_{x}^{0} \leq K_{x}$ 

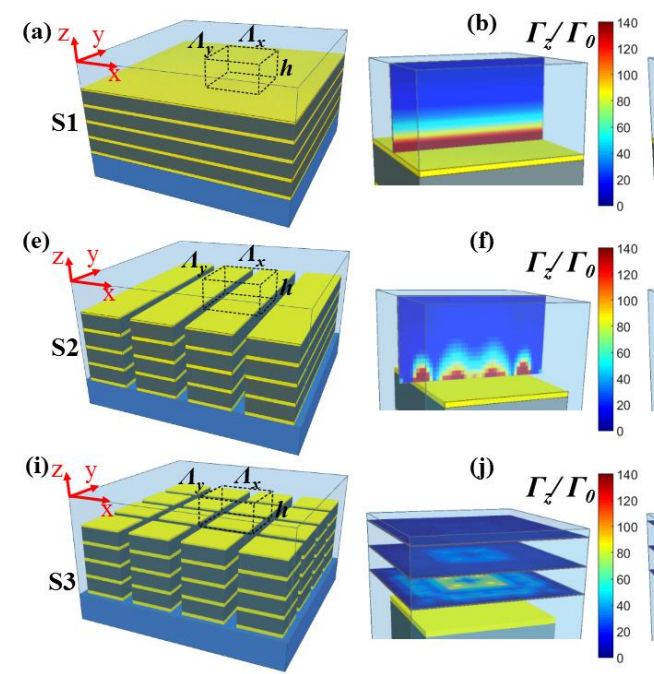

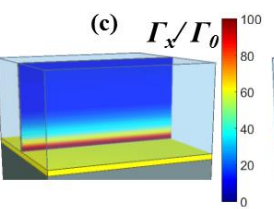

(g)

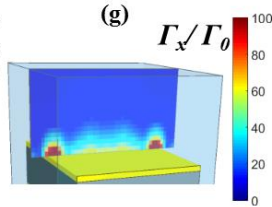

(k)

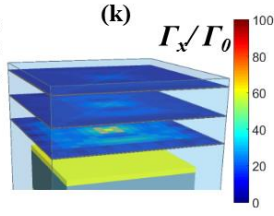

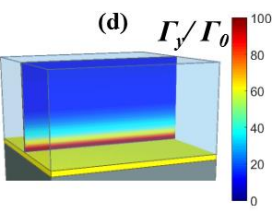

(h)

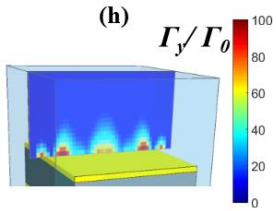

(l)

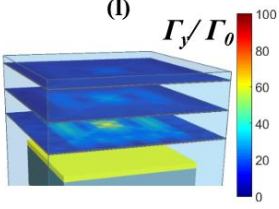

Figure 5.6. Schematics of the three NPHM analyzed with the 3D-SAM: (a) S1 - non-patterned, (e) S2 - patterned along the $x$-axis and (i) S3 - patterned along the $x$ - and $y$-axes. $\Gamma_{\alpha} / \Gamma_{0}$ as function of position inside the PMMA layer for structures $\mathrm{S} 1(\mathrm{~b}, \mathrm{c}, \mathrm{d}), \mathrm{S} 2(\mathrm{f}, \mathrm{g}, \mathrm{h})$ and S3 $(\mathrm{j}, \mathrm{k}, \mathrm{l})$ assuming $\alpha=z(\mathrm{~b}, \mathrm{f}, \mathrm{j}), x(\mathrm{c}, \mathrm{g}, \mathrm{k})$ and $y(\mathrm{~d}, \mathrm{~h}, \mathrm{l})$.

and $0 \leq k_{x}^{0} \leq K_{y}$. Finally, $\Psi_{\text {comb }}^{\chi}$ is used into (5.5) to obtain $\Psi x$, which is then used in (5.22) to obtain $\Gamma_{\alpha} / \Gamma_{0}$.

Figure 5.6 shows $\Gamma_{\alpha} / \Gamma_{0}$ as function of position for structures S1 (a-d), S2 (e-h) and S3 (i-l). The dipole is assumed oriented along $\alpha=z(\mathrm{~b}, \mathrm{f}, \mathrm{j}), x(\mathrm{c}, \mathrm{g}, \mathrm{k})$ and $y(\mathrm{~d}, \mathrm{~h}, \mathrm{l})$. Note that $\Gamma_{\alpha} / \Gamma_{0}$ for S1 and S2 is plotted in the $x z$ plane because there are no variations along the $y$-axis. In contrast, $\Gamma_{\alpha} / \Gamma_{0}$ for S3 is plotted in three different planes, namely $z=-5,-20$ and $-35 \mathrm{~nm}$ because the patterns are along the $x$ and $y$ axes. In all cases, $\Gamma_{\alpha} / \Gamma_{0}$ increases exponentially as the QE approaches the NPHM/HMM interface due to strong evanescent field coupling with the structure. Moreover, when $\mathbf{p}=p \mathbf{z}$ the QE only radiates TM polarized waves (see (5.20) and (5.21)). In contrast, when $\mathbf{p}=p \mathbf{x}$ or $p \mathbf{y}$ the QE radiates both TE and TM polarized waves. Note that $\Gamma_{z} / \Gamma_{0}$ is higher than $\Gamma_{x} / \Gamma_{0}$ and $\Gamma_{y} / \Gamma_{0}$, as shown in Figure 5.6, because the hyperbolic dispersion profile occurs only for TM polarization [101]. Note also that $\Gamma_{\alpha} / \Gamma_{0}$ of $\mathrm{S} 1$ is constant (and identical for $\alpha=x$ or $y$ due to symmetry) in the $x y$-plane regardless of the polarization (see Figure $5.6(\mathrm{~b}-\mathrm{d})$ ) owed to its uniformity along the $x$ and $y$ directions. In contrast, $\Gamma_{\alpha} / \Gamma_{0}$ of S2 varies along $x$ and is maximum close to the ridge's edge due to scattering, as seen in Figure 5.6 (f-h). Moreover, the reduced coupling of evanescent waves due to QEs located far from the groove (for S2 or S3) results in a decrease of $\Gamma_{\alpha} / \Gamma_{0}$. Regarding S3, $\Gamma_{\alpha} / \Gamma_{0}$ is maximum at the center of the NPHM for $\alpha=x$ (Figure $5.6(\mathrm{k})$ ) or $\alpha$ $=y($ Figure $5.6(\mathrm{l}))$, while it is minimum for $\alpha=z$ (Figure $5.6(\mathrm{j})$ ). Note the similarity of $\Gamma_{z} / \Gamma_{0}$ (Figure $5.6(\mathrm{f}, \mathrm{j})$ ) and $\Gamma_{y} / \Gamma_{0}$ (Figure $5.6(\mathrm{~h}, \mathrm{l})$ ) relative to S2 and S3, respectively. The same pattern is observed for $\Gamma_{x} / \Gamma_{0}$ if $\mathrm{S} 2$ is rotated $90^{\circ}$ around the $z$-axis. 
Next, $\eta_{\alpha}$ is calculated as function of position inside the host layer using (5.35) for structures S1 (a- c), S2 ( (d-f)) and S3 (g-i) assuming $\alpha=z(\mathrm{a}, \mathrm{d}, \mathrm{g}), x(\mathrm{~b}, \mathrm{e}, \mathrm{h})$, and $y(\mathrm{c}, \mathrm{f}, \mathrm{i})$, as shown in Figure 5.7. According to (5.35), $\eta_{\alpha}$ is inversely proportional to $\Gamma_{\alpha} / \Gamma_{0}$ and thus $\eta_{\alpha}$ increases when the QE is located further away from the NPHM surface, as shown in Figure 5.7 for S1, S2, and S3. Furthermore, when $h<<\lambda$, the interference at the QE position between the wave radiated by the QE $\left(\left|\mathbf{k}_{\|}\right| \leq k_{0}\right)$ and the wave reflected from the NPHM surface is destructive for $\mathbf{p}=p \mathbf{z}$ and constructive for $\mathbf{p}=p \mathbf{x}$ or $p \mathbf{y}$. The combination of high $\Gamma_{\alpha} / \Gamma_{0}$ with destructive interference makes $\eta_{z}$ smaller than $\eta_{x}$ and $\eta_{y}$, as observed in Figure 5.7. In fact, $\eta_{z}$ is close to $0 \%$ (Figure 5.7 (a)) for any QE position for S1. In contrast, $\eta_{x}$ and $\eta_{y}$ are as high as $40 \%$ (Figure $5.7(\mathrm{~b}, \mathrm{c})$ ) for QEs far from the NPHM interface. The nanopatterning present in S2 and S3 convert the evanescent waves $\left(\left|\mathbf{k}_{\|}\right| \geq k_{0}\right)$ inside the NPHM into propagating waves $\left(\left|\mathbf{k}_{\|}\right| \leq k_{0}\right)$, therefore increasing $\eta_{\alpha}$ for QEs located at any position inside the host layer, especially for those close to the NPHM surface. As expected, $\eta_{z}$ and $\eta_{y}$ of S2 increase when the QE is located above the NPHM groove but distant from the NPHM surface (see Figure $5.7(\mathrm{~d}, \mathrm{f})$ ), since these are the positions where $\Gamma_{\alpha} / \Gamma_{0}$ is minimum. Similarly for S3, the position where $\eta_{\alpha}$ is maximum coincides with the positions where $\Gamma_{\alpha} / \Gamma_{0}$ is minimum, as seen in Figure 5.7 (g,i).

Once $\Gamma_{\alpha} / \Gamma_{0}$ and $\eta_{\alpha}$ have been obtained, the next step is to calculate the $\eta_{\text {eff }}$ and $P$ using (5.35) and (5.36). However, increasing $P$ is not a trivial task because $\Gamma_{\alpha} / \Gamma_{0}$ is weighed by $\eta_{\alpha}$. Thus, mapping $\eta_{\alpha}$ and $\Gamma_{\alpha} / \Gamma_{0}$ at each position inside the host layer is essential to simplify this process and may also prove to be a useful tool for understanding the system's overall behavior. Usually, this mapping is carried out numerically with computationally costly methods such as FDTD [55], [114]. For example, in a regular desktop computer (Intel Core

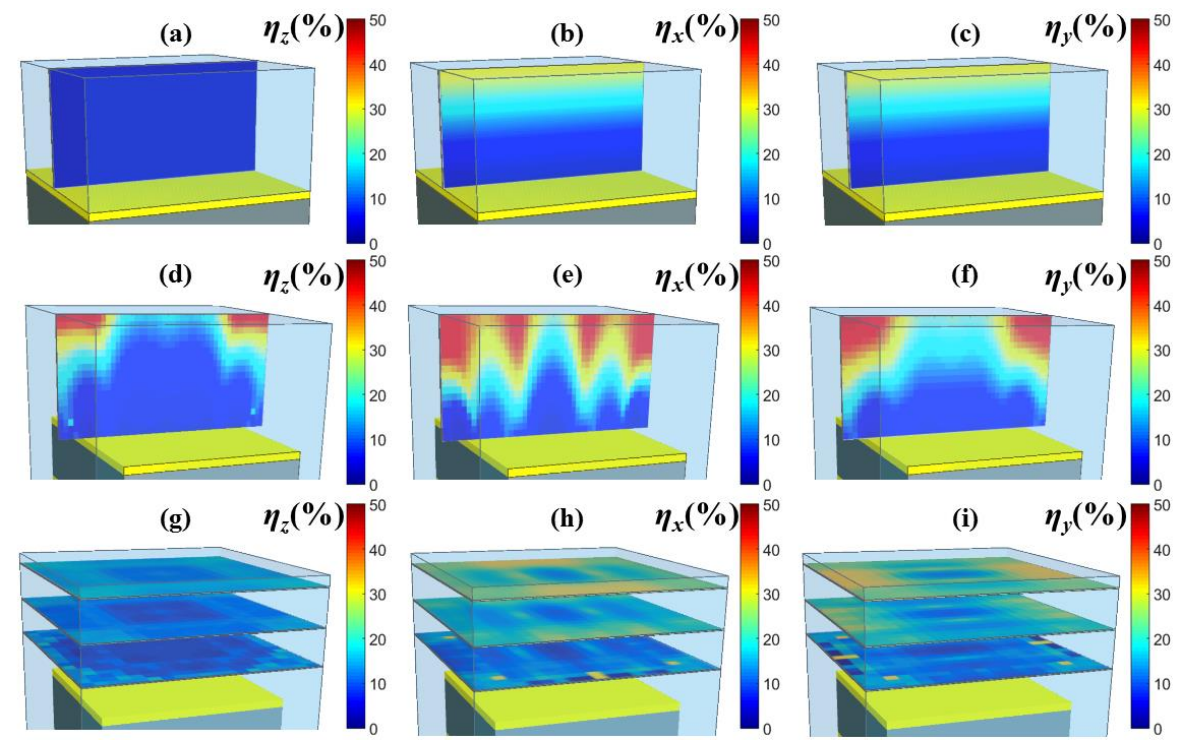

Figure 5.7. $\eta_{\alpha}$ as function of position inside the host layer on top of structures S1 (a,b,c), S2 (d,e,f) and S3 (g,h,i) assuming $\alpha=z(\mathrm{a}, \mathrm{d}, \mathrm{g}), x(\mathrm{~b}, \mathrm{e}, \mathrm{h})$ and $y(\mathrm{c}, \mathrm{f}, \mathrm{i})$. 
i7-3960X processor with 64GB of RAM), each 3D-FDTD simulation of structure S2 takes approximately 20 hours. Therefore, approximately 180 days would be required to build the maps for each polarization (each map size is $24 \times 18$ pixels, but only $12 \times 18$ pixels are solved due to symmetry). This is reduced to approximately seven days for all three polarizations with the proposed model (4 days to calculate all $v_{\chi, \sigma}^{i, m}$ terms and 20 minutes for each pixel), which corresponds to about a $96 \%$ reduction in the processing time.

The map building for structure S3, in contrast, is computationally more intensive since it is nano-patterned along both $x$ and $y$ axes. With the 3D-FDTD method, approximately 906 days would be necessary to obtain the maps of all three polarizations of Figure 5.6 and Figure 5.7 (each map cut is $21 \times 21$ pixels, but only $11 \times 11$ pixels are solved due to symmetry). However, with our approach $v_{\chi, \sigma}^{i, m}$ is calculated in 4 days and each pixel is calculated in approximately 3 hours, resulting in a total simulation time of 19 days for all three polarizations. In summary, with the proposed method the computational time for simulating 1D and 2D NPHMs is reduced by $96 \%$ and $98 \%$, respectively, when compared to FDTD. This allows $P$ and $\eta_{\text {eff }}$ to be calculated more efficiently as function of $h$.

The last parameter to be optimized is the host layer thickness $h$ since it is directly related to $P$ and $\eta_{\text {eff. }}$. The plots of $P$ and $\eta_{\text {eff }}$ for $10 \mathrm{~nm} \leq h \leq 50 \mathrm{~nm}$ are shown, respectively, in Figure $5.8(\mathrm{a}, \mathrm{b})$ for structures S1 (blue line), S2 (squares) and S3 (circles). The number of simulated $h$ points differs for each structure because of the time required to map $\eta_{\alpha}$ and $\Gamma_{\alpha} / \Gamma_{0}$. According to Figure 5.8 (a), a high $P$ value is achieved for small $h$ due to the QEs strong evanescent wave coupling to the HMM surface in this situation. This figure also shows that S1's $P$ is higher than those from S2 and S3, particularly for small $h$. The relatively small $P$ of S2 and S3 is due to the low $\Gamma_{\alpha} / \Gamma_{0}$ and high $\eta_{\alpha}$ regions (see (5.35) and (5.36)) for QEs close to the HMM surface but above the grooves of these structures, as shown in Figs. 6 and 7. Note that part of the QEs is located far from the NPHM surface when $h$ is increased, causing the NPHM influence on the QE decay to reduce and, consequently, the $P$ of all structures to converge to the same value, as observed in Figure 5.8 (a). In contrast, S1's $\eta_{\text {eff }}$ diverges from those of S2 and S3 as $h$ increases, as shown in Figure 5.8 (b). Moreover, the lack of nano-patterning in $S 1$ not only increases $P$, but also reduces $\eta_{\text {eff, }}$ as evidenced inFigure $5.8(\mathrm{~b})$, since no high- $k$ modes decoupling mechanism
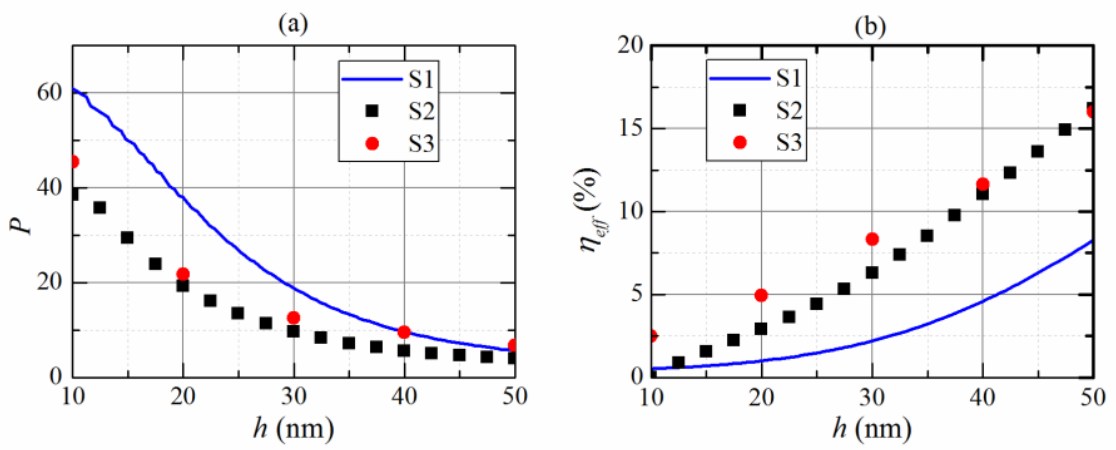

Figure 5.8. Calculated $P(\mathrm{a})$ and $\eta_{\text {effl }}(\mathrm{b})$ for structures S1 (blue line), S2 (squares) and S3 (circles) for $10 \mathrm{~nm} \leq h \leq 50 \mathrm{~nm}$. 
exists inside the HMM in this case. However, S3's nano-patterning decouples the high- $k$ modes that propagate along the $x$ and $y$ axes, whereas S2's decouples only those propagating along the $x$-axis resulting in $\eta_{\text {eff }}$ higher for S3 than for S2. This effect is more evident for $h<40 \mathrm{~nm}$ due to the more efficient coupling of evanescent waves with the high$k$ modes. According to Figure 5.8 (a) and $8(\mathrm{~b})$, it is possible to achieve a good compromise between $P$ and $\eta_{\text {eff }}$ with an appropriate choice of $h$, which is computationally viable only with the proposed method.

Finally, the NPHM designs relative to structures S1, S2, and S3 that produce $P=10$ with high $\eta_{\text {eff }}$ require $h=40 \mathrm{~nm}\left(\eta_{\text {eff }}=4.3 \%\right), 30 \mathrm{~nm}\left(\eta_{\text {eff }}=7.3 \%\right)$, and $40 \mathrm{~nm}\left(\eta_{\text {eff }}=11.6 \%\right)$, respectively. Therefore, in addition to reducing the computational time, the proposed optimization procedure helps to increase the QEs' quantum efficiency by about $69 \%$ and $170 \%$ using 1D and 2D NPHMs, respectively.

\subsection{Chapter conclusion}

In this chapter, we have proposed a semi-analytical method capable of calculating, both in $2 \mathrm{D}$ and $3 \mathrm{D}$, all radiation parameters of any electromagnetic source type (including QEs modeled as a dipole) embedded in any stratified media (nano-patterned or not). This method also allows for multiple electromagnetic sources to be arbitrarily distributed in the cover, substrate, and any inner $\left(\mathrm{w}^{\text {th }}\right)$-layer and used simultaneously. The validation procedure consisted in calculating $\Gamma_{\Delta}$ and $Q_{c}$ for a two-source system consisting of QEs (inside a host bounded by nano-patterned layers, $\Delta=\mathrm{L}$ ) and a dipole source (in the cover layer, $\Delta=\mathrm{C}$ ). The influence of the former on the latter was also investigated and compared with FDTD simulations. We were also able to map $\Gamma_{\alpha}$ and $\eta_{\alpha}$ as function of the QE and NPHM relative position, which is only feasible because of the method's low computational cost. This mapping is an important tool for understanding the decay behavior of the whole system due to the random distribution/orientation nature of QEs in NPHMs. We were also able to calculate analytically the decay curve $g(t)$ of a multisource system, which is fundamentally different from the exponential decay fitting and exponential summation techniques normally adopted in the literature. Besides allowing us to calculate $\eta_{\text {eff }}$ and $\Gamma_{\text {eff }}$ more efficiently, this feature also allowed us to propose a new optimization procedure to maximize $\eta_{\text {eff }}$ for the desired $\Gamma_{\text {eff. }}$. The optimization procedure was divided into three steps: 1) a non-patterned HMM was used to optimize the metallic and dielectric layer thicknesses with the proposed 3D-SAM. Here, we took advantage of the structure's axial symmetry and lack of nano-patterns to reduce the 3D-SAM computational cost even further and thus calculate $\Gamma^{f p}$ and $\eta^{f p}$ maps required to calculate $\eta_{\text {eff }}$ and $\left.\Gamma_{\text {eff }}, 2\right)$ the computationally efficient 2D-SAM model was then employed to optimize the nanopattern's period and fill-factor; 3 ) finally, the 2D optimized parameters were fed into the 3D-SAM model for final tuning. This procedure was applied to maximize the $\eta_{\text {eff }}$ of Rhodamine 6G (R6G) with a tenfold increase in $\Gamma_{\text {eff. }}$. We showed that an NPHM consisting of 9 alternating layers of $\mathrm{Ag}$ and $\mathrm{SiO}_{2}$ could enhance $\eta_{\text {eff }}$ from $4.3 \%$ (non-patterned) up to $7.3 \%$ and $11.6 \%$ if patterned along one (1D-NPHM) and two axes 
(2D-NPHM), respectively. We also showed that the computational time required to build the $\Gamma_{\alpha}$ and $\eta_{\alpha}$ maps could be reduced by $96 \%$ (1D NPHMs) and 98\% (2D NPHMs) when compared to FDTD simulations. 



\section{Chapter 6 \\ Conclusion and \\ Perspectives}




\section{Chapter 6 - Summary}

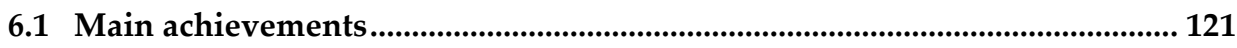

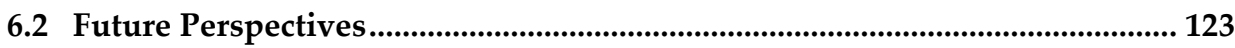

6.2.1 Nano-patterned hyperbolic metamaterials for telecommunication applications 124

6.2.1 Nano-patterned hyperbolic metamaterials for sensing applications ... 125 


\subsection{Main achievements}

In this thesis, we have advanced the current state-of-the-art of two key subjects, namely, homogenization of HMM with spatial dispersion analysis and characterization of the radiation parameters of QEs inside nano-patterned HMM by means of the development of novel and efficient semi-analytical modeling methods. These two key proposed entailed additional contributions and achievements that can be further subdivided into four major parts: new parameters retrieval in which the spatial dispersion is taken into consideration (chapter 2); semi-analytical model to calculate in both 2D and 3D the emission parameters of QEs embedded on top or under any nano-patterned structure (chapter 4); generalization of the previously model so that the QEs can be embedded inside the NPHM (chapter 5); finally, a semi-analytical method to calculate the decay curve. These contributions are detailed bellow:

\section{1) New homogenization of uniaxial metamaterials with spatial dispersion (Chapter}

\section{2)}

We have proposed a new parameter retrieval approach in which all electromagnetic parameters of the medium are obtained and where spatial dispersion is properly taken into account. We have also relaxed the constraint on nonmagnetic metal/dielectric metamaterials. This approach was successfully applied to different metal/dielectric stacks in order to address the effects of the layer thickness, number of layers, and metal/dielectric choice on the spatial dispersion. The results have demonstrated that all investigated metal/dielectric stacks have a clear magnetic response, particularly for thicker layers. Moreover, for large $\left|k_{x} / k_{0}\right|$ the dispersion curve shows a flat region with a large imaginary part that arises due to the absence of propagating modes inside the metamaterial. This flat region is also strongly dependent on the thickness of the layers. The thicker the metal/dielectric layer is, the sooner (for lower $\left|k_{x} / k_{0}\right|$ values) the flat and lossy region appears. This behavior is a direct manifestation of the spatial dispersion and strongly affects the accuracy with which the Purcell factor is calculated. In order to illustrate this issue, we have calculated the Purcell factor for $\mathrm{Rb}$ atoms $10 \mathrm{~nm}$ above the surface of a $\mathrm{Ag} / \mathrm{TiO}_{2}$ stack with two distinct filling factors, i.e., $f f=0.3$ and $f f=0.5$, and $N=13$ layers, for two emission wavelengths, i.e., $422 \mathrm{~nm}$ and $780 \mathrm{~nm}$. The results were compared with three different approaches, namely, the benchmark result, the approach in [81], and the EMT approach. We have shown that if spatial dispersion is not properly taken into account, the Purcell factor is overestimated, as observed with both [81] and EMT approaches. However, the CPR approach has shown excellent agreement with the benchmark results.

\section{2) Proposing a HMM capable of enhancing the Purcell factor of Cy7 (chapter 3)}

In chapter 3, we have proposed an HMM capable of enhancing the Purcell factor of the fluorescent molecules (Cy7). These molecules are embedded in a thin PMMA host film deposited on the top of the HMM. This structure was modeled with a new 
mathematical formalism to calculate $P$ and $\eta$ taking into account the reflections at the upper and bottom host interfaces. Moreover, we have also proposed an analytical method to calculate $g(t)$ as function of the QE's position inside the host layer. We demonstrated that QEs placed far from the HMM surface have lower $P$ but higher $\eta$. Thus, they contribute more to $g(t)$ than those closer to the HMM surface. Finally, we have shown that the host thickness has a direct influence on the system's $g(t)$, and that with an adequate choice of $h$ it is possible to control the systems decay behavior.

\section{3) Design and analysis of grating-assisted radiation emission of QEs in HMM} (chapter 4)

In this thesis, we have proposed a novel semi-analytical model to investigate the radiation emission profile of QEs under a nano-patterned metamaterial. In addition, the reduced computational burden of this method (compared to numerical approaches) allows the Purcell factor and the radiated power to be calculated in a more straightforward manner. The model was applied to a HMM composed of seven $\mathrm{Ag} / \mathrm{TiO}_{2}$ layers with a silver grating on its top. Using a $2 \mathrm{D}$ configuration first, we optimized the grating geometrical parameters and showed that the radiation pattern is highly directive for $\Lambda_{x}=420 \mathrm{~nm}$. Furthermore, the normalized power coupled from high- $k$ HMM modes into air was increased to 1.67 for perpendicular polarization for a fill factor $f=70 \%$ and grating height $a=80 \mathrm{~nm}$. With such optimum parameters, we calculated $\Psi_{\alpha}^{2 D}$ and $P_{\alpha}^{2 D}$ as function of the $\mathrm{QE}$ relative position with respect to the grating groove and ridge. The maximum $\Psi_{\alpha}^{2 D}$ and $P_{\alpha}^{2 D}$ values occurred at the same QE relative position, which helped increase the system's overall Purcell factor, according to (28). After the 2D optimization procedure, we applied the optimized parameters to a 3D periodic array of nano-cylinders. We observed that the radiation pattern lost some of its directivity because of cross-polarized transmission; however, the QE showed the highest Purcell factor $\left(P_{z}=145\right.$ and $P_{x}=$ $67.5)$ and the highest radiated power $\left(\Psi_{z}^{3 D}=2\right.$ and $\left.\Psi_{x}^{3 D}=0.75\right)$ for $q=10 \mathrm{~nm}$. Moreover, in comparison with the HMM without the gratings, we have demonstrated that our approach is capable of enhancing the system's quantum efficiency by $240 \%$ if a Purcell factor of 10 is of interest. To the best of our knowledge, the model described in this chapter is the first in the literature capable of mapping, in a detailed and accurate fashion, both the QE s' Purcell factor and total radiated power under or above any nano-patterned structure as function of position.

\section{4) Generalization of the semi-analytical model to include QEs placed inside the nano-patterned Hyperbolic Metamaterials (chapter 5)}

In chapter 5, we have proposed a semi-analytical method capable of calculating, both in $2 \mathrm{D}$ and $3 \mathrm{D}$, all radiation parameters of any electromagnetic source type (including QEs modeled as a dipole) embedded in any stratified media (nano-patterned or not). This method also allows multiple electromagnetic sources arbitrarily distributed in the cover, substrate, and any inner (wth-)layer to be used simultaneously. The validation procedure consisted in calculating $\Gamma_{\Delta}$ and $Q_{c}$ for a two-source system consisting of QEs (inside a host bounded by nano-patterned layers, $\Delta=\mathrm{L}$ ) and a 
dipole source (in the cover layer, $\Delta=\mathrm{C}$ ). The influence of the former on the latter was also investigated and compared with FDTD simulations. We were also able to map $\Gamma_{\alpha}$ and $\eta_{\alpha}$ as function of the QE and NPHM relative position, which was only possible because of the method's low computational cost. This mapping was an important tool for understanding the decay behavior of the whole system due to the random distribution/orientation nature of QEs in NPHMs.

5) Semi-analytical method to calculate the decay curve of QEs embedded in thin polymers layers (chapter 5);

Finally, in this thesis, we have proposed a semi-analytical method capable of calculating, both in $2 \mathrm{D}$ and $3 \mathrm{D}$, all radiation parameters of any electromagnetic source type (including QEs modeled as a dipole) embedded in any stratified media (nano-patterned or not). This method also allows for multiple electromagnetic sources to be arbitrarily distributed in the cover, substrate, and any inner ( $\mathrm{w}^{\text {th }}$ )-layer and used simultaneously. We were able to map $\Gamma_{\alpha}$ and $\eta_{\alpha}$ as function of the QE and NPHM relative position, which is only feasible because of the method's low computational cost. This mapping is an important tool for understanding the decay behavior of the whole system due to the random distribution/orientation nature of QEs in NPHMs. We were also able to calculate analytically the decay curve $g(t)$ of a multi-source system, which is fundamentally different from the exponential decay fitting and exponential summation techniques normally adopted in the literature. Besides allowing us to calculate $\eta_{\text {eff }}$ and $\Gamma_{\text {eff }}$ more efficiently, this feature also allowed us to propose a new optimization procedure to maximize $\eta_{\text {eff }}$ for the desired $\Gamma_{\text {eff. }}$. The optimization procedure was divided into three steps: 1) a non-patterned HMM was used to optimize the metallic and dielectric layer thicknesses with the proposed 3DSAM. Here, we took advantage of the structure's axial symmetry and lack of nanopatterns to reduce the 3D-SAM computational cost even further and thus calculate $\Gamma_{f p}$ and $\eta^{f p}$ maps required to calculate $\eta_{e f f}$ and $\left.\Gamma_{e f f}, 2\right)$ the computationally efficient 2DSAM model was then employed to optimize the nanopattern's period and fill-factor; 3) finally, the $2 \mathrm{D}$ optimized parameters were fed into the 3D-SAM model for final tuning. This procedure was applied to maximize the $\eta_{\text {eff }}$ of Rhodamine 6G (R6G) with a tenfold increase in $\Gamma_{\text {eff. }}$ We showed that an NPHM consisting of 9 alternating layers of $\mathrm{Ag}$ and $\mathrm{SiO}_{2}$ could enhance $\eta_{\text {eff }}$ from $4.3 \%$ (non-patterned) up to $7.3 \%$ and $11.6 \%$ if patterned along one (1D-NPHM) and two axes (2D-NPHM), respectively. We also showed that the computational time required to build the $\Gamma_{\alpha}$ and $\eta_{\alpha}$ maps could be reduced by $96 \%$ (1D NPHMs) and 98\% (2D NPHMs) when compared to FDTD simulations.

\subsection{Future Perspectives}

In this thesis, we have contributed to pave the way towards new approaches to efficiently extract photons from QEs using HMM. The homogenization and semi-analytical methods proposed here allow the optimization of nano-patterned structures aiming at enhancing 


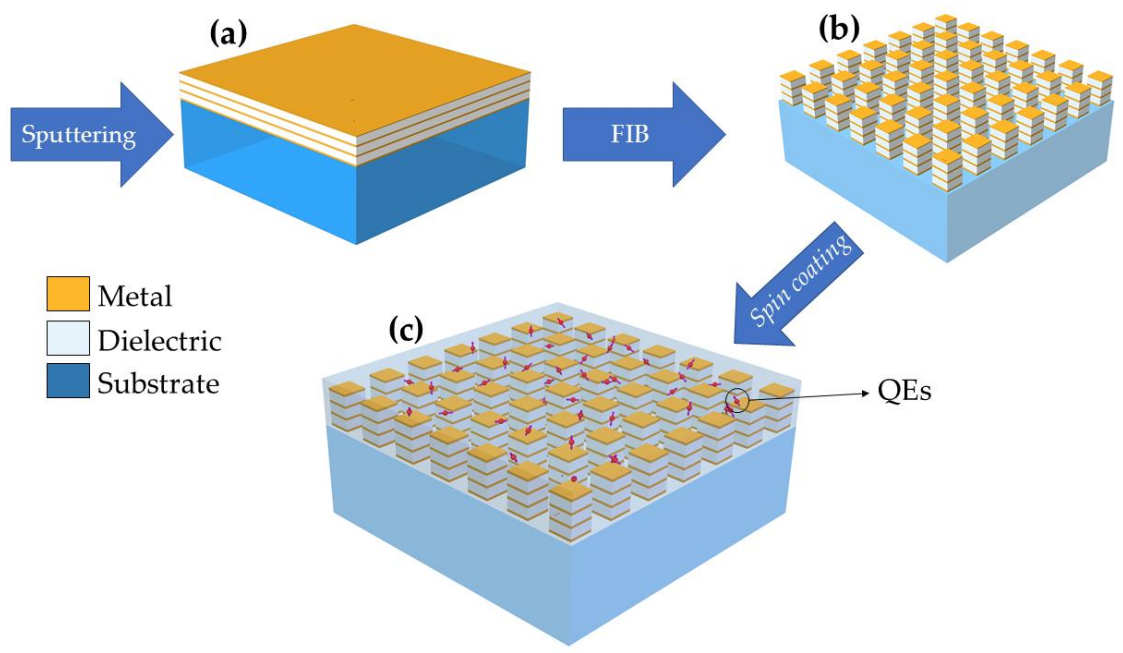

Figure 6.1. NPHM fabrication steps. (a) shows the alternate deposition of the dielectric/metal layers on the top of a substrate. Then, the FIB is used to create the patterned on the deposited layers (b). Finally, the polymer embedded with the QEs are deposited via spin-coat over the NPHM.

the quantum efficiency of QEs. Therefore, this thesis presents the fundamental research towards new light sources. To continuous his research, the $\mathrm{PhD}$ candidate obtained a post-doctoral position (post-doc) at USP with a scholarship from Fundação de Amparo a Pesquisa do Estado de São Paulo (FAPESP). In his post-doc, the student aim to fabricate and characterize the NPHMs designed in this thesis in order to corroborate the optimization procedures. Moreover, the proposed semi-analytical methods can also be used for design NPHM for sensors application, since the high electromagnetic intensity of the high- $k$ modes near the interfaces of these media makes these structures ideal for this purpose. In this sense, we divide the future perspectives in two major areas: NPHM for telecommunication and for sensors applications.

\subsubsection{Nano-patterned hyperbolic metamaterials for telecommunication applications}

After the optimization procedure proposed on this thesis, the NPHM will be fabricated at Physics Institute of São Carlos by Prof. Euclydes Marega. The first stage of the fabrication process consists in alternately evaporate (or sputter) the dielectric and metal layers, as shown in Figure 6.1 (a). Once deposited, the patterns of the HMM are created with a Focused Ion Beam (FIB), resulting in the structure depicted in Figure 6.1 (b). Later, the polymer embedded with the QEs are deposited over the NPHM using a spin-coater machine, as shown in Figure 6.1 (c). For the optical characterization of the structure, Dr. Marega's laboratory have a confocal microscope (Zeiss LSM 780), capable of measuring the lifetime of dyes with high temporal resolution. Moreover, the microscope allows 
performing Fluorescence-lifetime imaging, which is useful for fully characterize the fabricated devices. In addition to HMMs, we will also explore theoretically and experimentally new photonic devices capable of decreasing the QE's lifetime, such as photonics crystals and cavities.

\subsubsection{Nano-patterned hyperbolic metamaterials for sensing applications}

Besides enhancing the Purcell factor of QEs for telecommunication applications, HMMs has attract a great deal of attention over the past years for sensing applications [31], [32], [161], [162]. SPP and LSP based sensors are well known in the literature because of the high field intensity of these modes close to the interface making them extremely sensitive the refractive index variations [163]-[168]. Similarly, the high- $k$ modes of the HMM also present high intensity fields close to the interface, which make them appropriate for sensing applications. In this sense, we aim to utilize the semi-analytical methods proposed in this thesis to optimize the geometrical parameters of NPHM for enhancing the electromagnetic fields at the interfaces. Fortunately, the NPHM fabrication process for sensing applications is similar to the process described in Figure 6.1. Nevertheless, instead of the polymer embedded with QEs, there is a microfluidic channel on the top of the NPHM, as shown in Figure 6.2. The refractive index of the liquid flowing inside the channels are directly related to the NPHM modes. Consequently, a small change in the liquid's refractive index results in a frequency shift of the modes, which changes the structure's reflection $(R)$ coefficient, as seen in inset of Figure 6.2. Therefore, it is possible

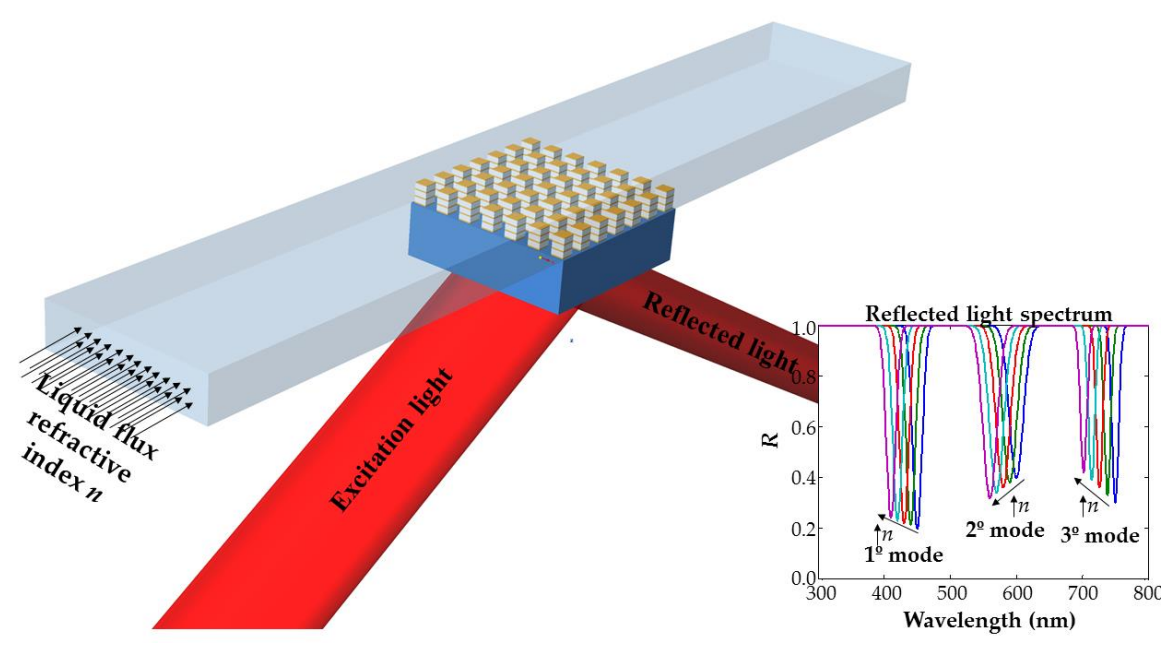

Figure 6.2. NPHM for sensing application. Changing the refractive index of the liquid flowing inside the microfluidic channels provoke a change in the NPHM resonant frequency, as shown in the figure inset. Note that the inset graphic is just an illustration about the sensor's behavior, and does not represent any simulated structure. 
CHAPTER 6 -

CONCLUSION AND PERSPECTIVES

to utilize the frequency shift and the resonance amplitude to infer the desired properties of the liquid above the NPHM. 


\section{Appendix A}

\section{A.1. Homogenization Procedure based on a Drude-Lorentz Dispersive Model for Metamaterials}

Metamaterials are well-known in the scientific community for presenting electromagnetic properties normally not found in conventional media, such as negative permittivity $(\varepsilon<$ 0 ) and/or negative permeability $(\mu<0)$ [1]. One way of obtaining such medium properties is with an array of split ring resonator cells with individual dimensions much smaller than the operating wavelength [12]. Due to the complex geometry of the resonators, the accurate extraction of electromagnetic parameters of this cell array is crucial for understanding its macroscopic behavior as a whole [24]. Several methods currently available in the literature obtain the properties of the medium using a formalism derived from scattering parameters [68], [79], [169].

A critical issue regarding parameter extraction procedures is that in certain frequency bands $\varepsilon$ and $\mu$ have no causal response $(\operatorname{Im}\{\varepsilon\}<0$ and/or $\operatorname{Im}\{\mu\}<0$ ) [88] (a behavior also observed in [24], [68], [79]). This issue can be circumvented with the adoption of transition layers as suggested in [88], thereby making the extracted parameters to have causal responses in the entire frequency band. However, the frequency dependence of the extracted $\varepsilon$ and $\mu$ vectors does not obey any known dispersive material model description. As a result, it becomes very time consuming to simulate the homogenized structure with a finite difference time domain (FDTD) method due to the requirement of temporal convolutions of $\varepsilon$ and $\mu$ [89], [90]. Fortunately, the computational performance can be improved if one approximates $\varepsilon$ and $\mu$ via Drude and Lorentz dispersive models, respectively [64], [91], [92]. This approach not only allows the temporal convolutions to be solved analytically, it also produces causal homogenized media $(\operatorname{Im}\{\varepsilon\}>0$ and $\operatorname{Im}\{\mu\}$ $>0)$.

Although widely used for parameter extraction of metamaterials, the Drude and Lorentz models do not produce electromagnetic parameters compatible with the observed behavior of these media for frequencies near the Lorentz model resonance frequency [64], [91], [92].

In this chapter, an improved homogenization procedure is proposed where the actual metamaterial cells are homogenized with a periodic array of a Drude-Lorentz-type metamaterial (MT) layer sandwiched between two transition layers (TL). Differently from [88], the MT layer utilizes Drude model for $\varepsilon$ and Lorentz model for $\mu$, while the TL layers use Drude model for $\varepsilon$ and $\mu=1$. This approach allows the homogenized medium to behave precisely as the actual metamaterial cells, making it possible to simulate this medium accurately even with two-dimensional FDTD methods (therefore reducing the computational cost). This assumption considerably improves the scattering parameters approach, as will be demonstrated later on for a three-cells thick metamaterial slab. 
Furthermore, this method is published in the Microwave and Optics Technology Letters (MOTL) [93]. The copyrights permission is found in Annex 3 of this thesis.

\section{A.2 Methodology}

\section{A.2.1 Drude-Lorentz Model (DL)}

The electromagnetic parameters of the metamaterial cells can be described with the Drude model for $\varepsilon$ and with the Lorentz model for $\mu$ as follows,

$$
\begin{gathered}
\varepsilon_{D}=\varepsilon_{\infty}-\frac{f_{p e}^{2}}{f\left(\mathrm{j} \Gamma_{e}+f\right)^{\prime}} \\
\mu_{L}=\mu_{\infty}-\frac{\left(\mu_{\infty}-\mu_{z}\right) f_{p m}^{2}}{f_{p m}^{2}-\mathrm{j} \Gamma_{e} f-f^{2}},
\end{gathered}
$$

where $\varepsilon_{\infty}$ and $\mu_{\infty}$ are, respectively, $\varepsilon_{D}$ and $\mu_{L}$ for $f \rightarrow \infty, \mu_{z}$ is $\mu_{L}$ for $f \rightarrow 0, f_{p e}$ is the electric plasma frequency, $f_{p m}$ is the magnetic resonance frequency, and $\Gamma_{e}$ and $\Gamma_{m}$ are, respectively, the electric and magnetic damping factors (related to the electron and whose values are always positive to preserve causality [68]). The adopted homogenization scheme is shown in Figure A.1, with (a) representing the original three cells thick metamaterial medium and (b) the homogenized medium. The choice of a three cells thick medium is solely due to computational limitations, as the proposed method is capable of handling any number of cells.

First, the extraction procedure requires the S-parameters $\left(S_{11}^{e}\right.$ and $\left.S_{21}^{e}\right)$ of the actual metamaterial cells (see Figure A.1 (a)) to be obtained, which can be carried out with the

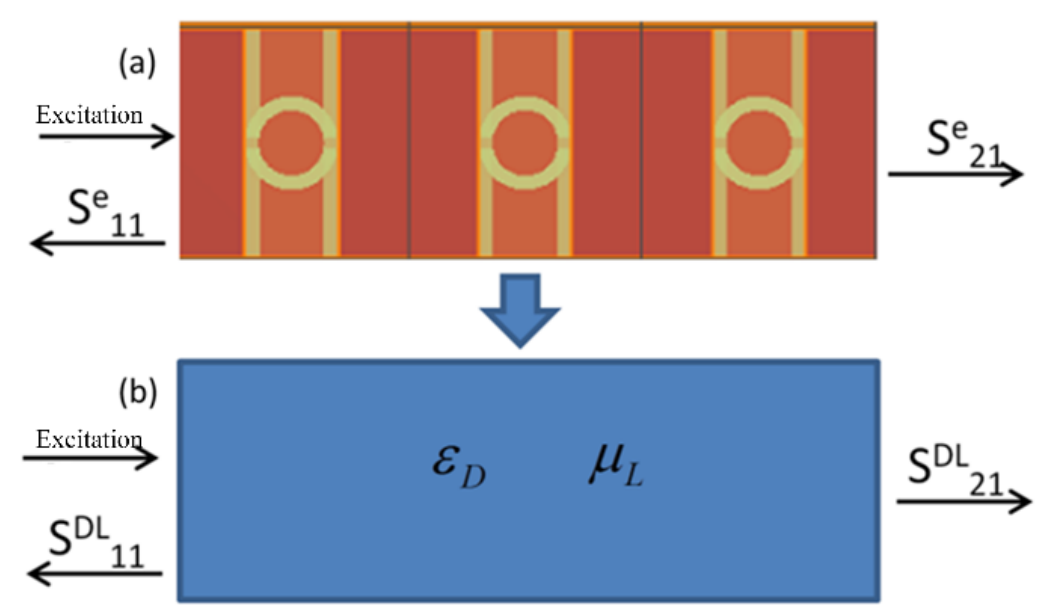

Figure A.1. Homogenization scheme based on the Drude-Lorentz model. (a) shows the original metamaterial cells to be homogenized while (b) shows the homogenized medium. 
commercial software High Frequency Structure Simulator (HFSS) [86], among others. Once the S-parameters are obtained, one can use the routine suggested in [68] to calculate the corresponding permittivity $\left(\varepsilon^{e}\right)$ and permeability $\left(\mu^{e}\right)$ of the metamaterial slab. The next step consists in finding an equivalent homogenized slab medium with Drude-type permittivity $\left(\varepsilon_{D}\right)$ and Lorentz-type permeability $\left(\mu_{\mathrm{L}}\right)$ that produce the same set of electromagnetic parameters as those of the original structure in Figure A.1 (a), namely, $\varepsilon^{e}$ and $\mu^{e}$. This method (DL) is here forth referred to as the conventional method. Ideally, $\varepsilon_{D}$ and $\mu_{\mathrm{L}}$ should be an exact match to $\varepsilon^{e}$ and $\mu^{e}$, respectively. However, there is always an error associated with the optimization procedure. This function adjusts the values of the variables in A.1 and A.2 up to a certain error $e_{D L}$ (relative to the DL method) defined as:

$$
e_{D L}=\frac{\sum_{m=1}^{N}\left(\frac{\left|\mu^{e}(m)-\mu_{L}(m)\right|}{\left|\mu^{e}(m)\right|}+\frac{\left|\varepsilon^{e}(m)-\varepsilon_{D}(m)\right|}{\left|\varepsilon^{e}(m)\right|}\right)}{2 N}
$$

The above equation represents the permittivity and permeability relative average error across the entire frequency range. Both DL S-parameters $\left(S_{11}^{D L}\right.$ and $\left.S_{11}^{D L}\right)$ are frequency dependent and defined as a vector of size $N$, where each element $(m)$ represents one frequency component.

\section{A.2.2 Transition Layer Method (TL)}

The homogenization scheme based on transition layers is shown in Figure A.2, where a slab consisting of $p$ MT layers requires $q$ TL layers, with $q=p+1$. Thus, each omega cell in Figure A.1 (a) is represented by a MT layer flanked by two TL layers. Accordingly, one MT layer and one TL layer must be added for each additional omega cell. Hence, the total length $d$ of the homogenized structure must equal the sum of the individual thicknesses of all omega cells in Figure A.1 (a). Moreover, it is necessary that the slab be symmetric in order to maintain the relation $S_{11}=S_{22}$ and $S_{21}=S_{12}$, which imposes the following constraint on the $i$-th layer: $\mathrm{MT}^{i}=\mathrm{MT}^{p-i+1}, \mathrm{TL}^{i}=\mathrm{TL}^{q-i+1}$ and $d_{i}=d_{p+q-i+1}$.

The relative permeability $\left(\mu_{M T}^{i}\right)$ and relative permittivity $\left(\varepsilon_{M T}^{i}\right)$ of the $\mathrm{MT}^{i}$ layer (with $i=1,2, . . p$ ) are defined via Drude (Figure A.1) and Lorentz (A.2) models, respectively. On

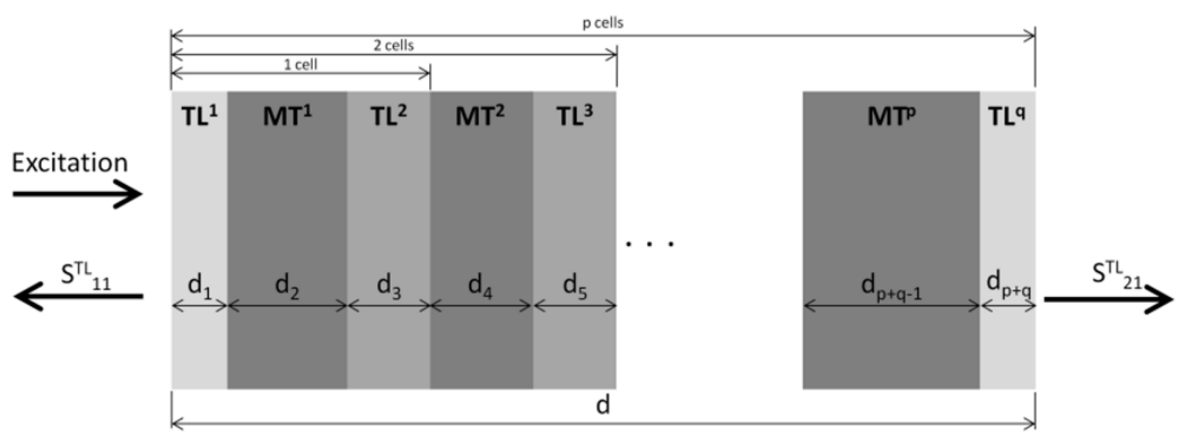

Figure A.2. Homogenization scheme of metamaterial cells with the TL model 
the other hand, in the transition layers $\operatorname{TL}^{m}(m=1,2, . . q)$, the permittivity $\left(\varepsilon_{T L}^{m}\right)$ is defined via Drude model (A.1) while the relative permeability is that of the vacuum $\left(\mu_{T L}^{m}=1\right)$.

Using the transfer matrix technique (TMT) it is possible to calculate the scattering parameters of a slab with $2 p+1$ layers, shown in Figure A.2, with a simple matrix operation for each layer [68] (this procedure works for both TE and TM polarization at normal incidence). The total transfer matrix $\left(T_{T}\right)$ is the product of the transfer matrix of each individual layer $\left(\boldsymbol{T}_{\boldsymbol{M T}}^{\boldsymbol{i}}\right.$ refers to layer $\mathrm{MT}^{i}$ and $\boldsymbol{T}_{\boldsymbol{T L}}^{\boldsymbol{m}}$ refers to layer $\left.\mathrm{TL}^{m}\right)$, such that:

$$
\begin{gathered}
\mathbf{T}_{\mathbf{T}}=\left[\begin{array}{ll}
T_{11} & T_{21} \\
T_{12} & T_{22}
\end{array}\right]=\mathbf{T}_{\mathrm{TL}}^{\mathbf{1}} \prod_{i=1}^{p} \mathbf{T}_{\mathrm{MT}}^{\mathbf{i}} \mathbf{T}_{\mathrm{TL}}^{\mathbf{i}+1},\left\{\begin{array}{l}
\mathbf{T}_{\mathrm{MT}}^{\mathbf{i}}=\mathbf{T}_{\mathrm{MT}}^{\mathbf{p}-\mathbf{i}+1} \\
\mathbf{T}_{\mathrm{MT}}^{\mathbf{i}+1}=\mathbf{T}_{\mathrm{MT}}^{\mathbf{p}-\mathbf{i}+2}
\end{array}\right. \\
\mathbf{T}_{\mathbf{M T}}^{\mathbf{i}}=\left[\begin{array}{cc}
\cos \left(k_{0} d_{2 i} \sqrt{\varepsilon_{M T}^{i} \mu_{M T}^{i}}\right) & -k_{0} \sqrt{\frac{\varepsilon_{M T}^{i}}{\mu_{M T}^{i}} \sin \left(k_{0} d_{2 i} \sqrt{\varepsilon_{M T}^{i} \mu_{M T}^{i}}\right)} \\
k_{0} \sqrt{\frac{\varepsilon_{M T}^{i}}{\mu_{M T}^{i}} \sin \left(k_{0} d_{2 i} \sqrt{\varepsilon_{M T}^{i} \mu_{M T}^{i}}\right)} & \cos \left(k_{0} d_{2 i} \sqrt{\varepsilon_{M T}^{i} \mu_{M T}^{i}}\right)
\end{array}\right] \\
\mathbf{T}_{\mathrm{TL}}^{\mathbf{i}}=\left[\begin{array}{cc}
\cos \left(k_{0} d_{2 m-1} \sqrt{\varepsilon_{M T}^{m}}\right) & -k_{0} \sqrt{\varepsilon_{M T}^{m}} \sin \left(k_{0} d_{2 m-1} \sqrt{\varepsilon_{M T}^{m}}\right) \\
k_{0} \sqrt{\varepsilon_{M T}^{m}} \sin \left(k_{0} d_{2 m-1} \sqrt{\varepsilon_{M T}^{m}}\right. & \cos \left(k_{0} d_{2 m-1} \sqrt{\varepsilon_{M T}^{m}}\right)
\end{array}\right]
\end{gathered}
$$

Hence, from the transfer matrix coefficients $\left(\boldsymbol{T}_{T}\right)$, it is possible to calculate the scattering parameters $\left(S_{11}^{T L}\right.$ and $\left.S_{21}^{T L}\right)$ of the multilayer slab [68]:

$$
\begin{aligned}
& S_{21}^{T L}=S_{11}^{T L}=\frac{1}{T_{11}+\frac{1}{2}\left(j k_{0} T_{12}+\frac{T_{21}}{j k_{0}}\right)}, \\
& S_{11}^{T L}=S_{22}^{T L}=\frac{\frac{1}{2}\left(j k_{0} T_{21}-\frac{T_{12}}{j k_{0}}\right)}{T_{22}+\frac{1}{2}\left(j k_{0} T_{12}+\frac{T_{21}}{j k_{0}}\right)}
\end{aligned}
$$

where the superscript TL refers to the proposed transition layer method. Once the homogenization procedure is completed, the next step is to calculate all coefficients in equations A.1 and A.2, as well as the thickness of each layer. The total number of variables is $4[(p+1) / 2\rceil+7\lfloor(p+1) / 2\rfloor$, which is higher than that of the DL method, therefore resulting in higher computational cost as well. However, this limitation is fully compensated by the increased accuracy of the proposed method. Once the scattering parameters $\left(S_{11}^{T L}\right.$ and $\left.S_{21}^{T L}\right)$ of the homogenized medium are obtained, the error of the TL method $\left(e_{T L}\right)$ can be calculated with the help of equation A.3, by simply replacing $e_{D L}$ by 
$e_{T L}, \varepsilon^{D L}$ and $\mu^{D L}$ by $\varepsilon^{T L}$ and $\mu^{T L}$, where $\varepsilon^{T L}$ and $\mu^{T L}$ are the permittivity and permeability extracted from $S_{11}^{T L}$ and $S_{21}^{T L}$ using the method described in [63].

\section{A.3. Results}

There are a number of different cell geometries in the literature that allow negative values of refractive index in a given frequency band [68], [70]. The omega cell [41] has been adopted here for convenience sake only. The cell geometry, along with pertinent geometric parameters, is shown in Figure A.3. Note that the excitation source must have its magnetic field $(\mathbf{H})$ oriented normal to the substrate surface ( $y$-axis) and its electric field $(\overrightarrow{\mathrm{E}})$ oriented parallel to the omega cell arms (z-axis). This source setup configuration guarantees negative refraction [41]. The present study considers three metamaterial cells evenly distributed along the direction of wave propagation ( $x$-axis). The substrate is alumina $\left(\varepsilon_{r}=9.2+0.012 j\right)$, and the cell is designed to resonate in the X-band ( $8 \mathrm{GHz}$ to 12 $\mathrm{GHz}$ ). The cell is surrounded by a vacuum box (hidden in Figure A.3) with periodicity of 5 - $\mathrm{mm}$ in the $y$-axis and 5 - $\mathrm{mm}$ in the $z$-axis.

The homogenization procedure is now carried out using both the conventional (DL) and the proposed (TL) methods. Note that the scattering parameters obtained with HFSS are used by the two methods. The convergence of this procedure can be accelerated if one notice in Figure A.4 that the cell presents a resonance at approximately $10.6 \mathrm{GHz}$. At this frequency point $S_{21}$ (obtained via HFSS) gets closer to zero, meaning that $f_{p m}=10.6 \mathrm{GHz}$ can be set as an initial guess. The initial guess for other variables can be seen Table A.1. The tolerance is set to $0.015(1.5 \%)$ with a maximum of 100,000 iterations. The frequency range is from $10.4 \mathrm{GHz}$ to $11.4 \mathrm{GHz}$ with $N=500$ discretization points. The optimized parameters that best approximate the electromagnetic properties of the analyzed medium are listed in Table A.

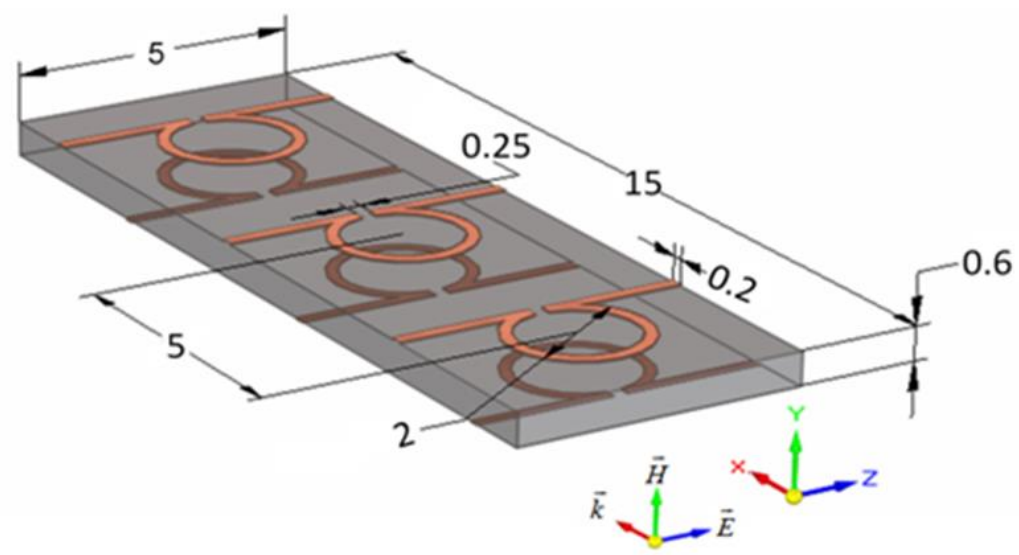

Figure A.3. Diagram of the unit cell of a three cells thick metamaterial slab. Periodic boundary conditions are used at $y= \pm 2.5 \mathrm{~mm}$ and $\mathrm{z}= \pm 2.5 \mathrm{~mm}$. Dimensions are in $\mathrm{mm}$. 
Table A.1. Initial Values for the Approximation Method.

\begin{tabular}{ccccccccr}
\hline \hline & $\varepsilon_{\infty}$ & $f_{p e}(\mathrm{GHz})$ & $\Gamma_{e}(\mathrm{MHz})$ & $\mu_{\infty}$ & $\mu_{z}$ & $f_{p m}(\mathrm{GHz})$ & $\Gamma_{m}(\mathrm{MHz})$ & $d^{i}(\mathrm{mr}$ \\
\hline $\mathrm{TL}^{1}$ & 1.75 & 15.00 & 100.0 & -- & -- & -- & -- & 0.5( \\
$\mathrm{MT}^{1}$ & 1.75 & 15.00 & 100.0 & 1.0 & 1.30 & 10.60 & 100.0 & $4.3 z$ \\
$\mathrm{TL}^{2}$ & 1.75 & 15.00 & 100.0 & -- & -- & -- & -- & 0.5( \\
$\mathrm{MT}^{2}$ & 1.75 & 15.00 & 100.0 & 1.0 & 1.30 & 10.60 & 100.0 & $4.3 z$ \\
$\mathrm{DL}$ & 1.0 & 15.00 & 100.0 & 1.0 & 1.20 & 10.60 & 100.0 & $15 .($ \\
\hline \hline
\end{tabular}

Table A.2. Homogenization parameters for the three cells thick metamaterial slab shown in Figure A.1 (a).

\begin{tabular}{ccccccccc}
\hline \hline & $\varepsilon_{\infty}$ & $f_{p e}(\mathrm{GHz})$ & $\Gamma_{e}(\mathrm{MHz})$ & $\mu_{\infty}$ & $\mu_{z}$ & $f_{p m}(\mathrm{GHz})$ & $\Gamma_{m}(\mathrm{MHz})$ & $d^{i}(\mathrm{~mm})$ \\
\hline $\mathrm{TL}^{1}$ & 1.70 & 21.85 & 47.13 & -- & -- & -- & -- & 0.26 \\
$\mathrm{MT}^{1}$ & 2.20 & 15.62 & 43.34 & 1.17 & 1.46 & 10.55 & 70.07 & 4.64 \\
$\mathrm{TL}^{2}$ & 1.16 & 29.10 & 104.8 & -- & -- & -- & -- & 0.28 \\
$\mathrm{MT}^{2}$ & 4.91 & 22.78 & 51.75 & 0.59 & 0.73 & 10.56 & 63.36 & 4.64 \\
$\mathrm{DL}$ & 2.75 & 18.25 & 54.08 & 1.05 & 1.26 & 10.62 & 71.30 & 15.0 \\
\hline \hline
\end{tabular}

With the data from Table A.2 it is possible to calculate the scattering parameters produced

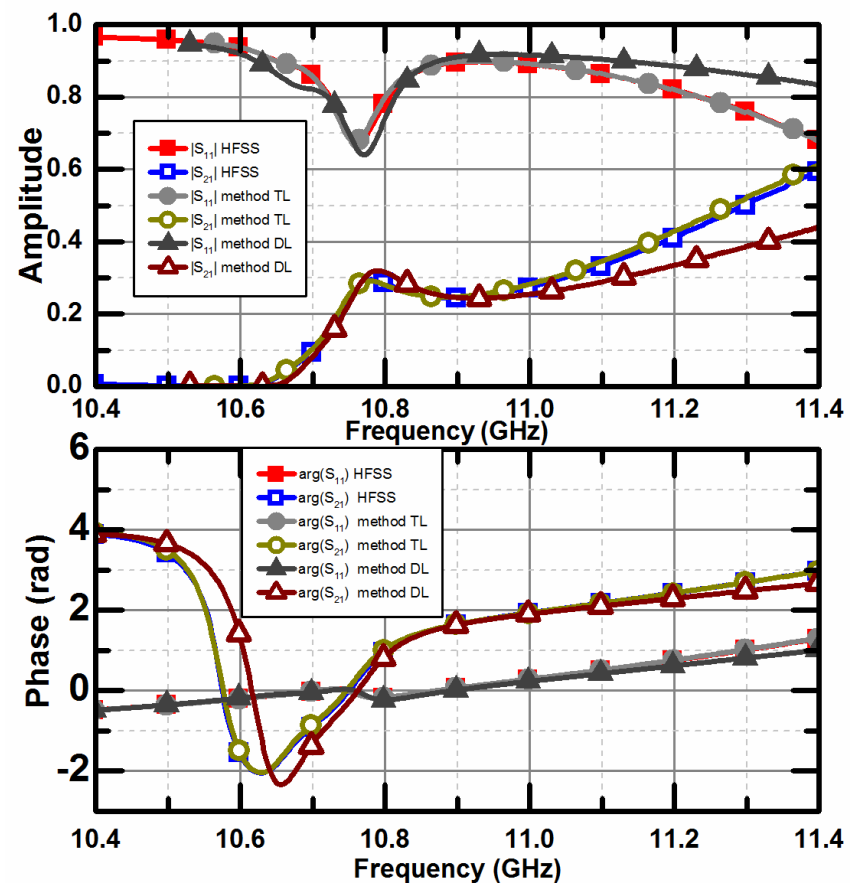

Figure A.4. Scattering parameters for the three cells thick metamaterial slab shown in Figure A.2 (a). The solid symbols represent $S_{11}$ and the hollow symbols $S_{21}$. Scattering parameters for the three cells thick metamaterial slab shown in Fig. 3. The solid symbols represent $S_{11}$ and the hollow symbols $S_{21}$. Squares represent parameters extracted with HFSS (benchmark), circles with the (proposed) TL method, and triangles with the DL method. 


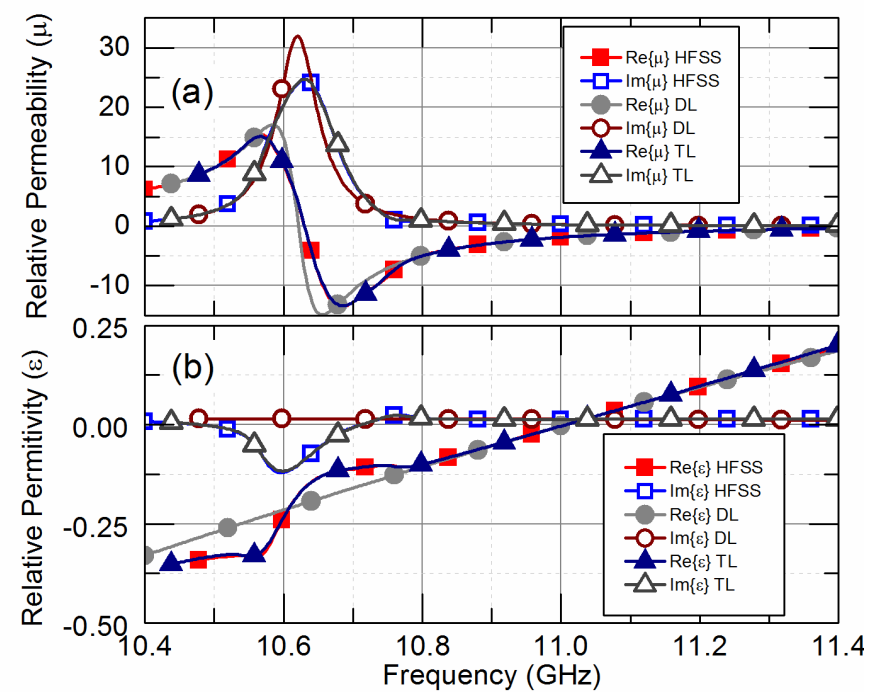

Figure A.5. Real (solid symbols) and imaginary (hollow symbols) parts of the permeability (a) and permittivity (b) for a three cells thick metamaterial slab obtained with HFSS (squares), DL method (circles), and TL method (triangles).

by the DL and TL extraction methods, which are plotted in Figure A.4. Observe that the TL method produces a more accurate representation of the curve extracted via HFSS (assumed here as benchmark) when compared to the DL method. The permittivity and permeability for the three cases (HFSS, TL and DL) are calculated using the parameter retrieval technique described in [68]. The real and imaginary parts of the permeability are shown in Figure A.5 (a), while the real and imaginary parts of the permittivity are shown in Figure A.5 (b). Differently from the DL method, the real and imaginary parts of both the permittivity and permeability obtained with the proposed TL method closely match those obtained with the HFSS. This is particularly true for the permittivity near the resonance frequency of $10.62 \mathrm{GHz}$, where $\varepsilon$ presents a resonance difficult to reproduce with the Drude model. A similar behavior is observed for $\mu$, which presents an asymmetry around the Lorentz resonance.

The relative permittivity and permeability error for both the DL and the TL methods is shown in Figure, A.6. It is noteworthy the error magnitude for the DL method around the resonance at $10.6 \mathrm{GHz}$, approaching $50 \%$. For frequencies above $10.8 \mathrm{GHz}$ the error magnitude decreases significantly, as observed in the inset in Figure, A.6. The TL method, by its turn, is not affected by the material models' resonances, resulting in an average relative error around $1.25 \%$ for the entire frequency range.

\section{A.4. Conclusions}

In this appendix we have demonstrated an improved homogenization procedure for metamaterial cells of arbitrary geometry. Differently from previous methods (that approximate $\mu$ and $\varepsilon$ with known dispersive models such as Lorentz and Drude, for 


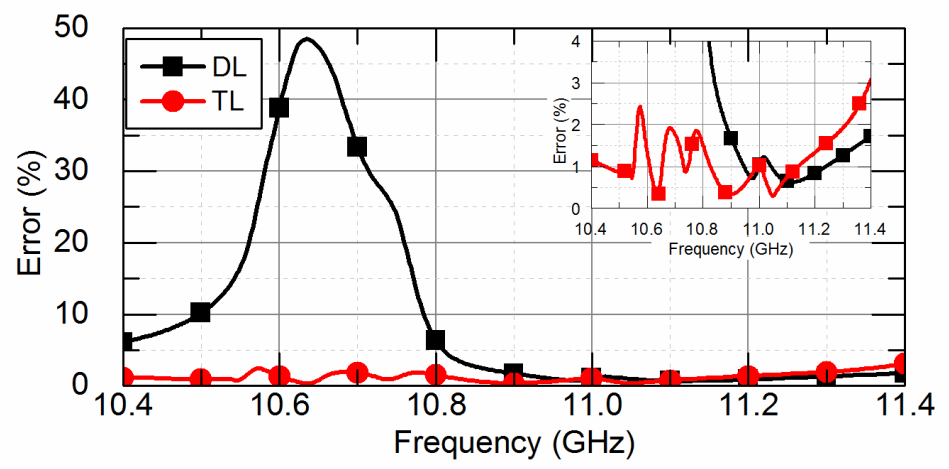

Figure A.6. Relative error for the DL (squares) and TL (circles) methods. The DL error peaks at the material resonance frequency. The inset highlights the average error of the proposed method.

example), the proposed method adopts Drude (for $\varepsilon$ ) and Lorentz (for $\mu$ ) models not only in the transition layers but also in the adjacent layers, considerably improving the scattering parameters approach. The validation procedure was carried out for a metamaterial slab with a thickness of three omega-shaped cells. Numerical results have shown that the error in the extracted parameters can be as low as 1.25\% (assuming HFSS as benchmark), a significant improvement if compared to conventional methods where errors as high as $10.1 \%$ can occur. Another major advantage inherent to the proposed technique is the possibility of reducing a 3D geometry to only two dimensions with high precision, resulting in significant computational gain (particularly in FDTD methods). 


\section{Appendix B}

Thinking in corroborating the approach proposed in chapter 3 of this thesis, the $\mathrm{PhD}$ candidate spent the year of 2017 at the VUB under the supervision of Profs. Heidi Ottevaere and Wendy Meulebroeck. During this period, we have fully characterized the emission spectrum and the lifetime of Cy7. Moreover, we also have characterized the process to mix the PMMA layer with dichloromethane to control the thickness of the PMMA layer. Later, we have hired a foundry to fabricate the HMM proposed on Figure 3.2 , but during the characterization of the structure we found out that the fabrication process had not been performed as expected. Consequently, we could not perform the Cy7 lifetime measurements on the top of the HMM.

In this sense, appendix $B$ describe the experimental results obtained during the period that the candidate spent at VUB. Subsection B.1 describes the process to mix the PMMA layer with dichloromethane to control the thickness of the PMMA layer. Subsection B.2 shows the experimental setup used to characterize the emission spectrum of the Cy7, and the measurements are shown in the subsection B.3. Then, subsection B.4 treats the measurements of the Cy7 lifetime. As mentioned, we have hired a foundry to fabricate the HMM proposed on Figure 3.2, but the fabrication process was not performed as expected (of the foundry responsibility). Subsection B.5 discuss the measurements results which lead us to the decision to not use the fabricated HMM.

\section{B.1. Polymer deposition}

As previously mentioned, the PMMA layer is deposited on the top of the HMM via spincoat technique. According Microchem 950PMMA A Resists A2 datasheet, it is possible to control $h$ by the right choice of the spin-coat velocity. The minimum (maximum) possible $h$ achieved by this technique is $60 \mathrm{~nm}(100 \mathrm{~nm})$, which is accomplished by setting the spincoat velocity to $5000 \mathrm{rpm}$ (1000 rpm). Nevertheless, as seen in chapter 3, HMM does not have a significant contribution on increasing $\Gamma$ of Cy7 for $h=60 \mathrm{~nm}$. Since $P_{\alpha}$ is higher close to the HMM surface, one approach to increase $P_{\text {eff }}$ is by reducing $h$ to values smaller than $60 \mathrm{~nm}$. This can be realized by mixing the PMMA with a solvent (such as ethanol or dichloromethane). The solvent's viscosity is lower than the PMMA's, thus by changing the PMMA's concentration and adjusting the spin-coat velocity, it is possible to achieve $h$ smaller than $60 \mathrm{~nm}$. The chosen solvent was dichloromethane, because it is a solvent for both PMMA and Cy7. The thickness of the film formed after spin-coating the solution with PMMA and dichloromethane is still unknown; thus, it is necessary to characterize this process. For charactering the film thickness achieved by the spin-coat process, we deposited the film on a microscope slide (MS) instead of the fabricated HMM, since they are cheaper. Normally, the MSs are saved for long periods, and during this time dust and greasy accumulate on their surface. Therefore, they need to go through a cleaning 


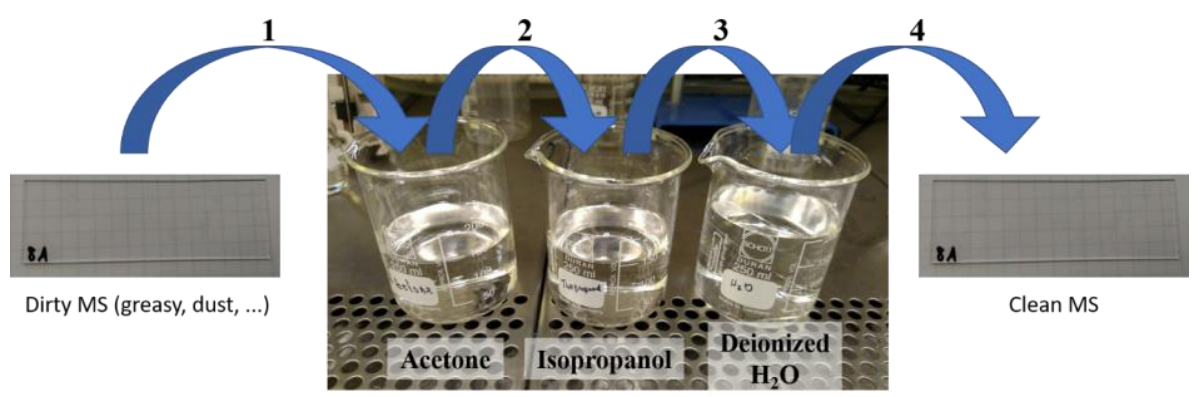

Figure B.1. Process to clean the MS.

processes before being used on the spin-coat machine. The four steps to clean the MS, depicted in Fig. B.1, are listed below:

1. Put the MS in the acetone for 5 minutes;

2. Transfer to the Isopropanol for 5 minutes;

3. Put the MS in the deionized water for 5 minutes;

4. Dry the MS with compressed air.

After cleaning the MS, the next step is to prepare the solution containing PMMA and dichloromethane. Note that for the thickness measurements and the calibration of the process, the solution was prepared without the Cy7. Furthermore, to access the repeatability of the procedure, 10 samples were prepared with this process. The spincoater machine and its parameters to prepare those samples are shown in Fig. B.2. The volumetric proportion of PMMA and dichloromethane is 1:1, resulting in a dichloromethane concentration $(C)$ of $50 \%$. The polymer films are prepared by spincoating $60 \mu \mathrm{l}$ of the PMMA+dicholoromethane solution on the MS (30 $\mu \mathrm{l}$ PMMA + $30 \mu \mathrm{l}$ dichloromethane). After spin-coating, the polymer solution is cured at 180 degrees for 2 minutes. Then, the thickness and roughness of the polymer films are measured by a stylus profilometer (Dektak 8) and the results are shown in Figure B.3 (a) and (b), respectively. The average thickness obtained with this procedure is $51.25 \mathrm{~nm}$, with an average error as low as $3.71 \mathrm{~nm}$. Based on the thickness and the low standard deviation, it is possible to decrease, with high reproducibility, the polymer film thickness using the dichloromethane. Furthermore, the average roughness of the samples is $2.99 \mathrm{~nm}$,

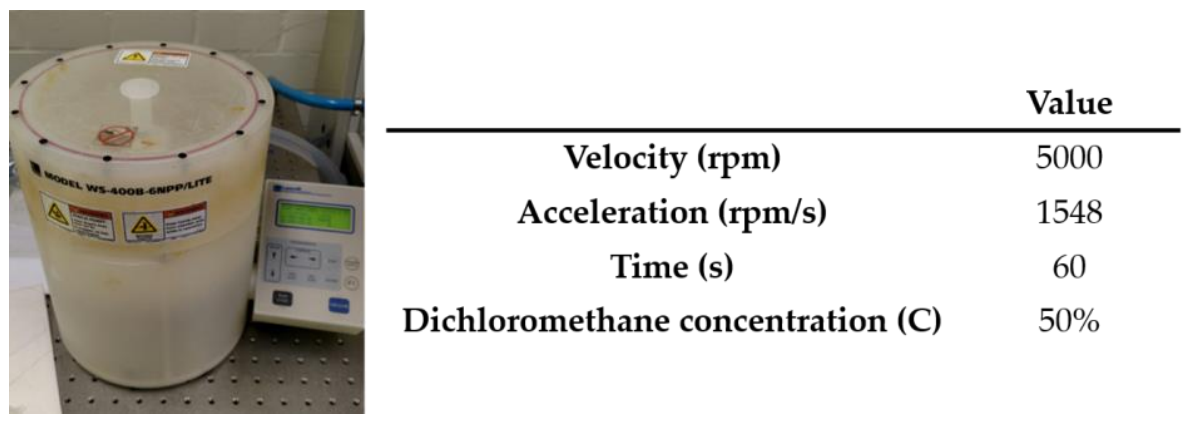

Figure B.2. The spin coater and the parameters utilized to deposit the PMMA layers on the top of the MS. 
(a)

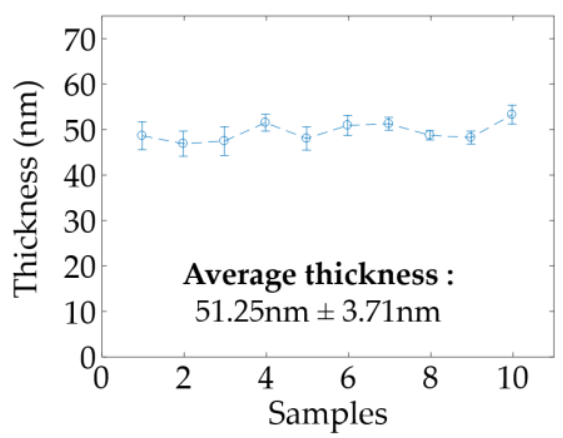

(b)

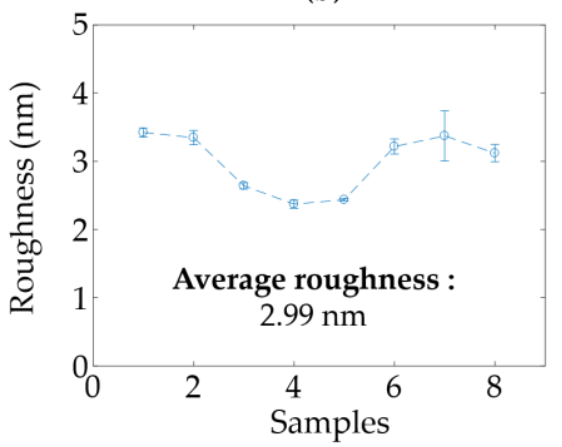

Figure B.3. Thickness (a) and roughness of 10 samples of PMMA and dichloromethane solution spin coated on top of a MS.

meaning that, in addition to have high confidence on the polymer thickness, its variation along the MS slide is very smooth.

After charactering the spin-coating procedure, the next step consists in controlling the thickness of the film. In this sense, we vary the dichloromethane concentration $(C)$ in the solution from $0 \%$ to $80 \%$, fabricating 3 samples per concentration. The solution is spincoated with the same volume $\left(\begin{array}{lll}6 & 0 \mu l\end{array}\right)$ and parameters (Figure B.2) as previously mentioned. The thickness measurements are performed via ellipsometry at $773 \mathrm{~nm}$, due to its higher precision compared with the profilometry technique. Furthermore, ellipsometry also provides the refractive index of the polymer $\left(n_{d}\right)$, which was used in all simulations performed on this thesis. Figure B.4 shows the thickness (a) and $n_{d}(\mathrm{~b})$ as function of the dichloromethane concentration. As can be seen, the low viscosity of the dichloromethane helps decreasing the thickness of the film. And by controlling its proportion in the solution, it is possible to adjust the film thickness from $80 \mathrm{~nm}(0 \%)$ to 39 $\mathrm{nm}(80 \%)$. Furthermore, the low error and linear behavior of the thickness measurements (squares) allows creating a fitting of the measurements with a straight line (solid line). This line allows to infer the polymer thickness knowing $C$, the dichloromethane

(a)

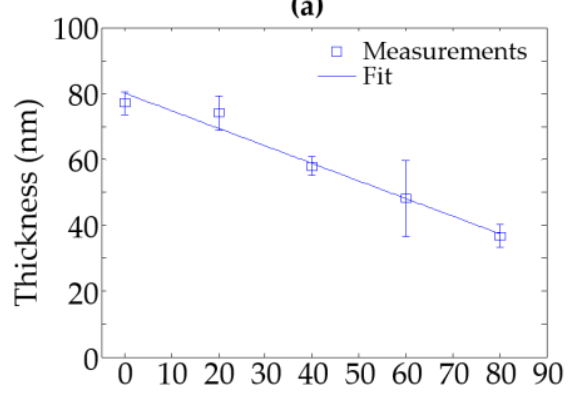

Dichloromethane concentration (\%)

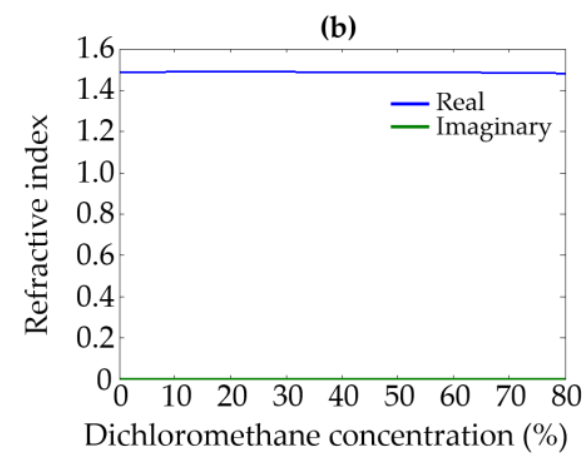

Figure B.4. Thickness (a) and roughness of 10 samples of the solution with PMMA and dichloromethane spin coated on the top of a MS. 


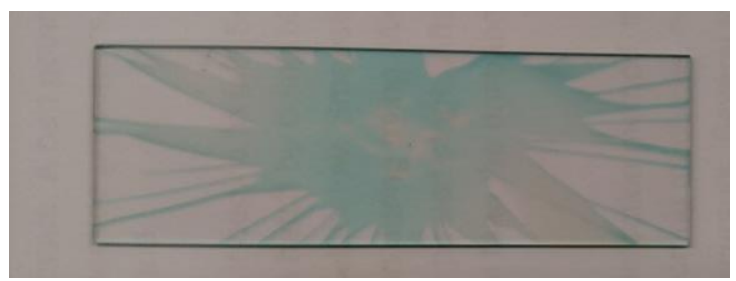

Figure B.5. Cy7 spin-coated on the top of the MS.

concentration. The real (blue) and imaginary (green) part of $n_{d}$ are shown in Figure B.4 (b). In contrast to the thickness, $n_{d}$ is almost constant with $C\left(n_{d} \cong 1.49\right)$ because of the similar refractive index of the solution elements (PMMA and dichloromethane). Thus, in this thesis, we consider $n_{d}=1.49$ as the polymer refractive index, regardless of $C$.

With the control of polymer thickness, the next step is to deposit the Cy7 molecules over the MS. To this end, $5 \mathrm{mg}$ of Cy7 is dissolved in $500 \mu \mathrm{l}$ of dichloromethane, resulting in the solution 1 (10 g/l of Cy7). Then, $30 \mu \mathrm{l}$ of solution 1 is mixed with $30 \mu \mathrm{l}$ of PMMA, resulting the solution 2 (5 g/l of Cy7), which is finally spin-coated on the top of the MS with the parameters shown in Figure B.2. According to Figure B.4, with a 50\% concentration, the estimate polymer thickness is $50 \mathrm{~nm}$. Figure B.5 shows the solution 2 after spin-coated over the MS. Although the spin-coating process did not spread the solution uniformly over MS surface, the central area presents regularity. Therefore this is the region chosen for the Cy7 experimental characterization, which is discussed in the next section.

\section{B.2. Experimental setup}

The initial step for the Cy7 spectrum and lifetime measurements is to prepare the experimental setup. As mentioned in subsection 3.3.2, it is possible to access the radiation parameters of perpendicularly or parallel polarized QE independently, due to their different maximum emission angles. Figure B.6 shows the radiation pattern for a perpendicular (thin blue line) and parallel (thin red line) QE. This figure also shows the best positions to place the sensor to measure the radiation for perpendicular (thick black line) and parallel (thick red line) QE. As can be seen, if the measurements of the radiation parameters of parallel QEs are of interest, we recommend the sensor to be placed at an
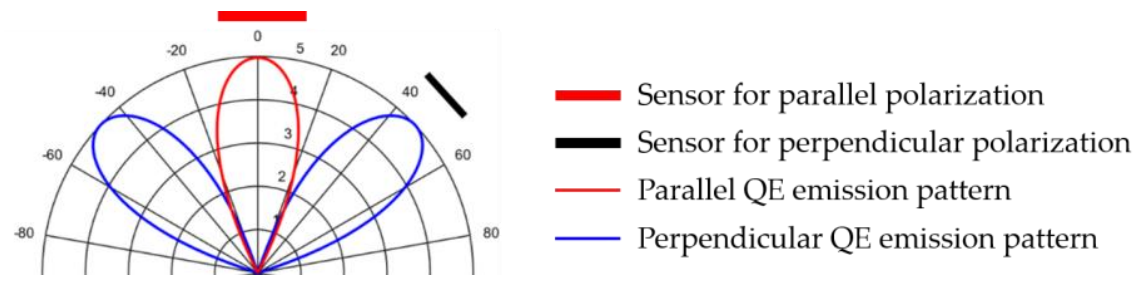

Figure B.6. Radiation pattern for a perpendicularly (blue) and parallel (red) polarized dipole. The figure also shows the best position to put the sensors to measure the power and lifetime of the Cy7 for each polarization. 
(a) - Parallel Cy7

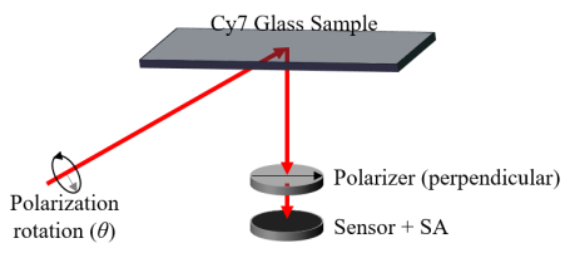

(b) - Perpendicular Cy 7

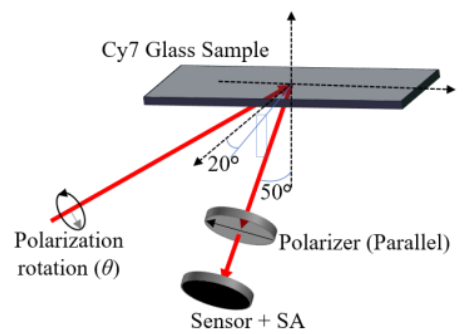

Figure B.7. Proposed setup to measure the lifetime of the Cy7 dyes for parallel (a) and perpendicular (b) polarization.

angle $\mathrm{o} 0^{\circ}$, because this is the angle where most of the waves are propagating. However, for the perpendicular polarization the sensor must be placed at an angle of $50^{\circ}$.

Furthermore, to control which QE polarization are being exited, we propose the setup illustrated in Figures B.7 (a) and (b) for perpendicular and parallel QE, respectively. For both setups, the excitation laser (at $\lambda=750 \mathrm{~nm}$ ) impinges almost tangentially with the Cy7 glass samples. This excitation configuration allows changing the orientation electric field that impinges on the samples from perpendicular to parallel by a simple rotation of the laser. This excitation spin allows controlling which QEs polarization are mostly excited. It is worth noticing that in real scenarios, the QEs are not strictly perpendicularly or parallelly polarized, they can have moment in both directions. Therefore, although the laser polarization helps prioritizing, it does not guarantee that only one polarization is excited. In order to enhancing the polarization control, the sensors are placed at different positions for perpendicular and parallel polarization, as depicted in Figures B.7 (a) and (b). To measure the radiation for parallel polarized dyes, the sensor is placed normal to the glass surface (Figure B.7 (a)), while for perpendicular dyes it is rotate by $50^{\circ}$ from the incident plane (Figure B.7 (b)). Furthermore, the perpendicular and parallel QEs radiate waves with different electric field orientation. Therefore, we also place a polarizer in order to reduce the cross polarization in the system. After the polarizer, the Cy7 emission is coupled into a fiber that is connected to a spectrum analyzer (SA) for the power measurements or to a photon counter for the lifetime measurements. The next section describes the spectrum measurements.

\section{B.3. Cy7 spectrum characterization}

Once the setups proposed in Figure B.7 (a) and (b) are built as shown in Figure B.8. we divided the setup in two parts, namely guiding the laser (Figure B.8 (a)) and the sample holder (Figure B.8 (a)). The pulsed laser available at VUB capable of generating light at $\lambda$ $=750 \mathrm{~nm}$ is the Tsunami Mode-Locked Ti:Sapphire Laser (Spectra Physics). Besides having a high-power output $(1.23 \mathrm{~W})$, this laser is fixed due to its size. For this reason, we had to create a mechanism using multiple mirrors to guide the light from the laser output to the sample, as seen in detail in Figure B.8 (b). Furthermore, Part 1 is also responsible for 
(a)
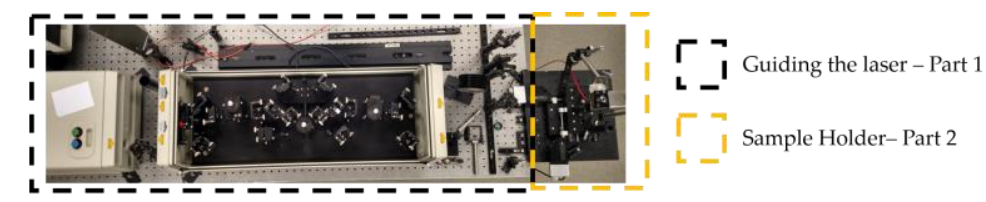

(b)

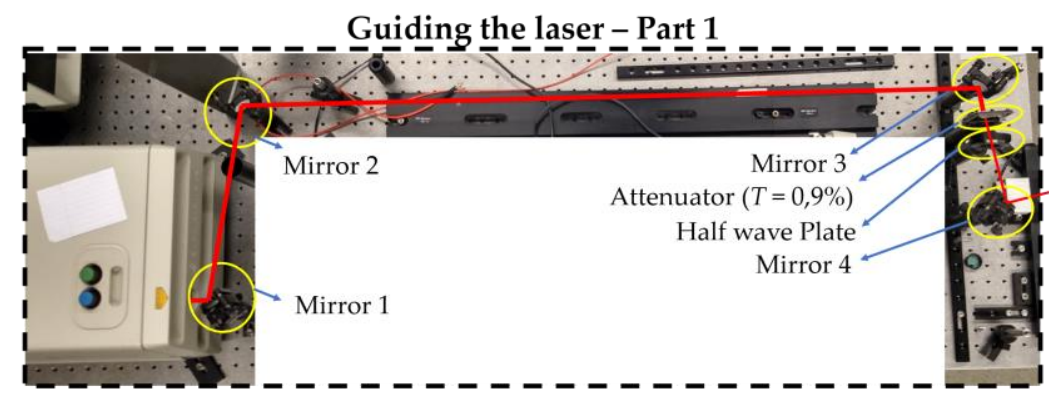

Sample Holder - Part 2

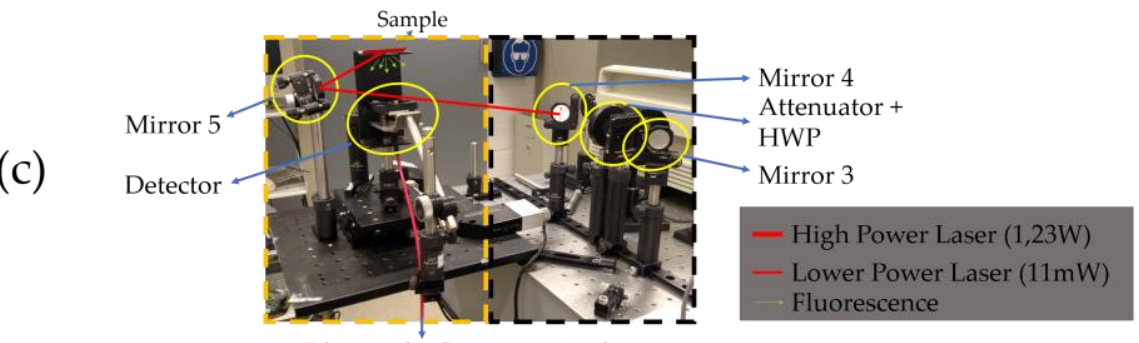

Fiber to the Spectrum Analyzer

Figure B.8. Proposed setup to measure the lifetime of the Cy7 dyes for parallel (a) and perpendicular (b) polarization.

controlling the laser polarization (using a half wave plate) and for attenuating the high power (using a $99.1 \%$ attenuator). After being guided, rotated and attenuated, the laser is conducted to the sample using aluminum mirrors, as seen in Figure B.8 (c). The Cy7 inside the samples excites at $\lambda=773 \mathrm{~nm}$, and its fluorescence emission is collected by a lens that couples the light into an optical fiber. The optical fiber is than connected to the optical spectrum analyzer (Avantes).

The first measurement consists in characterizing the spectrum of the excitation laser, which is accomplished by placing an empty MS (with no fluorescent molecules) at the sample position of the setup. Since there are no filters to block the excitation laser in the setup, knowing the laser's spectrum is crucial for removing its influence with the fluorescence measurements. After charactering the laser, the emission spectrum of Cy7 is then measured using the setup described in Figure B.8 and the results are compared to the datasheet. Figure B.9 shows the absorption (green solid line) and emission (blue solid line) spectrum of the Cy7 according to the datasheet. This figure also shows the Cy7 emission measured using the proposed setup as dashed blue line. Note in this curve that the power related to the excitation laser is already removed. Moreover, the measured curve is fitted by an exponential curve $\left(F l_{\text {fit }}\right)$, as follows, 


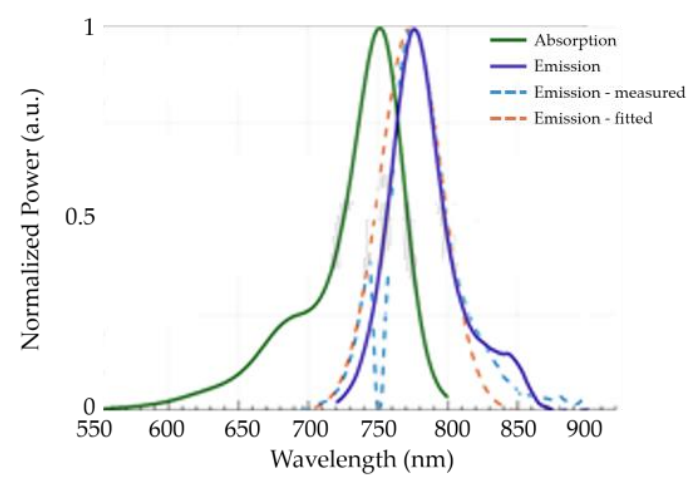

Figure B.9. Experimental (dashed line) and theoretical (solid line) emission spectrum of the Cy7. The experimental data was fitted using a Gaussian profile (red dashed line).

$$
F l_{f i t}(\lambda)=\exp \left(\left(\frac{\lambda-\lambda_{0}}{A}\right)^{2}\right)
$$

where the best fit parameters are $\lambda_{0}=774 \mathrm{~nm}$ and $A=1 \mathrm{e} 3$. According to (B.1), the measured emission wavelength is $774 \mathrm{~nm}$, which was close to the datasheet, $773 \mathrm{~nm}$. Also note in Figure B.9 that the experimental emission curve is close to the datasheet, corroborating the validity of our measurements.

\section{B.4. Lifetime measurements}

The next step of the $\mathrm{Cy} 7$ characterization consists in measuring its lifetime. Once again, we adopted the setup proposed on Figure B.8. Nevertheless, the optical fiber is now connected to a device that measures the QEs lifetime. This device was developed by Hans Ingelberts, Department of Electronics and Informatics at the Faculty of Engineering of the VUB. Figure B.10 shows the instrument reference function (IFR, blue), the measured decay (red) and the decay fit to eliminate the noise (black) for parallel (a) and perpendicular (b) polarized QEs. The fit is performed considering a single negative exponential decay, as:

$$
g(t)=\exp \left(\left(\frac{t}{\tau}\right)^{2}\right)
$$

where $\tau$ is the system lifetime. The best $\tau$ for perpendicular and parallel polarization are $269.5 \mathrm{~ns}$ and $213.5 \mathrm{~ns}$, respectively. Note that for these measurements, the Cy7 is embedded in a polymer with $n=1.49$ deposited on the top of a glass slide, and both of these parameters influence the lifetime of the system. In order to calculate the Cy7 lifetime in free space $\left(\tau_{0}\right)$, we calculate the theoretical $g(t)$ for the proposed situation and optimized the $\tau_{0}$ in order to achieve the best approximation for the theoretical and experimental 


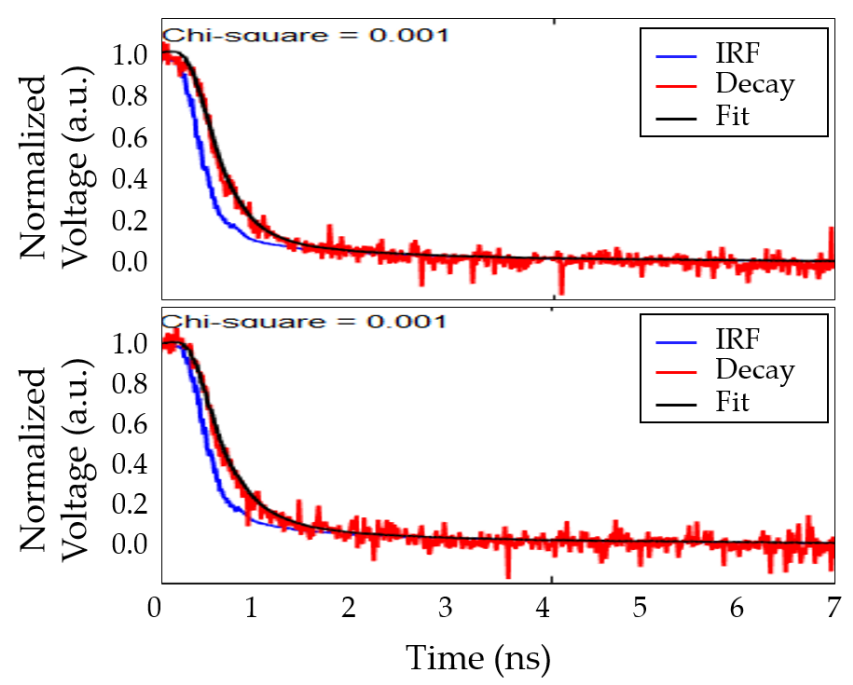

Figure B.10. Instrument reference function (IFR, blue), the measured decay (red) and the decay fit to eliminate the noise (black) for parallel (a) and perpendicular (b) polarized QEs.

decay curves. This process results in $\tau_{0}=350 \mathrm{ps}$. In this sense, Figure B.11 (a) shows $g(t)$ for the theoretical (considering $\tau_{0}=350 \mathrm{ps}$ ) perpendicular (solid blue) and parallel polarization (solid red). This graph also shows $g(t)$ for the measured perpendicular (dashed yellow) and parallel polarization (dashed purple), in addition to the free space decay curve as reference. As can be noticed by Figure B.11 (a), using $\tau_{0}=350 \mathrm{ps}$ is a good approximation for the $\mathrm{Cy} 7$, as the theoretical and experimental decay curves are close. Figure B.11 (b) shows the theoretical and measured lifetime reduction for both polarizations. Therefore, the measured Cy7 lifetime is 350 ps.

(a)

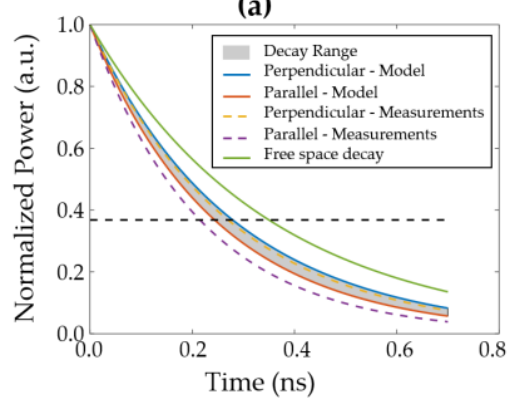

(b)

\begin{tabular}{ccc} 
& Theoretical & Measured \\
\hline $\boldsymbol{\tau}_{\perp} / \boldsymbol{\tau}_{\mathbf{0}}$ & 0.78 & 0.77 \\
$\boldsymbol{\tau}_{\|} / \boldsymbol{\tau}_{\mathbf{0}}$ & 0.70 & 0.61
\end{tabular}

Figure B.11. Experimental (dashed line) and theoretical (solid line) emission spectrum of the Cy7. The experimental data was fitted using a Gaussian profile (red dashed line). 


\section{B.5. Fabrication of HMM}

After fully characterizing the Cy7 fluorescence parameters, the next step would be fabricating the HMM shown in Figure 3.2. For this purpose, we hired a foundry. The fabricated structures were submitted to a scanning electron microscope (SEM) for analyzing the fabrication. Figures B.12 (a-d) show the SEM image of the top metallic layer with different scales (50 $\mu \mathrm{m}$ (a), $50 \mu \mathrm{m}$ (b), $10 \mu \mathrm{m}$ (c), $5 \mu \mathrm{m}$ (d)). As noticed from Figures B.12 $(a, b)$, the metallic top layer deposition is not uniform and present several regions where the metal is not correctly deposited (darker areas of the image). The presence of metallic clusters in the zoomed in regions (Figure B.12 (c,d)) indicates that the sputtering procedure was incorrectly performed. Consequently, the fabricated structure could not present the electromagnetic behavior expected from the designed HMM. In this sense, we did not perform any lifetime and emission spectrum measurements using the fabricated structure.
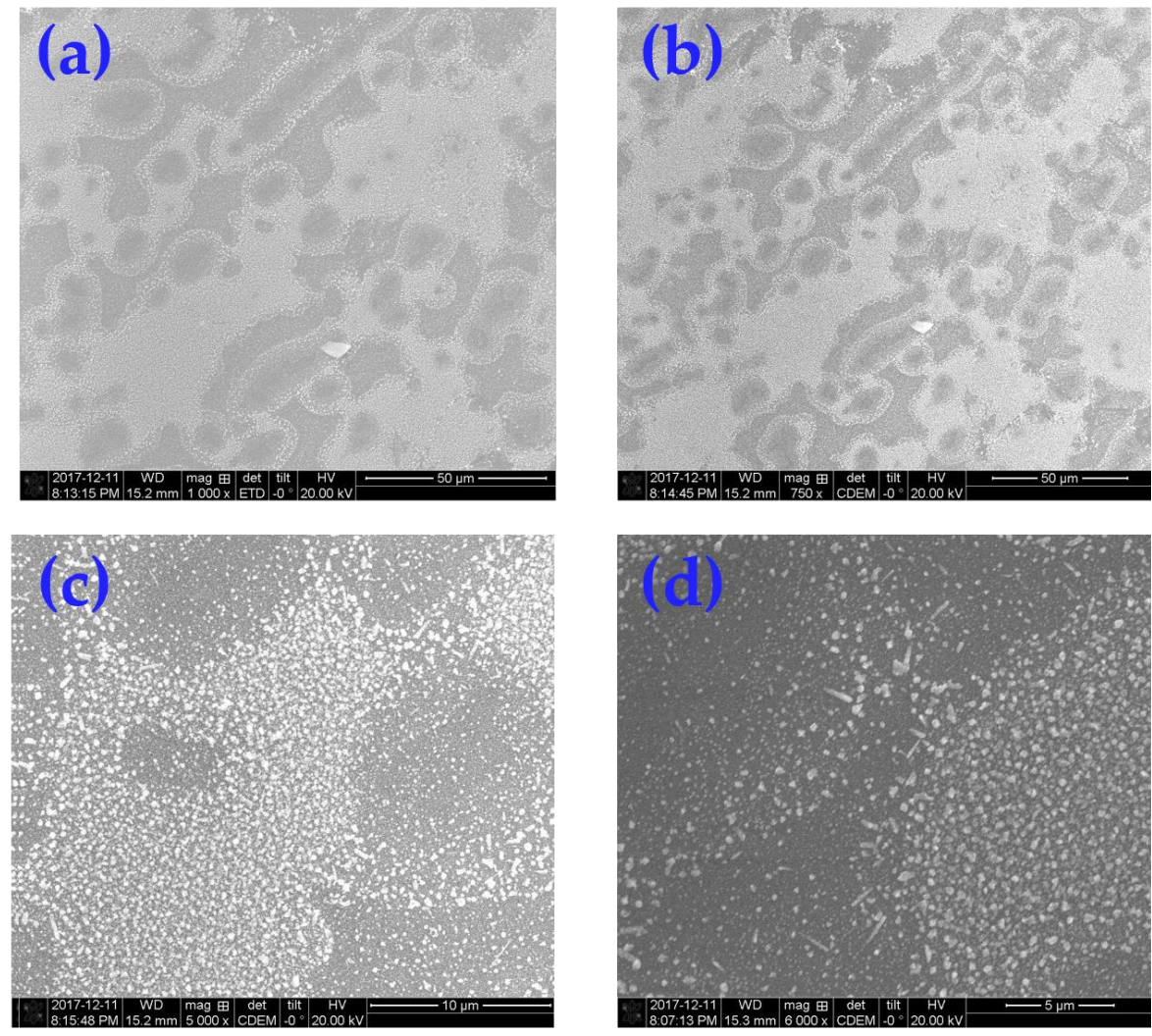

Figure B.12. (a-d) shows the pictures from the HMM surface taken by the SEM with different zooms (a) $50 \mu \mathrm{m}$, (b) $50 \mu \mathrm{m}$, (c) $10 \mu \mathrm{m}$ and (d) $5 \mu \mathrm{m}$. As can be seen, the HMM surface is not smooth and could not be used for lifetime measurements. 



\section{Appendix C}

\section{C.1. Lumerical 2D-FDTD Simulation}

The HMM with the metallic gratings on top were drown in Lumerical FDTD, as shown in Fig. A.3. The description of the simulation is listed below:

- $\quad$ Perfect Matched Layers (PML) for the surround the boundary condition.

- The total simulation area is $4 \mu \mathrm{m} \times 4 \mu \mathrm{m}$ and the mesh inside the structure is $\Delta x=\Delta y=0.5 \mathrm{~nm}$.

- Taking advantage of the symmetry of the simulations, we utilized the Asymmetric and Symmetric boundary condition for parallel or perpendicular dipole, respectively.

- A broadband (673-873nm) dipole source centered at $\lambda=773 \mathrm{~nm}$ was placed $10 \mathrm{~nm}$ under the HMM.

- Two vertical monitors were placed above the gratings in order to calculate the radiation pattern in the far field.

- By integrating the Poynting vector over the lines that surround the simulation area above the metallic gratings we calculated the power radiated to the far field.

- The calculation of the Purcell factor was performed by the Lumerical FDTD.
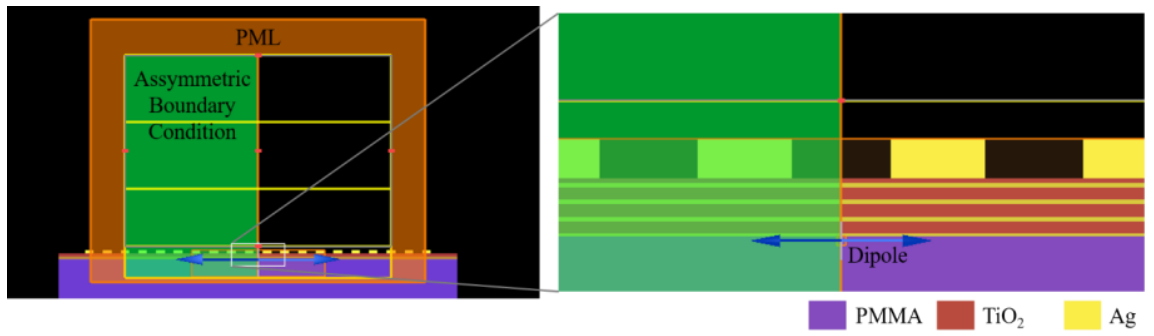

Figure C.1 Schematic of the Lumerical 2D-FDTD simulation. 



\section{List of Publications}

\section{Peer-reviewed Journal Articles}

1. A. F. Mota, A. Martins, V. Pepino, H. Ottevaere, W. Meulebroeck, F. L. Teixeira, B.-H. V. Borges, "Semianalytical modeling of quantum emitters embedded in nano-patterned hyperbolic metamaterials," submitted to the Journal of the Optical society of America $B$.

2. A. F. Mota, A. Martins, H. Ottevaere, W. Meulebroeck, E. R. Martins, J. Weiner, F. L. Teixeira, B.-H. V. Borges, "Semianalytical model for design and analysis of grating-assisted radiation emission of quantum emitters in hyperbolic metamaterials," ACS Photonics, vol. 5, no. 5, pp. 1951 - 1959, 2018.

3. A. F. Mota, A. Martins, J. Weiner, F. L. Teixeira, B.-H. V. Borges, “Constitutive parameters retrieval for uniaxial metamaterials with spatial dispersion," Physical Review B, vol. 94, no. 11, pp. 115410, 2016.

4. A. F. Mota, B.-H. V. Borges, "Improved homogenization procedure based on a Drude-Lorentz dispersive model for metamaterials," Microwave and Optical Technology Letters, vol. 58, no. 6, pp. 1490 - 1494, 2016.

\section{International Conferences Proceedings Articles}

1. A. F. Mota, A. Martins, V. Pepino, E. R. Martins, J. Weiner, F. L. Teixeira, B.-H. $\mathrm{V}$. Borges, "D semi-analytical model for optimizing the radiation emission of quantum emitters embedded in a bounded nano-patterned hyperbolic metamaterial," 12th International Congress on Artificial Materials for Novel Wave Phenomena - Metamaterials. Espoo, 2018.

\section{Publications not Related to this Thesis}

\section{Peer-reviewed Journal Articles}

1. V. Pepino, A. F. Mota, F. L. Teixeira, B.-H. V. Borges, "Fano-assisted tunable Xband microwave ring resonator," to be submitted to IEEE antennas and wireless propagations letters.

2. A. Martins, J. Li, A. F. Mota, V. Pepino, Y. Wang, L. G. Neto, F. L. Teixeira, E. R. Martins, B.-H. V. Borges, "Broadband c-Si metasurfaces with polarization control at visible wavelengths: applications to 3D stereoscopic holography," Optics express, vol. 26, no. 23, pp. 30740-30752, 2018. 
3. V. Pepino, A. F. Mota, A. Martins, B.-H. V. Borges, “3D printed dielectric metasurfaces for antenna gain improvement in the Ka-band," IEEE antennas and wireless propagations letters, 2018.

4. A. Martins, J. Li, A. F. Mota, Y. Wang, L. G. Neto, J. P. do Carmo, F. L. Teixeira, E. R. Martins, B.-H. V. Borges, "Highly efficient holograms based on c-Si metasurfaces in the visible range," Optics express, vol. 26, no. 8, pp. 9573-9583, 2018.

5. A. F. Mota, A. Martins, P. L. Bertarini, B.-H. V. Borges, "Dispersion management with microwave chirped pulses in metamaterials in the negative refraction regime," IEEE antennas and wireless propagation letters, vol. 14, pp. 1377 - 1380, 2015.

\section{International Conferences Proceedings Articles}

1. A. Martins, J. Li, A. F. Mota, Y. Wang, L. G. Neto, J. P. do Carmo, F. L. Teixeira, E. R. Martins, B.-H. V. Borges, “Crystalline Silicon (c-Si) Metasurface Holograms in the Visible Range," Digital Holography and Three-Dimensional Imaging Optical Society of America (OSA). Orlando, 2018.

2. V. Pepino, B. A. Francisco, A. F. Mota, B.-H. V. Borges, "Metamaterials in the microwave regime," II IWADA - International Workshop on Advanced Dielectrics and Applications. São Carlos, 2017. 


\section{List of Acronyms}

1D .One dimension

2D Two dimensions

2D-dipole Two-dimensions dipole

2D-FT Two-dimensions Fourier Transform

2D-SAM .Two dimensions semi-analytical model

3D Three dimensions

3D-SAM ..Three dimensions semi-analytical model

ABC Additional boundaries condition

Ag Silver

Au ..Gold

CAPES .Coordenação de aperfeiçoamento pessoa de ensino superior

$\mathrm{CNPq}$ .Conselho nacional de desenvolvimento científico e tecnológico

CPR. .Complete parameter retrieval

Cy7 Cyanine 7

DL .Drude-Lorentz model

DNG .Double negative medium

DSP. Double positive medium

EMT Effective medium theory

ENG Epsilon negative medium

ENZ. Epsilon near zero medium

FAPESP ..Fundação de amparo a pesquisa do estado de São Paulo

FDTD Frequency-domain time-discrete

FEM .Finite elements method

FT Fourier transform

FWHM .Full-width half maximum

HFSS .High frequency structure simulator

HMM ..Hyperbolic Metamaterials

HMM_ch3 .Hyperbolic Metamaterials proposed in chapter 3

HMM_opt Hyperbolic Metamaterials optimized in chapter 4 .Inverse Fourier transform 


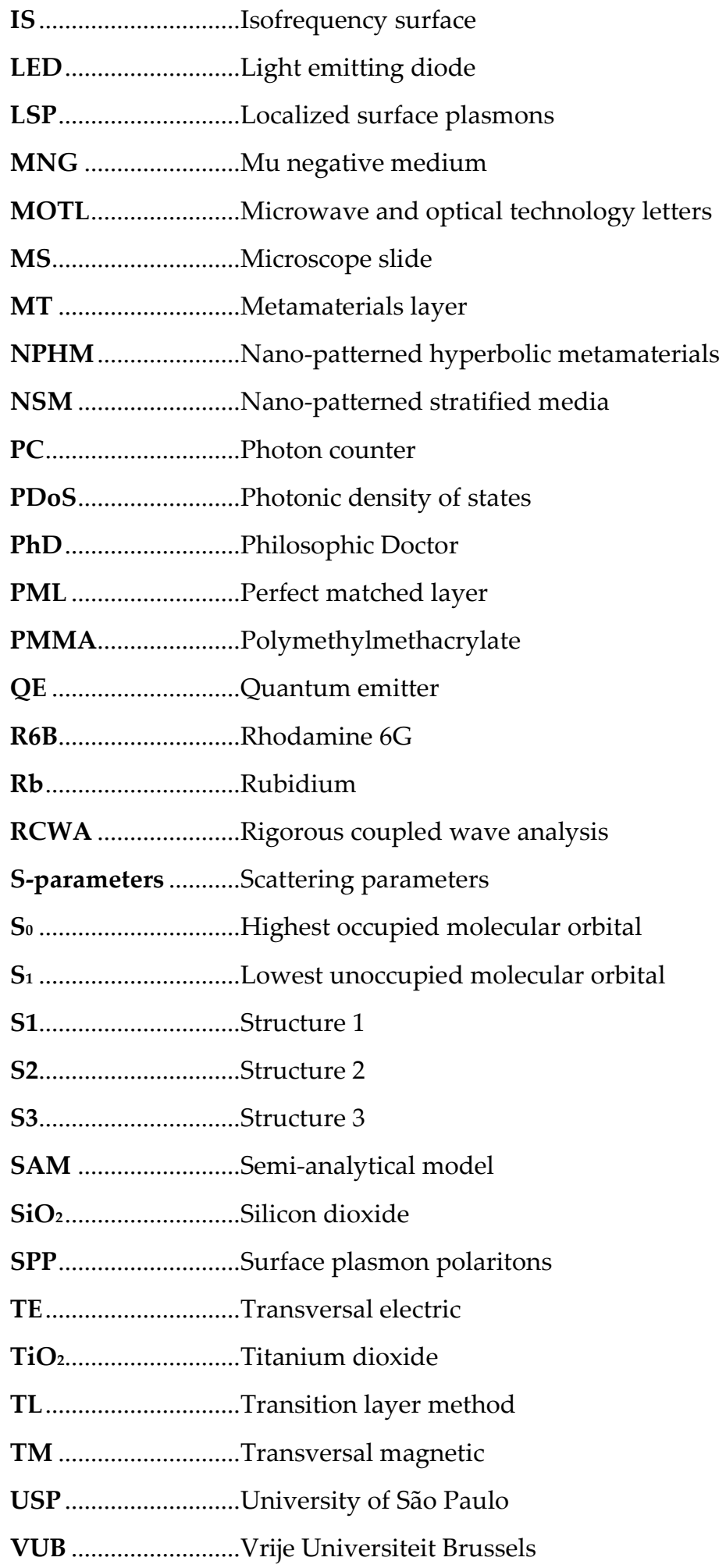




\section{List of Symbols}

\section{Latin - Roman letters}

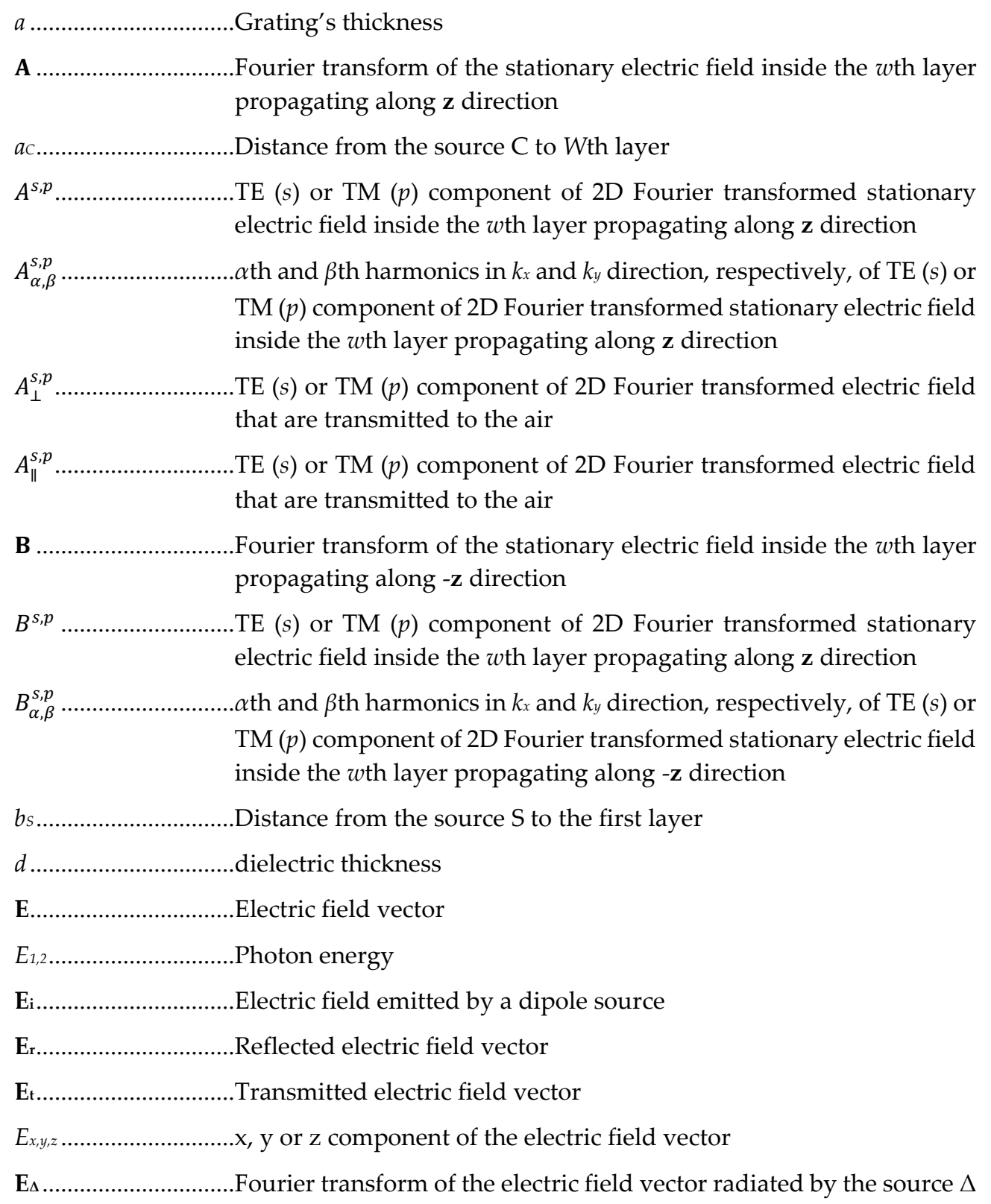


$\mathbf{E}_{\bar{\Delta} \mathbf{w}}$ ..Fourier transform of the electric field vector transmitted from the substrate $(\bar{\Delta}=\mathrm{S})$ or the cover $(\bar{\Delta}=\mathrm{C})$ to the $w$ th layer

$E_{\bar{\Delta} w}^{s, p}$ .TE $(s)$ or TM $(p)$ component of 2D Fourier transformed electric field transmitted from the substrate $(\bar{\Delta}=S)$ or the cover $(\bar{\Delta}=\mathrm{C})$ to the $w$ th layer

$E_{\bar{\Delta} w, \alpha, \beta}^{s, p}$ $\alpha$ th and $\beta$ th harmonics in $k_{x}$ and $k_{y}$ direction, respectively, of TE (s) or $\mathrm{TM}(p)$ component of 2D Fourier transformed electric field transmitted from the substrate $(\bar{\Delta}=S)$ or the cover $(\bar{\Delta}=\mathrm{C})$ to the $w$ th layer

$\mathbf{E}_{\Delta}^{+}$ ..Fourier transform of the electric field vector radiated by the source $\Delta$ along $\mathbf{z}-(+)$ and $-\mathbf{z}(-)$ directions

$E_{\Delta}^{s, p, \pm}$ TE $(s)$ or TM $(p)$ component of 2D Fourier transformed stationary electric field radiated by the source $\Delta$ along $\mathbf{z}-(+)$ and $-\mathbf{z}(-)$ directions $E_{\Delta, \alpha, \beta}^{s, p, \pm}$ $\alpha$ th and $\beta$ th harmonics in $k_{x}$ and $k_{y}$ direction, respectively, of TE (s) or TM $(p)$ component of 2D Fourier transformed stationary electric field radiated by the source $\Delta$ along $\mathbf{z -}(+)$ and $-\mathbf{z}(-)$ directions

$\mathbf{E}_{\mathrm{T} \bar{\Delta}}$ .Fourier transform of the electric field vector transmitted to the substrate $(\bar{\Delta}=\mathrm{S})$ or the cover $(\bar{\Delta}=\mathrm{C})$

$\overline{\mathbf{E}_{\mathrm{T} \bar{\Delta}}}$ Temporal envelop of the electric field vector transmitted to the substrate $(\bar{\Delta}=\mathrm{S})$ or the cover $(\bar{\Delta}=\mathrm{C})$

$E_{T \bar{\Delta}}^{\chi}$ Amplitude of the 2D FT electric field transmitted from system to the cover $(\bar{\Delta}=\mathrm{C})$ or to the substrate $(\bar{\Delta}=\mathrm{S})$

$E_{T \bar{\Delta}}^{\chi, \Delta}$ Amplitude of the 2D FT electric field transmitted from the source $\Delta$ to the cover $(\bar{\Delta}=\mathrm{C})$ or to the substrate $(\bar{\Delta}=\mathrm{S})$

$\mathbf{E}_{\mathbf{r}}^{\mathrm{FT}}$ .Fourier transform of the reflected electric field vector

$\mathbf{E}_{\mathrm{t}}^{\mathrm{FT}}$ ...Fourier transform of the transmitted electric field vector

$\mathbf{E}_{\mathbf{X}, \mathbf{Z}}^{\mathbf{F F}}$ ..Transmitted electric field at the far field of a dipole oriented along $x$ or $z$-axis

$\mathbf{E}_{\perp, \| l}^{\mathrm{FF}}$ Transmitted electric field at the far field of a dipole perpendicularly or parallel polarized

$E_{\perp}^{s, p}$ ..TE (s) or TM

p) componen transformed electric field vector radiated by a dipole perpendicularly polarized

$E_{\|}^{s, p}$

TE $(s)$ or TM $(p)$ component of 2-dimensional (2D) Fourier transformed electric field vector radiated by a dipole parallel polarized

$f$.................................Frequency

ff .................................Fill factor

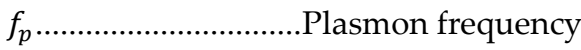


$f_{r}$

F ...Resonance frequency Probability density function describing the probability of a given configuration being occupied

g.............................Decay curve

$g_{\bar{\Delta}}$............................ Decay curve of a system measured at the cover $(\bar{\Delta}=\mathrm{C})$ or to the substrate $(\bar{\Delta}=\mathrm{S})$

Go ...........................Dyadic Greens function in 2D

$h$.............................Polymer layer thickness (ps. in chapter $1, h$ is the Planck constant)

H...............................agnetic field vector

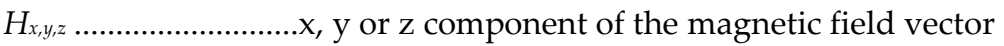

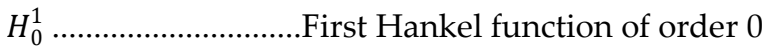

I ...............................Identity matrix

J ……......................... Current density vector

k.............................Wavevector

k.............................Wavevector modulus

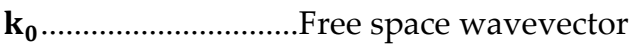

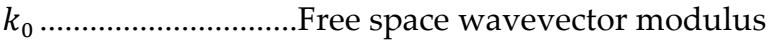

$\mathbf{k}_{\mathbf{i}}$...........................Wavevector inside medium $i$

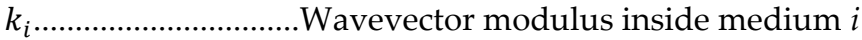

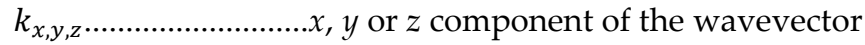

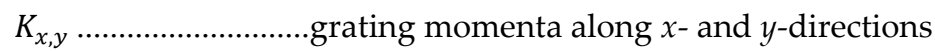

$k_{z i}$ or $k_{z}^{i} \ldots \ldots \ldots \ldots \ldots \ldots . . . . . . . z$ component of the wavevector inside the $i$ th layer

$k_{z \Delta} \ldots \ldots \ldots \ldots \ldots \ldots . . . . . . . . . . . . . . . z$ component of the wavevector inside the layer where the $\mathrm{L}(\Delta=\mathrm{L}), \mathrm{S}$ $(\Delta=S)$ or $C(\Delta=C)$ is located

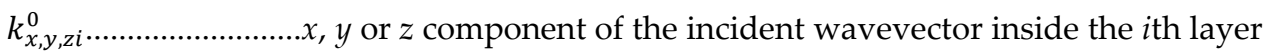

$k_{x, y}^{\alpha, \beta} \ldots \ldots \ldots \ldots \ldots \ldots \ldots \ldots \ldots . . . . . . . . . . . .1 h$ and $\beta$ th harmonics of the $x$ or $y$ component of the wavevector in $k_{x}$ and $k_{y}$ direction, respectively

$k_{z \Delta}^{\alpha, \beta} \ldots \ldots \ldots \ldots \ldots \ldots \ldots \ldots \ldots . . . . . . . . . .1$ th and $\beta$ th harmonics of the $x$ or $y$ component of the wavevector in $k_{x}$ and $k_{y}$ direction, respectively, inside the layer where the source $\mathrm{L}$ $(\Delta=\mathrm{L}), \mathrm{S}(\Delta=\mathrm{S})$ or $\mathrm{C}(\Delta=\mathrm{C})$ is placed

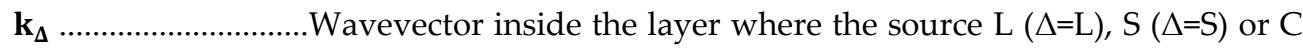
$(\Delta=\mathrm{C})$ is placed

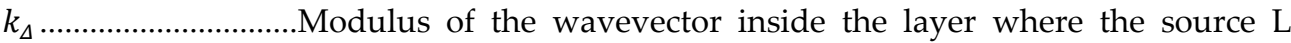
$(\Delta=\mathrm{L}), \mathrm{S}(\Delta=\mathrm{S})$ or $\mathrm{C}(\Delta=\mathrm{C})$ is placed

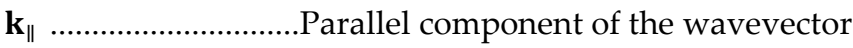


$\mathbf{k}_{\|}^{\alpha, \beta}$

$l$.

$\mathbf{L}_{\mathbf{s}, \mathbf{p}}^{\mathbf{i}}$

$\mathbf{M}$

$\mathbf{M}^{\mathrm{s}, \mathrm{p}}$

$N$

$N_{x, y}$

$n$

p

$\mathrm{p}_{\Delta}$

$p$

$P$

$P_{\text {eff }}$

$p_{x, y, z}$

$P_{x, z}$

$P_{\perp, \|}$

$P_{\text {eff }}^{\perp, \|}$

$P_{x, z}^{2 D}$

$\mathbf{P}_{\mathbf{s}, \mathbf{p}}^{\mathbf{i}}$

$\mathbf{P}_{\mathbf{s}, \mathbf{p}}^{\Delta}$ vector responsible for decomposing the TE

$(s)$ or TM $(p)$ polarized electric field in $x-, y$ and $z$-components inside the layer where the source $L(\Delta=\mathrm{L}), \mathrm{S}(\Delta=\mathrm{S})$ or $\mathrm{C}(\Delta=\mathrm{C})$ is located.

$q$

$Q_{x, z}$ distance between the QE and the HMM

$Q_{\bar{\Delta}}$ Power radiated by a dipole oriented along $x$ - or $z$-axis

$Q_{\bar{\Delta}}$ .Power radiated by a system to the cover $(\bar{\Delta}=\mathrm{C})$ or to the substrate $(\bar{\Delta}=S)$

$Q_{\perp, \|}$ ..Radiated power of a perpendicular $(\perp)$ or parallel $(I I)$ QE

$Q_{x, Z}^{2 D}$

$\mathbf{r}$

$\mathbf{r}_{\Delta}$

$\mathrm{r}$

$R_{s, p}$ Power radiated by a 2D-dipole oriented along $x$ - or $z$-axis Position vector

Position vector of the source $\mathrm{L}(\Delta=\mathrm{L}), \mathrm{S}(\Delta=\mathrm{S})$ or $\mathrm{C}(\Delta=\mathrm{C})$

Modulus of the position vector

.Reflection coefficient for TE (s) or TM (p) polarization 


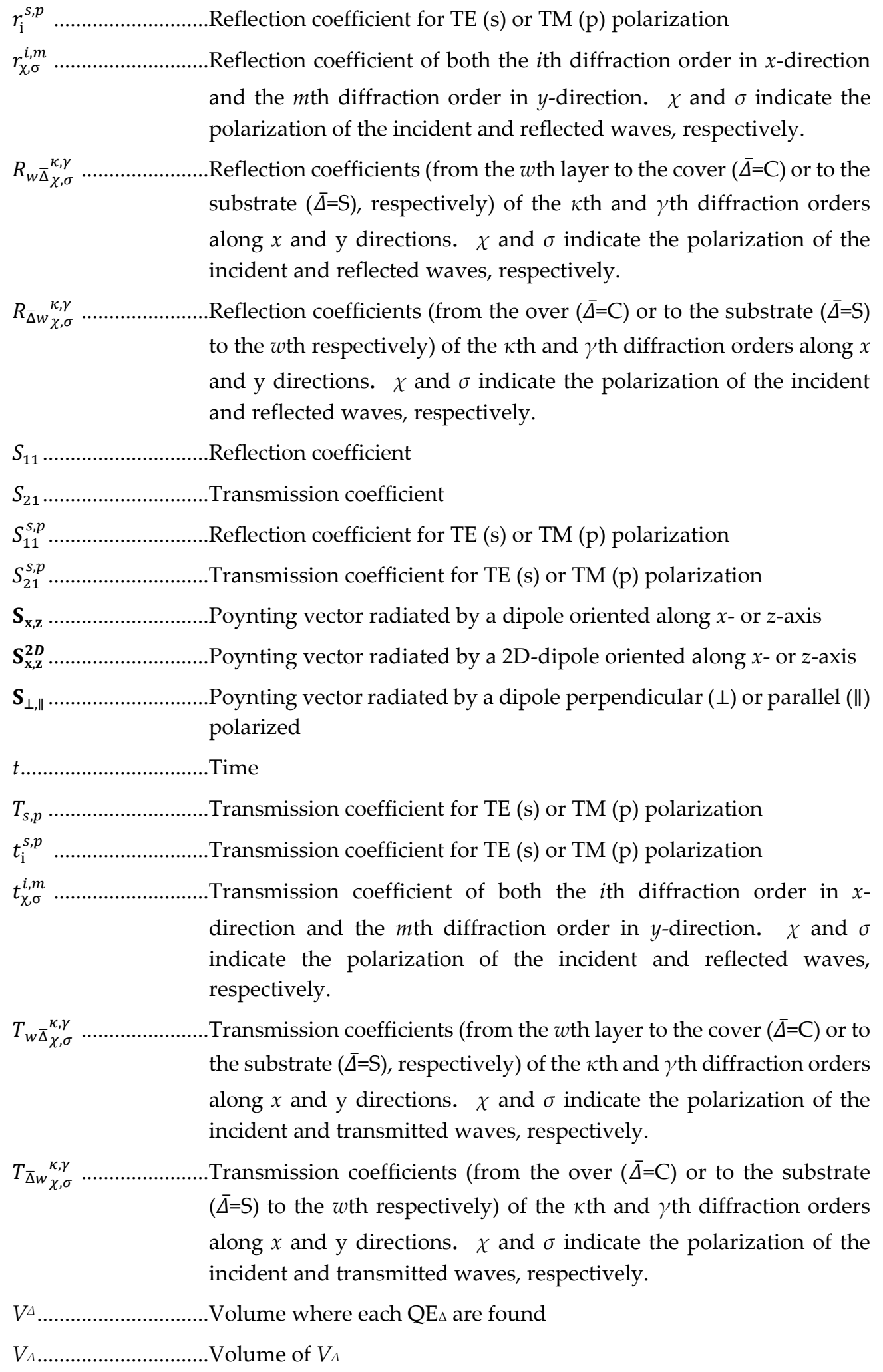




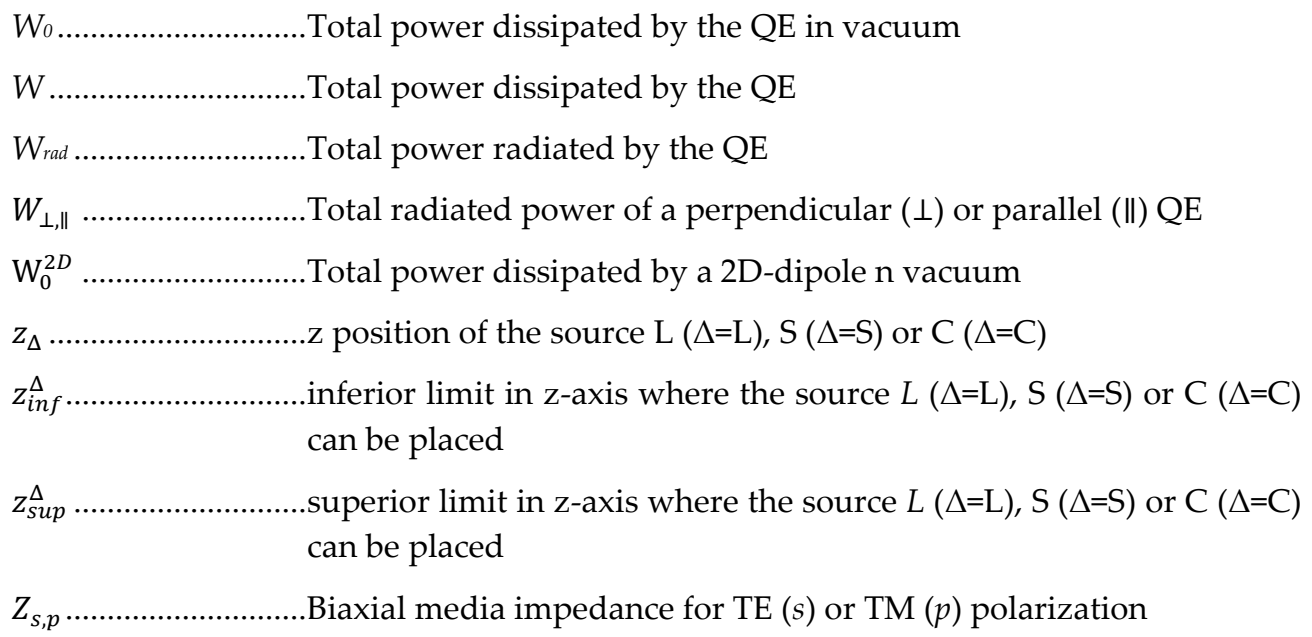

\section{Greek letters}

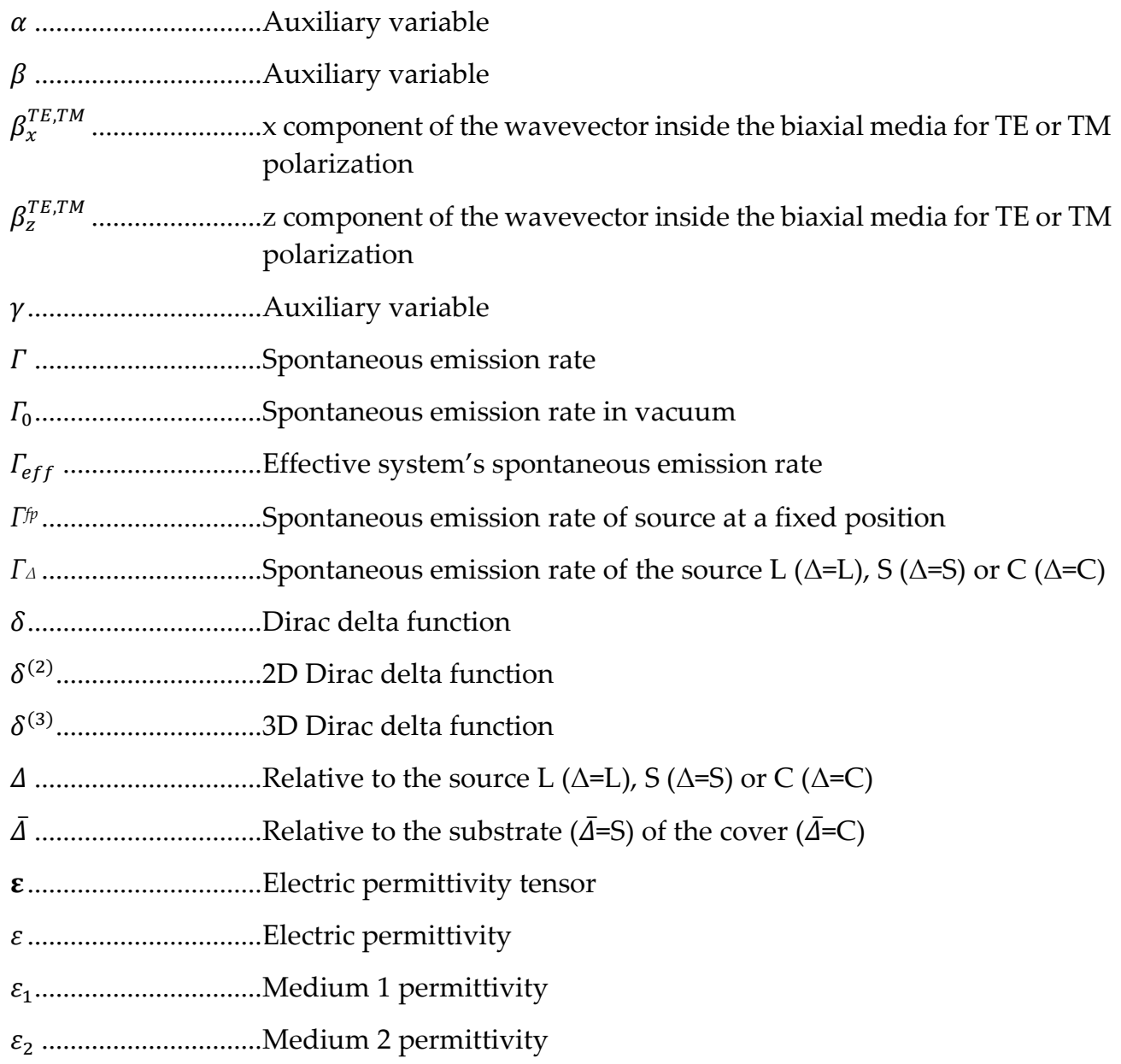




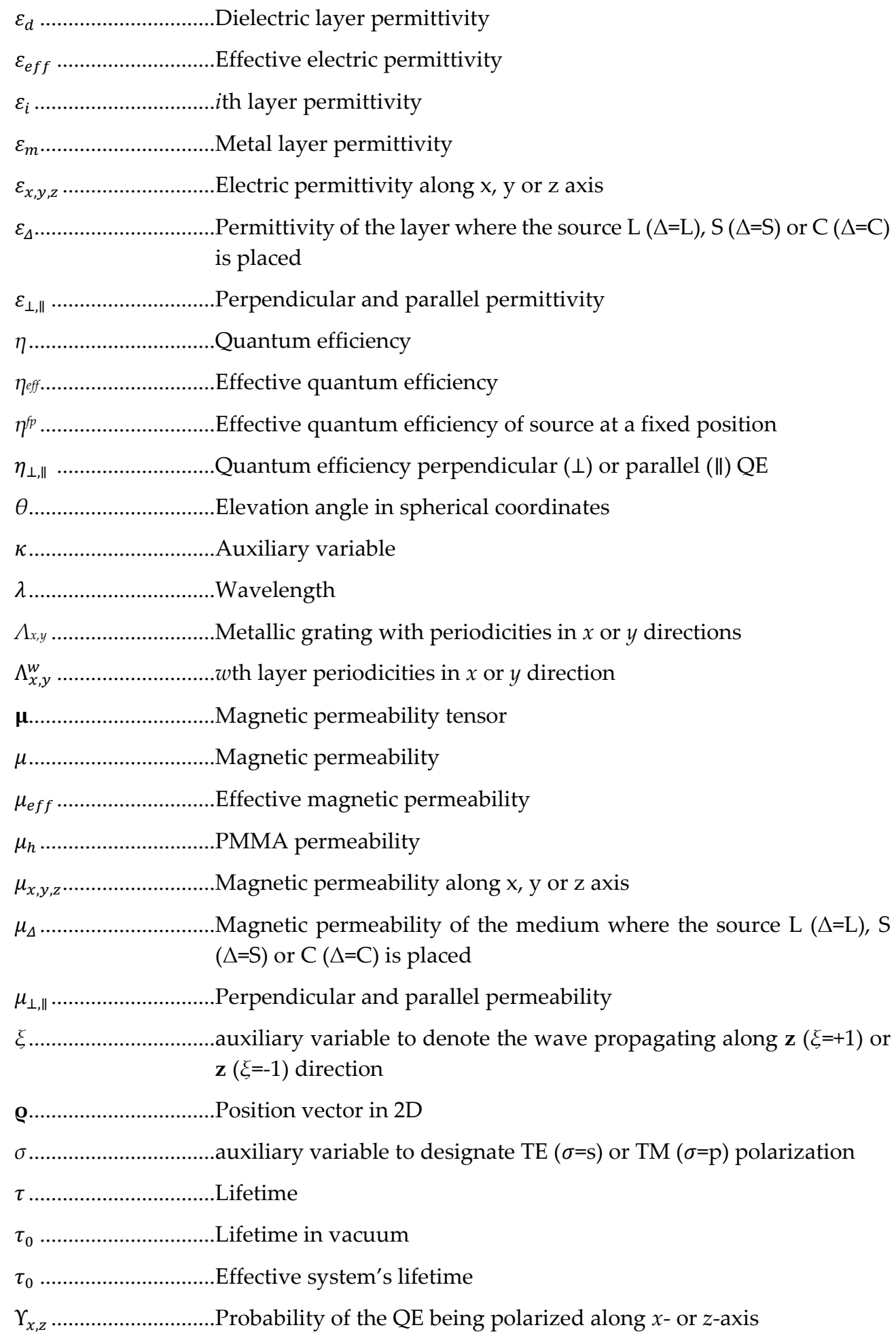




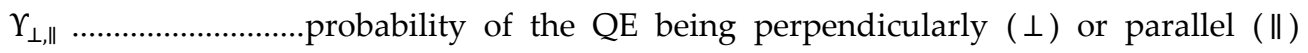
polarized

$\varphi$..............................Azimuthal angle in spherical coordinates

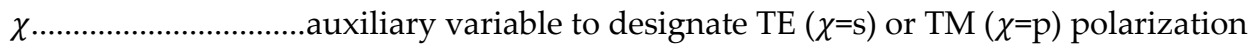

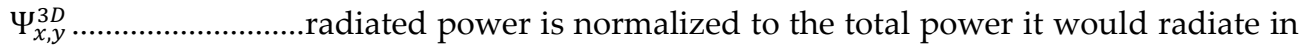
free space of a dipole oriented along $x$ - or $z$-axis

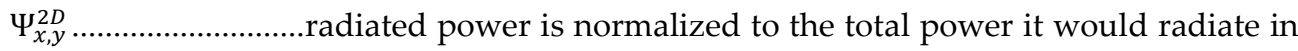
free space of a 2D-dipole oriented along $x$ - or $z$-axis

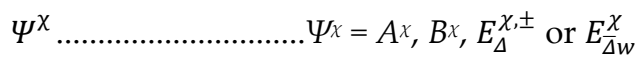

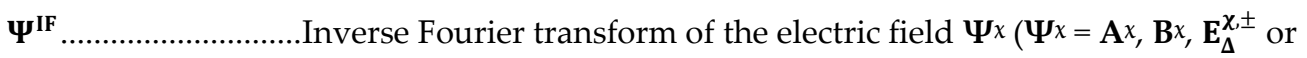
$\left.\mathbf{E}_{\bar{\Delta} \mathbf{w}}^{\chi}\right)$

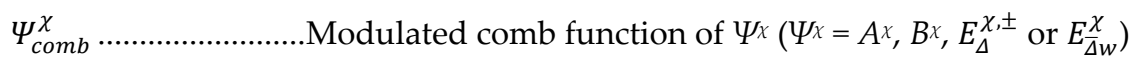

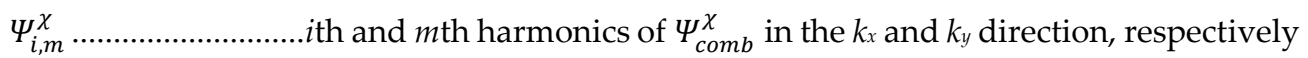

(................................Angular frequency 


\section{List of Figures}

Figure 1.1. (a) Array of elementary cells in a glass (a) and (b) array of macroscopic atoms of a metamaterial (Based on [13]). .............................

Figure 1.2. Metamaterials classification (Adapted from [1])....................................6

Figure 1.3. Isofrequency surfaces of (a) an isotropic dielectric $(\varepsilon \perp=\varepsilon \|)$, (b) a two-sheeted hyperboloid $(\varepsilon \perp>0$ and $\varepsilon \|<0)$, and (c) a one-folded hyperboloid $(\varepsilon \perp<0$ and $\varepsilon \|>0)$. The projection of each isofrequency surface on the plane $\mathrm{k}_{\mathrm{y}} / \mathrm{k}_{0}=0$ is shown at the bottom, where the real and imaginary parts are represented by blue and red lines, respectively. The grey area represents the region where it is possible to have low loss modes inside the stack....................................................8

Figure 1.4. Hyperbolic Metamaterials. (a) metal-dielectric stack and (b) wire medium.

Figure 1.5. Metamaterials homogenization. The metamaterial cell (a) has the same reflection $\left(S_{11}\right)$ and transmission $\left(S_{21}\right)$ coefficients as its homogenised medium (b).

Figure 1.6. Homogenization procedure of a layered medium (a). The layered medium (a) has the same reflection $\left(S_{11}\right)$ and transmission $\left(S_{21}\right)$ parameters as its homogenised medium (b) for each $k_{x}$.

Figure 1.7. Quantum emitters (top) in (a) free space and (b) close to a structure supporting a large number of modes, and their respective energy level diagrams (middle, showing excitation and decay routes) and normalized decay curves (bottom). In (a), a photon with energy $E_{1}=h f_{1}$ excites an electron of the highest occupied molecular orbital ( $\left.\mathrm{S}_{0}\right)$ to the lowest unoccupied molecular orbital $\left(S_{1}\right) .\left(k_{1}, \ldots, k_{N}, k_{n r}, h f_{2}\right)$ and $\left(k_{n r}, h f_{2}\right)$ represent the decay routes for a QE in scenario (a) and (b), respectively. $\left(\Gamma_{0}, \tau_{0}\right)$ and $(\Gamma, \tau)$ are the spontaneous emission rate and the QE lifetime in scenario (a) and (b), respectively

Figure 1.8. (a) Nano-patterned HMM surrounded by QEs [55], [103], [125]-[127].

(b) QEs inside the patterned HMM [128], [129]. (c) Grating on top of the HMM with the QEs embedded in the bottom layers [124]. (d) QEs inside the HMM [57], [120], [121].

Figure 2.1. Metal/dielectric stack with incident wave impinging on the surface from the left. Part of the incident wave is reflected $\left(S_{11}\right)$ and part is transmitted $\left(S_{21}\right)$. The metal and dielectric thicknesses are 1 and $\mathrm{d}$, respectively.

Figure 2.2. Equivalent homogenous anisotropic medium.

Figure 2.3. Diagrams show field components incident on the interface between air and a homogenous, anisotropic medium for (a) TE and (b) TM polarizations 
Figure 2.4. Diagram shows the algorithm for extracting constitutive parameters. 29

Figure 2.5. Diagram illustrating the radiation of a dipole at a distance $d$ from the surface. Note that the magnitude of the reflected wave is polarization dependent (TE or TM).

Figure 2.6. Real (Left column) and imaginary (Right column) parts of the extracted electromagnetic parameters of a layered medium of $\mathrm{Ag} / \mathrm{TiO} 2$ with $\mathrm{f} f=0.1$ for dielectric thickness of 15 (squares), 30 (circles) and $45 \mathrm{~nm}$ (triangles) for [81] (dashed lines, hollow symbols) and the CPR approach (solid lines, full symbols). (a,b) $\varepsilon \perp$, (c,d) $\varepsilon \|$, (e,f) $\mu \perp$ and (g,h) $\mu \|$. The EMT (solid line, stars) results are also plotted for comparison. The inset in (c,d) shows the full magnitude of the real and imaginary parts of $\varepsilon \|$, for the $45 \mathrm{~nm}$ case of [81].......33

Figure 2.7. Real (Left column) and imaginary (Right column) parts of the extracted electromagnetic parameters of a layered medium of $\mathrm{Ag} / \mathrm{TiO} 2$ with $\mathrm{ff}=0.3$ for dielectric thickness of 15 (squares), 30 (circles) and $45 \mathrm{~nm}$ (triangles) for [81] (dashed lines, hollow symbols) and the CPR approach (solid lines, full symbols). (a,b) $\varepsilon \perp,(c, d) \varepsilon \|$, $(\mathrm{e}, \mathrm{f}), \mu \perp$ and $(\mathrm{g}, \mathrm{h}) \mu \|$. The EMT (solid line, stars) results are also plotted for comparison.

Figure 2.8. Real (left column) and imaginary (right column) parts of (a) $\varepsilon \|$ and (b) $\varepsilon \perp<0$ for 3 (squares), 15 (circles) and 27 (triangles) layers for [81] (dashed lines, hollow symbols) and the CPR approach (solid lines, full symbols). The EMT (solid line, stars) results are also plotted for comparison.

Figure 2.9. Real (left column) and imaginary (right column) parts of the dispersion curves of a $\mathrm{Ag} / \mathrm{SiO} 2$ stack with $f f=0.3(\mathrm{a}, \mathrm{b})$ and $f f=0.5$ $(\mathrm{c}, \mathrm{d})$. The dielectric thicknesses (used as a parameter) is $8 \mathrm{~nm}$ (squares), $16 \mathrm{~nm}$ (circles), $24 \mathrm{~nm}$ (triangles) and $32 \mathrm{~nm}$. (diamonds) for [81] (dashed lines, hollow symbols) and the CPR approach (solid lines, full symbols). Results from the EMT (solid line, stars) are also shown for comparison.

Figure 2.10. $H_{y}$ profile for $f f=0.3((\mathrm{a}, \mathrm{d}))$ and for $f f=0.5((\mathrm{e}, \mathrm{h}))$, for $d=16 \mathrm{~nm}$ and different $\left|k_{x} / k_{0}\right|$ values $\left(\left|k_{x} / k_{0}\right|=0.5\right.$ in $(\mathrm{a}, \mathrm{e}),\left|k_{x} / k_{0}\right|=3.5$ in $(\mathrm{b}, \mathrm{f}),\left|k_{x} / k_{0}\right|$ $=6$ in $(\mathrm{c}, \mathrm{g})$ and $\left|k_{x} / k_{0}\right|=8$ in $(\mathrm{d},(\mathrm{h}))$. The solid black line represents the amplitude of $H_{y}$, and the dashed lines represent the boundaries of the layers. The HMM is assumed immersed in air.

Figure 2.11. Dispersion curves for $\mathrm{Ag} / \mathrm{TiO} 2((\mathrm{a})-(\mathrm{d}))$ and $\mathrm{Au} / \mathrm{Si}((\mathrm{e})-(\mathrm{h}))$ stacks with $(\mathrm{a}, \mathrm{e}) \mathrm{ff}=0.2,(\mathrm{~b}, \mathrm{f}), f f=0.3,(\mathrm{c}, \mathrm{g}), f f=0.4$ and $(\mathrm{d}, \mathrm{h}), f f=0.5$. The dielectric thicknesses (used as a parameter) are $8 \mathrm{~nm}$ (squares), $16 \mathrm{~nm}$ (circles), $24 \mathrm{~nm}$ (triangles) and $32 \mathrm{~nm}$ (diamonds). Note that at $f f=0.4$ and $f f=0.5, \mathrm{Ag} / \mathrm{TiO} 2$ corresponds to a single-sheet hyperboloid while $\mathrm{Au} / \mathrm{Si}$ corresponds to a two-sheet hyperboloid surface. 40

Figure 2.12. Purcell factor calculated using (i) the benchmark approach (squares), ii the EMT approach (stars), (iii) [81] (triangles), and (iv) the CPR 
approach (circles). The calculation is carried out for a $\mathrm{Rb}$ atom $10 \mathrm{~nm}$ above the surface of a $\mathrm{Ag} / \mathrm{TiO} 2$ stack with $f f=0.3$ (hollow symbols) and 0.5 (full symbols) for $\lambda=780 \mathrm{~nm}$ (a) and $422 \mathrm{~nm}$ (b).....................42

Figure 3.1. Absorption (red) and emission (blue) of the Cy7...................................48

Figure 3.2. Proposed HMM consisting of 7 alternating layers of silver $\left(\mathrm{Ag}, \varepsilon_{m}=-\right.$ $25+1.71 \mathrm{j})$ and titanium dioxide $\left(\mathrm{TiO}_{2}, \varepsilon_{d}=6.38\right)$. The $\mathrm{Cy} 7$ molecules are embedded in a PMMA film deposited on the top of the HMM. The design wavelength is $773 \mathrm{~nm}$

Figure 3.3. Real (blue) and imaginary (green) parts of the HMM dispersion curve for (a) TE and (b) TM polarizations

Figure 3.4. Proposed structure to model the radiation parameters of QE embedded in a PMMA layer of finite thickness.

Figure 3.5. Normalized $x-(a, c)$ and $z-(b, d)$ electric field components of a dipole perpendicularly $(a, b)$ and parallel $(c, d)$ polarized. The HMM is highlighted in (a-d) with black dashed lines........................................53

Figure 3.7. QE uniformly distributed along the PMMA layer..................................54

Figure 3.6. Normalized dissipated power density $(k 0 \mathrm{~d} W \alpha / \mathrm{d} k x)$ for perpendicular (blue) and parallel (red) polarized QEs for (a) $0 \leq k_{x} / k_{0}$ $\leq 25$ and (b) $0 \leq k_{x} / k_{0} \leq 2$, respectively. The gray area represents the propagation region $\left(k_{x} / k_{0} \leq 1\right)$

Figure 3.8. $\quad$ (a) Purcell factor $\left(P_{\alpha}\right)$ and (b) normalized radiated power $\left(Q_{\alpha} / W_{0}\right)$ of the system proposed in Figure 3.2 calculated as function of the distance between the QE and the HMM surface $(a)$ for perpendicular (blue) and parallel (red) polarizations.

Figure 3.9. Radiation pattern as function of the distance for (a) perpendicular and (b) parallel polarized QEs.

Figure 3.10. Decay behaviour of the quantum emitter considering [ $\Upsilon \perp, \Upsilon \|]$ equals to [1,0] (blue), $[0,1]$ (red) and $[1 / 3,2 / 3]$ (dashed red lines). The gray area represents is the decay region and the QE's decay behavior in free space are shown as dashed light blue lines). To facilitate the $P_{\alpha}$ calculation, we also show $g(t)=1 / \mathrm{e}$ as black dashed line. Note that the $\mathrm{x}$-axis is normalized by $\tau_{0}$.

Figure 3.11. (a) $P_{\text {eff }}$ and (b) $\eta_{\text {eff }}$ as function of $h$ for three scenarios: QEs fully perpendicularly (squares) and parallel (circles) polarized, and QEs equally polarized (triangles). In (a) $P_{\text {eff }}$ is limited to 40 for better visualization, the full curve is shown in the inset.

Figure 4.1. Proposed 3D geometry in which the QEs are modelled as a dipole embedded in medium 1 (with permittivity $\varepsilon_{1}$ ), centered at the origin and at a distance $\mathrm{q}$ from the HMM bottom surface. The grating with periodicities $\Lambda_{x}$ and $\Lambda_{y}$ in $\mathrm{x}$ and y directions, respectively, on top of the HMM is covered with medium 2 (with permittivity $\varepsilon_{2}$ )

Figure 4.2. Proposed 2D geometry in which the QEs are modelled as dipoles embedded in medium 1 (with permittivity $\varepsilon 1$ ), centered at the origin and at a distance $\mathrm{q}$ from the HMM bottom surface. The grating with 
periodicity $\Lambda_{x}$ in $\mathrm{x}$-direction is on top of the HMM and is covered with medium 2 (with permittivity $\varepsilon 2$ ). The HMM and the grating are merged for sake of clarity....

Figure 4.3. Proposed geometry in which the QEs are modelled as a dipole embedded in Medium 1 (with permittivity $\varepsilon_{1}$ ), centered at the origin and at a distance $\mathrm{q}$ from the HMM bottom surface. The gratings with periodicity $\Lambda_{x}$ and $\Lambda_{y}$ in $\mathrm{x}$ and $\mathrm{y}$ directions, respectively, are on top of the HMM and are covered with Medium 2 (with permittivity $\varepsilon_{2}$ )......78

Figure 4.4. (a) Silver Gratings with period $\Lambda_{x}$ and height $a$ on top of a HMM composed of 8 alternating layers of $\mathrm{Ag}(\varepsilon=-25+1.71 \mathrm{j})$ and $\mathrm{TiO}_{2}$ ( $\varepsilon=6.38$ ). (b) Real (squares) and imaginary (circles) parts of the HMM dispersion curve for TM (solid symbols) and TE polarization (hollow symbols).

Figure 4.5. (a) Perpendicularly and (b) parallel polarized normalized emission patterns of a 2D-dipole as function of the grating period. (c) Normalized power radiated by the 2D-dipole $(\Psi \alpha 2 D)$ and $(\mathrm{d})$ Purcell factor, both for a 2D-dipole perpendicularly (red curve) and parallel (blue curve) polarized. The 2D results obtained with a Lumerical FDTD for perpendicularly (squares) and parallel (circles) polarized 2D-dipoles are also shown in (c) and (d) for the sake of comparison. 80

Figure 4.6. Purcell factor (hollow symbols) and normalized radiated power ( $\Psi \alpha 2 D$,full symbols) for perpendicular (square) and parallel (circle) polarization calculated as function of the grating fillfactor (a) and height (b). The Purcell factor is referred to the right axis and the normalized power to the left axis.

Figure 4.7. Normalized squared electric field and emission pattern of a perpendicular (a) and parallel (b) polarized 2D-dipoles.

Figure 4.8. Left and right columns refer to perpendicular and parallel polarized 2D-dipoles, respectively. Purcell factor $(a, b)$ and normalized radiated power $(\mathrm{c}, \mathrm{d})$ calculated as function of the 2D-dipole position. $(\mathrm{a}, \mathrm{c})$ Perpendicular and $(b, d)$ parallel polarization. The colorbar is valid for both figures of each row.

Figure 4.9. 3D-FDTD simulations of the total power radiated to the far field by the perpendicular dipole for $f=30 \%$ (circles), $49 \%$ (squares) and $70 \%$ (triangles).

Figure 4.10. Normalized Poynting vector of a perpendicularly (a) and parallel (d) polarized dipole placed at $\mathrm{q}=10 \mathrm{~nm}$. Normalized electric fields emitted by a perpendicularly $(\mathrm{b}, \mathrm{c})$ and parallel $(\mathrm{e}, \mathrm{f})$ polarized dipole decomposed in $p$ - $(\mathrm{b}, \mathrm{e})$ and $s$-polarization $(\mathrm{c}, \mathrm{f})$.

Figure 4.11. Normalized dissipated power of a perpendicularly (a) and parallel (b) polarized dipole placed at $q=10 \mathrm{~nm}$.

Figure 4.12. Normalized radiated power $(a, c)$ and Purcell factor $(b, d)$ of a perpendicularly $(\mathrm{a}, \mathrm{b})$ and parallel $(\mathrm{c}, \mathrm{d})$ polarized dipole as function of its position variation $\Delta x$ and $\Delta y$ at $q=10 \mathrm{~nm}$. The solid black line in (a-d) represents the periodic array of nano-cylinders edges 
Figure 4.13. Effective Purcell factor $(\mathrm{a}, \mathrm{c}, \mathrm{e})$ and quantum efficiency $(\mathrm{b}, \mathrm{d}, \mathrm{f})$ for HMM_opt (squares) and HMM_ch3 (circles) considering scenarios I $(a, b)$, II $(c, d)$ and III $(e, f)$. $(a, c, e)$ are limited in the $y$-axis. The inset in each plot shows the full curve.

Figure 4.14. (a) and (b) show $\eta$ effm and heff, respectively, as function of Peffm for the scenario III with HMM_opt (red lines) and HMM_ch3 (blue lines).

Figure 5.1. W-layer stack sandwiched between two semi-infinite slabs, i.e., a substrate and a cover layer. The depicted nano-pattern profiles are just an illustration of possible geometries. $\Lambda x w$ and $\Lambda y w$ represent the $w$ th layer unit cell sizes along $\mathrm{x}$ - and $\mathrm{y}$-directions, respectively. The sources S, L and C are embedded within the substrate, wth layer and cover, respectively. The electric fields radiated by the sources $S$ $(\mathbf{E S}+$ and $\mathbf{E S}-)$ and $\mathbf{C}(\mathbf{E C}+$ and $\mathbf{E C}-)$ are transmitted to layer $w$ giving rise to the fields ESw and ECw, respectively. These fields, combined with the electric field radiated by the source L (EL + and EL-), are reflected by layers $w+1$ and $w-1$, creating two stationary fields (A and $\mathbf{B}$ ) inside layer $w$. Moreover, the sum of the electric fields propagating along the $+\mathbf{z}$ and $-\mathbf{z}$ directions inside layer $w$ are transmitted to the cover (ETC) and substrate (ETS), respectively.......97

Figure 5.2. Multilayer stack with $W=11$ built on top of a $\mathrm{SiO}_{2}$ substrate. Layers 1-9 consist of alternating $\mathrm{Ag}$ and $\mathrm{SiO}_{2}$ layers whose thicknesses are $d_{m e}$ and $d_{d i}(15$ and $60 \mathrm{~nm}$ ), respectively, except for layer 9, whose thickness is $d_{m e} / 2$. These layers are patterned with period $\Lambda x s=\Lambda y s$ $=230 \mathrm{~nm}$, fill factor $f 3 D=0.6125(\rho g c=50 \mathrm{~nm})$, filled with polymethylmethacrylate (PMMA). Layer 11 consists of a nanopatterned layer of $\mathrm{TiO}_{2}$ with thickness $d_{c}=50 \mathrm{~nm}$, period $\Lambda x c=\Lambda y c=$ $115 \mathrm{~nm}, f 3 D=0.25(\rho g c=57.5 \mathrm{~nm})$, filled with air (which is also the cover layer). The source QEL is placed inside layer 10 (PMMA) at $a=$ $30 \mathrm{~nm}$ from layer 9 top interface and $b=20 \mathrm{~nm}$ from layer 11 bottom interface. The source QEc is placed in the cover layer at a distance $b_{c}$ from layer 11 top interface.

Figure 5.3. (a) $\Gamma_{L} / \Gamma_{0}$ and $\Gamma_{\mathrm{C}} / \Gamma_{0}$ using 3D-SAM (red and blue solid lines) and FDTD (symbols). This figure also shows $\Gamma_{c} / \Gamma_{0}$ obtained with 3D-SAM when QEL is not present (dashed lines). (b) $Q_{C} / W_{0}$ with (solid line) and without (dashed line) QEL.

Figure 5.4. (a) $\mathrm{HMM}$ consisting of 9 alternating $\mathrm{Ag} / \mathrm{SiO}_{2}$ layers on top of $\mathrm{SiO}_{2}$ substrate. The PMMA cover layer with thickness $h$ is positioned on top of the HMM. The (b) and (c) maps show the values of $h$ and $\eta_{\text {eff, }}$ respectively, as function of the metal $\left(d_{m e}\right)$ and dielectric $\left(d_{d i}\right)$ thicknesses required to achieve $P=10$. The black lines in (b) and (c) separate the regions where the stack behaves as a metal, a HMM and a dielectric, according to [101].

Figure 5.5. (a) 2D-NHMM consisting of 9 alternating $\mathrm{Ag} / \mathrm{SiO}_{2}$ layers with period $\Lambda_{x}$ and fill factor $f 2 D$ on top of a $\mathrm{SiO}_{2}$ substrate. The PMMA host 
layer with thickness $h$ is on top of the NHMM. (b) and (c) are the $\Gamma_{x} / \Gamma_{0}$ and $\eta_{x}$ maps, respectively, as function of $\Lambda_{x}$ and $f 2 D$ of a 2D-dipole placed at the center of the ridge at a distance $a=10 \mathrm{~nm}$ from the NPHM surface. The dashed black lines in (b) and (c) represent the LSPP, while the white lines represent the $f 3 D$ of a 3D structure with $\rho_{g}=$ $50 \mathrm{~nm}$

Figure 5.6. Schematics of the three NPHM analyzed with the 3D-SAM: (a) S1 non-patterned, (e) S2 - patterned along the $x$-axis and (i) S3 patterned along the $x$ - and $y$-axes. $\Gamma_{\alpha} / \Gamma_{0}$ as function of position inside the PMMA layer for structures S1 (b,c,d), S2 (f,g,h) and S3 (j,k,l) assuming $\alpha=z(\mathrm{~b}, \mathrm{f}, \mathrm{j}), x(\mathrm{c}, \mathrm{g}, \mathrm{k})$ and $y(\mathrm{~d}, \mathrm{~h}, \mathrm{l})$.

Figure 5.7. $\quad \eta_{\alpha}$ as function of position inside the host layer on top of structures S1 $(\mathrm{a}, \mathrm{b}, \mathrm{c}), \mathrm{S} 2(\mathrm{~d}, \mathrm{e}, \mathrm{f})$ and S3 $(\mathrm{g}, \mathrm{h}, \mathrm{i})$ assuming $\alpha=z(\mathrm{a}, \mathrm{d}, \mathrm{g}), x(\mathrm{~b}, \mathrm{e}, \mathrm{h})$ and $y$ $(\mathrm{c}, \mathrm{f}, \mathrm{i})$.

Figure 5.8. Calculated $P(\mathrm{a})$ and $\eta_{\text {eff }}(\mathrm{b})$ for structures S1 (blue line), S2 (squares) and S3 (circles) for $10 \mathrm{~nm} \leq h \leq 50 \mathrm{~nm}$.

Figure 6.1. NPHM fabrication steps. (a) shows the alternate deposition of the dielectric/metal layers on the top of a substrate. Then, the FIB is used to create the patterned on the deposited layers (b). Finally, the polymer embedded with the QEs are deposited via spin-coat over the NPHM

Figure 6.2. NPHM for sensing application. Changing the refractive index of the liquid flowing inside the microfluidic channels provoke a change in the NPHM resonant frequency, as shown in the figure inset. Note that the inset graphic is just an illustration about the sensor's behavior, and does not represent any simulated structure.

Figure A.1. Homogenization scheme based on the Drude-Lorentz model. (a) shows the original metamaterial cells to be homogenized while (b) shows the homogenized medium.

Figure A.2. Homogenization scheme of metamaterial cells with the TL model ..129

Figure A.3. Diagram of the unit cell of a three cells thick metamaterial slab. Periodic boundary conditions are used at $y= \pm 2.5 \mathrm{~mm}$ and $\mathrm{z}= \pm 2.5 \mathrm{~mm}$. Dimensions are in $\mathrm{mm}$.

Figure A.4. Scattering parameters for the three cells thick metamaterial slab shown in Figure A.2 (a). The solid symbols represent $S_{11}$ and the hollow symbols $S_{21}$. Scattering parameters for the three cells thick metamaterial slab shown in Fig. 3. The solid symbols represent $S_{11}$ and the hollow symbols $S_{21}$. Squares represent parameters extracted with HFSS (benchmark), circles with the (proposed) TL method, and triangles with the DL method.

Figure A.5. Real (solid symbols) and imaginary (hollow symbols) parts of the permeability (a) and permittivity (b) for a three cells thick 
metamaterial slab obtained with HFSS (squares), DL method (circles), and TL method (triangles).

Figure A.6. Relative error for the DL (squares) and TL (circles) methods. The DL error peaks at the material resonance frequency. The inset highlights the average error of the proposed method.

Figure B.1. Process to clean the MS. 136

Figure B.2. The spin coater and the parameters utilized to deposit the PMMA layers on the top of the MS.

Figure B.3. Thickness (a) and roughness of 10 samples of the solution with PMMA and dichloromethane spin coated on the top of a MS.

Figure B.4. Thickness (a) and roughness of 10 samples of the solution with PMMA and dichloromethane spin coated on the top of a MS.

Figure B.5. Cy7 spin-coated on the top of the MS 138

Figure B.6. Radiation pattern for a perpendicularly (blue) and parallel (red) polarized dipole. The figure also shows the best position to put the sensors to measure the power and lifetime of the Cy7 for each polarization.

Figure B.7. Proposed setup to measure the lifetime of the Cy7 dyes for parallel

(a) and perpendicular (b) polarization.

Figure B.8. Proposed setup to measure the lifetime of the Cy7 dyes for parallel

(a) and perpendicular (b) polarization. 140

Figure B.9. Experimental (dashed line) and theoretical (solid line) emission spectrum of the Cy7. The experimental data was fitted using a Gaussian profile (red dashed line).

Figure B.10. Instrument reference function (IFR, blue), the measured decay (red) and the decay fit to eliminate the noise (black) for parallel (a) and perpendicular (b) polarized QEs.

Figure B.11. Experimental (dashed line) and theoretical (solid line) emission spectrum of the Cy7. The experimental data was fitted using a Gaussian profile (red dashed line).

Figure B.12. (a-d) shows the pictures from the HMM surface taken by the SEM with different zooms. As can be seen, the HMM surface is not smooth and could not be used for lifetime measurements. 



\section{List of Tables}

Table 3.1. System decay parameters $\left(\tau / \tau 0, P_{\text {eff }}\right.$ and $\left.\eta_{\text {eff }}\right)$ for perpendicular, parallel and equally polarized QEs.

Table A.1. Initial Values for the Approximation Method

.132

Table A.2. Homogenization parameters for the three cells thick metamaterial slab shown in Figure A.1 (a). 



\section{Bibliography}

[1] N. Engheta and R. W. Ziolkowski, Metamaterials - Physics and Engenieering Explorations, 1st ed. New Jersey: John Wiley \& Sons, 2006.

[2] T. J. Cui, R. Liu, and D. R. Smith, Metamaterials: Theory, Design and Applicaitons, 1st ed. Boston, MA: Springer US, 2010.

[3] A. K. Sarychev and V. M. Shalaev, Electrodynamics Of Metamaterials, 1st ed. World Scientific, 2007.

[4] S. Zouhdi, A. Sihvola, and A. P. Vinogradov, Metamaterials and Plasmonics: Fundamentals, Modelling, Applications, 1st ed. Springer Science \& Business Media, 2008.

[5] F. Capolino, Theory and Phenomena of Metamaterials, 1st ed. CRC Press, 2009.

[6] J. B. Pendry, D. Schurig, and D. R. Smith, "Controlling electromagnetic fields.," Science, vol. 312, no. 5781, pp. 1780-2, Jun. 2006.

[7] J. J. Yang, M. Huang, and J. Sun, "Double Negative Metamaterial Sensor Based on Microring Resonator," IEEE Sens. J., vol. 11, no. 10, pp. 2254-2259, Oct. 2011.

[8] R. W. Ziolkowski and A. D. Kipple, "Application of double negative materials to increase the power radiated by electrically small antennas," IEEE Trans. Antennas Propag., vol. 51, no. 10, pp. 2626-2640, Oct. 2003.

[9] J. B. Pendry, “Negative Refraction Makes a Perfect Lens," Phys. Rev. Lett., vol. 85, no. 18, pp. 3966-3969, Oct. 2000.

[10] P. Alitalo and S. Tretyakov, "Electromagnetic cloaking with metamaterials," Mater. Today, vol. 12, no. 3, pp. 22-29, Mar. 2009.

[11] U. Leonhardt, “Optical conformal mapping.," Science, vol. 312, no. 5781, pp. 1777-80, Jun. 2006.

[12] D. R. Smith, J. B. Pendry, and M. C. K. Wiltshire, "Metamaterials and negative refractive index.," Science, vol. 305, no. 5685, pp. 788-92, Aug. 2004.

[13] N. I. Zheludev, “A Roadmap for Metamaterials,” Opt. Photonics News, vol. 22, no. 3, p. 30, Mar. 2011.

[14] J. D. Jackson, Classical Electrodynamics, Third Edit. Wiley, 1989.

[15] J. C. Bose, "On the Rotation of Plane of Polarisation of Electric Waves by a Twisted Structure," Proc. R. Soc. London, vol. 63, no. 1, pp. 146-152, 1898.

[16] K. F. Lindman, “Über eine durch ein isotropes System von spiralförmigen Resonatoren erzeugte Rotationspolarisation der elektromagnetischen Wellen," Ann. Phys., vol. 368, no. 23, pp. 621-644, 1920.

[17] W. E. Kock, “Metallic Delay Lenses," Bell Syst. Tech. J., vol. 27, no. 1, pp. 58-82, Jan. 1948.

[18] J. Brown, "Artificial dielectrics having refractive indices less than unity," Proc. IEE Part IV Inst. Monogr., vol. 100, no. 5, pp. 51-62, 1953. 
[19] W. Rotman, "Plasma simulation by artificial dielectrics and parallel-plate media," IRE Trans. Antennas Propag., vol. 10, no. 1, pp. 82-95, Jan. 1962.

[20] G. H. B. Thompson, "Unusual Waveguide Characteristics associated with the Apparent Negative Permeability obtainable in Ferrites," Nature, vol. 175, no. 4469, pp. 1135-1136, Jun. 1955.

[21] V. G. Veselado, "The Electrodynamics of Substances with Simultaneously Negative Values of $\varepsilon$ and $\mu$," Physics-Uspekhi, vol. 10, no. 4, pp. 509-514, 1968.

[22] J. B. Pendry, A. J. Holden, W. J. Stewart, and I. Youngs, “Extremely Low Frequency Plasmons in Metallic Mesostructures," Phys. Rev. Lett., vol. 76, no. 25, pp. 4773-4776, Jun. 1996.

[23] J. B. Pendry, A. J. Holden, D. J. Robbins, and W. J. Stewart, "Magnetism from conductors and enhanced nonlinear phenomena [J]," IEEE Trans. Microw. Theory Tech., vol. 47, no. 11, pp. 2075-2084, 1999.

[24] D. Smith, W. Padilla, D. Vier, S. Nemat-Nasser, and S. Schultz, “Composite Medium with Simultaneously Negative Permeability and Permittivity," Phys. Rev. Lett., vol. 84, no. 18, pp. 4184-4187, May 2000.

[25] R. A. Shelby, D. R. Smith, and S. Schultz, "Experimental verification of a negative index of refraction.," Science, vol. 292, no. 5514, pp. 77-9, Apr. 2001.

[26] Z. N. Chen, "Bandwidth Enhancement of a Single-Feed Circularly Polarized Antenna Using a Metasurface," IEEE Antennas Propag. Mag., pp. 39-46, 2016.

[27] S. Yan, P. J. Soh, and G. A. E. Vandenbosch, “Compact All-Textile Dual-Band Antenna Loaded with Metamaterial-Inspired Structure," IEEE Antennas Wirel. Propag. Lett., vol. 14, pp. 1486-1489, 2015.

[28] M. C. Johnson, S. L. Brunton, N. B. Kundtz, and J. N. Kutz, "Sidelobe canceling for reconfigurable holographic metamaterial antenna," IEEE Trans. Antennas Propag., vol. 63, no. 4, pp. 1881-1886, 2015.

[29] T. Ergin, N. Stenger, P. Brenner, J. B. Pendry, and M. Wegener, "Three-dimensional invisibility cloak at optical wavelengths.," Science, vol. 328, no. 5976, pp. 337-9, Apr. 2010.

[30] S. S. Islam, M. R. I. Faruque, and M. T. Islam, "An Object-Independent ENZ Metamaterial-Based Wideband Electromagnetic Cloak," Sci. Rep., vol. 6, no. March, pp. 2-11, 2016.

[31] K. V. Sreekanth et al., "Hyperbolic metamaterials-based plasmonic biosensor for fluid biopsy with single molecule sensitivity," EPJ Appl. Metamaterials, vol. 4, p. 1, 2017.

[32] K. V. Sreekanth et al., "Extreme sensitivity biosensing platform based on hyperbolic metamaterials," Nat. Mater., vol. 15, no. March, pp. 4-11, 2016.

[33] L. Cong, S. Tan, R. Yahiaoui, F. Yan, W. Zhang, and R. Singh, "Experimental demonstration of ultrasensitive sensing with terahertz metamaterial absorbers: A comparison with the metasurfaces," Appl. Phys. Lett., vol. 106, no. 3, 2015.

[34] L. Markley and G. V. Eleftheriades, "Meta-screens and near-field antenna-arrays: A new perspective on subwavelength focusing and imaging," Metamaterials, vol. 5, no. 2-3, pp. 97-106, Jun. 2011. 
[35] P. A. Belov, Y. Hao, and S. Sudhakaran, "Subwavelength microwave imaging using an array of parallel conducting wires as a lens," Phys. Rev. B, vol. 73, no. 3, p. 033108, Jan. 2006.

[36] K. Feng, G. Harden, D. L. Sivco, and A. J. Hoffman, "Subdiffraction Confinement in All-Semiconductor Hyperbolic Metamaterial Resonators," ACS Photonics, vol. 4, no. 7, pp. 1621-1626, 2017.

[37] C. L. Cortes, W. Newman, S. Molesky, and Z. Jacob, “Quantum nanophotonics using hyperbolic metamaterials," J. Opt., vol. 14, no. 6, p. 063001, 2012.

[38] A. Poddubny, I. Iorsh, P. A. Belov, and Y. Kivshar, "Hyperbolic metamaterials," Nat. Photonics, vol. 7, no. 12, pp. 948-957, 2013.

[39] W. D. Newman, C. L. Cortes, and Z. Jacob, “Enhanced and directional single-photon emission in hyperbolic metamaterials," J. Opt. Soc. Am. B-Optical Phys., vol. 30, no. 4, pp. 766-775, 2013.

[40] A. V. Chebykin, A. A. Orlov, A. S. Shalin, A. N. Poddubny, and P. A. Belov, "Strong Purcell effect in anisotropic $\varepsilon$-near-zero metamaterials," Phys. Rev. B, vol. 91, no. 20, p. 205126, 2015.

[41] A. F. da Mota, A. Martins, P. Bertarini, and B.-H. V. Borges, "Dispersion Management with Microwave Chirped Pulses in Metamaterials in the Negative Refraction Regime," IEEE Antennas Wirel. Propag. Lett., pp. 1-1, 2015.

[42] J. S. Gomez-Diaz, S. Gupta, A. Alvarez-Melcon, and C. Caloz, "Investigation on the Phenomenology of Impulse-Regime Metamaterial Transmission Lines," IEEE Trans. Antennas Propag., vol. 57, no. 12, pp. 4010-4014, Dec. 2009.

[43] E. Lheurette, G. Houzet, J. Carbonell, O. Vanbesien, and D. Lippens, "Omega-Type Balanced Composite Negative Refractive Index Materials," IEEE Trans. Antennas Propag., vol. 56, no. 11, pp. 3462-3469, Nov. 2008.

[44] M. Silveirinha and N. Engheta, "Design of matched zero-index metamaterials using nonmagnetic inclusions in epsilon-near-zero media," Phys. Rev. B, vol. 75, no. 7, p. 075119, Feb. 2007.

[45] B. Edwards, A. Alù, M. Young, M. Silveirinha, and N. Engheta, "Experimental Verification of Epsilon-Near-Zero Metamaterial Coupling and Energy Squeezing Using a Microwave Waveguide," Phys. Rev. Lett., vol. 100, no. 3, p. 033903, Jan. 2008.

[46] V. Caligiuri, M. Palei, M. Imran, L. Manna, and R. Krahne, “Planar Double-EpsilonNear-Zero Cavities for Spontaneous Emission and Purcell Effect Enhancement.," ACS Photonics, p. acsphotonics.8b00121, 2018.

[47] M. G. Silveirinha and N. Engheta, "Theory of supercoupling, squeezing wave energy, and field confinement in narrow channels and tight bends using $\varepsilon$ near-zero metamaterials," Phys. Rev. B - Condens. Matter Mater. Phys., vol. 76, no. 24, pp. 1-17, 2007.

[48] M. Silveirinha and A. Alù, “Overview of theory and applications of epsilon-near-zero materials," URSI Gen. Assem., p. 44, 2008.

[49] D. R. Smith and D. Schurig, "Electromagnetic Wave Propagation in Media with Indefinite Permittivity and Permeability Tensors," Phys. Rev. Lett., vol. 90, no. 7, p. 
077405, Feb. 2003.

[50] A. A. Orlov, S. V. Zhukovsky, I. V. Iorsh, and P. A. Belov, "Controlling light with plasmonic multilayers," Photonics Nanostructures - Fundam. Appl., vol. 12, no. 3, pp. 213-230, 2014.

[51] Z. Jacob, L. V. Alekseyev, and E. Narimanov, "Optical Hyperlens: Far-field imaging beyond the diffraction limit," Opt. Express, vol. 14, no. 18, p. 8247, Sep. 2006.

[52] A. Kaur, S. Banerjee, W. Zhao, J. Venkataraman, and Z. Lu, "Deep Subwavelength Power Concentration-Based Hyperbolic Metamaterials," Int. J. Opt., vol. 2012, pp. 16, 2012.

[53] X. Luo et al., "Nanofocusing of circularly polarized Bessel-type plasmon polaritons with hyperbolic metamaterials," Mater. Horiz., vol. 4, pp. 290-296, 2017.

[54] J. Zhou, A. F. Kaplan, L. Chen, and L. J. Guo, "Experiment and Theory of the Broadband Absorption by a Tapered Hyperbolic Metamaterial Array," ACS Photonics, vol. 1, no. 7, pp. 618-624, 2014.

[55] D. Lu, J. J. Kan, E. E. Fullerton, and Z. Liu, “Enhancing spontaneous emission rates of molecules using nanopatterned multilayer hyperbolic metamaterials.," Nat. Nanotechnol., vol. 9, no. 1, pp. 48-53, 2014.

[56] A. N. Poddubny, P. A. Belov, P. Ginzburg, A. V. Zayats, and Y. S. Kivshar, "Microscopic model of Purcell enhancement in hyperbolic metamaterials," Phys. Rev. B, vol. 86, no. 3, p. 035148, Jul. 2012.

[57] T. Galfsky, H. N. S. Krishnamoorthy, W. Newman, E. E. Narimanov, Z. Jacob, and V. M. Menon, “Active hyperbolic metamaterials: enhanced spontaneous emission and light extraction," Optica, vol. 2, no. 1, p. 62, 2015.

[58] S. V Zhukovsky et al., "Hyperbolic metamaterials based on quantum-dot plasmonresonator nanocomposites," Opt. Express, vol. 22, no. 15, pp. 18290-18298, 2014.

[59] G. T. Papadakis, D. Fleischman, A. Davoyan, P. Yeh, and H. A. Atwater, “Optical magnetism in planar metamaterial heterostructures," Nat. Commun., vol. 9, no. 1, 2018.

[60] a. M. Nicolson and G. F. Ross, "Measurement of the Intrinsic Properties of Materials by Time-Domain Techniques," IEEE Trans. Instrum. Meas., vol. 19, no. 4, pp. 377-382, 1970.

[61] D. R. Smith and S. Schultz, "Determination of effective permittivity and permeability of metamaterials from reflection and transmission coefficients," Phys. Rev. B, vol. 65, no. 19, p. 195104, Apr. 2002.

[62] Z. Li, K. Aydin, and E. Ozbay, "Retrieval of effective parameters for bianisotropic metamaterials with omega shaped metallic inclusions," Photonics Nanostructures Fundam. Appl., vol. 10, no. 3, pp. 329-336, Jun. 2012.

[63] J. J. Barroso and U. C. Hasar, "Constitutive Parameters of a Metamaterial Slab Retrieved by the Phase Unwrapping Method," J. Infrared, Millimeter, Terahertz Waves, vol. 33, no. 2, pp. 237-244, Jan. 2012.

[64] F.-J. Hsieh and W.-C. Wang, "Full extraction methods to retrieve effective refractive index and parameters of a bianisotropic metamaterial based on material dispersion models," J. Appl. Phys., vol. 112, no. 6, p. 064907, 2012. 
[65] U. C. Hasar, J. J. Barroso, T. Karacali, and M. Ertugrul, “Reference-plane-invariant and thickness- and branch-index-independent retrieval of effective parameters of bianisotropic metamaterials," AIP Adv., vol. 5, no. 1, p. 017123, 2015.

[66] H. Chen et al., "Left-handed materials composed of only S-shaped resonators," Phys. Rev. E, vol. 70, no. 5, p. 057605, Nov. 2004.

[67] X. Chen, T. Grzegorczyk, B.-I. Wu, J. Pacheco, and J. Kong, "Robust method to retrieve the constitutive effective parameters of metamaterials," Phys. Rev. E, vol. 70, no. 1, p. 016608, Jul. 2004.

[68] D. R. Smith, D. C. Vier, T. Koschny, and C. M. Soukoulis, "Electromagnetic parameter retrieval from inhomogeneous metamaterials," Phys. Rev. E, vol. 71, no. 3, p. 036617, Mar. 2005.

[69] H. Chen et al., "Magnetic properties of S-Shaped Split-ring resonators," Prog. Electromagn. Res., vol. 51, pp. 231-247, 2005.

[70] L.-X. Ran et al., "Experimental study on several left-handed metamaterials," Prog. Electromagn. Res., vol. 51, pp. 249-279, 2005.

[71] V. V Varadan and R. Ro, “Unique Retrieval of Complex Permittivity and Permeability of Dispersive Materials From Reflection and Transmitted Fields by Enforcing Causality," IEEE Trans. Microw. Theory Tech., vol. 55, no. 10, pp. 2224-2230, Oct. 2007.

[72] U. C. Hasar and C. R. Westgate, "A Broadband and Stable Method for Unique Complex Permittivity Determination of Low-Loss Materials," IEEE Trans. Microw. Theory Tech., vol. 57, no. 2, pp. 471-477, Feb. 2009.

[73] Z. Szabo, G. Park, R. Hedge, and E. Li, “A Unique Extraction of Metamaterial Parameters Based on Kramers-Kronig Relationship," IEEE Trans. Microw. Theory Tech., vol. 58, no. 10, pp. 2646-2653, Oct. 2010.

[74] P. A. Belov et al., "Strong spatial dispersion in wire media in the very large wavelength limit," Phys. Rev. B, vol. 67, no. 11, p. 113103, Mar. 2003.

[75] M. G. Silveirinha, "Additional Boundary Condition for the Wire Medium," IEEE Trans. Antennas Propag., vol. 54, no. 6, pp. 1766-1780, Jun. 2006.

[76] S. I. Maslovski, T. A. Morgado, M. G. Silveirinha, C. S. R. Kaipa, and A. B. Yakovlev, "Generalized additional boundary conditions for wire media," New J. Phys., vol. 12, 2010.

[77] V. M. Agranovich and V. E. Kravtsov, “Notes on crystal optics of superlattices," Solid State Commun., vol. 55, no. 1, pp. 85-90, Jul. 1985.

[78] A. V. Chebykin, A. A. Orlov, C. R. Simovski, Y. S. Kivshar, and P. A. Belov, “Nonlocal effective parameters of multilayered metal-dielectric metamaterials," Phys. Rev. B, vol. 86, no. 11, p. 115420, Sep. 2012.

[79] A. Castanié, J.-F. Mercier, S. Félix, and A. Maurel, “Generalized method for retrieving effective parameters of anisotropic metamaterials," Opt. Express, vol. 22, no. 24, p. 29937, 2014.

[80] X. Zhang and $\mathrm{Y} . \mathrm{Wu}$, "Effective medium theory for anisotropic metamaterials," Sci. Rep., vol. 5, p. 7892, 2015.

[81] G. T. Papadakis, P. Yeh, and H. A. Atwater, "Retrieval of material parameters for 
uniaxial metamaterials," Phys. Rev. B, vol. 91, no. 15, p. 155406, Apr. 2015.

[82] O. Kidwai, S. V. Zhukovsky, and J. E. Sipe, "Effective-medium approach to planar multilayer hyperbolic metamaterials: Strengths and limitations," Phys. Rev. A, vol. 85, no. 5, p. 053842, May 2012.

[83] S. M. Rytov, "Electromagnetic properties of a finely stratified medium," Sov. Phys. JETP, vol. 2, no. 3, pp. 466-475, 1956.

[84] V. Popov, A. V. Lavrinenko, and A. Novitsky, "Operator approach to effective medium theory to overcome a breakdown of Maxwell Garnett approximation," Phys. Rev. B, vol. 94, no. 8, p. 085428, Aug. 2016.

[85] V. Popov, A. V. Lavrinenko, and A. Novitsky, "Surface waves on multilayer hyperbolic metamaterials: Operator approach to effective medium approximation," Phys. Rev. B, vol. 97, no. 12, p. 125428, Mar. 2018.

[86] Ansoft, "High Frequency Structure Simulator." http://www.ansys.com/Products/Simulation+Technology/Electronics/Signal+Integrit y/ANSYS+HFSS.

[87] I. Lumerial Solutions, “FDTD." http://www.lumerical.com/tcad-products/fdtd/.

[88] C. R. Simovski, "Material parameters of metamaterials (a Review)," Opt. Spectrosc., vol. 107, no. 5, pp. 726-753, Dec. 2009.

[89] H. Mosallaei, "FDTD-PLRC technique for modeling of anisotropic-dispersive media and metamaterial devices," IEEE Trans. Electromagn. Compat., vol. 49, pp. 649-660, 2007.

[90] M. Y. Wang et al., "The effect of media parameters on wave propagation in a chiral metamaterials slab using FDTD," Int. J. Numer. Model. Electron. Networks, Devices Fields, vol. 27, no. 1, pp. 109-121, 2014.

[91] G. Lubkowski, R. Schuhmann, and T. Weiland, "Extraction of effective metamaterial parameters by parameter fitting of dispersive models," Microw. Opt. Technol. Lett., vol. 49, no. 2, pp. 285-288, Feb. 2007.

[92] G. Lubkowski, B. Bandlow, R. Schuhmann, and T. Weiland, "Effective modeling of double negative metamaterial macrostructures," IEEE Trans. Microw. Theory Tech., vol. 57, no. 5, pp. 1136-1146, 2009.

[93] A. F. Mota and B.-H. V. Borges, "Improved homogenization procedure based on a Drude-Lorentz dispersive model for metamaterials," Microw. Opt. Technol. Lett., vol. 58, no. 6, pp. 1490-1494, Jun. 2016.

[94] R. Chandrasekar et al., "Lasing Action with Gold Nanorod Hyperbolic Metamaterials," ACS Photonics, vol. 4, no. 3, pp. 674-680, 2017.

[95] B. Fan, D. Filonov, P. Ginzburg, and V. A. Podolskiy, "Low-frequency nonlocal and hyperbolic modes in corrugated wire metamaterials," Opt. Express, vol. 26, no. 13, p. 17541, Jun. 2018.

[96] D. J. Roth et al., "Spontaneous Emission inside a Hyperbolic Metamaterial Waveguide," ACS Photonics, vol. 4, no. 10, pp. 2513-2521, 2017.

[97] Z. Jacob, J.-Y. Kim, G. V. Naik, A. Boltasseva, E. E. Narimanov, and V. M. Shalaev, "Engineering photonic density of states using metamaterials," Appl. Phys. B, vol. 100, 
no. 1, pp. 215-218, Jul. 2010.

[98] J. Kim et al., "Improving the radiative decay rate for dye molecules with hyperbolic metamaterials," Opt. Express, vol. 20, no. 7, p. 8100, Mar. 2012.

[99] Y. Guo, W. Newman, C. L. Cortes, and Z. Jacob, "Applications of Hyperbolic Metamaterial Substrates," Adv. Optoelectron., vol. 2012, no. 1, pp. 1-9, 2012.

[100] L. Ferrari, J. Smalley, Y. Fainman, and Z. Liu, "Hyperbolic metamaterials for dispersion-assisted directional light emission," Nanoscale, pp. 9034-9048, 2017.

[101] A. F. Mota, A. Martins, J. Weiner, F. L. Teixeira, and B.-H. V. Borges, "Constitutive parameter retrieval for uniaxial metamaterials with spatial dispersion," Phys. Rev. B, vol. 94, no. 11, p. 115410, Sep. 2016.

[102] H. I. Lin et al., "Integration of Nanoscale Light Emitters and Hyperbolic Metamaterials: An Efficient Platform for the Enhancement of Random Laser Action," ACS Photonics, vol. 5, no. 3, pp. 718-727, 2018.

[103] K. C. Shen, C. Hsieh, Y. J. Cheng, and D. P. Tsai, “Giant enhancement of emission efficiency and light directivity by using hyperbolic metacavity on deep-ultraviolet AlGaN emitter," Nano Energy, vol. 45, no. January, pp. 353-358, 2018.

[104] K. L. Tsakmakidis, R. W. Boyd, E. Yablonovitch, and X. Zhang, "Large spontaneousemission enhancements in metallic nanostructures: towards LEDs faster than lasers," Opt Express, vol. 24, no. 16, pp. 17916-17927, 2016.

[105] C. L. Degen, F. Reinhard, and P. Cappellaro, “Quantum sensing,” Rev. Mod. Phys., vol. 89, no. 3, pp. 1-39, 2017.

[106] W. Zhang, D. S. Ding, Y. B. Sheng, L. Zhou, B. Sen Shi, and G. C. Guo, "Quantum Secure Direct Communication with Quantum Memory," Phys. Rev. Lett., vol. 118, no. 22, pp. 1-6, 2017.

[107] S.-K. Liao et al., "Satellite-to-ground quantum key distribution," Nature, 2017.

[108] I. E. Zadeh et al., "Deterministic Integration of Single Photon Sources in Silicon Based Photonic Circuits," Nano Lett., vol. 16, no. 4, pp. 2289-2294, Apr. 2016.

[109] L. Novotny and B. Hecht, Principles of Nano-Optics, 2nd ed. Cambridge University Press, 2006.

[110] E. Fermi, Nuclear Physics: A Course Given by Enrico Fermi at the University of Chicago, Revised ed. Chicago: University of Chicago Press, 1974.

[111] E. M. Purcell, "Spontaneous emission probalities at radio frequencies," Phys. Rev., vol. 69 , no. $681,1946$.

[112] M. Pelton, "Modified spontaneous emission in nanophotonic structures," Nat. Photonics, vol. 9, no. 7, pp. 427-435, 2015.

[113] M. Kamandar Dezfouli, R. Gordon, and S. Hughes, "Modal theory of modified spontaneous emission of a quantum emitter in a hybrid plasmonic photonic-crystal cavity system," Phys. Rev. A, vol. 95, no. 1, pp. 1-7, 2017.

[114] N. Michieli, B. Kalinic, C. Scian, T. Cesca, and G. Mattei, “Emission Rate Modification and Quantum Efficiency Enhancement of Er 3+ Emitters by Near-Field Coupling with Nanohole Arrays," ACS Photonics, vol. 5, no. 6, pp. 2189-2199, Jun. 2018. 
[115] M. Sanz-Paz et al., "Enhancing Magnetic Light Emission with All-Dielectric Optical Nanoantennas," Nano Lett., 2018.

[116] T. B. Hoang, G. M. Akselrod, C. Argyropoulos, J. Huang, D. R. Smith, and M. H. Mikkelsen, "Ultrafast spontaneous emission source using plasmonic nanoantennas," Nat. Commun., vol. 6, pp. 1-7, 2015.

[117] I. Suleimanov, G. Molnár, L. Salmon, and A. Bousseksou, "Near-Infrared Luminescence Switching in a Spin-Crossover Polymer Nanocomposite," Eur. J. Inorg. Chem., vol. 2017, no. 28, pp. 3446-3451, Aug. 2017.

[118] Y. Wang, D. W. Malcolm, and D. S. W. Benoit, “Controlled and sustained delivery of siRNA/NPs from hydrogels expedites bone fracture healing," Biomaterials, vol. 139, pp. 127-138, Sep. 2017.

[119] T. Shen et al., "Efficient Tumor Accumulation, Penetration and Tumor Growth Inhibition Achieved by Polymer Therapeutics: The Effect of Polymer Architectures," Biomacromolecules, vol. 18, no. 1, pp. 217-230, Jan. 2017.

[120] K. V. Sreekanth, K. H. Krishna, A. De Luca, and G. Strangi, "Large spontaneous emission rate enhancement in grating coupled hyperbolic metamaterials.," Sci. Rep., vol. 4, p. 6340, 2014.

[121] K. H. K. Rishna, K. V. S. Reekanth, and G. Strangi, “Dye-embedded and nanopatterned hyperbolic metamaterials for spontaneous emission rate enhancement," J. Opt. Soc. Am. B, vol. 33, no. 6, pp. 1038-1043, 2016.

[122] M. A. Noginov et al., "Controlling spontaneous emission with metamaterials.," Opt. Lett., vol. 35, no. 11, pp. 1863-5, Jun. 2010.

[123] T. Galfsky, H. N. S. Krishnamoorthy, W. D. Newman, E. E. Narimanov, Z. Jacob, and V. M. Menon, "Directional out-coupling from active hyperbolic metamaterials," arXiv Prepr. arXiv ..., pp. 1-20, 2014.

[124] A. F. da Mota et al., "Semianalytical Model for Design and Analysis of GratingAssisted Radiation Emission of Quantum Emitters in Hyperbolic Metamaterials," ACS Photonics, vol. 5, no. 5, pp. 1951-1959, May 2018.

[125] X. Yang et al., "Enhancing Communication Bandwidths of Organic Color Converters Using Nanopatterned Hyperbolic Metamaterials," J. Light. Technol., vol. 36, no. 10, pp. 1862-1867, 2018.

[126] L. Li, W. Wang, T. S. Luk, X. Yang, and J. Gao, “Enhanced Quantum Dot Spontaneous Emission with Multilayer Metamaterial Nanostructures," ACS Photonics, vol. 4, no. 3, pp. 501-508, Mar. 2017.

[127] D. Lu et al., "Nanostructuring Multilayer Hyperbolic Metamaterials for Ultrafast and Bright Green InGaN Quantum Wells," Adv. Mater., vol. 30, no. 15, pp. 1-7, 2018.

[128] L. Ferrari, D. Lu, D. Lepage, and Z. Liu, "Enhanced spontaneous emission inside hyperbolic metamaterials," Opt. Express, vol. 22, no. 4, pp. 4301-4306, 2014.

[129] J. S. T. Smalley et al., "Luminescent hyperbolic metasurfaces," Nat. Commun., vol. 8, p. 13793, 2017.

[130] L. Sun and C. Jiang, "Quantum interference in a single anisotropic quantum dot near hyperbolic metamaterials," Opt. Express, vol. 24, no. 7, p. 7719, 2016. 
[131] K. Sainath and F. L. Teixeira, "Robust computation of dipole electromagnetic fields in arbitrarily anisotropic, planar-stratified environments," Phys. Rev. E, vol. 90, no. 063302, 2014.

[132] K. Sainath and F. L. Teixeira, "Tensor Green's function evaluation in arbitrarily anisotropic, layered media using complex-plane Gauss-Laguerre quadrature," Phys. Rev. E, vol. 89, no. 053303, 2014.

[133] M. Born and E. Wolf, Principles of Optics: Electromagnetic Theory of Propagation, Interference and Diffraction of Light, 7th ed. Cambridge University Press, 1999.

[134] a. A. Maradudin and D. L. Mills, "Effect of Spatial Dispersion on the Properties of a Semi-Infinite Dielectric," Phys. Rev. B, vol. 7, no. 6, pp. 2787-2810, Mar. 1973.

[135] G. S. Agarwal, D. N. Pattanayak, and E. Wolf, "Electromagnetic fields in spatially dispersive media," Phys. Rev. B, vol. 10, no. 4, pp. 1447-1475, Aug. 1974.

[136] P. Halevi and R. Fuchs, "Generalised additional boundary condition for non-local dielectrics. I. Reflectivity," J. Phys. C Solid State Phys., vol. 17, no. 21, pp. 3869-3888, Jul. 1984.

[137] P. Halevi, Spatial dispersion in solids and plasmas, 1st ed. North Holland, 1992.

[138] P. Jewsbury, "Electrodynamic boundary conditions at metal interfaces," J. Phys. F Met. Phys., vol. 11, no. 1, pp. 195-206, Jan. 1981.

[139] J. M. McMahon, S. K. Gray, and G. C. Schatz, “Optical Properties of Nanowire Dimers with a Spatially Nonlocal Dielectric Function," Nano Lett., vol. 10, no. 9, pp. 3473-3481, 2010.

[140] K. R. Hiremath, L. Zschiedrich, and F. Schmidt, "Numerical solution of nonlocal hydrodynamic Drude model for arbitrary shaped nano-plasmonic structures using Nédélec finite elements," J. Comput. Phys., vol. 231, no. 17, pp. 5890-5896, 2012.

[141] J. M. McMahon, S. K. Gray, and G. C. Schatz, "Calculating nonlocal optical properties of structures with arbitrary shape," Phys. Rev. B, vol. 82, no. 3, p. 035423, Jul. 2010.

[142] C. Menzel, C. Rockstuhl, T. Paul, F. Lederer, and T. Pertsch, "Retrieving effective parameters for metamaterials at oblique incidence," Phys. Rev. B, vol. 77, no. 19, p. 195328, May 2008.

[143] H. N. S. Krishnamoorthy, Z. Jacob, E. Narimanov, I. Kretzschmar, and V. M. Menon, "Topological transitions in metamaterials.," Science, vol. 336, no. 6078, pp. 205-209, Apr. 2012.

[144] M. Pelton et al., "Efficient Source of Single Photons: A Single Quantum Dot in a Micropost Microcavity," Phys. Rev. Lett., vol. 89, no. 23, p. 233602, Nov. 2002.

[145] S. Hughes, "Enhanced single-photon emission from quantum dots in photonic crystal waveguides and nanocavities," Opt. Lett., vol. 29, no. 22, p. 2659, Nov. 2004.

[146] P. Lodahl et al., "Controlling the dynamics of spontaneous emission from quantum dots by photonic crystals," Nature, vol. 430, no. 7000, pp. 654-657, Aug. 2004.

[147] D. E. Chang, A. S. Sørensen, P. R. Hemmer, and M. D. Lukin, "Quantum Optics with Surface Plasmons," Phys. Rev. Lett., vol. 97, no. 5, p. 053002, Aug. 2006.

[148] Z. Jacob, I. I. Smolyaninov, and E. E. Narimanov, “Broadband Purcell effect: Radiative 
decay engineering with metamaterials," Appl. Phys. Lett., vol. 100, no. 18, pp. 81008116, 2012.

[149] N. M. Litchinitser, A. I. Maimistov, I. R. Gabitov, R. Z. Sagdeev, and V. M. Shalaev, "Metamaterials: electromagnetic enhancement at zero-index transition," Opt. Lett., vol. 33, no. 20, p. 2350, Oct. 2008.

[150] G. W. Ford and W. H. Weber, "Electromagnetic interactions of molecules with metal surfaces," Phys. Rep., vol. 113, no. 4, pp. 195-287, 1984.

[151] Lumerical Inc., "http://www.lumerical.com/tcad-products/fdtd/." .

[152] D. Whittaker and I. Culshaw, "Scattering-matrix treatment of patterned multilayer photonic structures," Phys. Rev. B, vol. 60, no. 4, pp. 2610-2618, 1999.

[153] E. Popov and M. Nevie, "Maxwell equations in Fourier space :," America (NY)., vol. 18, no. 11, pp. 2886-2894, 2001.

[154] M. Liscidini, D. Gerace, L. C. Andreani, and J. E. Sipe, "Scattering-matrix analysis of periodically patterned multilayers with asymmetric unit cells and birefringent media," Phys. Rev. B - Condens. Matter Mater. Phys., vol. 77, no. 3, pp. 1-11, 2008.

[155] R. T. (UFF) Couto, “Green's functions for the wave, Helmholtz and Poisson equations in a two-dimensional boundless domain," Rev. Bras. Ensino Fis., vol. 1304, no. 35, pp. 173-176, 2013.

[156] D. Lu, L. Ferrari, J. J. Kan, E. E. Fullerton, and Z. Liu, “Optimization of Nanopatterned Multilayer Hyperbolic Metamaterials for Spontaneous Light Emission Enhancement," Phys. Status Solidi, vol. 1800263, p. 1800263, 2018.

[157] V. M. Martínez, F. L. Arbeloa, J. B. Prieto, and I. L. Arbeloa, “Characterization of rhodamine 6G aggregates intercalated in solid thin films of laponite clay. 2 fluorescence spectroscopy," J. Phys. Chem. B, vol. 109, no. 15, pp. 7443-7450, 2005.

[158] O. D. Miller, S. G. Johnson, and A. W. Rodriguez, "Effectiveness of thin films in lieu of hyperbolic metamaterials in the near field," Phys. Rev. Lett., vol. 112, no. 15, pp. 1-5, 2014.

[159] T. Li and J. B. Khurgin, "Hyperbolic metamaterials: beyond the effective medium theory," Optica, vol. 3, no. 12, p. 1388, 2016.

[160] S. Axelrod, M. K. Dezfouli, H. M. K. Wong, A. S. Helmy, and S. Hughes, "Hyperbolic metamaterial nanoresonators make poor single-photon sources," Phys. Rev. B, vol. 95, no. 15, pp. 1-7, 2017.

[161] L. Jiang et al., "Multifunctional Hyperbolic Nanogroove Metasurface for Submolecular Detection," Small, vol. 13, no. 30, pp. 1-8, 2017.

[162] K. V. Sreekanth et al., "Enhancing the Angular Sensitivity of Plasmonic Sensors Using Hyperbolic Metamaterials," Adv. Opt. Mater., vol. 4, no. 11, pp. 1767-1772, 2016.

[163] B. Liu et al., "A Plasmonic Sensor Array with Ultrahigh Figures of Merit and Resonance Linewidths down to 3 nm," Adv. Mater., vol. 30, no. 12, pp. 1-7, 2018.

[164] Y. Wang, J. Zhou, and J. Li, “Construction of Plasmonic Nano-Biosensor-Based Devices for Point-of-Care Testing," Small Methods, vol. 1700197, p. 1700197, 2017.

[165] N. Liu et al., "Planar metamaterial analogue of electromagnetically induced transparency for plasmonic sensing," Nano Lett., vol. 10, no. 4, pp. 1103-1107, 2010. 
[166] Y. Shen et al., "Plasmonic gold mushroom arrays with refractive index sensing figures of merit approaching the theoretical limit," Nat. Commun., vol. 4, pp. 1-9, 2013.

[167] C. Cao et al., "Metamaterials-Based Label-Free Nanosensor for Conformation and Affinity Biosensing," ACS Nano, vol. 7, no. 9, pp. 7583-7591, Sep. 2013.

[168] D. K. Roper, W. Ahn, B. Taylor, and A. G. D. Asén, “Enhanced Spectral Sensing by Electromagnetic Coupling With Localized Surface Plasmons on Subwavelength Structures," IEEE Sens. J., vol. 10, no. 3, pp. 531-540, 2010.

[169] D. Zarifi, M. Soleimani, A. Abdolali, and S. E. Hosseininejad, "Parameter reconstruction of materials with off-diagonal anisotropy using the state transition matrix method," AEU - Int. J. Electron. Commun., vol. 68, no. 9, pp. 877-882, 2014. 


\title{
Annex 1 - Physical Review Copyrights permission
}

\author{
APS American Physical Society \\ physics Reuse and Permissions License
}

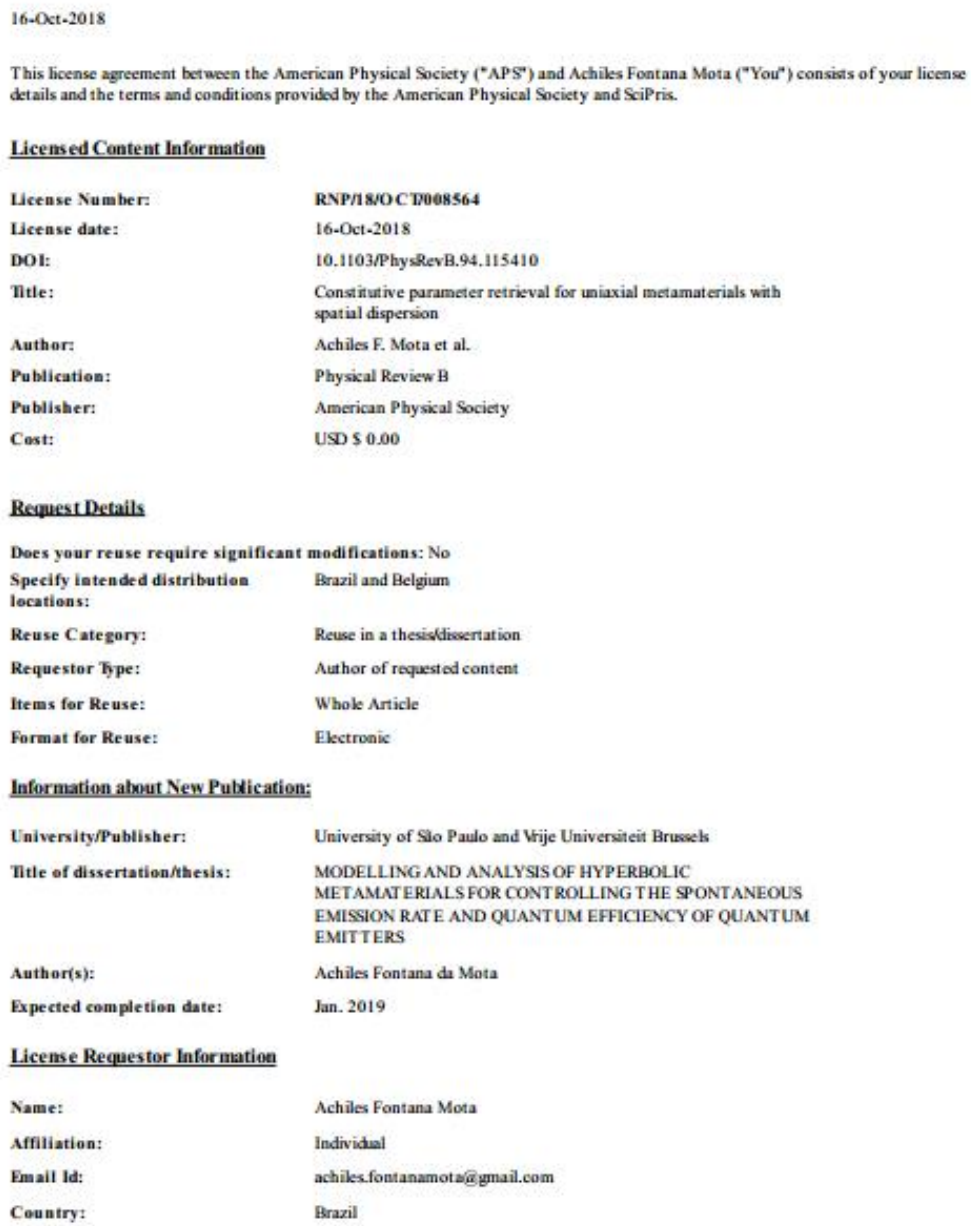

Licensed Cantent Information

$\begin{array}{ll}\text { License Number: } & \text { RNP/180CDees564 } \\ \text { License date: } & 16-0 c t-2018 \\ \text { DOI: } & 10.1103 / \text { hys RevB.94.115410 } \\ \text { Title: } & \begin{array}{l}\text { Constitutive parameter retrieval for uniaxial metamaterials with } \\ \text { spatial dispersion }\end{array} \\ \text { Auther: } & \text { Achiles F. Mota et al. } \\ \text { Publication: } & \text { Physical Review B } \\ \text { Publisher: } & \text { American Physical Society } \\ \text { Cost: } & \text { USD } 50.00\end{array}$

RequestDetails

Does your reuse require significant modifications: No Specify intended distribution Brazil and Belgium lecations:

Reuse Categary:

Reuse in a thesisldiwertation

Requestor Type:

Author of request ed content

Items for Reuse:

Whole Article

Format for Reuse:

Ekectronic

Information about New Publication:

University/Publisher

Title of dissertation/hesis:

Auther(s):

Expected completion date:

License Requestor Information

Name:

Affiliation:

Email ld:

Country:

University of Slo Paulo and Wrije Universiteit Brusesk

MODELLING AND ANALYSISOF HYPERBOLIC

METAMATERIALS FOR CONTROLING THE SPONTANEOUS

EMISSION RATE AND QUANTUM EFFICIENCY OF QUANTUM EMITTERS

Achiles Fontana da Mota

Jan. 2019

Achiles Fontana Mota

Individual

achiles.fontanamotaig gmail.com

Brazil 


\section{APS American Physical Society physics Reuse and Permissions License}

\section{TERMS AND CONDITONS}

The American Physical Society (APS) is pleased to grant the Requestor of this license a non-exclusive, non-transferable permission, limited to Electronic format, provided all criteria outlined below are followed.

1. You must also obxain permission from at least one of the lead authors for each separate work, if you haven't done so already. The author's name and affiliation can be found on the first page of the published Article.

2. For electronic format permissions, Requestor agrees to provide a hyperlink from the reprinted APS material using the source material's DOI on the web page where the work appears. The hyperlink should use the standard DOI resolution URL., http//dix doi.org/(DOI). The hyperlink may be embedided in the copyright credit line.

3. For print format permissions, Requestor agrees to print the required copyright credit line on the first page where the material appears: "Reprinted (abstract/excerpt/figure) with permission from [(FUL.L. REFERENCE CIT NTION) as follows Author's Names, APS Journal Title, Wlume Number, Page Number and Year of Publication.] Copyright (YEAR) by the American Physical Society."

4. Permission granted in this license is for a one-time use and does not include permission for any future editions, updates, databuses, formats or other matters. Permission must be sought for any additional use.

5. Use of the material does not and must not imply any endorsement by APS

6. APS does not imply, purport or intend to grant permission to reuse materials to which it does not hold copyright. It is the requestor's sole responsibility to ensare the licensed material is original to APS and des not contain the copyright of another entity, and that the copyright notice of the figare, photograph, cover or table does not indicate it was reprinted by APS with permission from another source.

7. The permission granted herein is personal to the Requstor for the use specified and is not transferable or assignable without express written permission of APS This license may not be amended except in writing by APS.

8. You may not alter, edit or modify the material in any manner.

9. You may translate the materiak only when translation rights have been granted.

10. APS is not responsible for any errors or omissions due to transhation.

11. You may not use the material for promotional, sales, advertising or marketing purposes.

12. The foregoing license shall not take effect unless and until APS or its agent, Aptara, receives payment in full in accordance with Aptara Billing and Payment Terms and Conditions, which are incorporated herein by reference.

13. Should the terms of this license be violated at any time, APS or Aptara may revoke the license with no refund to you and seek relief to the fullest extent of the laws of the USA. Official written notice will be made using the contact information provided with the permission request. Failure to receive such notice will not nullify revocation of the permission.

14. APS reserves all rights not specifically granted herein.

15. This document, including the Aptara Belling and Payment Terms and Conditions, shall be the entire agreement between the parties relating to the subject matter hereof. 



\section{Annex 2 - ACS Photonics Copyrights permission}

$16 / 10 / 2018$

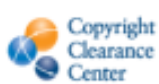

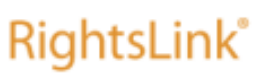

ACS Publications Title:

Rightslink by Copyright Clearance Center

\section{Home

Semianalytical Model for Design and Analysis of Grating-Assisted Radiation Emission of Quantur Emitters in Hyperbolic

Metamaterials

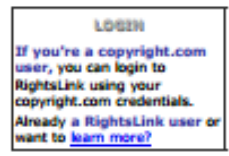

Authorn Achiles F. da Mota, Augusto

ACS Photonics

Publication: ACS Photonics

Dater May 1, 2018

Copyright e 2018, American Chemical Society

PERMISSION/LICENSE IS GRANTED FOR YOUR ORDER AT NO CHARGE

This type of permission/license, instead of the standard Terms \& Conditions, is sent to you because no fee is being charged for your order. Please note the following:

- Permission is granted for your request in both print and electronic formats, and translations.

- If figures and/or tables were requested, they may be adapted or used in part.

- Please print this page for your records and send a copy of it to your publisher/graduate school.

- Appropriate credit for the requested material should be given as follows: "Reprinted (adapted) with permission from (COMPLETE REFERENCE CITATION). Copyright (YEAR) American Chemical Society." Insert appropriate information in place of the capitalized words.

- One-time permission is granted only for the use specified in your request. No additional uses are granted (such as derivative works or other editions). For any other uses, please submit a new request. 



\title{
Annex 3 - MOTL Copyrights permission
}

$1610 / 2018$

RightsLink Printable Lioense

\begin{abstract}
JOHN WILEY AND SONS LICENSE
\end{abstract}
TERMS AND CONDITIONS

Oct 16,2018

This Agreement between Mr. Achiles F. da Mota ("You") and John Wiley and Sons ("John Wiley and Sons") consists of your license details and the terms and conditions provided by John Wiley and Sons and Copyright Clearance Center.

\begin{tabular}{|c|c|}
\hline License Number & 4450960009773 \\
\hline License date & Oct 16,2018 \\
\hline Licensed Content Publisher & John Wiley and Sons \\
\hline Licensed Content Publication & Microwave and Optical Technology Letters \\
\hline Licensed Content Titk & $\begin{array}{l}\text { Improved homogenization procedure based on a Drude-Lorentz } \\
\text { dispersive model for metamaterials }\end{array}$ \\
\hline Leensed Content Author & A. F. Mota, B.H. V. Borges \\
\hline Licensed Content Date & Mar 28, 2016 \\
\hline Licensed Content Volume & 58 \\
\hline Licensed Content Issue & 6 \\
\hline Licensed Content Pages & 5 \\
\hline Type of use & Dissertation/Thesis \\
\hline Requestor type & Author of this wiley article \\
\hline Format & Print and electronic \\
\hline Portion & Full article \\
\hline Will you be transiating? & No \\
\hline $\begin{array}{l}\text { Title of your thesis / } \\
\text { dissertation }\end{array}$ & $\begin{array}{l}\text { MOOELLING AND ANALYSIS OF HYPERBOLIC METAMATERLALS FOR } \\
\text { CONTROLING THE SPONTANEOUS EMISSION RATE AND QUANTUM } \\
\text { EFFICIENCY OF QUANTUM EMITTERS }\end{array}$ \\
\hline Expected completion date & $\operatorname{Jan} 2019$ \\
\hline $\begin{array}{l}\text { Expected size (number of } \\
\text { pages) }\end{array}$ & 140 \\
\hline Requestor Location & $\begin{array}{l}\text { Mr. Achiles F. da Mota } \\
\text { Rua Sete de Setembro, 1458, ap. S01 } \\
\text { Centro }\end{array}$ \\
\hline & $\begin{array}{l}\text { Săo Carlos, São Paulo 13560-180 } \\
\text { Brazil } \\
\text { Attn: Mr. Achiles F. da Mata }\end{array}$ \\
\hline Publisher Tax ID & EU826007151 \\
\hline Total & 0.00 USD \\
\hline
\end{tabular}

Terms and Conditions

\section{TERMS AND CONDITIONS}

This copyrighted material is owned by or exclusively licensed to John Wiley \& Sons, Inc. or one of its group companies (each a"Wiley Company") or handled on behalf of a society with which a Wiley Company has exclusive publishing rights in relation to a particular work (collectively "WILEY"). By clicking "accept" in connection with completing this licensing transaction, you agree that the following terms and conditions apply to this transaction (along with the billing and payment terms and conditions established by the Copyright Clearance Center Inc., ("CCCs Billing and Payment terms and conditions"), at the time that you opened your RightsLink account (these are available at any time at herps/myaccount.cogyright.com).

\section{Terms and Conditions}

- The materials you have requested permission to reproduce or reuse (the "Wiley Materials") are protected by copyright.

- You are hereby granted a personal, non-exclusive, non-sub licensable (on a standalone basis), non-transferable, worldwide, limited license to neprodece the Wiley. Materials for the purposespecified in the licensing process. This license, and any CONTENT (PDF or image file) purchased as part of your order, is for a one-time 
use only and limited to any maximum distribution number specified in the license. The first instance of republication or reuse granted by this license must be completed within two years of the date of the grant of this license (although copies prepared before the end date may be distributed thereafter). The Wiley Materials shall not be used in any other manner or for any other purpose, beyond what is granted in the license. Permission is granted subject to an appropriate acknowledgement given to the author, title of the materia/book/journal and the publisher. You shall also duplicate the copyright notice that appears in the Wiley publication in your use of the Wiley Material. Permission is also granted on the understanding that nowbere in the teat is a previously published source acknowledged for all or part of this Wiley Material. Any third party content is expressly excluded from this permission.

- With respect to the Wiley Materials, all rights are reserved. Except as expressly granted by the terms of the license, no part of the Wiley Materials may be copied, modified, adapted (except for minor reformatting required by the new Publication), translated, reproduced, transferred or distributed, in any form or by any means, and no derivative works may be made based on the Wiley Materials without the prior permission of the respective copyright owner.For STM Signatory Publishers clearing permission under the terms of the STM Permissions Gaidelines only, the terms of the license are extended to include subsequent editions and for editions in other languages, provided such editions are for the work as a whole in situ and does not involve the separate exploitation of the permitted figures or extracts. You may not alter, remove or suppress in any manner any copyright, trademark or other notices displayed by the Wiley Materials. You may not license, rent, sell, loan, lease, pledge, offer as security, transfer or assign the Wiley Materials on a stand-alone basis, or any of the rights granted to you hereunder to any other person.

- The Wiley Materials and all of the intellectual property rights therein shall at all times remain the exclusive property of John Wiley \& Sons Inc, the Wiley Companies, or their respective licensors, and your interest therein is only that of having possession of and the right to reproduce the Wiley Materials pursuant to Section 2 berein during the continuance of this Agreement. You agree that you own no right, title or interest in or to the Wiley Materials or any of the intellectual property rights therein. You shall have no rights hereunder other than the license as provided for above in Section 2. No right, license or interest to any trademark, trade name, service mark or other branding ("Marks") of WILEY or its licensors is granted hereunder, and you agree that you shall not assert any such right, license or interest with respect thereto

- NEITHER WILEY NOR ITS LICENSORS MAKES ANY WARRANTY OR REPRESENTATION OF ANY KIND TO YOU OR ANY THIRD PARTY, EXPRESS, IMPLIED OR STATUTORY, WITH RESPECT TO THE MATERIALS OR THE ACCURACY OF ANY INFORMATION CONTAINED IN THE MATERIALS, INCLUDING, WITHOUT LIMITATION, ANY IMPLIED WARRANTY OF MERCHANTABILITY, ACCURACY, SATISFACTORY QUALITY, FITNESS FOR A PARTICULAR PURPOSE, USABILITY, INTEGRATION OR NON-INFRINGEMENT AND ALL SUCH WARRANTIES ARE HEREBY EXCLUDED BY WILEY AND ITS LICENSORS AND WAIVED BY YOU.

- WILEY shall have the right to terminate this Agreement immediately upon breach of this Agreement by you.

- You shall indemnify, defend and hold harmless WILEY, its Licensors and their respective directors, officers, agents and employees, from and against any actual or threatened claims, demands, causes of action or proceedings arising from any breach of this Agreement by you.

- in No EVENT SHALl WILEY OR ITS LICENSORS BE LIABLE TO YOU OR ANY OTHER PARTY OR ANY OTHER PERSON OR ENTITY FOR ANY SPECIAL, CONSEQUENTIAL, INCIDENTAL, INDIRECT, EXEMPLARY OR PUNITIVE DAMAGES, HOWEVER CAUSED, ARISING OUT OF OR IN CONNECTION WITH THE DOWNLOADING, PROVISIONING, VIEWING OR USE OF THE MATERLALS REGARDLESS OF THE FORM OF ACTION, WHETHER FOR BREACH OF CONTRACT, BREACH OF WARRANTY, TORT, NEGLIGENCE, INFRINGEMENT OR OTHERWISE (INCLUDING, WITHOUT LIMITATION, DAMAGES BASED ON LOSS OF PROFITS, DATA, FILES, USE, BUSINESS OPPORTUNITY OR CLAIMS OF THIRD PARTIES), AND WHETHER OR NOT THE PARTY HAS BEEN ADVISED OF THE POSSIBILITY OF SUCH 
DAMAGES. THIS LIMITATION SHALL APPLY NOTWITHSTANDING ANY FAILURE OF ESSENTIAL PURPOSE OF ANY LIMITED REMEDY PROVIDED HEREIN

- Should any provision of this Agreement be beld by a court of competent jurisdiction to be illegal, invalid, or unenforceable, that provision shall be deemed amended to achieve as nearly as possible the same economic effect as the original provision, and the legality, validity and enforceability of the remaining provisions of this Agreement shall not be affected or impaired thereby.

- The failure of either party to enforce any term or condition of this Agreement shall not constitute a waiver of either party's right to enforce each and every term and condition of this Agreement. No breach under this agreement shall be deemed waived or excused by either party unless such waiver or consent is in writing signed by the party granting such waiver or consent. The waiver by or consent of a party to a breach of any provision of this Agreement shall not operate or be construed as a waiver of or consent to any other or subsequent breach by such other party.

- This Agreement may not be assigned (including by operation of law or otherwise) by you without WILEY's prior written consent.

- Any fee required for this permission shall be non-refundable after thirty (30) days from receipt by the $\mathrm{CCC}$.

- These terms and conditions together with CCC's Billing and Payment terms and conditions (which are incorporated herein) form the entire agreement between you and WILEY concerning this licensing transaction and (in the absence of fraud) supersedes all prior agreements and representations of the parties, oral or written. This Agreement may not be amended except in writing signed by both parties. This Agreement shall be binding upon and inure to the benefit of the parties' successors, legal representatives, and authorized assigns.

- In the event of any conflict between your obligations established by these terms and conditions and those established by CCC's Billing and Payment terms and conditions, these terms and conditions shall prevail.

- WILEY expressly reserves all rights not specifically granted in the combination of (i) the license details provided by you and accepted in the course of this licensing transaction, (ii) these terms and conditions and (iii) $\mathrm{CCC}$ 's Billing and Payment terms and conditions.

- This Agreement will be void if the Type of Use, Format, Circulation, or Requestor Type was misrepresented during the licensing process.

- This Agreement shall be governed by and construed in accordance with the laws of the State of New York, USA, without regards to such state's conflict of law rules. Any legal action, suit or proceeding arising out of or relating to these Terms and Conditions or the breach thereof shall be instituted in a court of competent jurisdiction in New York County in the State of New York in the United States of America and each party hereby consents and submits to the personal jurisdiction of such court, waives any objection to venue in such court and consents to service of process by registered of certified mail, retum receipt requested, at the last known address of such party.

\section{WILEY OPEN ACCESS TERMS AND CONDITIONS}

Wiley Publishes Open Access Articles in fully Open Access Journals and in Subscription journals offering Online Open. Although most of the fully Open Access journals publish open access articles under the terms of the Creative Commons Attribution (CC BY) License only, the subscription journals and a few of the Open Access Journals offer a choice of Creative Commons Licenses. The license type is clearly identified on the article.

The Creative Commons Attribution License

The Creative Commons Attribution License (CC-BY) allows users to copy, distribute and transmit an article, adapt the article and make commercial use of the article. The CC-BY license permits commercial and non-

Creative Commons Attribution Non-Commercial License

The Creative Commons Attribution Non-Commercial(CC-BY-NC)License permits use, distribution and reproduction in any medium, provided the original work is properly cited 


\section{$16 / 10 / 2018$}

and is not used for commercial purposes.(see below)

Creative Commons Attribution-Non-Commercial-NoDerivs License

The Creative Commons Atribution Non-Commercial-NoDerivs License (CC-BY-NC-ND)

permits use, distribution and reproduction in any medium, provided the original work is

properly cited, is not used for commercial purposes and no modifications or adaptations are made. (see below)

Use by commercial "for-profit" organizations

Use of Wiley Open Access articles for commercial, promotional, or marketing purposes

requires further explicit permission from Wiley and will be subject to a fee.

Further details can be found on Wiley Online Library

http//olabout. wiley.com/WileyCDA/Section/id-410895.html

Other Terms and Conditions:

v1.10 Last updated September 2015

Questions? customercare (1) oopyright_com or +1-855-239-3415 (toll free in the US) or $+1-978-646-2777$. 


\section{Layman Summary}

In the past few years, intensive research has been focused on the study of new approaches to control photon emissions of quantum emitters (QEs), specially for telecommunication applications. Controlling the QE's photon emission is important because the faster its photons are emitted, the greater is the number of times it returns to the excited state per second. According to Fermi's Golden rule, this process can be accelerated by creating new decay 'routes' or 'channels' for the QE. One of the most promising approaches of creating decay channels involves a new class of artificial materials, known as hyperbolic metamaterials (HMM). This class of metamaterials allows the propagation of modes with wavevectors (known as high- $k$ modes) much higher than the free-space wavevector. Thus, when a QE is placed sufficiently close to an HMM, its evanescent waves (also with high- $k$ ) are more easily coupled with the HMM high- $k$ modes, consequently reducing the time required for it to decay emitting photons, known as the QE's lifetime. Therefore, shorter lifetimes imply faster modulation speeds (or transmission rates).

One of the key aspects of artificial materials, such as HMM, is the knowledge of their effective electromagnetic properties, achieved via parameter extraction techniques. Particularly for HMM, the high- $k$ modes make the parameter extraction (also known as homogenization procedure) a challenging task. In this sense, we propose in this thesis a new constitutive parameter retrieval approach in which all electromagnetic properties of the medium are obtained taking the high- $k$ modes into account. Moreover, we demonstrate that if this feature is not properly considered in the homogenization procedure, the QE's lifetime calculation is overestimated, therefore impacting its use in telecommunication applications. However, the approach proposed here allows an accurate calculation of the QE's lifetime.

As mentioned, the additional decay channels created by the HMM's high- $k$ modes can be used to decrease the QE's lifetime. However, since most of the power dissipated by the QE is coupled into the HMM as high- $k$ modes (which cannot propagate in free-space), it is thermally dissipated inside the HMM due to these modes high ohmic losses. Consequently, the portion of the power converted into photons is low, resulting in low quantum efficiency. To overcome this issue, some authors have resorted to nanopatterned HMMs (NPHM) to convert high- $k$ modes into photons, aiming at increasing the QE's quantum efficiency. However, much of the NPHMs designs still rely on computationally costly three-dimensional numerical simulations. In this context, we also propose in this thesis a new semi-analytical method capable of optimizing the geometrical parameters of any nano-patterned structure, including NPHMs. In addition to helping increase the QE quantum efficiency while decreasing its lifetime, the proposed method is also capable of reducing the computational burden demanded by the optimization procedure in approximately $96 \%$ when compared to that of numerical methods such as finite-difference in time-domain (FDTD). In summary, this doctoral thesis contributes to pave the way for the development of new high-speed and efficient light sources for telecommunication applications. 